\title{
AFFINE REGION TRACKING AND AUGMENTATION USING MSER AND ADAPTIVE SIFT MODEL GENERATION
}

\author{
A Thesis \\ presented to \\ the Faculty of California Polytechnic State University, \\ San Luis Obispo
}

\author{
In Partial Fulfillment \\ of the Requirements for the Degree \\ Master of Science in Electrical Engineering \\ By \\ Matthew James Marano \\ June 2009
}


(C) 2009

Matthew James Marano

ALL RIGHTS RESERVED 


\section{COMMITTEE MEMBERSHIP}

TITLE:

AFFINE REGION TRACKING AND AUGMENTATION USING MSER AND ADAPTIVE SIFT MODEL GENERATION

AUTHOR:

Matthew James Marano

DATE SUBMITTED:

June 2009

COMMITTEE CHAIR: Lynne Slivovsky

COMMITTEE MEMBER: John A. Saghri

COMMITTEE MEMBER: Jane Zhang 


\section{ABSTRACT \\ AFFINE REGION TRACKING AND AUGMENTATION USING MSER AND ADAPTIVE SIFT MODEL GENERATION}

Matthew James Marano

Relatively complex Augmented Reality (AR) algorithms are becoming widely available due to advancements in affordable mobile computer hardware. To take advantage of this a new method is developed for tracking 2D regions without a prior knowledge of an environment and without developing a computationally expensive world model. In the method of this paper, affinely invariant planar regions in a scene are found using the Maximally Stable Extremal Region (MSER) detector. A region is selected by the user to define a search space, and then the Scale Invariant Feature Transform (SIFT) is used to detect affine invariant keypoints in the region. If three or more keypoint matches across frames are found, the affine transform $A$ of a region is calculated. A 2D image is then transformed by $A$ causing it to appear stationary on the $2 \mathrm{D}$ region being tracked. The search region is tracked by transforming the previous search region by $A$, defining a new location, size, and shape for the search region. Testing reveals that the method is robust to tracking planar surfaces despite affine changes in the geometry of a scene. Many real world surfaces provide adequate texture for successful augmentation of a scene. Regions found multiple frames are consistent with one another, with a mean cross-correlation of 0.608 relating augmented regions. The system can handle up to a $45^{\circ}$ out of plane viewpoint change with respect to the camera. Although rotational changes appear to skew the affine transform slightly, translational and scale based have little distortion and provide convincing augmentations of graphics onto the real world. 


\begin{abstract}
ACKNOWLEDGMENTS
Student Fee Committee for Computer Engineering

Assistant Professor Lynne Slivovsky for Advising and Guidance

Associate Professor John A. Saghri for being a Committee Member

Assistant Professor Jane Zhang for being a Committee Member
\end{abstract}




\section{Table of Contents}

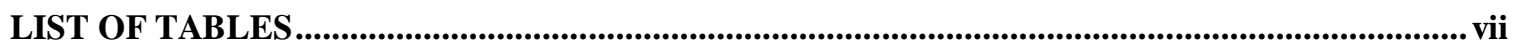

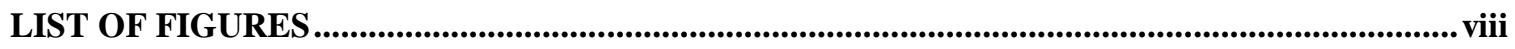

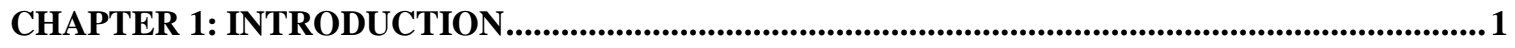

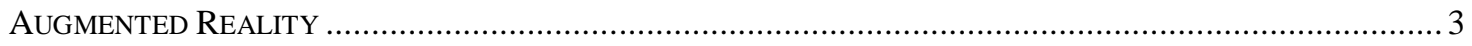

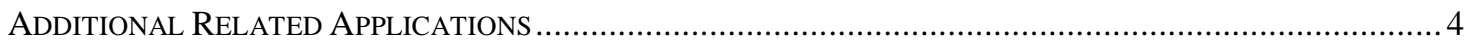

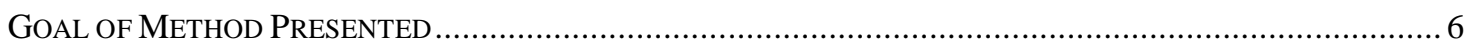

CHAPTER 2: SURVEY OF RELATED WORKS.............................................................................. 7

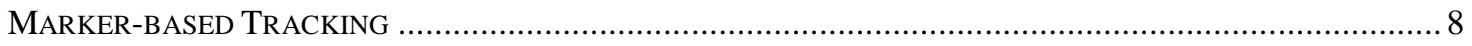

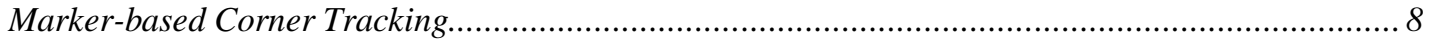

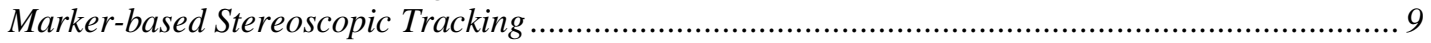

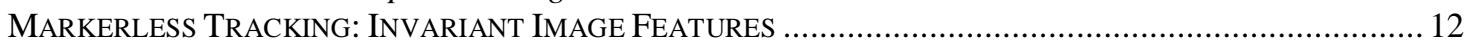

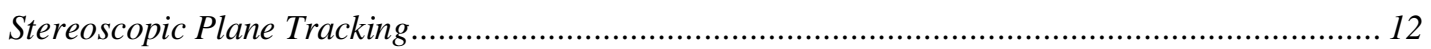

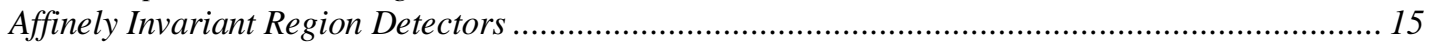

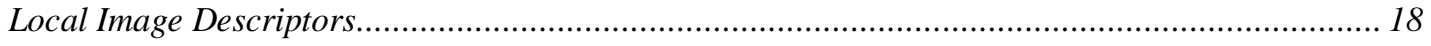

CHAPTER 3: THEORETICAL DERIVATIONS .............................................................................. 21

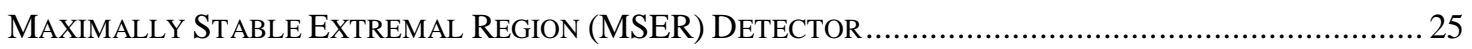

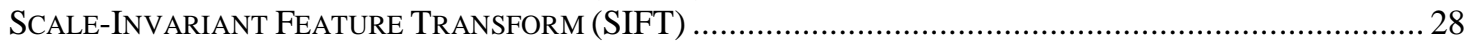

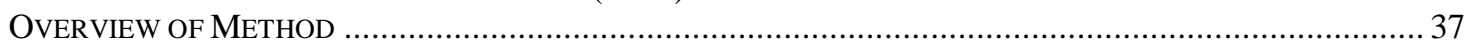

CHAPTER 4: EXPERIMENTAL SETUP AND RESULTS OF TESTING.......................................... 46

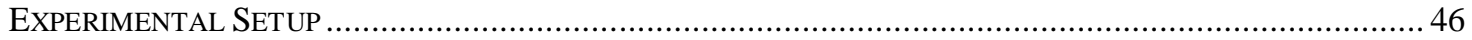

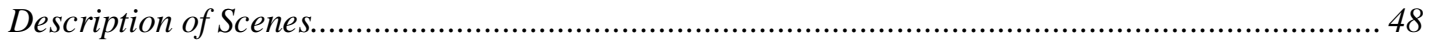

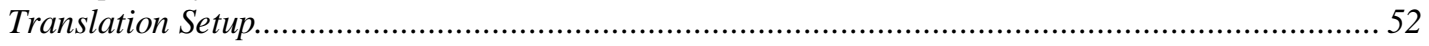

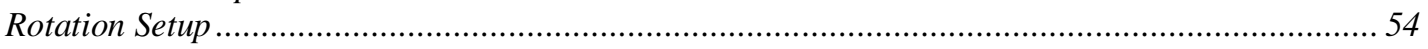

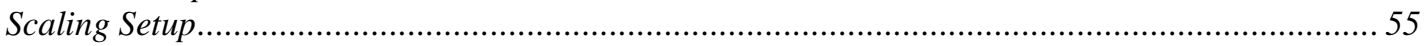

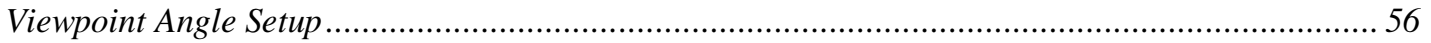

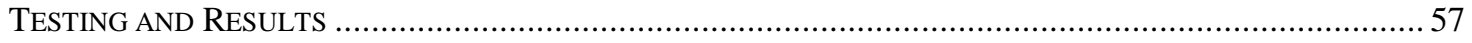

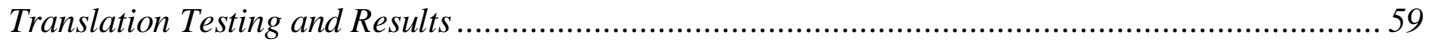

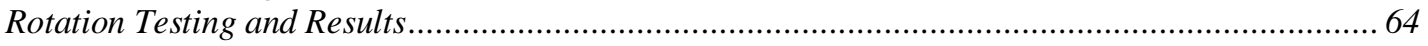

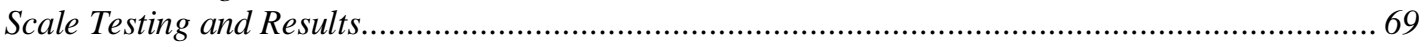

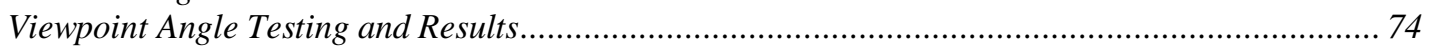

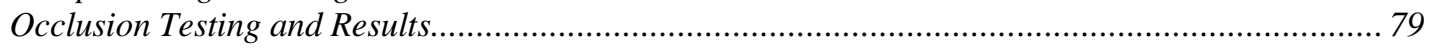

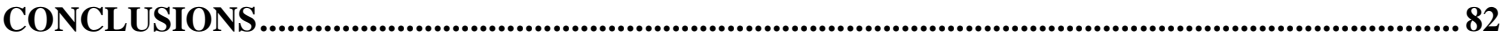

APPENDIX: IMAGES AND DATA PLOTS FOR ADDITIONAL TESTS ......................................... 84

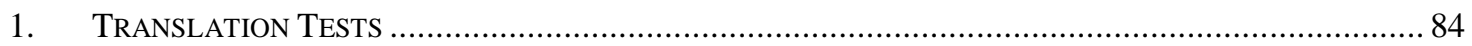

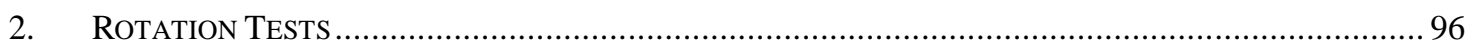

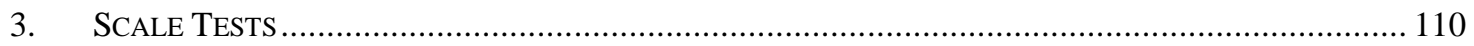

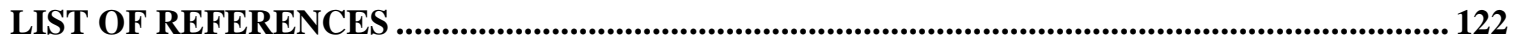




\section{List of Tables}

Table 1: Comparison of Marker-based Tracking and Markerless Tracking......................7

Table 2: Comparison of MSER and SIFT Region Detectors.....................................22

Table 3: Symbols and their Meaning ...................................................................24

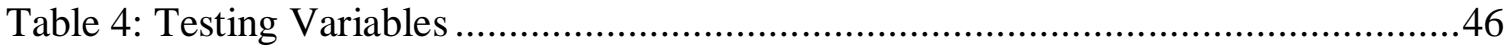

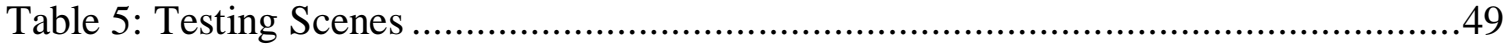

Table 6: Translation Test Geometric Description ...................................................52

Table 7: Rotation Test Geometric Description ..........................................................54

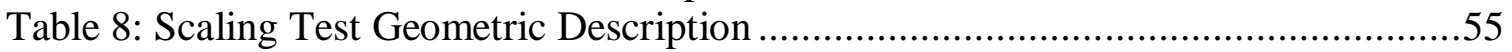

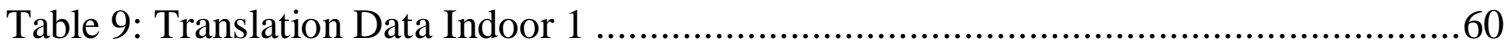

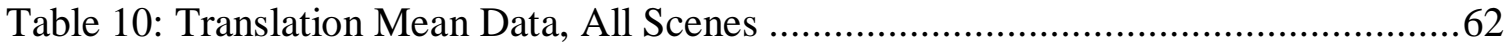

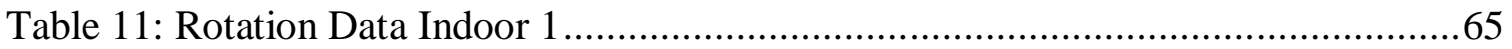

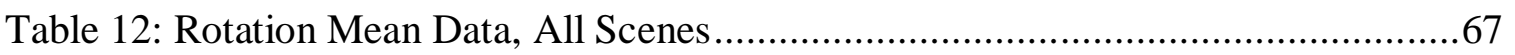

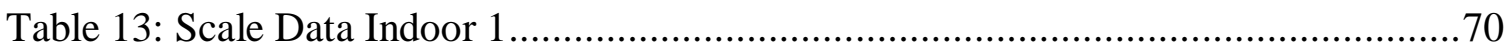

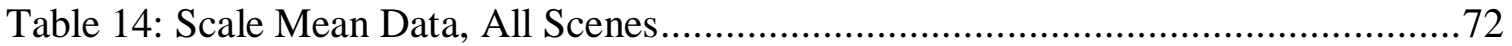

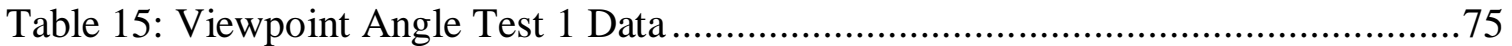

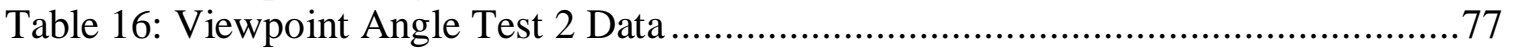

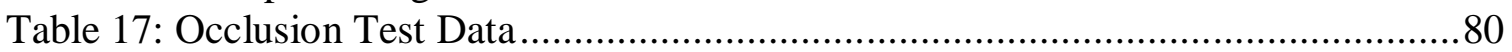

Table 18: Mean Data, All Tests, All Scenes ............................................................ 83

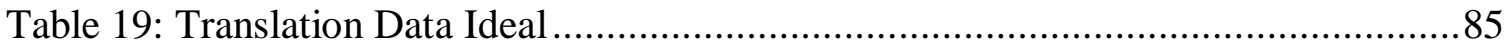

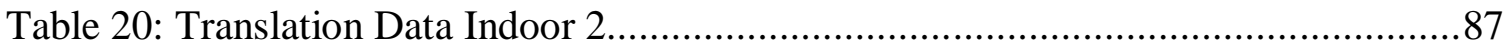

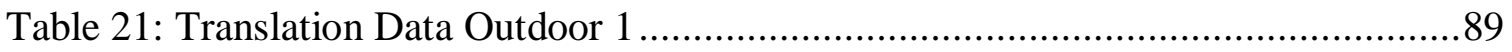

Table 22: Translation Data Outdoor 2 .................................................................. 91

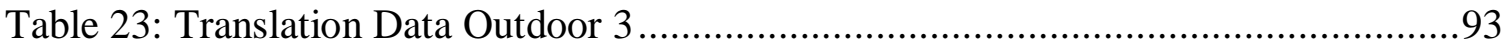

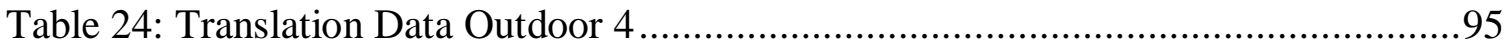

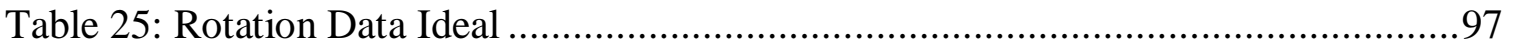

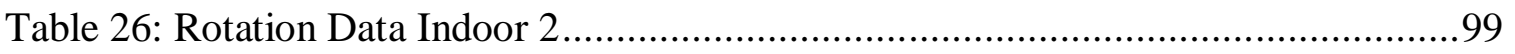

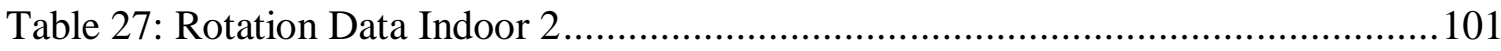

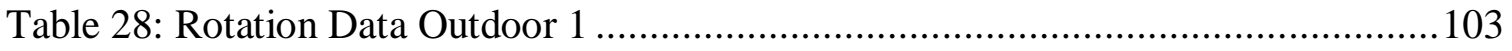

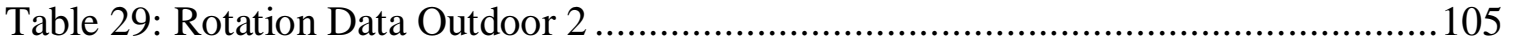

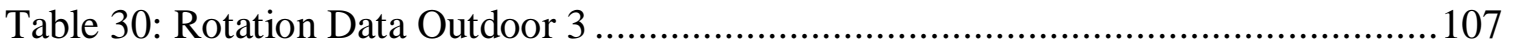

Table 31: Rotation Data Outdoor 4 ...................................................................... 109

Table 32: Scale Data Ideal ................................................................................. 111

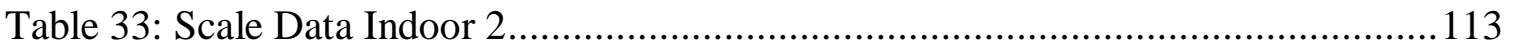

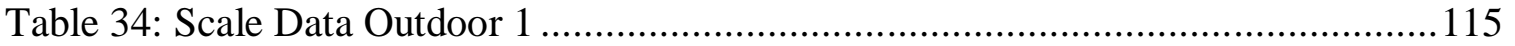

Table 35: Scale Data Outdoor 2 .......................................................................... 117

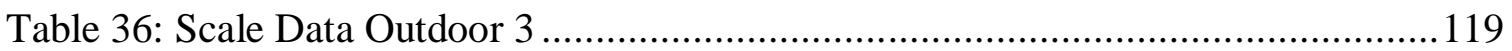

Table 37: Scale Data Outdoor 4 .................................................................... 121 


\section{List of Figures}

Figure 1: Picture of "Wikitude" Application running on Google Android OS [13] ..........2

Figure 2: Head Mounted Display (HMD) intended for Stereoscopic AR [14] .................4

Figure 3: A football players appears to reach for the "yellow line", yet only viewers at home can see it (C SporTVision) ......................................................................5

Figure 4: Corner-based marker tracking Successful Augmentation onto a partially

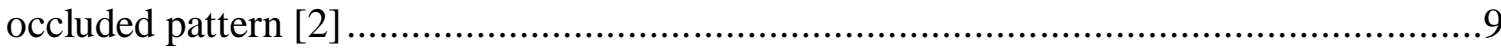

Figure 5: Relationship between the world and camera coordinates. [3] .......................11

Figure 6: Relationship between the marker and camera coordinates. [3]......................11

Figure 7: Fitted line with RANSAC, outliers (red) have no influence on the result.........13

Figure 8: a) the point cloud generated by a stereo vision camera, in which there is no texture on the walls themselves, b) the edges detected in $2 \mathrm{D}, \mathrm{c}$ ) the walls without

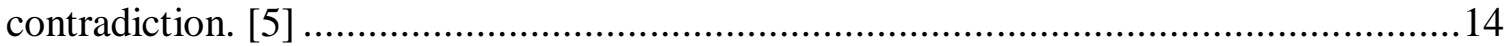

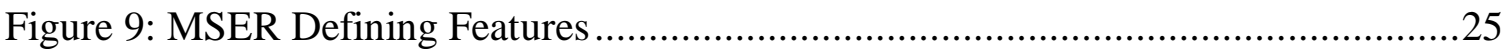

Figure 10: Extremal Regions at Different Thresholds, a) Two minima appear at a low thresholding, b) Minima grow as another region appears at a higher thresholding, c) regions 1 and 3 merge and region 4 appears, d) regions 5 and 6 appear, regions 4 and

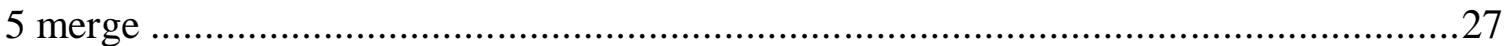

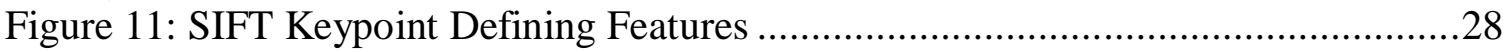

Figure 12: 1-Octave of Difference of Gaussian (DoG) Images ..................................30

Figure 13: Search for Maxima and Minima by Comparison of Scale Space Neighbors...31

Figure 14: SIFT Keypoints at Various Stages of Filtering a) Scale space extrema are detected by the SIFT algorithm, b) Keypoints of low contrast are filtered out, c) Keypoints that are poorly localized are filtered out resulting in final set of Keypoint

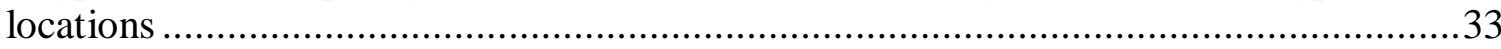

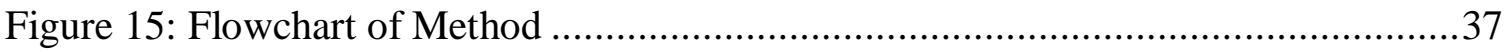

Figure 16: a) Potential Model View Frame, b) Example of Model Frame with Possible Model Ellipses Displayed .38

Figure 17: a) Binary Image of Possible Model Ellipse, b) Possible Model Ellipse and Enclosed SIFT keypoints

Figure 18: SIFT keypoints in Model Frame and Frame 23 of Example, a) Model

View SIFT keypoints, b) Keypoints found in search space of Frame 23, c) Keypoints from model view matched with frame 23, d) keypoints in frame 23 matched with model view ....

Figure 19: Current Ellipse $\mathrm{E}_{23}$ of Frame 23 with closest center point to predicted ellipse.

Figure 20: Images Transformed by $\mathrm{A}_{\mathrm{i}}$, a) Transformed Graphic used for Augmentation, b) Transformed Search Space used to search for keypoints and ellipses in the next frame, c) Augmented version of Example Frame 23. .44

Figure 21: Progression of SIFT keypoints added and removed from model view, a) Original Model View, b) Model View Frame 7, c) Model View Frame 15, d) Model View Frame 23

Figure 22: Images of Real World Scenes (6 total), a) Indoor 1, b) Indoor 2, c)

Outdoor 1, d) Outdoor 2, e) Outdoor 3, f) Outdoor 4. .48 


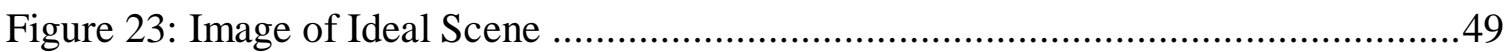

Figure 24: Translation Test Conceptual Diagram ..................................................53

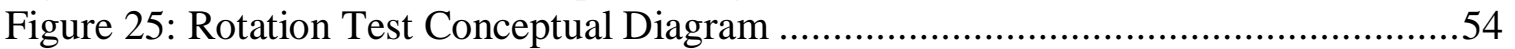

Figure 26: Scaling Test Conceptual Diagram ........................................................5

Figure 27: Viewpoint Angle Test Conceptual Diagram ..............................................56

Figure 28: Translation Images, Indoor 1 ..............................................................5

Figure 29: Translation Plots Indoor 1, a) SIFT Keypoint Matches, b) Center Point Pixel Distances, c) Cross Correlation Btwn Current and Previous Frame, d) Cross Correlation Btwn Current and Model Frame

Figure 30: Rotation Mean Plots, All Scenes, a) Mean of SIFT Keypoint Matches, b)

Mean of Center Point Pixel Distances, c) Mean of Cross Correlation Btwn Current and Previous Frame, d) Mean of Cross Correlation Btwn Current and Model Frame......61 Figure 31: Rotation Images, Indoor 1

Figure 32: Rotation Plots Outdoor 1, a) SIFT Keypoint Matches, b) Center Point Pixel Distances, c) Cross Correlation Btwn Current and Previous Frame, d) Cross Correlation Btwn Current and Model Frame

Figure 33: Rotation Mean Plots, All Scenes, a) Mean of SIFT Keypoint Matches, b) Mean of Center Point Pixel Distances, c) Mean of Cross Correlation Btwn Current and Previous Frame, d) Mean of Cross Correlation Btwn Current and Model Frame......66

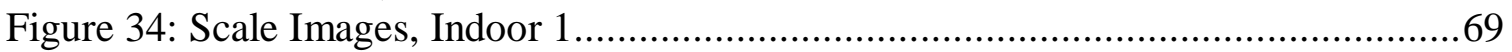

Figure 35: Scale Plots Indoor 1, a) SIFT Keypoint Matches, b) Center Point Pixel Distances, c) Cross Correlation Btwn Current and Previous Frame, d) Cross Correlation Btwn Current and Model Frame.... .70

Figure 36: Scale Mean Plots, All Scenes, a) Mean of SIFT Keypoint Matches, b) Mean of Center Point Pixel Distances, c) Mean of Cross Correlation Btwn Current and Previous Frame, d) Mean of Cross Correlation Btwn Current and Model Frame......71

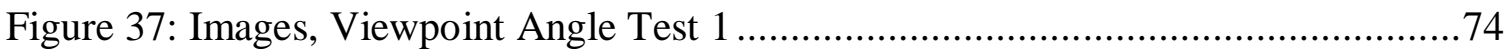
Figure 38: Viewpoint Angle Test 1 Plots, a) SIFT Keypoint Matches, b) Center Point Pixel Distances, c) Cross Correlation Btwn Current and Previous Frame, d) Cross Correlation Btwn Current and Model Frame …………............................................... 75

Figure 39: Images, Viewpoint Angle Test 2 …………….......................................76 Figure 40: Viewpoint Angle Test 2 Plots, a) SIFT Keypoint Matches, b) Center Point Pixel Distances, c) Cross Correlation Btwn Current and Previous Frame, d) Cross Correlation Btwn Current and Model Frame ................................................................77

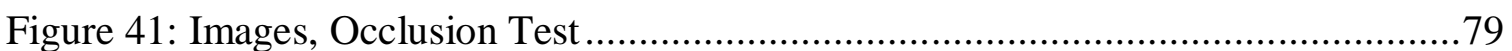

Figure 42: Occlusion Test Plots, a) SIFT Keypoint Matches, b) Center Point Pixel Distances, c) Cross Correlation Btwn Current and Previous Frame, d) Cross

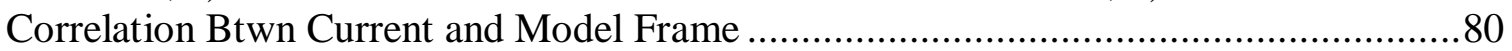

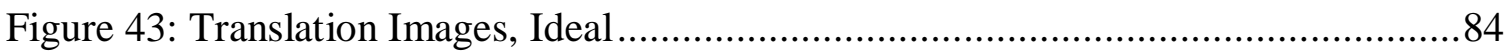

Figure 44: Translation Plots Ideal, a) SIFT Keypoint Matches, b) Center Point Pixel Distances, c) Cross Correlation Btwn Current and Previous Frame, d) Cross

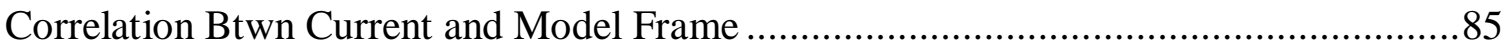

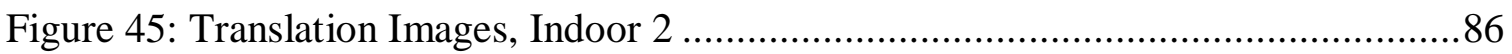

Figure 46: Translation Plots Indoor 2, a) SIFT Keypoint Matches, b) Center Point Pixel Distances, c) Cross Correlation Btwn Current and Previous Frame, d) Cross Correlation Btwn Current and Model Frame 
Figure 47: Translation Images, Outdoor 1

Figure 48: Translation Plots Outdoor 1, a) SIFT Keypoint Matches, b) Center Point Pixel Distances, c) Cross Correlation Btwn Current and Previous Frame, d) Cross Correlation Btwn Current and Model Frame 89

Figure 49: Translation Images, Outdoor 2

Figure 50: Translation Plots Outdoor 2, a) SIFT Keypoint Matches, b) Center Point

Pixel Distances, c) Cross Correlation Btwn Current and Previous Frame, d) Cross Correlation Btwn Current and Model Frame

Figure 51: Translation Images, Outdoor 3

Figure 52: Translation Plots Outdoor 3, a) SIFT Keypoint Matches, b) Center Point

Pixel Distances, c) Cross Correlation Btwn Current and Previous Frame, d) Cross

Correlation Btwn Current and Model Frame

Figure 53: Translation Images, Outdoor 4

Figure 54: Translation Plots Outdoor 4, a) SIFT Keypoint Matches, b) Center Point

Pixel Distances, c) Cross Correlation Btwn Current and Previous Frame, d) Cross

Correlation Btwn Current and Model Frame

Figure 55: Rotation Images, Ideal

Figure 56: Rotation Plots Ideal, a) SIFT Keypoint Matches, b) Center Point Pixel

Distances, c) Cross Correlation Btwn Current and Previous Frame, d) Cross

Correlation Btwn Current and Model Frame

Figure 57: Rotation Images, Indoor 2

Figure 58: Rotation Plots Indoor 2, a) SIFT Keypoint Matches, b) Center Point Pixel

Distances, c) Cross Correlation Btwn Current and Previous Frame, d) Cross

Correlation Btwn Current and Model Frame

Figure 59: Rotation Images, Indoor 2.

Figure 60: Rotation Plots Indoor 2, a) SIFT Keypoint Matches, b) Center Point Pixel

Distances, c) Cross Correlation Btwn Current and Previous Frame, d) Cross

Correlation Btwn Current and Model Frame

Figure 61: Rotation Images, Outdoor 1

Figure 62: Rotation Plots Outdoor 1, a) SIFT Keypoint Matches, b) Center Point

Pixel Distances, c) Cross Correlation Btwn Current and Previous Frame, d) Cross Correlation Btwn Current and Model Frame

Figure 63: Rotation Images, Outdoor 2

Figure 64: Rotation Plots Outdoor 2, a) SIFT Keypoint Matches, b) Center Point

Pixel Distances, c) Cross Correlation Btwn Current and Previous Frame, d) Cross

Correlation Btwn Current and Model Frame

Figure 65: Rotation Images, Outdoor 3

Figure 66: Rotation Plots Outdoor 3, a) SIFT Keypoint Matches, b) Center Point

Pixel Distances, c) Cross Correlation Btwn Current and Previous Frame, d) Cross

Correlation Btwn Current and Model Frame

Figure 67: Rotation Images, Outdoor 4

Figure 68: Rotation Plots Outdoor 4, a) SIFT Keypoint Matches, b) Center Point

Pixel Distances, c) Cross Correlation Btwn Current and Previous Frame, d) Cross

Correlation Btwn Current and Model Frame

Figure 69: Scale Images, Ideal 
Figure 70: Scale Plots Ideal, a) SIFT Keypoint Matches, b) Center Point Pixel

Distances, c) Cross Correlation Btwn Current and Previous Frame, d) Cross

Correlation Btwn Current and Model Frame

Figure 71: Scale Images, Indoor 2.

Figure 72: Scale Plots Indoor 2, a) SIFT Keypoint Matches, b) Center Point Pixel

Distances, c) Cross Correlation Btwn Current and Previous Frame, d) Cross

Correlation Btwn Current and Model Frame

Figure 73: Scale Images, Outdoor 1

Figure 74: Scale Plots Outdoor 1, a) SIFT Keypoint Matches, b) Center Point Pixel

Distances, c) Cross Correlation Btwn Current and Previous Frame, d) Cross

Correlation Btwn Current and Model Frame

Figure 75: Scale Images, Outdoor 2

Figure 76: Scale Plots Outdoor 2, a) SIFT Keypoint Matches, b) Center Point Pixel

Distances, c) Cross Correlation Btwn Current and Previous Frame, d) Cross

Correlation Btwn Current and Model Frame

Figure 77: Scale Images, Outdoor 3

Figure 78: Scale Plots Outdoor 3, a) SIFT Keypoint Matches, b) Center Point Pixel Distances, c) Cross Correlation Btwn Current and Previous Frame, d) Cross

Correlation Btwn Current and Model Frame

Figure 79: Scale Images, Outdoor 4

Figure 80: Scale Plots Outdoor 4, a) SIFT Keypoint Matches, b) Center Point Pixel Distances, c) Cross Correlation Btwn Current and Previous Frame, d) Cross Correlation Btwn Current and Model Frame 


\section{Chapter 1: Introduction}

The consumer electronics industry has driven an increase in both the speed of computer processors, and the number of pixels detectable by digital cameras. This has allowed for the implementation of computer vision algorithms that were purely theoretical in the last century.

More recently hand-held computer electronics, such as cell phones, have reached computational speeds required to perform tasks previously reserved for the desktop computing world, such as running multiple processes or decoding video for playback. A majority of these devices now include multi-megapixel cameras, high resolution displays, and large amounts of flash memory. Also, many include GPS for position data and a relatively uninterrupted connection to the internet via the cellular network. This ever increasing functionality in mobile consumer electronics opens up the possibility of performing new useful tasks through computer vision.

Assuming the functionality of a given computer vision system is that of a modern mobile consumer electronics device, the system can (1) capture real-time video at 20+ frames/sec, (2) perform some level of processing on all or most frames, (3) acquire precise geographical location, and (4) access large databases either locally or via the internet. All of these capabilities lend themselves to a creating a system that is aware of its location and able to recognize its surroundings and the objects in them, by executing various computer vision algorithms. 


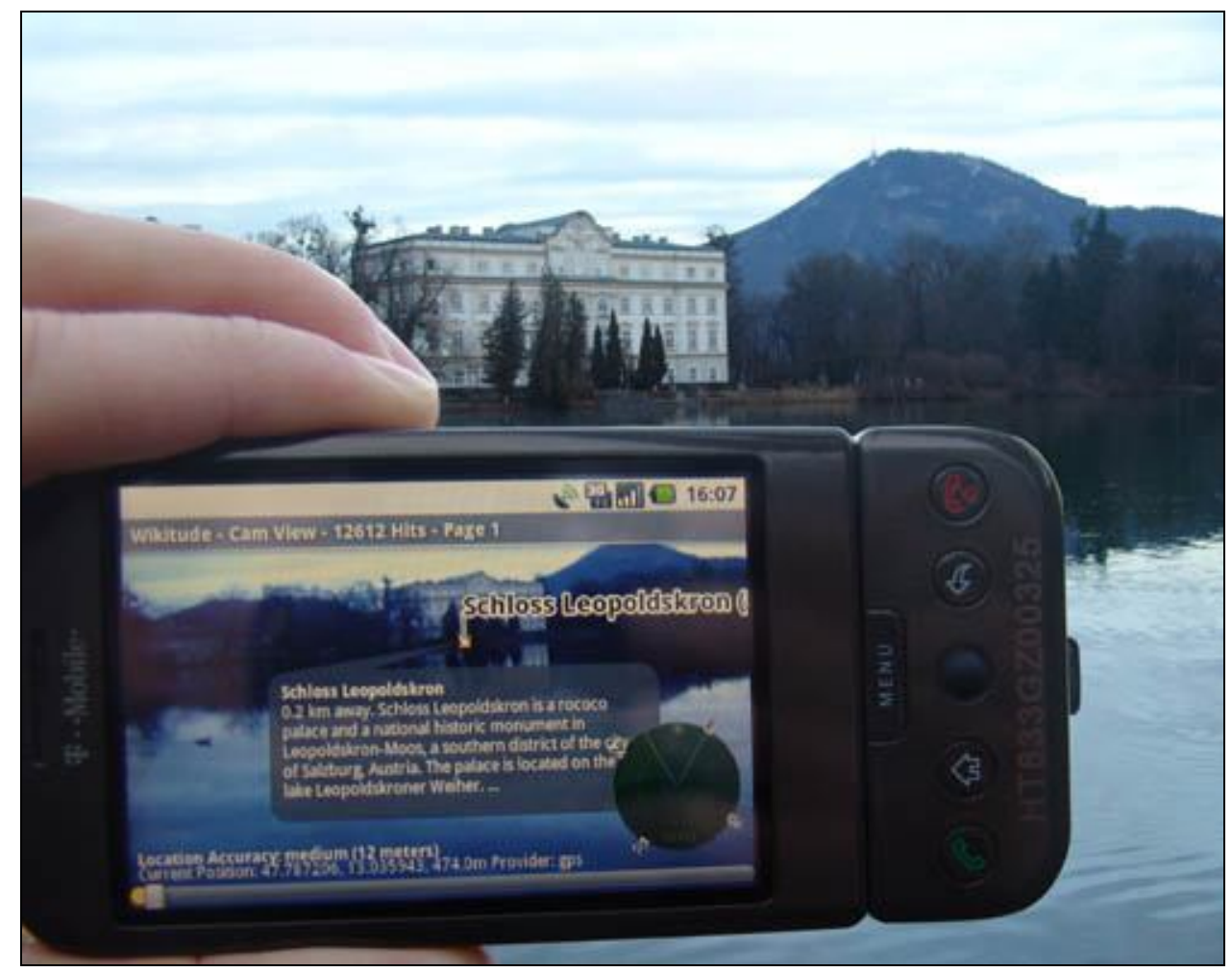

Figure 1: Picture of "Wikitude” Application running on Google Android OS [13]

One scenario that encompasses many of these capabilities is as follows. A traveler comes upon an old castle in Europe. Their cell phone recognizes their location via GPS, and starts to load a database of objects and facts about the area. The traveler holds their cell phone camera up to view the castle from a distance on a hillside (Figure 1).

Displayed alongside the real world image of the castle some text appears: the name of the castle, its location, when it was built, and several internet hyperlinks to the current owner's website, a map of the castle interior, Wikipedia articles, etc. The traveler decides to walk up to the castle wall. Panning the cell phone camera across the exterior of the castle wall hand written notes by previous visitors appear to be physically written on the wall. New notes are easily added to the wall which can be shared publically, or just with a select group of people. 
An application for performing a task similar to this scenario has already been developed and released. On October 20, 2008 Wikitude AR Travel Guide launched with the G1 Android phone and was downloaded about 50,000 times in its first year of release. This application has the basic functionality of recognizing landmarks and displaying text taken from Wikipedia along side it (Figure 1). The popularity of this application is evidence of user demand for these types of Computer Vision driven applications.

\section{Augmented Reality}

The above scenario belongs to the field of computer vision called Augmented Reality (AR). Augmented Reality is a field of computer vision that combines real-world and computer-generated data (virtual reality), where computer graphics objects are blended into real footage in real time. In the case of this scenario, the computer graphic

objects are simply the text displayed to the user over the real footage of the castle. Using a cell phone for AR applications is often referred to as Mobile Augmented Reality, or mobile AR.

In general, an AR system consists of (1) a display, (2) a camera, and (3) a computer. Ideally the user would wear a transparent display in the form of glasses which produce the appearance of overlaid virtual objects on the user's real world surroundings. One or more miniature cameras would be embedded in the frame of the glasses for computer vision. A mobile computer would come in the form of a wirelessly connected handheld device, or a smaller computer embedded in the glasses. A similar setup can be created with today's hardware relatively inexpensively. In Figure 2 a mannequin is seen wearing an AR system consisting of a head-mounted display (HMD), and two small embedded cameras. 


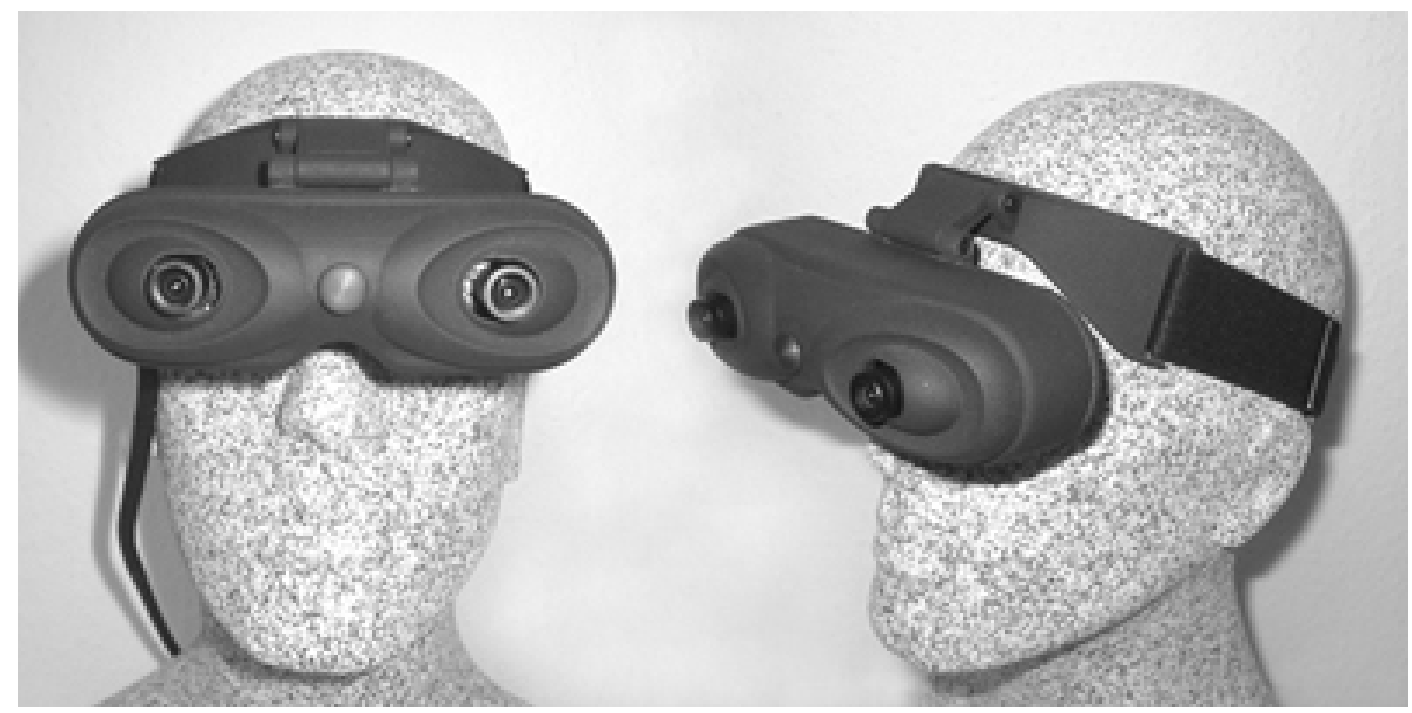

Figure 2: Head Mounted Display (HMD) intended for Stereoscopic AR [14]

One way to define an AR system limits it to the use of HMDs. However a broader definition requires an AR system to posses the following three characteristics [1]:

1) Combines real and virtual data

2) Interactive in real time

3) Registered in $3 D$

The above criteria for an AR system will be used as a qualification for all AR system's discussed in this paper.

\section{Additional Related Applications}

Based on the broad definition of Augmented Reality [1], there are currently many implemented applications for AR. Probably the most common examples in the United States is the "first-down line" in American Football. Sportvision, a company based in New York City, debuted its "1st and Ten" system during a game broadcast on ESPN on September 27, 1998. The cameras recording the football game are part of an AR system 
which tracks the position of the field relative to the cameras. This is most likely done by observing the position of real-world markers, such as the orange marker (top of image in Figure 3) and the painted white lines going across the field. Also, the exact movement of the camera (tilt, pan, zoom, focus) is known. With this information the system is able to draw a virtual yellow line which appears stationary across the field. This system meets all of the requirements to be considered an AR application because it combines real and virtual data, is interactive in real time, and is registered in 3D. The fact that the Super bowl had over 95 million viewers demonstrates how pervasive AR is becoming in average consumer life.

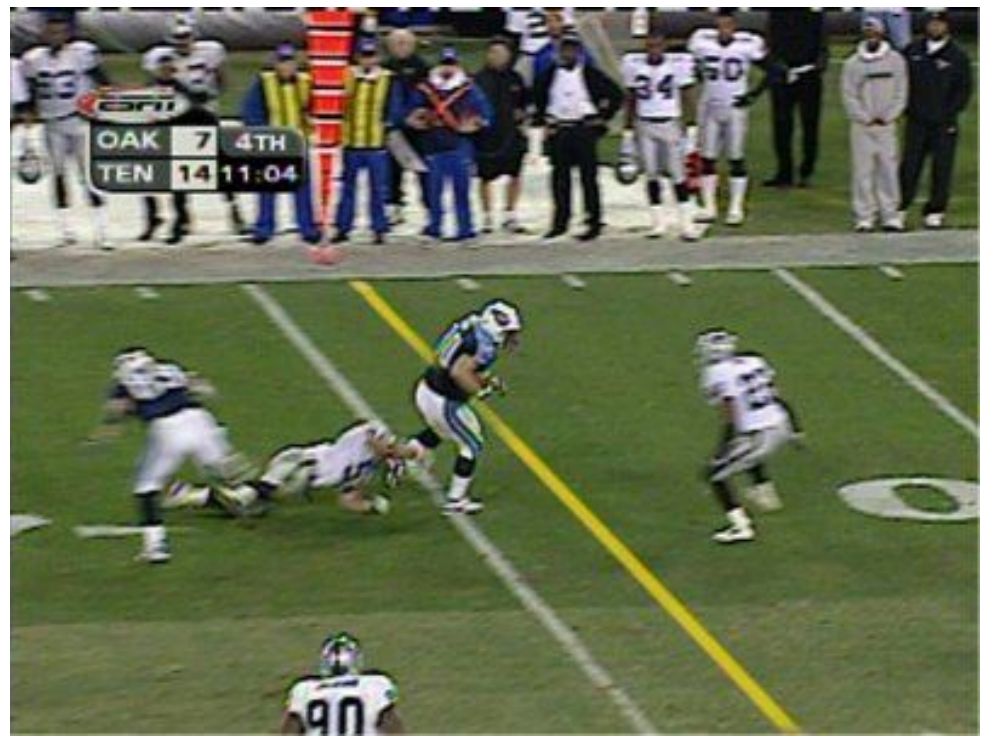

Figure 3: A football players appears to reach for the "yellow line", yet only viewers at home can see it (C) SporTVision) 


\section{Goal of Method Presented}

The goal of this research paper is to create an AR system which can display a virtual 2D image on a real world 3D wall. The method chosen can do this without developing a world model and without a priori information about the environment. In order to perform this task the AR system must (1) track the wall relative to the user and (2) determine the wall's orientation relative to the user to register it in the 3D environment. The paper is organized as follows:

1. In Chapter 2 a survey of related works is presented, ending with a comparison of different operators similar to the two main methods utilized: Maximally Stable Extremal Regions (MSER) and Scale Invariant Feature Transform (SIFT)

2. In Chapter 3 a theoretical derivation of all methods used is presented, starting with the previously developed MSER and SIFT algorithms, followed by a description of the method of this paper

3. In Chapter 4 an experimental setup, testing, results, and discussion is presented through a representative set of the data for Translation, Rotation, and Scale. The complete set of data for these 3 tests can be found in Appendix A. Two secondary tests are presented: Viewpoint Angle Change, and Occlusion

4. In Chapter 5 the discussed results of Chapter 4 are analyzed to present an overall conclusion on the performance of the method presented. 


\section{Chapter 2: Survey of Related Works}

There have been many approaches in AR and other Computer Vision based fields to accomplishing these tasks of (1) tracking and (2) registration. This paper examines possible solutions to this problem that generally break down into two categories defined by the features used for tracking. Unlike the method of this paper, some require a prior information about the environment, and some require the development of a world model:

1) Marker-based tracking

a. Generally requires a priori information about markers

b. Sometimes develops a world model

2) Markerless tracking of invariant image features

a. Requires no a priori information about image features

b. Sometimes develops a world model

The goal of the ideal AR system is to function in any real world environment without requiring the user to annotate that environment in any way. In other words, it is very desirable to have a system that requires no markers to function, because the real world has no markers.

Table 1 provides a brief summary of the specific examples discussed as related works. There are many other examples of marker-based tracking methods, but this survey focuses on the choice of a markerless tracking method.

Table 1: Comparison of Marker-based Tracking and Markerless Tracking

\begin{tabular}{|l|l|l|}
\hline $\begin{array}{l}\text { Basis for } \\
\text { Tracking }\end{array}$ & Marker-based tracking & Markerless tracking \\
\hline Examples & $\begin{array}{l}\text { Corner-based marker tracking, } \\
\text { Color-based stereoscopic } \\
\text { marker tracking }\end{array}$ & $\begin{array}{l}\text { Affinely invariant regions } \\
\text { (MSER), Local image descriptors } \\
\text { (SIFT) }\end{array}$ \\
\hline
\end{tabular}




\begin{tabular}{|l|l|l|}
\hline $\begin{array}{l}\text { Development of } \\
\text { World Model }\end{array}$ & $\begin{array}{l}\text { Requires Stereoscopic Vision } \\
\text { or known marker locations }\end{array}$ & $\begin{array}{l}\text { Can be done with single-camera } \\
\text { vision by structure from motion }\end{array}$ \\
\hline $\begin{array}{l}\text { Development of } \\
\text { Model View }\end{array}$ & $\begin{array}{l}\text { May require mathematical } \\
\text { description of models }\end{array}$ & $\begin{array}{l}\text { Automatic processing of training } \\
\text { images to develop model }\end{array}$ \\
\hline
\end{tabular}

\section{Marker-based Tracking}

In order to augment a scene an AR system must orient itself with respect to its realworld environment. This falls under the requirement that an AR system register itself in 3D. To accomplish this task an AR system must recognize something physical in its environment and track it for registration. An AR system may track natural elements in the physical scene, or may be designed to recognize specially placed "Markers." There are many types of markers, but all have features specifically incorporated to simplify the system's ability to recognize and track them. This reduces the number of variables that the system has to account for, and in general reduces the overall computational complexity of the Computer Vision algorithms.

\section{Marker-based Corner Tracking}

One method of marker-based tracking utilizes markers that consist of known corner positions on a $2 \mathrm{D}$ planar region. The system that utilizes these corner based markers is a robust corner tracker able to deal with occlusion and changes in scale, orientation, and illumination. By computing the homography between known corner positions on a planar pattern and potential planar regions in a video sequence, the system is able to predict corner positions [2]. From the predicted corner positions the system places search windows around the predicted locations of corners and then uses a Harris corner detector to find the corner to within pixel accuracy. This is then used to update the homography to be compared to the next frame. 
From these corner positions the orientation of the paper with respect to the known patterns can be determined. This indirectly orients the system relative to the marker, and through use of the homography, allows for projection of $2 \mathrm{D}$ images onto the $2 \mathrm{D}$ marker plane (Figure 4).

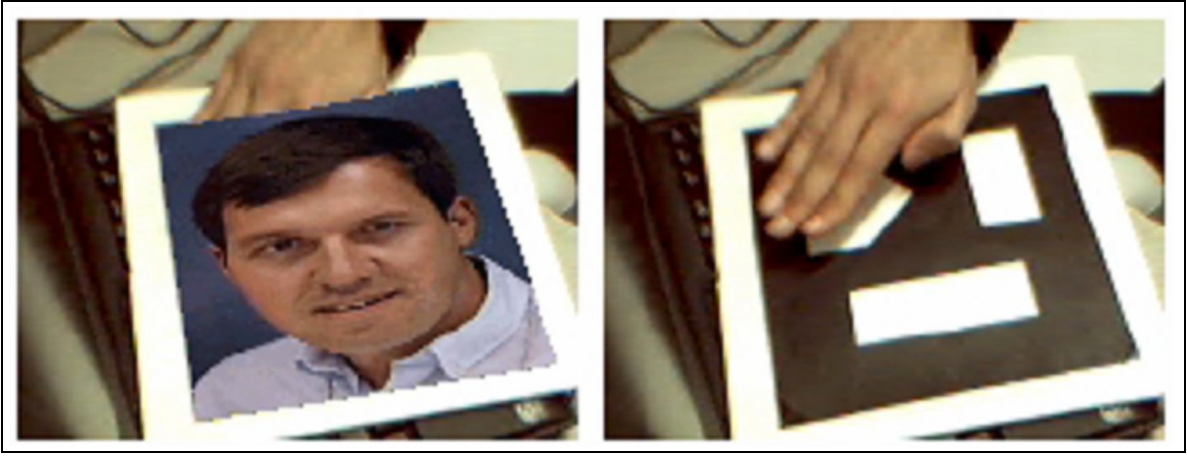

Figure 4: Corner-based marker tracking Successful Augmentation onto a partially occluded pattern [2]

There are several advantages to this corner-based tracking method. First, it reduces the search space to less than 100 points of interest, allowing for a real-time implementation. It also is very insensitive to changes in scale and illumination and fairly insensitive to occlusion as long as more than 10 corners are visible. The biggest disadvantage of the method is the fact that under severe viewpoint rotation, perspective distortions cause corners to appear very close to one another and the system can no longer uniquely identify a particular pattern.

\section{Marker-based Stereoscopic Tracking}

By observing colored markers through a Stereoscopic camera setup, an AR system can precisely calculate the relationship between the world and camera coordinate systems [3]. Two calibrated cameras are used to generate a point cloud of 3D data. The cameras are calibrated for a one-to-one correspondence between pixels from one camera 
to the other, usually based on epipolar lines. This requires the baseline distance between the cameras be fixed. Once a 3D point cloud is generated the distance to each pixel is known.

One system uses several colored markers with known positions in a room. If three or more of these markers are visible to the camera's they can be used to calculate what is called a model view matrix. The model view matrix represents the spatial relationship between two coordinate systems. The model view matrix $M$ is defined so that the equation $\mathrm{c}=\mathrm{Mw}$ holds true, where the position of a point in the world coordinate system is denoted by $\mathrm{w}$, and the position of the same point in the camera coordinate system is denoted by c (x, y, z in Figure 5). When the system is turned on, three blue markers of known position are observed and a model-view matrix $M$ is calculated to geometrically align the real and virtual world coordinates. By continuously tracking the blue markers the system is able to register itself in the surrounding 3D environment.

If the three blue markers of known position $\left(\mathrm{m}_{1}, \mathrm{~m}_{2}\right.$, and $\mathrm{m}_{3}$ in Figure 6$)$ are in the FOV of the cameras and three red markers of unknown position $\left(m_{k}, m_{i}\right.$, and $m_{j}$ in Figure 6) are brought into the FOV of the cameras at the same point in time, the system can determine the position of the three red markers. If the original blue markers leave the field of view (FOV) of the cameras, the three red markers of unknown initial position can then be tracked and used to register the system. The only requirement is that all blue markers and all red markers must be in the FOV of the cameras at some point before the blue markers go out of the FOV. One model-view matrix $\mathrm{M}_{1}$ is developed to translate between the world coordinate system and the camera coordinate system, and a second model-view matrix $\mathrm{M}_{2}$ can be defined as the relationship between the world coordinate 
system and the new red marker coordinate system. If the markers of known position (blue markers) go out of the cameras FOV, but the markers of unknown initial position (red markers) are still visible, the system can register itself accurately.

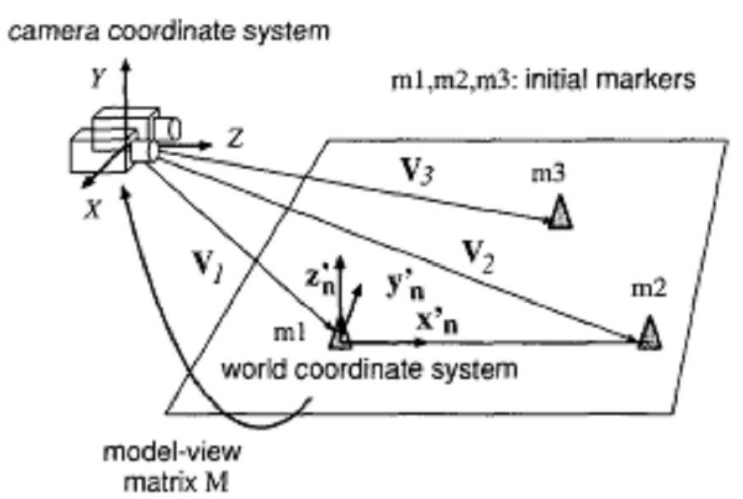

Figure 5: Relationship between the world and camera coordinates. [3]

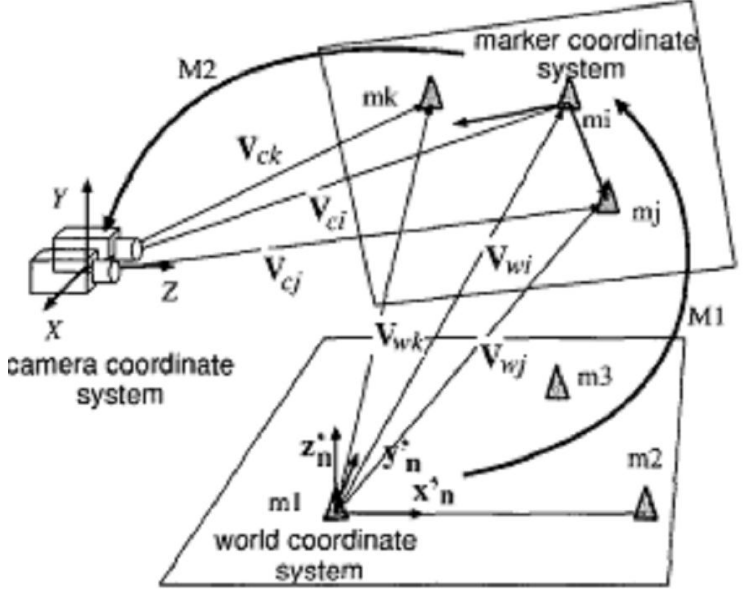

Figure 6: Relationship between the marker and camera coordinates. [3] 


\section{Markerless Tracking: Invariant Image Features}

Although marker-based tracking approaches offer several advantages, mainly computationally, the ideal AR system should not require the use of markers because the real world is not annotated with markers. In the example of Mobile AR, it is impractical for a user to manually place markers in their environment. The system is constantly in new places and a typically application is more concerned with identifying general interest points than previously placed markers. To accomplish this task an AR system must recognize something physical in its environment not known a priori and track it for registration. In this case an $\mathrm{AR}$ system will track natural elements in the physical scene using methods generally known as markerless tracking.

The fact that the interest points are not known a priori does not exclude the possibility of object recognition through the use of other previously known information, such as object models. Because of the lack of information about the interest points, markerless tracking systems must exploit what are typically referred to as invariant image features. These are image features that tend to retain invariant characteristics despite possible changes in viewpoint, scale, rotation, and lighting. Some examples of points like this include corners, planar surfaces, or other geometrically defining features in an environment.

\section{Stereoscopic Plane Tracking}

As was the case with marker-based stereoscopic tracking, stereoscopic vision on in AR system allows for the world and camera coordinate systems to come into complete correspondence. An AR system with stereoscopic vision can generate a 3D point cloud of 
data and used to detect and locate walls relative to the cameras. The Random Sample Consensus (RANSAC) algorithm can fit numerous geometric models to a set of data, such as a plane. RANSAC works by randomly selecting the number of data points required to create an instance of the model, in this case three points to make a plane. It assumes that the selected points are inliers, and all other points are compared to the model to see how closely they fit. If they are within a chosen threshold they are also considered inliers. The process repeats iteratively and the model that has the most inliers is chosen to be the correct model, making the algorithm insensitive to outliers, and more accurate with more iterations.

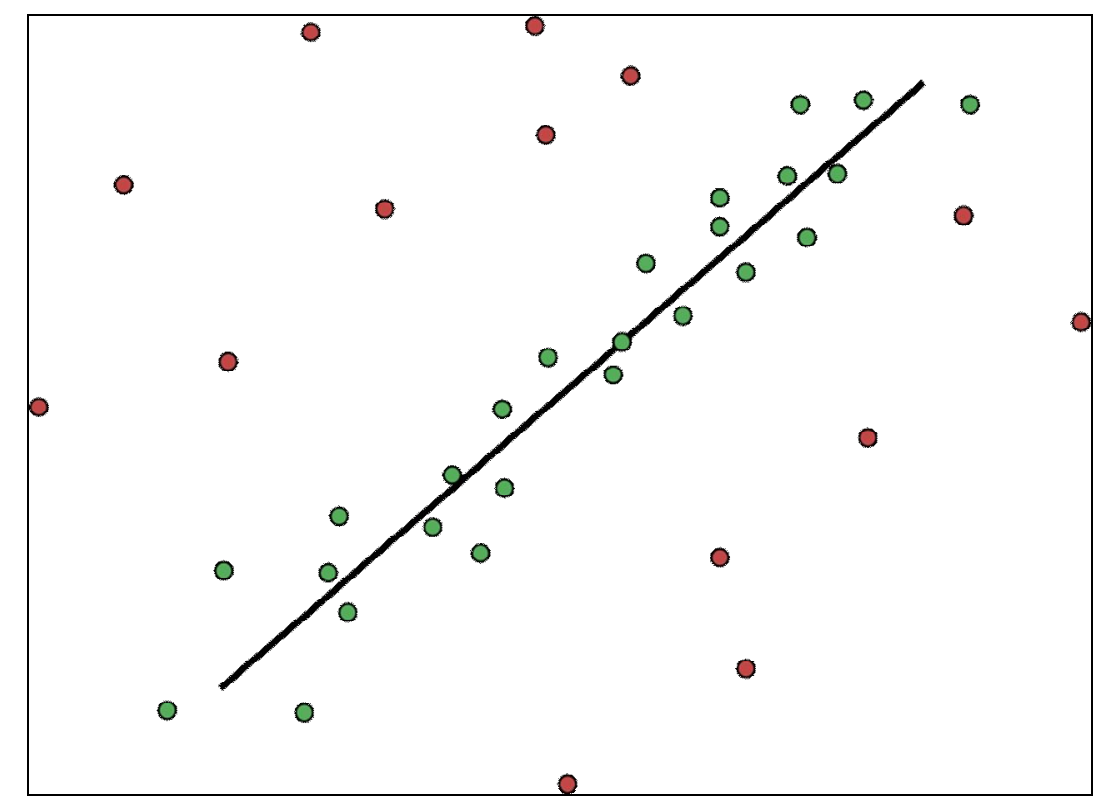

Figure 7: Fitted line with RANSAC, outliers (red) have no influence on the result.

Fitting a plane to a point cloud of walls in a room directly using the RANSAC algorithm only works if there are sufficient number of data points [4], which may not be the case with a low level of texture on the surface of the wall. If different regions of the wall are very similar it's impossible to distinguish them from one another. This causes 
the algorithm that forms the point cloud to throw out large regions of pixel data, essentially making the walls invisible to the system (Figure 8a).

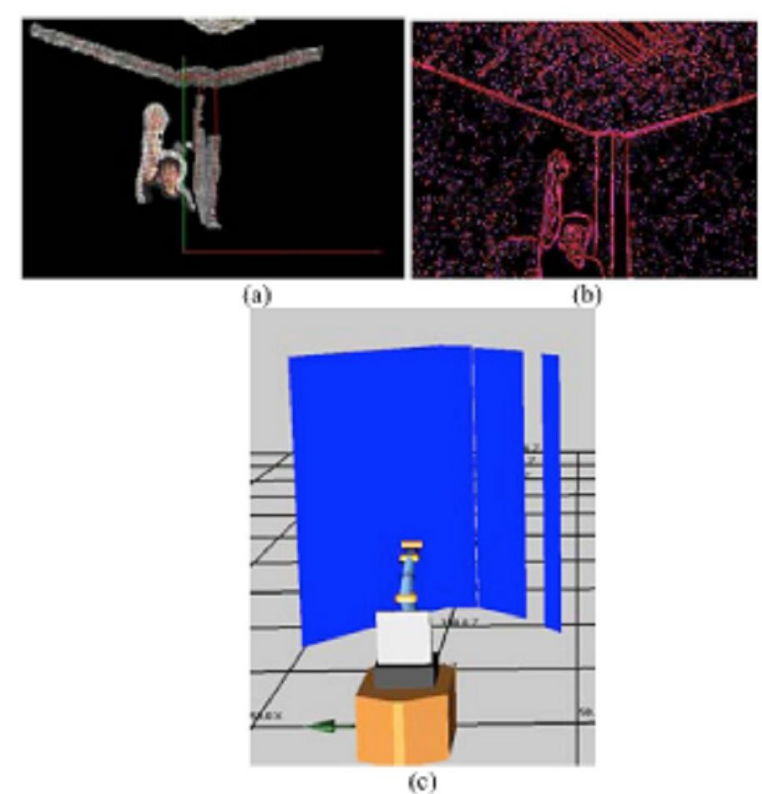

Figure 8: a) the point cloud generated by a stereo vision camera, in which there is no texture on the walls themselves, b) the edges detected in 2D, c) the walls without contradiction. [5]

Research in the field of robotics has lead to the definition of Simultaneous Localization and Mapping (SLAM) which refers to a system building up a map in an unknown environment while keeping track of current position, through sensory information and location data. This problem is non-trivial because, although the robot has precise knowledge of its own movements as well as external sensors to monitor its environment, in a closed loop algorithm small errors in localization can accrue over time and disrupt the stability of the system causing inaccuracies in the mapping. To compensate for this, SLAM algorithms will typically recognize old features and reregister that part of the map so the two instances of that feature are only represented once in the map. Like in the AR system described by this paper, SLAM algorithms must register obstacles such as walls in order to build a map. 
One method for SLAM in robotics uses stereoscopic data to solve the problem of "textureless" walls by observing edges in the ceiling [5]. The system starts by creating a set of "hypothetical walls" based on the edges in the ceiling. Then, stereoscopic data is used to either confirm or reject the walls as real. This is effective because, although the walls may be textureless and therefore provide little stereoscopic data, the amount of stereoscopic data present is enough to confirm hypothetical walls as real walls. As the robot moves around a map is built based on the motion of the robot in relation to the real walls. Hypothetical walls are considered to be real until they are confirmed to be false. The advantages of this method are that it requires no a priori knowledge of the environment, and is relatively computational simple as compared to the RANSAC method of detecting a textured wall. However, because it is part of a SLAM algorithm, it does require a world model to be developed.

\section{Affinely Invariant Region Detectors}

In order to use natural parts of a scene instead of physical markers a method that finds exactly the same region from frame to frame is required. A region that is invariant to changes in scale and rotation is said to be partially affinely invariant [6]. These regions also tend to be invariant to illumination changes, which is the third requirement for invariance between frames.

These regions are accurate models of 2D planes. A feature vector can be defined for each region so they can be easily distinguished from one another. This allows for easy tracking regardless of large changes in motion, rotation, and scaling. By comparing matched regions between frames, an affine transform can be computed to augment the region. As long as the graphic used to augment the region is placed close to the region, 
perspective effects can be ignored. This is because the region and the immediate area surrounding it are affinely invariant, so a graphic transformed by an affine transform will appear to be on that affine region as long as it is placed on top of that region.

There have been many efforts made in comparing the performance of different affine invariant region detectors. In [6], six different region detectors are compared:

1. Harris-Affine

2. Hessian-Affine

3. Maximally Stable Extremal Region (MSER)

4. Intensity Extrema-Based Region (IBR)

5. Edge Based Region (EBR)

6. Salient Regions

A brief description of several of these follows.

The EBR Detector starts at a Harris-corner point and then exams near by edges using the Canny edge detector [7]. Parallelogram shaped regions are built around these initial parameters by detecting other edges that intersect with the initial edge, and cause the Harris-corner point to be enclosed near the center of the edges. The IBR Detector starts by detecting intensity extrema at different scales [8]. Areas on rays starting at the extrema point are evaluated. A function has been developed in [8] that is invariant to affine and linear illumination transformations at its extremum. The function consists of taking the absolute value of the difference of the intensity of the current pixel being examined on the ray and the intensity value at the extrema. This is divided by the integral of the same quantity across all locations on the ray. The region found is defined as the area enclosed by the extremum of the function, centered at the intensity extrema. This results in an elliptically defined region.

Both the Harris-Affine and the Hessian-Affine Detectors produce an elliptical description of their respective regions [9]. Interest points are detected using either the 
Harris corner detector [10] or the Hessian matrix. The Laplacian operator is used to create multiple scales of the input image, to create scale invariance. A second-moment matrix is developed to describe the shape of the region.

The MSER Detector begins by taking different thresholdings of an image to produce a set of connected components at each level of thresholding [11]. The rate of change in the growth of the area of these regions is observed, and local regions that vary slowly are considered to be "maximally stable." This produces a 'blob' shaped region, which can be represented by a second-moment ellipse. The ellipse then represents the shape, size and orientation of the MSER. Under the condition of illumination change the MSER detector is relatively unaffected because the thresholding operation finds areas that are relative to one another regarding illumination.

In [16], the six different region detectors being compared were evaluated in the following areas:

1. Region density - the number of regions detected in a scene

2. Region size - area of the regions detected

3. Viewpoint change - change in angle on the scene

4. Scale change - change in scale of the scene

5. Blur

6. Light change

For region density, all of the detectors vary greatly depending on the scene being observed. However the MSER tends to find many regions in structured environments, like those including walls. In terms of region size, the MSER tends to find many small regions, making it less sensitive to occlusion. With viewpoint changes, the MSER performs the best out of all 6 detectors. With scale change, the MSER performs well, second only compared to the Hessian-Affine detector. The only area the MSER is weak in is blurring, performing among the lowest compared to the other detectors. Finally, the 
MSER was most invariant to changes in lighting. Arguably, the MSER detector is the most robust to the most types of changes. This is why the MSER detector was selected for the method of this paper.

\section{Local Image Descriptors}

Local descriptors are different methods of characterizing small regions in an image so that those same regions can be identified in new images of the same scene. There are several main approaches that have been taken to solving this problem [12]:

1. Distribution Based Descriptors

- Scale-Invariant Feature Transform (SIFT)

- Gradient Location and Orientation Histogram (GLOH)

- Spin images

- PCA-SIFT

- Shape context

2. Spatial-Frequency Techniques

- Complex filters

3. Differential Descriptors

- Differential invariants

- Steerable filters

- Moment invariants

As in the case of affinely invariant region detectors, a comparison of the above local descriptors has been made in [12]. A brief description of the different categories of descriptors follows.

Distribution based descriptors utilize histograms to build identifiable feature vectors for matching purposes. Typically, these histograms are based on local shape features around some point of interest. Spatial-frequency techniques convert an image to the frequency domain, and examine changes in frequency and orientation to describe regions. Differential descriptors approximate the neighborhood around a point of interest by computing different order derivatives around that point. 
The SIFT method was found to perform second only to the GLOH method in most test categories which include rotation, scale, blur, and changes in illumination [12]. The GLOH method performs only slightly better than SIFT, and because SIFT is better known and more often implemented, it is chosen over the GLOH method.

\section{Scale-Invariant Feature Transform (SIFT)}

The SIFT method transforms an image into a large collection of local feature vectors, each of which is invariant to image translation, scaling, and rotation, and partially invariant to illumination changes and affine or 3D projection [13]. The first step in the transform is to convolve the image with Gaussian filters at different scales, and then take the difference of successive Gaussian-blurred images. Keypoints are then taken as maxima or minima of the Difference of Gaussians (DoG) that occur at multiple scales. This scale-space extrema detection produces too many keypoint candidates, some of which are unstable. Therefore, the next step in the algorithm is to perform a detailed fit to the nearby data for accurate location, scale, and ratio of principal curvatures. Poorly localized points and points with low contrast will be removed after through this detailed fit, reducing the number of noisy keypoints (Figure 11).

One method uses SIFT to create a world model by first applying the transform to multiple model images from unknown viewpoints of the same scene [14]. A robust wide baseline matching technique is then applied to find correspondences between two model images at a time, resulting in what is essentially stereoscopic imaging through the use of one camera. The next step is non-trivial, in which multiple "stereoscopic" views of the scene are processed to find the structure of the environment relative to the motion of the camera, creating the world model. To update the world model relative to the motion of 
the camera SIFT key points from the current frame are matched to the model images obtained in the first stage of the system.

The SIFT method has the advantage of requiring no a prior knowledge of the environment. Additionally, it does not require the development of a world model, but can be easily used to develop one. The world model can be developed using only a single camera, and without using SLAM (which requires precise knowledge of the systems location at all times). 


\section{Chapter 3: Theoretical Derivations}

The main goal of this work is to develop a method of detecting and tracking invariant

image features by combining the advantages of the MSER detector and the SIFT detector. The MSER detector is used to segment out planar regions in an image, and then SIFT is used to identify invariant interest points. These points are stored as a model view of the planar region, and thus the region can be tracked and successfully augmented. This chapter is concerned with describing in detail all methods involved in creating this successful augmentation.

To perform an affine transformation between two images, at least three affinely invariant points must be matched between these two images. To be affinely invariant these points must be invariant to:

1. Translation

2. Rotation

3. Scaling

Both the MSER detector and the SIFT detector produce descriptors of regions that are affinely invariant (or, more precisely, covariant) to the changes described above. The following portion of the chapter explains the advantages of using MSER and SIFT in conjunction. Table 2 outlines the similarities and differences between the two methods to help clarify the discussion. Also, Table 3 at the end of the discussion provides a list of different symbols used in the discussion. 
Table 2: Comparison of MSER and SIFT Region Detectors

\begin{tabular}{|l|c|c|}
\hline Detector & MSER & SIFT \\
\hline Region Shapes & $\begin{array}{c}\text { Continuously connected pixel } \\
\text { regions or "Blobs" }\end{array}$ & $\begin{array}{c}\text { Circular image regions or } \\
\text { "Keypoints" }\end{array}$ \\
\hline $\begin{array}{l}\text { Region } \\
\text { Representations }\end{array}$ & $\begin{array}{c}\text { Maximum or minimum image } \\
\text { intensity relative to overall } \\
\text { frame }\end{array}$ & $\begin{array}{c}\text { Maximum or minimum image } \\
\text { intensity relative to overall } \\
\text { frame }\end{array}$ \\
\hline $\begin{array}{l}\text { Region } \\
\text { Eescriptors }\end{array}$ & $\begin{array}{c}\text { 5 parameters / ellipsoid: } \\
\text { X center, y center, (2) } \\
\text { independent variance } \\
\text { components, mean }\end{array}$ & $\begin{array}{c}\text { Circular regions } \\
\text { x center, y center, scale (radius), } \\
\text { orientation (angle), descriptor } \\
\text { (128 elements) }\end{array}$ \\
\hline $\begin{array}{l}\text { Region Types } \\
\text { Detected }\end{array}$ & $\begin{array}{c}\text { Textured, planar surfaces } \\
\text { Textured, planar surfaces and } \\
\text { feature points of general 3D } \\
\text { objects }\end{array}$ \\
\hline
\end{tabular}

The MSER detector finds regions that tend to be planar, as long as the distance from the camera to the observed scene is great enough that projective effects can be neglected. This is sometimes cited as a weakness of the MSER and other similar methods when compared to the SIFT detector [14], because it less generalized for tasks such as 3D object recognition. The SIFT detector is not limited to finding planar regions, but is also capable of finding general feature points of 3D objects. However, because the goal of this work is to find planar regions for augmentation, this feature of the MSER proves to be desirable.

\section{Advantages of Using MSER and SIFT in Conjunction}

Using the MSER detector on an image is essentially equivalent to finding the planar regions in that image. By using the MSER detector the search space is reduced for finding SIFT keypoint matches from frame to frame. By selecting a particular ellipse, a specific planar region is chosen for augmentation. The SIFT points that are enclosed 
within the ellipse are considered to be features of the planar surface that that ellipse represents.

In order to identify an object, including a planar region, a SIFT detector must analyze one or more training images to create a "model view" $(M)$ of that object. The SIFT points detected in the model view $\left(S K_{M}\right)$ are used to identify instances of that same object in other images. The SIFT detector without training images has no ability to identify objects. By identifying planar regions through the use of the MSER detector, the system can segment out planar regions as the objects of interest in a scene, allowing the SIFT detector to form a model view of that scene.

Another advantage of using MSER and SIFT in conjunction is additional verification that a region has been successfully augmented. When a match between SIFT points from the model view $\left(S K_{M}\right)$ and SIFT points from the current tracked region $\left(S K_{i}\right)$ is made, an Affine transform is computed $\left(A_{i}\right)$. The MSER detector is then used to find representative ellipses in the current tracked region of the image $\left(E_{i}\right)$. The affine transform $\left(A_{i}\right)$ computed from the current SIFT points $\left(S K_{i}\right)$ is used to transform the center point of the ellipse from the model view frame $\left(C_{M}\right)$ to the new frame. If the transformed center point is close to the center point of the new ellipse, the affine transform is considered to be valid.

Another advantage of using MSER and SIFT in conjunction is faster and more robust tracking of the planar region of interest. The major axis of the representative ellipse found through the MSER detector is used to define the dimensions of a search space. This search space is the only region in the next frame that the SIFT detector will 
operate on. By limiting the search of the SIFT detector to this smaller sub-space, the speed of the overall method is increased.

Table 3: Symbols and their Meaning

\begin{tabular}{|c|c|c|c|c|c|}
\hline \multirow{2}{*}{ Symbol } & \multirow{2}{*}{ Meaning } & \multicolumn{4}{|c|}{ Variations } \\
\cline { 3 - 6 } & Current & Previous & Model & Predicted \\
\hline $\mathrm{A}$ & Affine transform & $A_{i}$ & $A_{i-1}$ & - & - \\
\hline $\mathrm{F}$ & Frame & $F_{i}$ & $F_{i-1}$ & $F_{M}$ & - \\
\hline $\mathrm{M}$ & Model View & - & - & - & - \\
\hline $\mathrm{R}$ & Region & $R_{i}$ & $R_{i-1}$ & $R_{M}$ & $\hat{R}$ \\
\hline $\mathrm{E}$ & Representative Ellipse & $E_{i}$ & $E_{i-1}$ & $E_{M}$ & $\hat{E}$ \\
\hline $\mathrm{SK}$ & Set of SIFT keypoints & $S K_{i}$ & $S K_{i-1}$ & $S K_{M}$ & - \\
\hline $\mathrm{S}$ & Search Space & $S_{i}$ & $S_{i-1}$ & $S_{M}$ & $\hat{S}$ \\
\hline $\mathrm{C}$ & Center Point & $C_{i}$ & $C_{i-1}$ & $C_{M}$ & $\hat{C}$ \\
\hline
\end{tabular}




\section{Maximally Stable Extremal Region (MSER) Detector}

The Maximally Stable Extremal Region (MSER) Detector was originally developed to perform wide baseline stereo matching, or the issue of establishing correspondence between images of a scene from different viewpoints MATT []. It is affinely invariant to illumination changes, very stable, and detectable at multiple scales. It has also been found to be very robust to changes in viewpoint, scale, rotation, and lighting when compared to other affine region detectors [6]. The method finds hundreds of extremal regions, dozens of which are maximally stable. The regions are made up of continuously connected areas of pixels, but are typically represented through the use of a moment ellipse, which defines both the size and orientation of the region, as well as its xy-location.

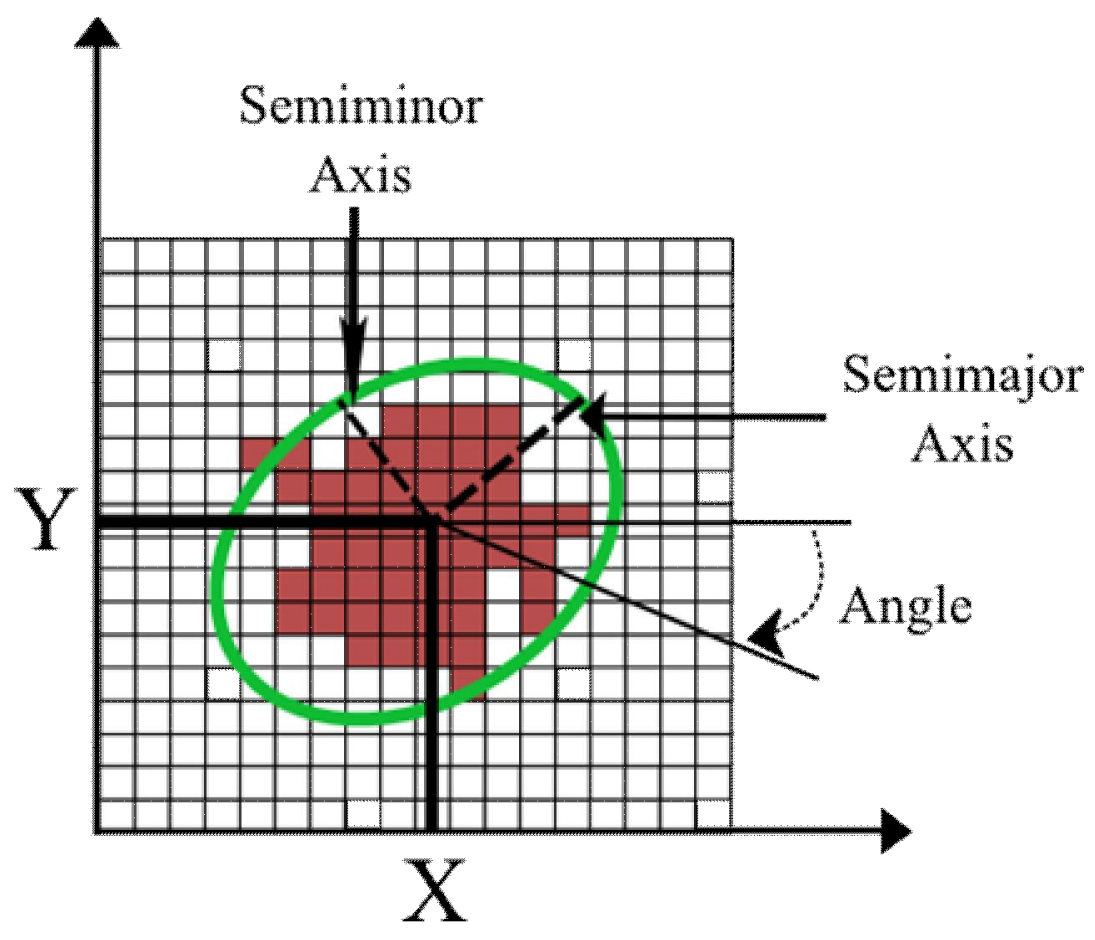

Figure 9: MSER Defining Features 
To find MSERs a thresholding of a grey-scale image is taken at all possible levels. This produces a series of images that start as all "white," and end as all "black" as the level of thresholding increases. Black regions start to grow in areas corresponding to minima's in the image. Eventually two minima will grow large enough that they merge. Maximal regions are considered to be the set of all connected regions of all frames. Minimal regions can be detected by inverting the value of all pixels in the image and repeating the same process.

In order to identify these regions, the following steps are performed:

- Pixels are sorted by intensity value, and then placed in the image in either ascending or descending order. The progression of images in Figure 10a through Figure 10b illustrates the placement of pixels by increasing intensity value.

- As regions merge, a list is created noting which regions are connected and their area. As connected regions merge, the smaller one is considered to be part of the larger one. This merging operation is shown in Figure 10 with regions 1 and 3 in b) merging to become region 1 in c), and regions 4 and 5 in d) merging to become region 4 in e).

- The area of the connected components is stored as a function of intensity. The rate of change of the area of a region is computed, and intensity levels that are local minima of the rate of change in area are considered to be maximally stable 


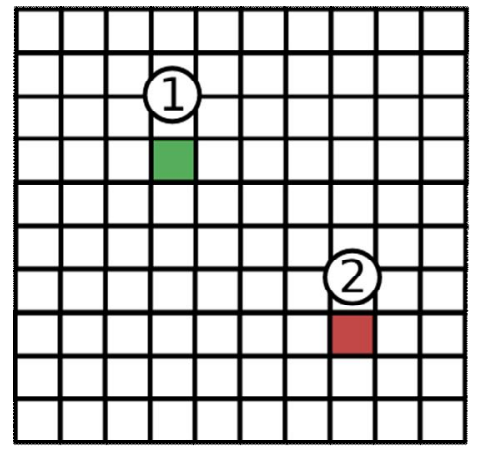

a)

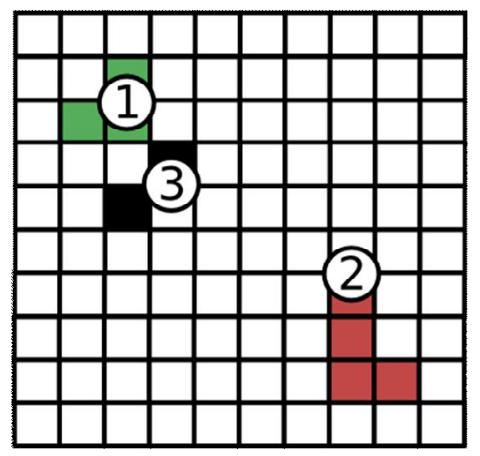

b)

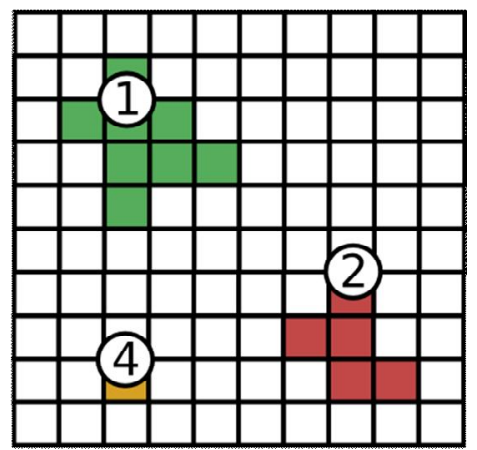

c)

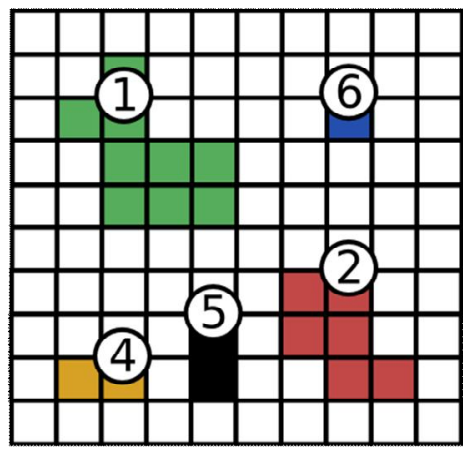

d)

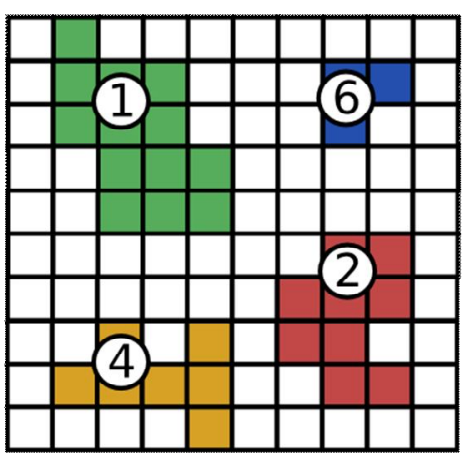

e)

Figure 10: Extremal Regions at Different Thresholds, a) Two minima appear at a low thresholding, b) Minima grow as another region appears at a higher thresholding, c) regions 1 and 3 merge and region 4 appears, d) regions 5 and 6 appear, regions 4 and 5 merge 


\section{Scale-Invariant Feature Transform (SIFT)}

The Scale-Invariant Feature Transform, or SIFT algorithm, is a method developed to find keypoints in an image that are affinely invariant to changes in translation, scale, rotation, and to some extent illumination. Depending on input parameters and image resolution, the method can find anywhere from hundreds to thousands of SIFT keypoints in a given image. For instance, in the method of this paper a camera resolution of 1024x768 produces several thousand SIFT keypoints. SIFT keypoints are circular, and are described by their xy-center point, scale (radius), and orientation as illustrated in Figure 11.

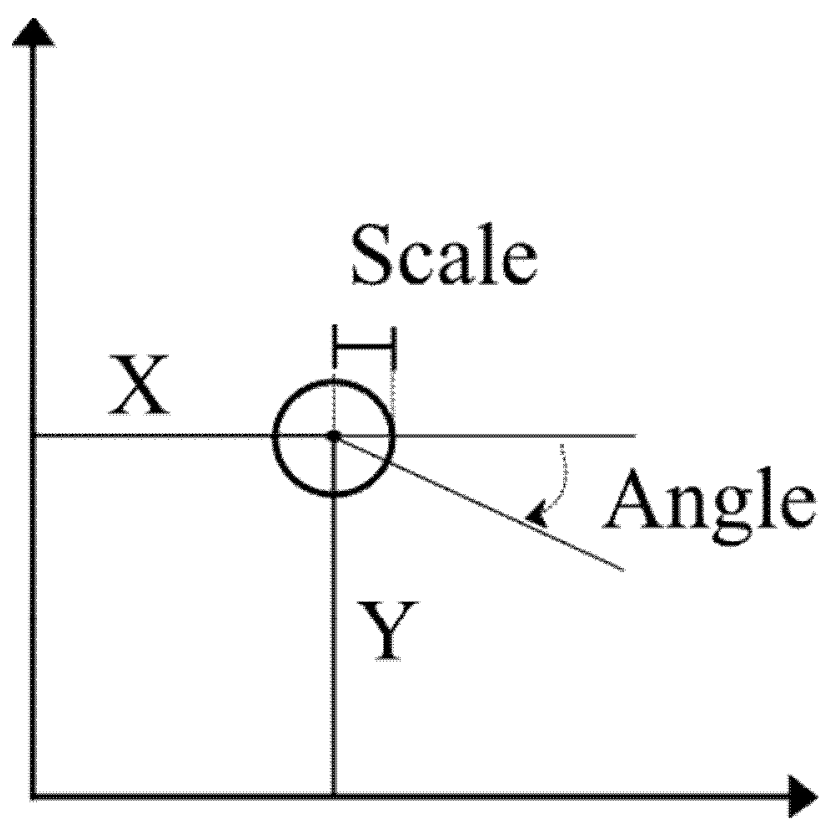

Figure 11: SIFT Keypoint Defining Features 
The method used to detect and describe the SIFT keypoints follows.

\section{Scale Space Extrema Detection}

The first stage of the SIFT algorithm is concerned with identifying possible regions of interest (ROI), which will have to be filtered through and defined more accurately in subsequent steps of the method. In order to perform this preliminary region of interest search, a "scale space" function is created. A scale space function is one that produces multiple versions of the same images at different scales of blurring, and makes the method invariant to scale changes. In this case, scale refers to the resolution of the image. Since it is not possible to increase the resolution of an image, the scale space function decreases the resolution by blurring the image at different levels. It has been found that all forms of the scale space function must be based on the Gaussian blur function [9]. Therefore the scale space function of the following form is used:

$$
\mathrm{L}(x, y, \sigma)=\mathrm{G}(x, y, \sigma) * \mathrm{I}(x, y)
$$

Where $\mathrm{G}(x, y, \sigma)$ is a Gaussian function with variable scaling determined by $\sigma$, and $\mathrm{I}(x, y)$. is the image. The image is convolved with the Gaussian function at a chosen scale, or level of blur, to produce the scale space function $\mathrm{L}(x, y, \sigma)$.

To find stable locations in the image a Difference of Gaussians (DoG) is taken by subtracting the set of scale space function images at adjacent scales as illustrated in Figure 12. Even spacing in scale is achieved by selecting a constant factor $\mathrm{k}$ to multiple the scale factor $\sigma$ by. The DoG function takes the form:

$$
\mathrm{D}(x, y, \sigma)=\mathrm{L}(x, y, \mathrm{k} \sigma)-\mathrm{L}(x, y, \sigma)
$$


Each time the scale $\sigma$ is doubled by the factor k, a new "octave" has been reached.

To maximize the number of DoG images generated, $\mathrm{k}$ is chosen so that an integer number of DoG images are generated per octave.

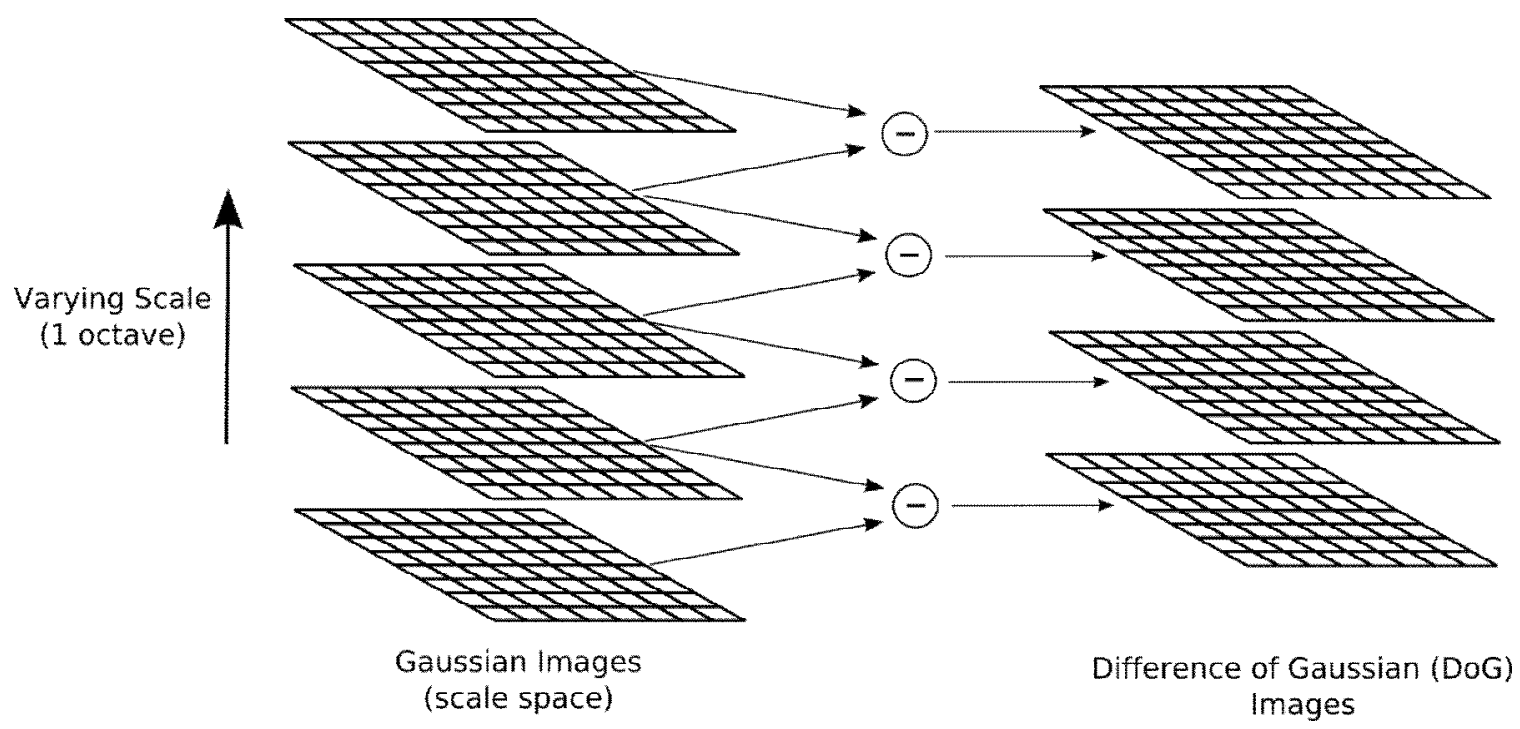

Figure 12: 1-Octave of Difference of Gaussian (DoG) Images

An advantage to taking the DoG is that it is a close approximation of the Laplacian of Gaussian, which has been found to be more invariant to images changes than other image functions such as the Hessian, or Harris corner detector [15].

At this point a set of DoG images have been created through equation (2). In order to identify keypoints the local maxima and minima of each image must be detected. This is done by comparing each pixel in the set of DoG images to its 26 closest neighbors: 8 at the same scale, 9 at one scale above, and 9 at one scale below the current scale (Figure 13). A point is now considered a keypoint if it is either lower in value or higher in value than all 26 of its neighbors. 


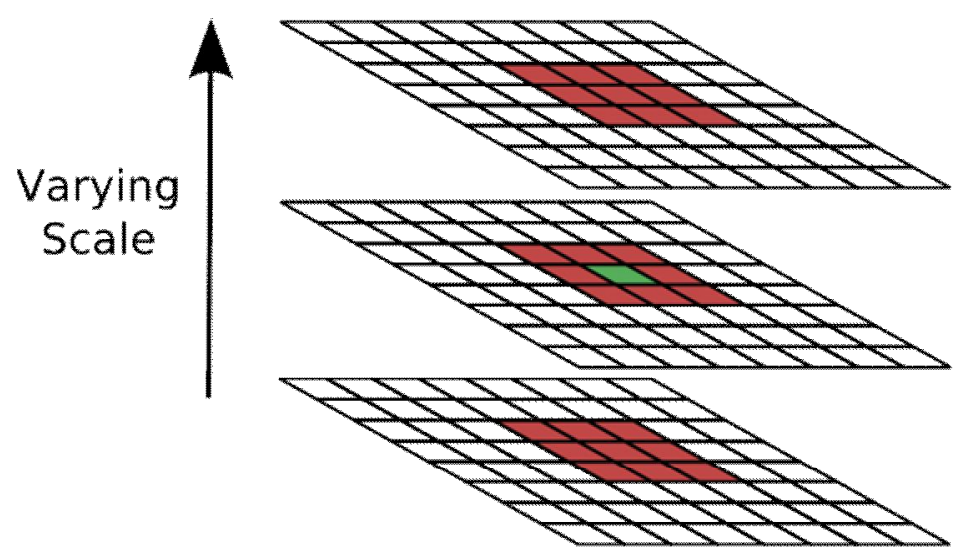

DoG Images

Figure 13: Search for Maxima and Minima by Comparison of Scale Space Neighbors

This process is repeated at multiple octaves, typically at least 3.

\section{Keypoint Localization}

The keypoints generated from the maxima and minima found in the first stage of the SIFT algorithm will contain noisy points that must be filtered out. There are two types of noisy points that are eliminated: those with low contrast, and those that are poorly localized. To deal with both types of noise, the points are fitted to local pixel data to define their location, scale, and ratio of principle curvatures.

The original implementation of SIFT [13] localized keypoints simply by defining the center point to be the pixel location of the maxima or minima point found in the first stage of the method. However, more recent implementations fit a quadratic function to the surrounding data to determine the true location of the maxima or minima. In order to perform this fitting a Taylor expansion is taken of the DoG function [16]:

$$
D(x)=D+\frac{\partial D^{T}}{\partial x} x+\frac{1}{2} x^{T} \frac{\partial^{2} D}{\partial x^{2}} x
$$


Where $\mathrm{x}=(\mathrm{x}, \mathrm{y}, \sigma)^{\mathrm{T}}$ is the offset in location from the original extrema location. The derivative of the Taylor expansion form of the DoG function is taken, and set to 0 in order to find the new location of the extrema:

$$
\hat{x}=-\frac{\partial^{2} D^{-1}}{\partial x^{2}} \frac{\partial D}{\partial x}
$$

Now that the actual location of the extrema is known, equation (4) is substituted into (3) to find the value of the DoG at the extrema. Extrema locations that yield a low value of the DoG function are considered to be low contrast points. They are eliminated by applying a threshold to all points with a low value of $|D(\hat{x})|$. Figure 14 shows the process of noisy points being eliminated. 


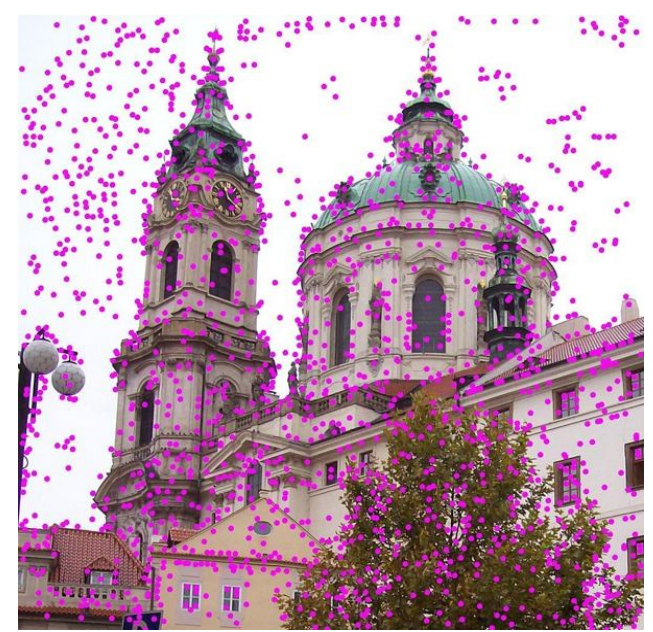

a)

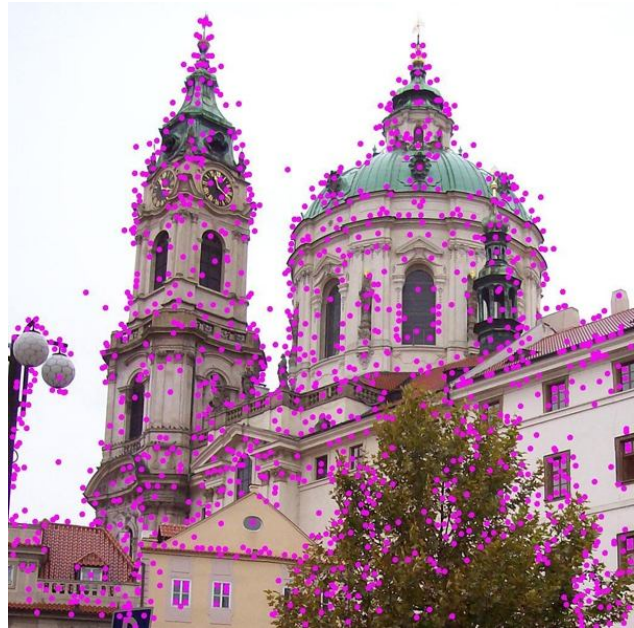

b)

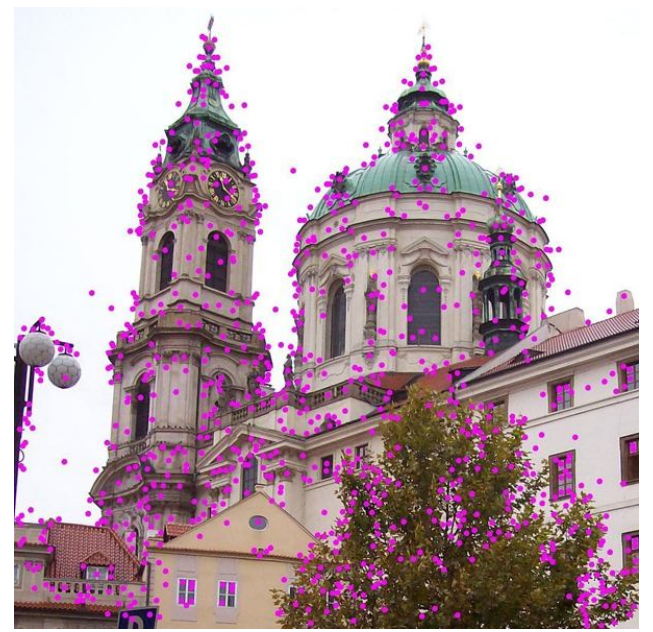

c)

Figure 14: SIFT Keypoints at Various Stages of Filtering a) Scale space extrema are detected by the SIFT algorithm, b) Keypoints of low contrast are filtered out, c) Keypoints that are poorly localized are filtered out resulting in final set of Keypoint locations

To eliminate noisy points that are poorly localized, the ratio of principle curvatures of the pixel data surrounding the keypoint location is calculated. The DoG operation will find many points along edges, some of which are in very "shallow" regions of that curve. Points such as these have small principle curvatures in the direction of the edge, and large principle curvature perpendicular to it. The principal curvature can be 
determined through the calculation of a Hessian matrix centered around the keypoint location:

$$
H=\left[\begin{array}{ll}
D_{x x} & D_{x y} \\
D_{x y} & D_{y y}
\end{array}\right]
$$

The eigenvalues of the matrix in (5) are proportional to the principal curvatures of the area surrounding the keypoint. If the larger eigenvalue is referred to as $E_{1}$ and the smaller referred to as $\mathrm{E}_{2}$, the following relationships hold true:

$$
\begin{aligned}
& \operatorname{Trace}(H)=D_{x x}+D_{y y}=E_{1}+E_{2} \\
& \operatorname{Det}(H)=D_{x x} D_{y y}-\left(D_{x y}\right)^{2}=E_{1} E_{2}
\end{aligned}
$$

If the ratio of the smaller eigenvalue to the large one is defined as $r$, so that $E_{1}=r E_{2}$, then the follow is also true:

$$
\frac{\operatorname{Trace}(H)^{2}}{\operatorname{Det}(H)}=\frac{\left(E_{1}+E_{2}\right)^{2}}{E_{1} E_{2}}=\frac{(r+1)^{2}}{r}
$$

Therefore, to eliminate poorly localized keypoints along edges, a threshold $\mathrm{r}$ can be applied that requires the Hessian matrix of a keypoint (5) to satisfy the following:

$$
\frac{\operatorname{Trace}(H)^{2}}{\operatorname{Det}(H)}<\frac{(r+1)^{2}}{r}
$$

\section{Orientation Assignment}

To create an accurate description of the keypoints for matching and to achieve invariance to rotational changes in an image, the orientation of a keypoint must be determined accurately, and in a way that produces consistent results. 
First, a Gaussian smoothed image L (from 1) at the scale of the SIFT keypoint is selected so that the orientation is determined in a scale-invariant space. The orientation and gradient magnitude are determined for each of the image samples $\mathrm{L}(\mathrm{x}, \mathrm{y})$ at this scale:

$$
\begin{gathered}
m(x, y)=\sqrt{[L(x+1, y)-L(x-1, y)]^{2}+[L(x, y+1)-L(x, y-1)]^{2}} \\
\theta(x, y)=\tan ^{-1}\left[\frac{L(x, y+1)-L(x, y-1)}{L(x+1, y)-L(x-1, y)}\right]
\end{gathered}
$$

Once these orientations and gradient magnitudes are computed, the following occurs:

- A circular Gaussian window with a $\sigma 1.5$ times the size of the scale of the keypoint is applied to the area around the keypoint.

- The points in this area are then weighted by their gradient magnitude.

- The gradient orientations of these local points are entered into an orientation histogram containing 36 bins, and representing the full $360^{\circ}$ range of directions.

- The peaks of the orientation histogram correspond to the strongest orientations of the region. The highest peak is chosen as a possible orientation, as well as any other peaks within $80 \%$ of the value of the highest peak. This results in multiple keypoints being generated at the same scale and location, varying only in orientation.

- The orientation of the keypoint is interpolated from the 3 closest neighboring histogram values to each peak, by fitting all three points to a parabola. 


\section{Keypoint Descriptor}

The orientation histograms described in step 4 are also useful in describing a SIFT point for later matching. The area surrounding the keypoint is divided into a $4 \times 4$ grid. Each region in the grid contains 8 histogram orientation bins, representing the 4 cardinal directions, and the 4 directions bisecting them. This results in a feature vector that is 128 elements long.

\section{Matching}

There are multiple ways of matching keypoint descriptors in order to match keypoints. The simplest method is a comparison of Euclidian distances between descriptors. Descriptors that are closest to each other relative to all other descriptors are matched. 


\section{Overview of Method}

As stated previously, the method in this paper combines the MSER detector and SIFT in order to detect and track planar regions in a scene. The following step by step overview of the method follows the numbering of Figure 15. Refer to different blocks in the diagram while reading through each step. Throughout this overview, Frame 23 of a translational test of scene Outdoor 1 is used as an example.

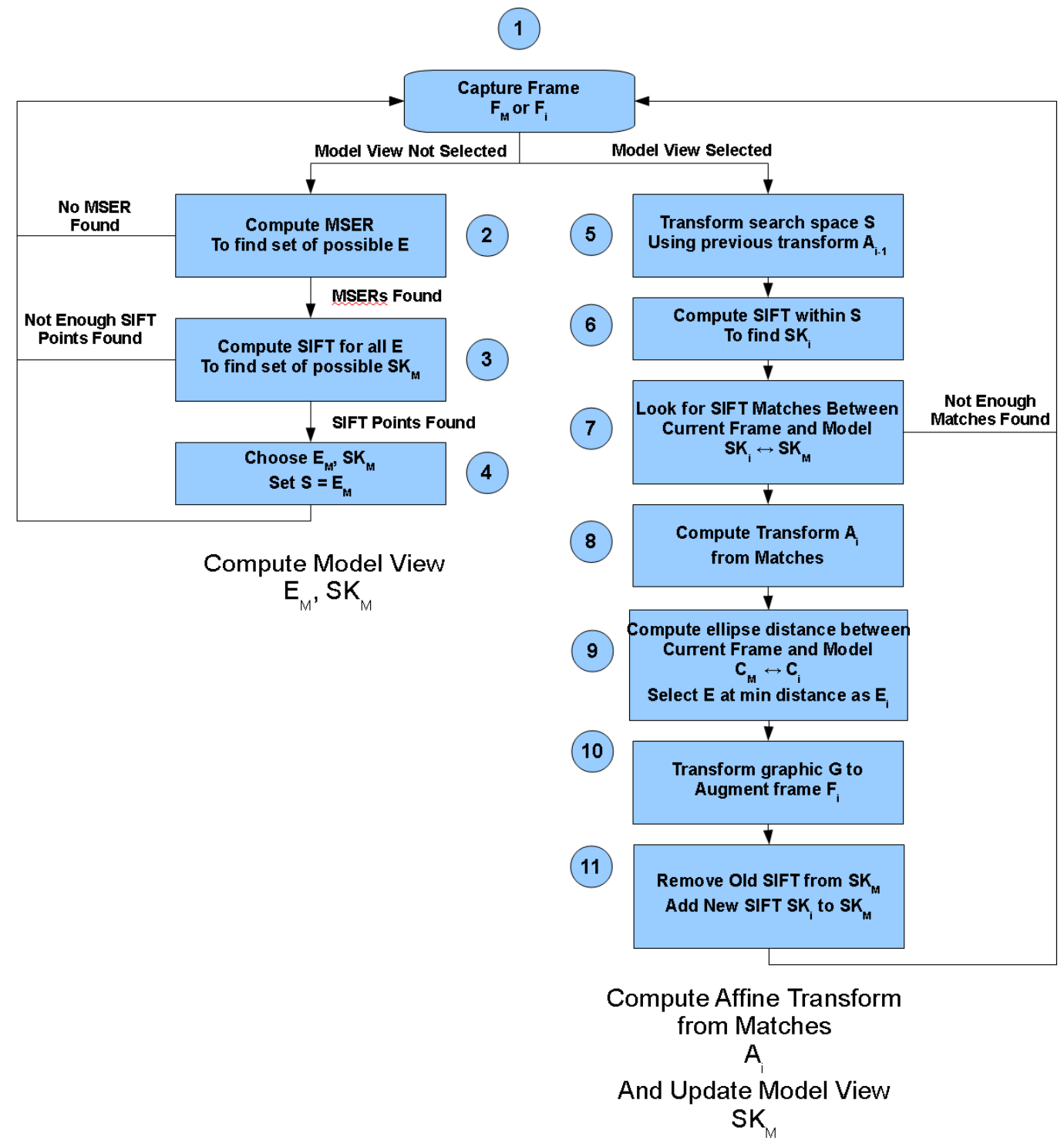

Figure 15: Flowchart of Method 
One thing to note about Figure 15 is that it divides the method into two main sections: computing the model view and computing the affine transform and updating the model view. The first part of computing the model view is only performed once at the beginning of the method.

\section{Compute Model View}

1. The first frame captured and kept is considered to be a potential model view frame $\left(F_{M}\right)$.

2. The MSER is computed and the set of representative ellipses of all MSERs detected is created, as shown in Figure 16.

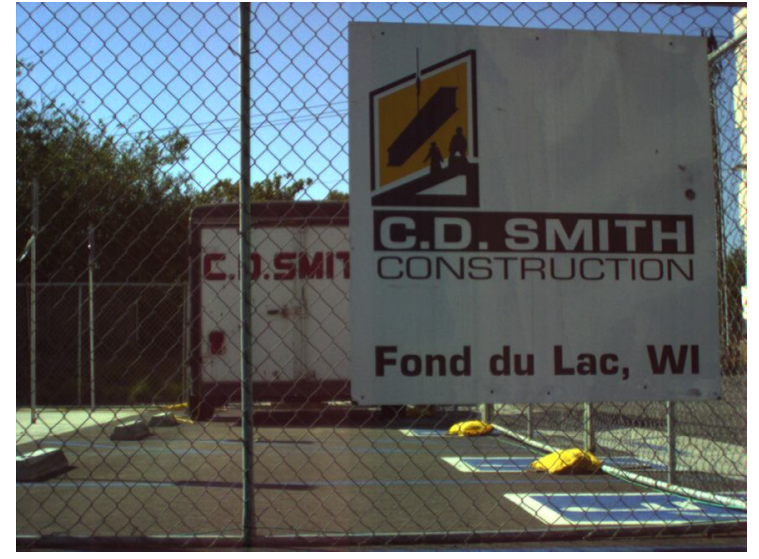

a)

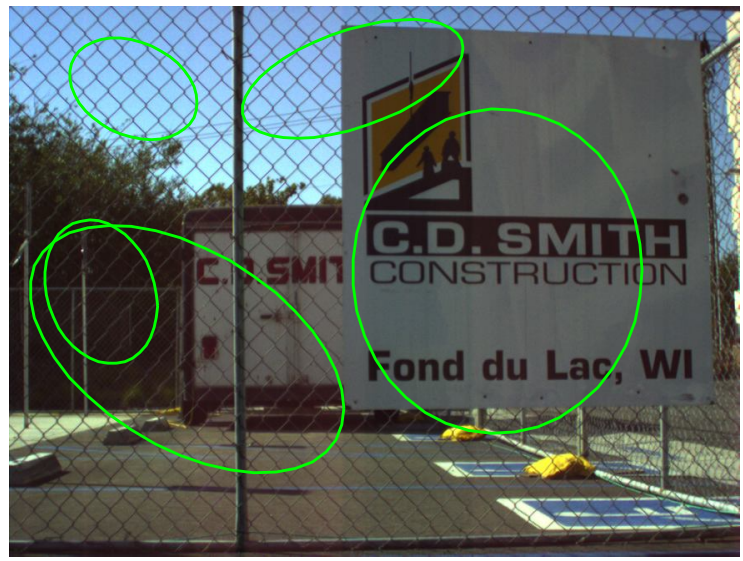

b)

Figure 16: a) Potential Model View Frame, b) Example of Model Frame with Possible Model Ellipses

Displayed

3. Each representative ellipse is examined individually. The region enclosed by a representative ellipse is masked by a binary image of the ellipse (Figure 17a), and the SIFT keypoints are calculated for the region (Figure 17b). 


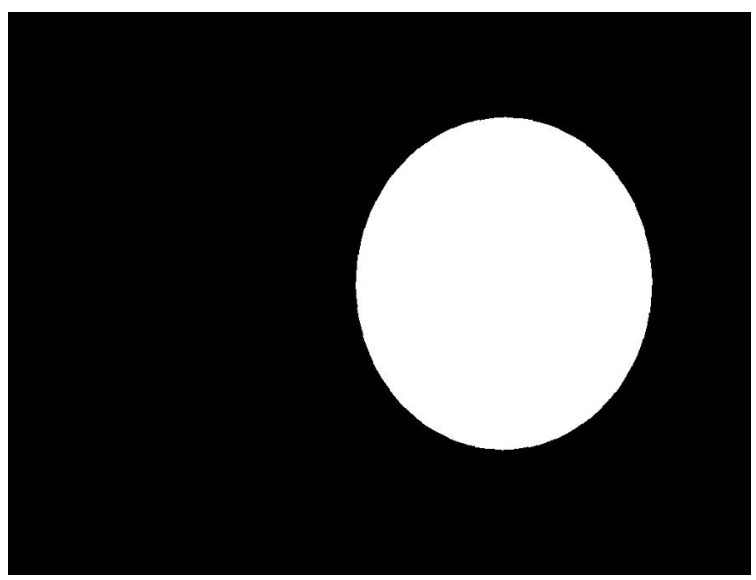

a)

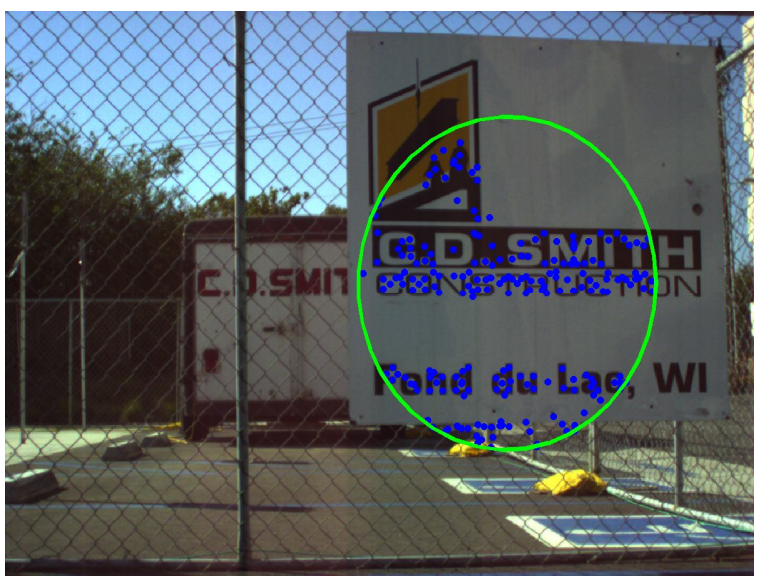

b)

Figure 17: a) Binary Image of Possible Model Ellipse, b) Possible Model Ellipse and Enclosed SIFT keypoints

4. If the number of SIFT keypoints detected is greater than a chosen threshold and the user has selected an area to augment near that ellipse, that region is chosen as the model view, setting $E_{M}$ and $S K_{M}$. (In the case of the example, the ellipse pictured in Figure 17 is chosen as the model ellipse, and the SIFT keypoints pictured are chosen as the model keypoints.)

\section{Compute Affine Transform from Matches and Update Model View}

After the model view frame is captured, the main part of the method is entered.

Each new frame $F_{i}$ that is captured is used to compute an affine transform $A_{i}$ with respect to the model view, if possible.

5. To reduce computation time, the region of the image enclosed by the search space $S$ is masked. $\mathrm{S}$ for the first frame after the model frame is chosen to be the same as the model ellipse $E_{M}$, pictured in Figure $17 \mathrm{a}$. In subsequent frames, $E_{M}$ is transformed by the affine transform to define the current search space (part of step $10)$. 
6. Next, SIFT keypoints are generated in this region forming the current set of keypoints, $S K_{i}$. The current keypoints, $\mathrm{SK}_{23}$, for Frame 23 are shown in Figure $18 \mathrm{~b}$.

7. A search for matching SIFT keypoints between the model view points $\left(S K_{M}\right)$ and the current frame points $\left(S K_{i}\right)$ is performed. $\mathrm{SK}_{\mathrm{M}}$ for the example is shown in Figure 18a.

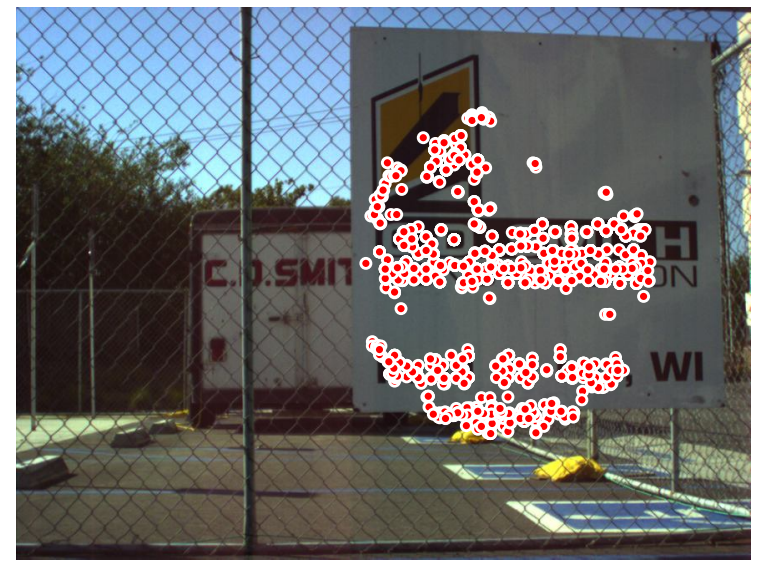

a)

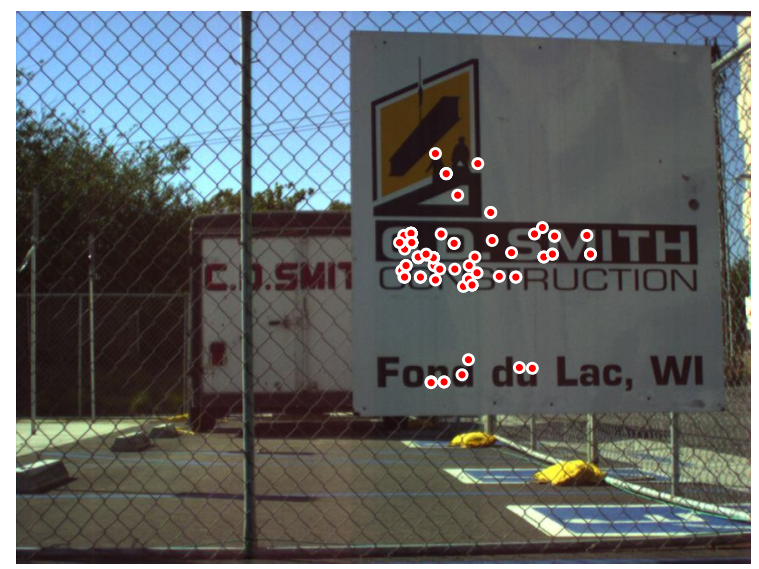

c)

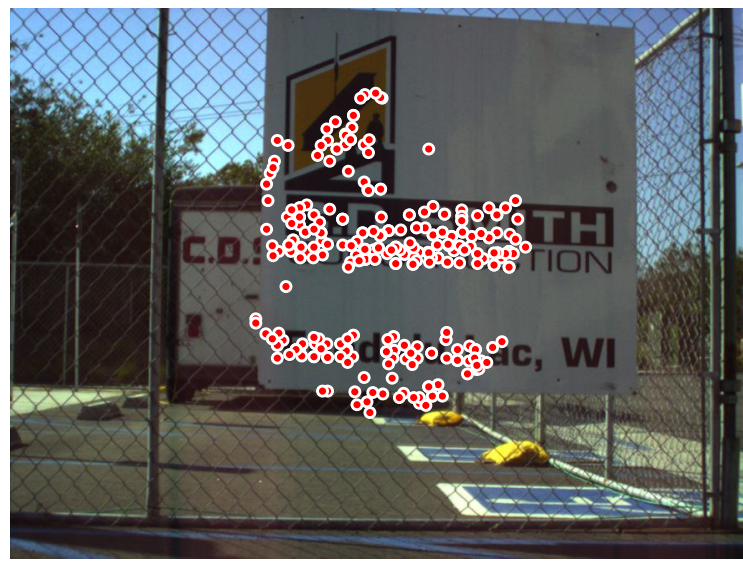

b)

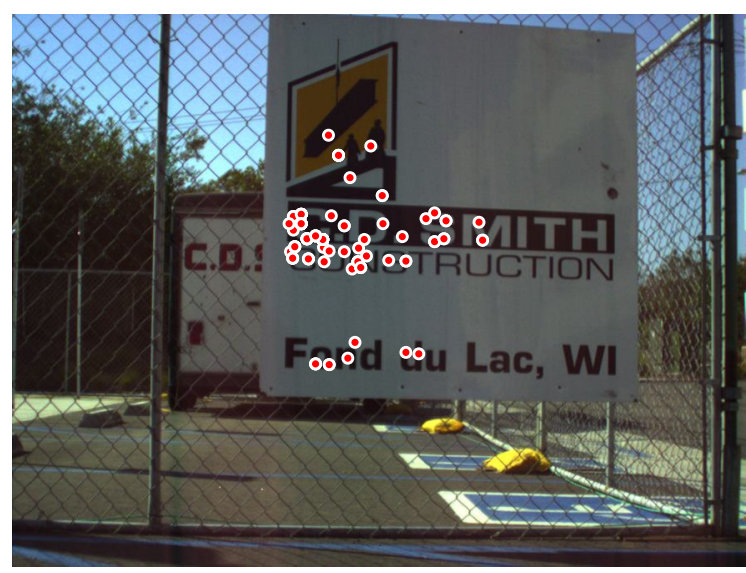

d)

Figure 18: SIFT keypoints in Model Frame and Frame 23 of Example, a) Model View SIFT keypoints, b) Keypoints found in search space of Frame 23, c) Keypoints from model view matched with frame 23, d) keypoints in frame 23 matched with model view 
8. If the three or more point matches required to calculate an affine transform are found, the affine transform $\left(A_{i}\right)$ is computed. It is easily seen that well over 3 keypoint matches are found between the model frame and the current frame 23 in Figure 18c and Figure 18d respectively. All matching points found are used to compute the affine transform.

Recall from affine geometry:

An affine transform allows for geometric translation, scaling, and rotation of points in an image. A simple way of interpreting this is to say that affine transforms map straight lines to straight lines, maintaining distances between these lines. The transform moves a point $(\mathrm{x}, \mathrm{y})$ to a new image space $(\mathrm{u}, \mathrm{v})$ and can be represented as the product of a square matrix for rotation and scaling, summed with a two element translational vector:

$$
\left[\begin{array}{l}
u \\
v
\end{array}\right]=\left[\begin{array}{ll}
m_{1} & m_{2} \\
m_{3} & m_{4}
\end{array}\right]\left[\begin{array}{l}
x \\
y
\end{array}\right]+\left[\begin{array}{l}
t_{x} \\
t_{y}
\end{array}\right]
$$

This can be re-written as a product of a $3 \times 3$ square matrix, with translation included, thus simplifying the equation to one matrix multiplication:

$$
\left[\begin{array}{c}
u \\
v \\
1
\end{array}\right]=\left[\begin{array}{ccc}
m_{1} & m_{2} & t_{x} \\
m_{3} & m_{3} & t_{y} \\
0 & 0 & 1
\end{array}\right]\left[\begin{array}{l}
x \\
y \\
1
\end{array}\right]
$$

This equation can be re-written to solve for the unknowns if given a set of at least three corresponding data points, uv and xy: 


$$
\begin{aligned}
& {\left[\begin{array}{cccccc}
x_{1} & y_{1} & 0 & 0 & 1 & 0 \\
0 & 0 & x_{1} & y_{1} & 0 & 1 \\
x_{2} & y_{2} & 0 & 0 & 1 & 0 \\
0 & 0 & x_{2} & y_{2} & 0 & 1 \\
& & \cdots & & & \\
& & \cdots & & & \\
x_{n} & y_{n} & 0 & 0 & 1 & 0 \\
0 & 0 & x_{n} & y_{n} & 0 & 1
\end{array}\right]\left[\begin{array}{c}
m_{1} \\
m_{2} \\
m_{3} \\
m_{4} \\
t_{x} \\
t_{y}
\end{array}\right]=\left[\begin{array}{c}
u_{1} \\
v_{1} \\
u_{2} \\
v_{2} \\
\vdots \\
u_{n} \\
v_{n}
\end{array}\right]} \\
& A x=b
\end{aligned}
$$

Equation (3) is simply a linear equation, and can be solved by performing a matrix division $\mathrm{x}=\mathrm{b} / \mathrm{A}$ in order to solve for the unknowns. This provides the affine transform required to augment a region of a scene as it undergoes affine transformations.

9. The MSER is computed within the search space $\mathrm{S}$ to find a set of possible ellipses. The center point of the model ellipse $\mathrm{C}_{M}$ is transformed to the current frame using the affine transform $A_{i}$ to find the predicted center point $\hat{C}$. A sum of squared differences approach is taken to compare the center point $\mathrm{C}$ of one of the ellipses found in the current frame to the predicted center point $\hat{C}$. The closest match is considered to be the current ellipse $\mathrm{E}_{\mathrm{i}}$. The distance calculated is used as a criterion for the accuracy of the transform $A_{i}$. 


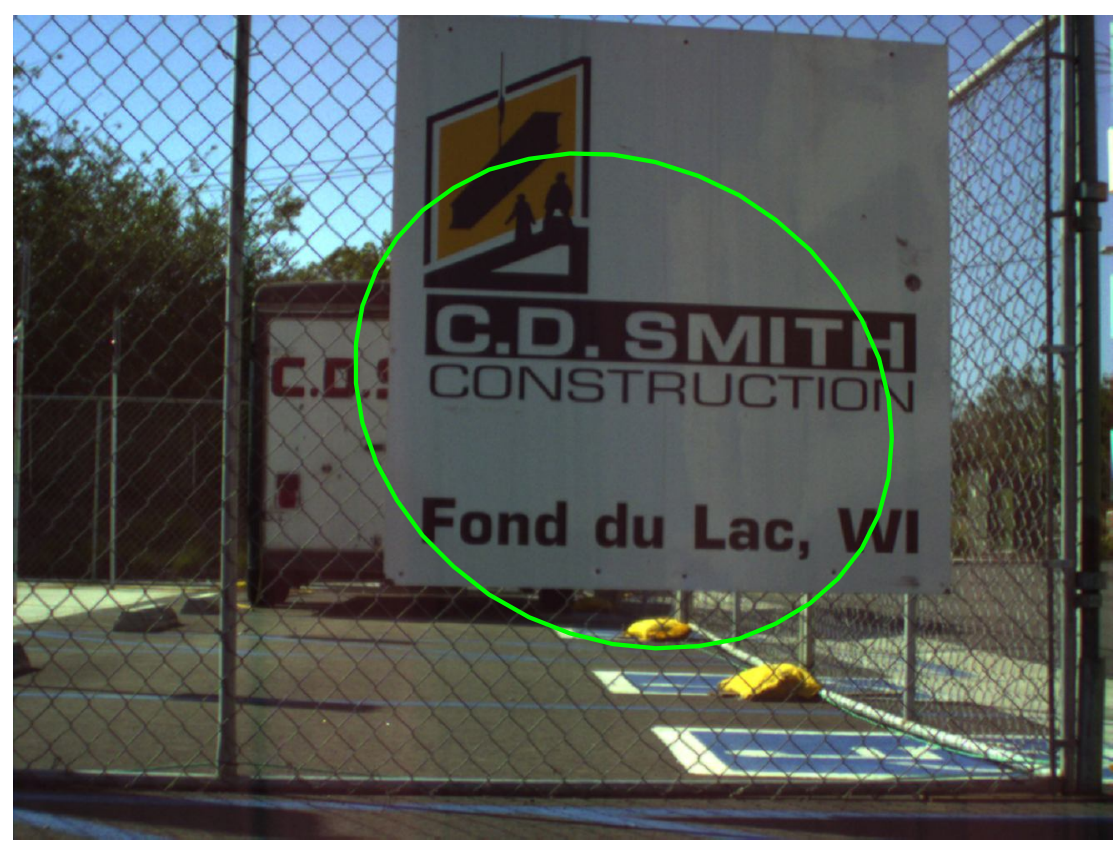

Figure 19: Current Ellipse $E_{23}$ of Frame 23 with closest center point to predicted ellipse

10. The transform is applied to the graphic $G$ that is to be augmented onto the scene, as well as the search space $S$ shown in Figure 20a and Figure 20b respectively. Finally, the transformed $G$ is placed at the center point of the representative ellipse $E_{i}$ which augments the $2 \mathrm{D}$ surface represented by the ellipse. 


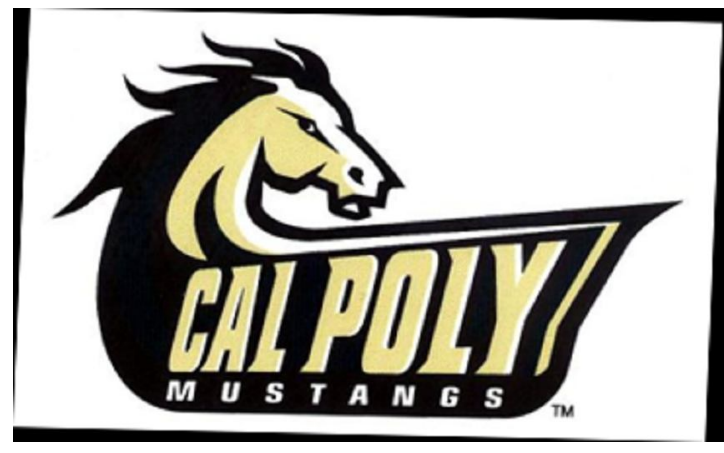

a)

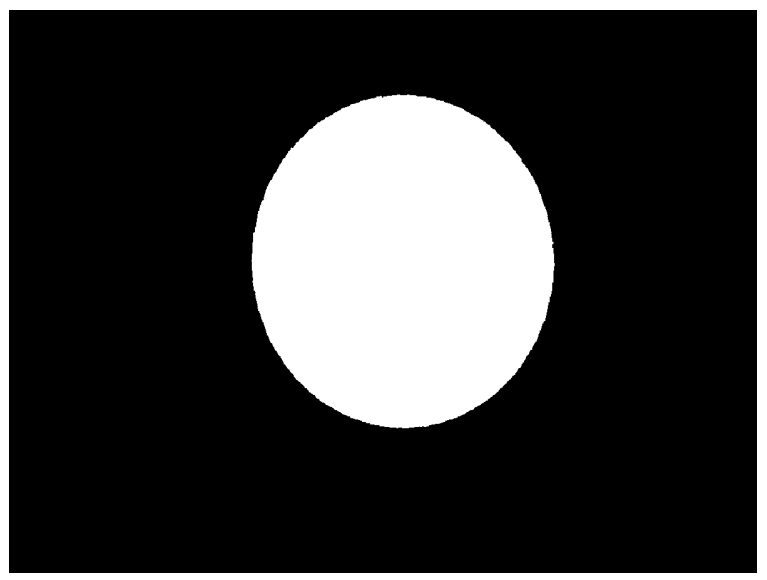

b)

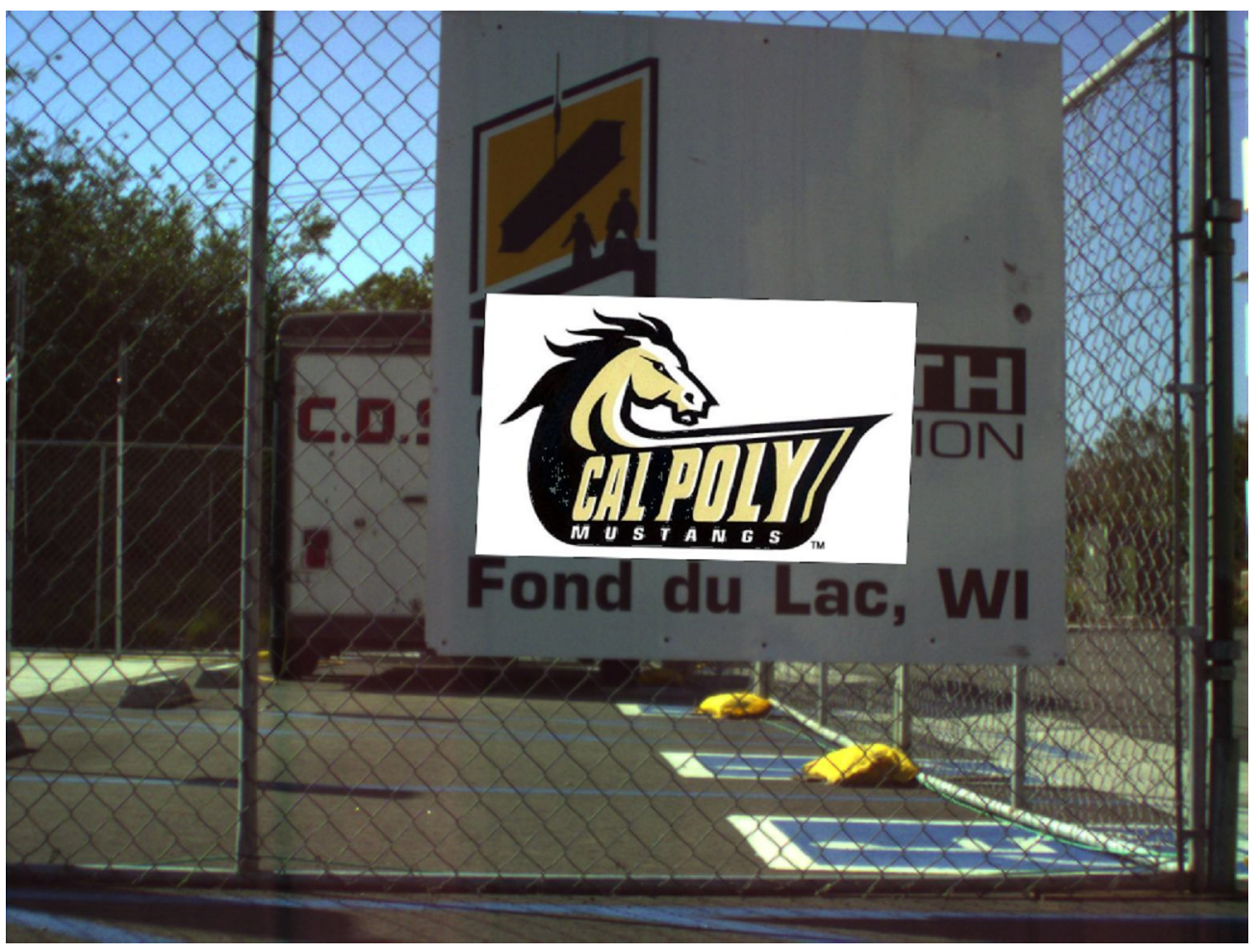

c)

Figure 20: Images Transformed by $A_{i}$, a) Transformed Graphic used for Augmentation, b) Transformed Search Space used to search for keypoints and ellipses in the next frame, c) Augmented version of Example Frame 23

11. In order to take into account new SIFT points detected in the current frame $\mathrm{SK}_{\mathrm{i}}$,

$\mathrm{SK}_{\mathrm{i}}$ points are added to the model view $\mathrm{SK}_{\mathrm{M}}$. Once a certain threshold of

keypoints is reached, older keypoints are removed to make room for newer 
keypoints. By doing this, the method is very robust to incremental changes from frame to frame, because the most recent frames SIFT points are weighted as heavily as the original model view SIFT points. A progression of SIFT keypoints being added to the Model View is illustrated in Figure 21 a-d. Figure 21a shows the original Model View keypoints, and subsequent images b-d show additional keypoints added, as well as keypoints removed.

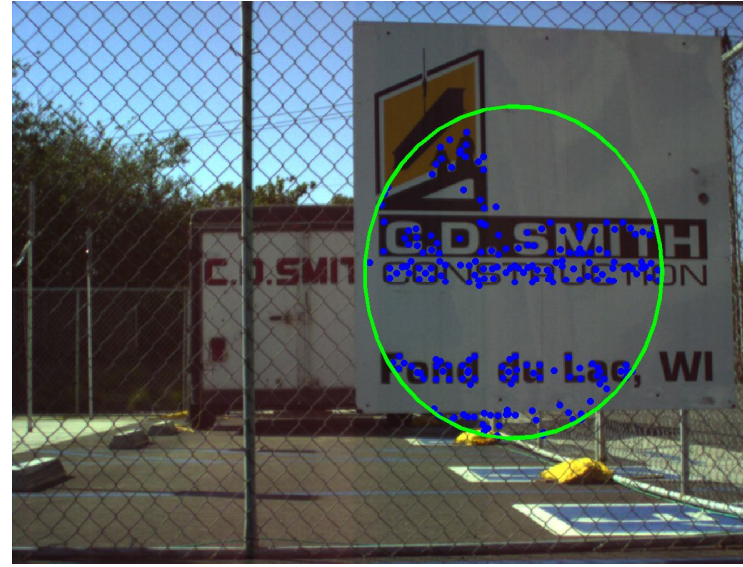

a)

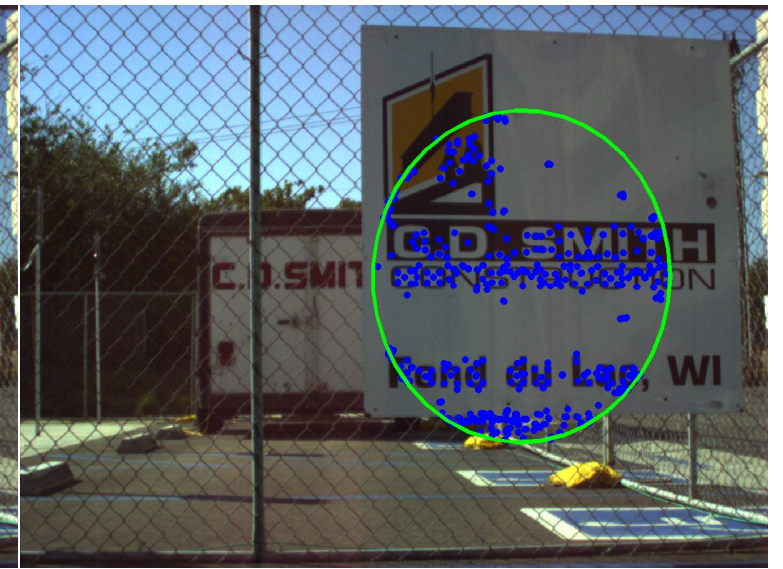

b)

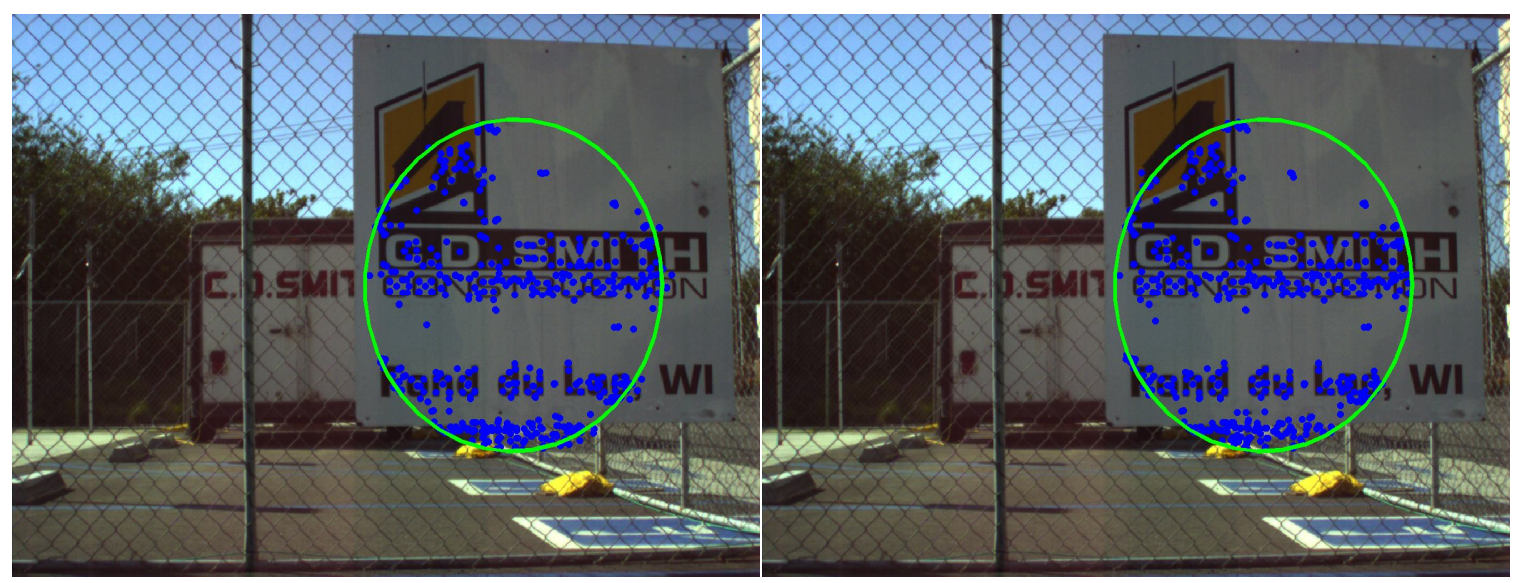

c) d)

Figure 21: Progression of SIFT keypoints added and removed from model view, a) Original Model View, b) Model View Frame 7, c) Model View Frame 15, d) Model View Frame 23 


\section{Chapter 4: Experimental Setup and Results of Testing}

\section{Experimental Setup}

To test the performance of the system several criteria will be measured while varying the translation, rotation, and scaling of objects or the camera in the environment. The surface type being augmented will be varied with the scene, and the lighting conditions will vary naturally with each scene. These different conditions are outlined in Table 4 and

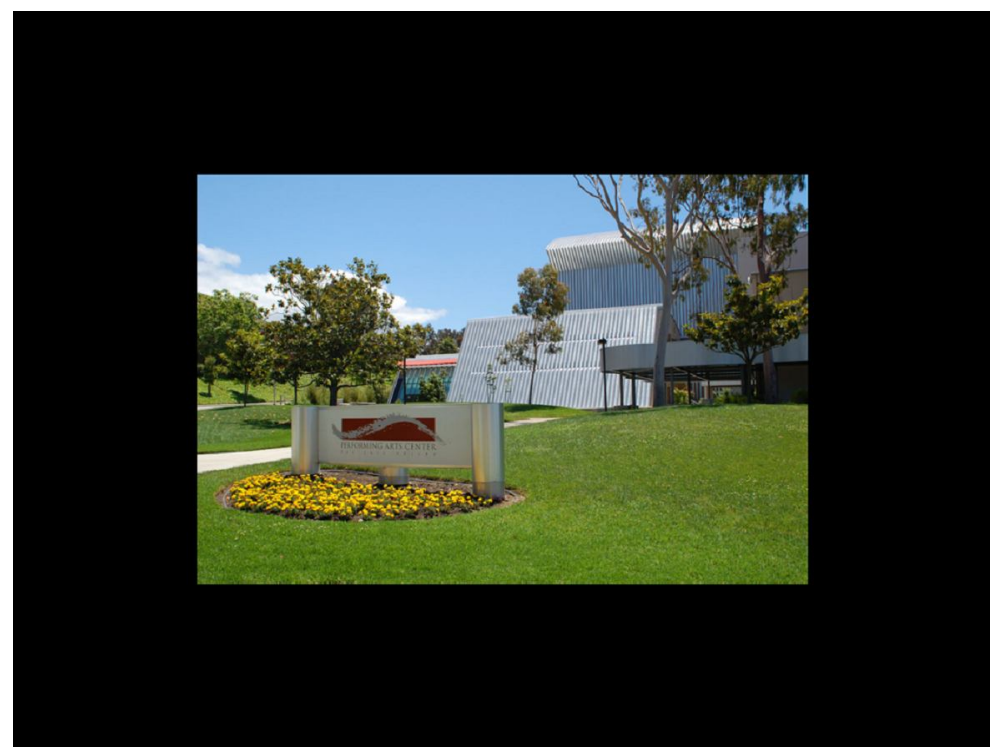

Figure 23: Image of Ideal Scene

Table 5.

Table 4: Testing Variables

\begin{tabular}{|c|l|c|c|}
\hline $\begin{array}{c}\text { Type of } \\
\text { Invariant } \\
\text { / Issues }\end{array}$ & Scenes & Variable & $\begin{array}{c}\# \text { of } \\
\text { Test } \\
\mathbf{s}\end{array}$ \\
\hline $\begin{array}{c}\text { Translatio } \\
\mathrm{n}\end{array}$ & 6 Scenes, Listed in & $\begin{array}{c}\text { None } \\
\text { (except } \\
\text { for scene } \\
\text { change) }\end{array}$ & 6 \\
\hline
\end{tabular}




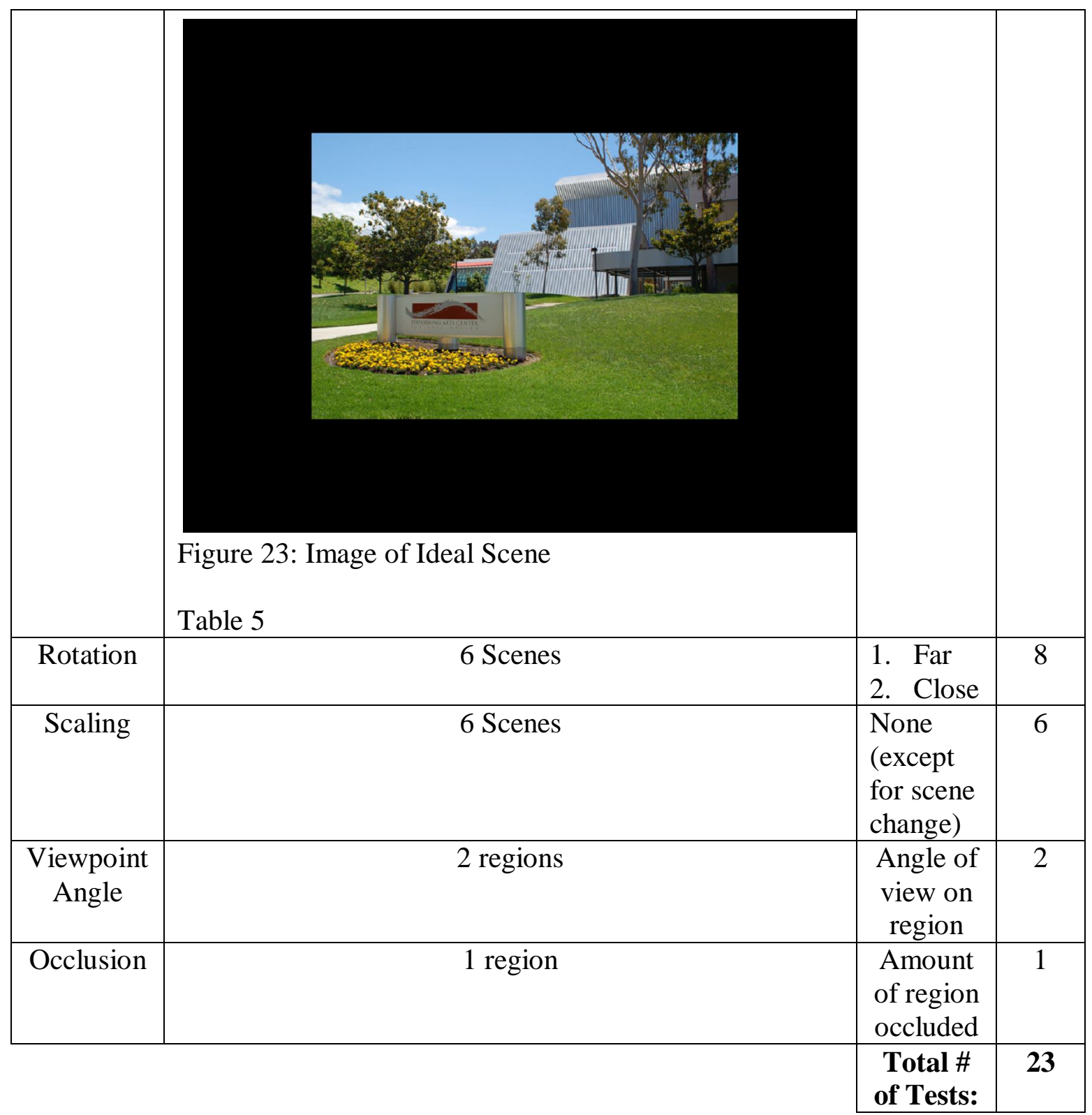

The criteria to be measured to access the performance of the method are:

1. Normalized Cross-Correlation Score (between regions)

A discrete normalized cross-correlation score (ranging from -1 to 1 ) is calculated between the region $R_{i}$ in the current frame and the region $R_{i-1}$ in the previous frame. A non-normalized cross-correlation score is sensitive to changes in 
lighting, which is why the normalized version is used. This score is a good indicator of the accuracy in finding new regions.

2. Prediction Error (center point distance comparison)

When a prediction is made for a region's location in a new frame $F_{i}$, the accuracy of the prediction is measured by transforming the center point $\mathrm{C}_{\mathrm{i}-1}$ of the previous region $R_{i-1}$ to the new region $R_{i}$. The predicted location of the center point $C_{i-1}$ is compared to the actual center point $\mathrm{C}_{\mathrm{i}}$ by computing the distance in pixels.

\section{Number of SIFT Keypoint Matches}

Every frame augmented requires at least 3 SIFT keypoint matches with the model view in order to compute the current affine transform $A_{i}$. The number of matches found is a good indicator of the stability of the system; if the number of matches is close to 3 the system is close to losing stability. 


\section{Description of Scenes}

The following section provides an overview of the scenes used for testing.

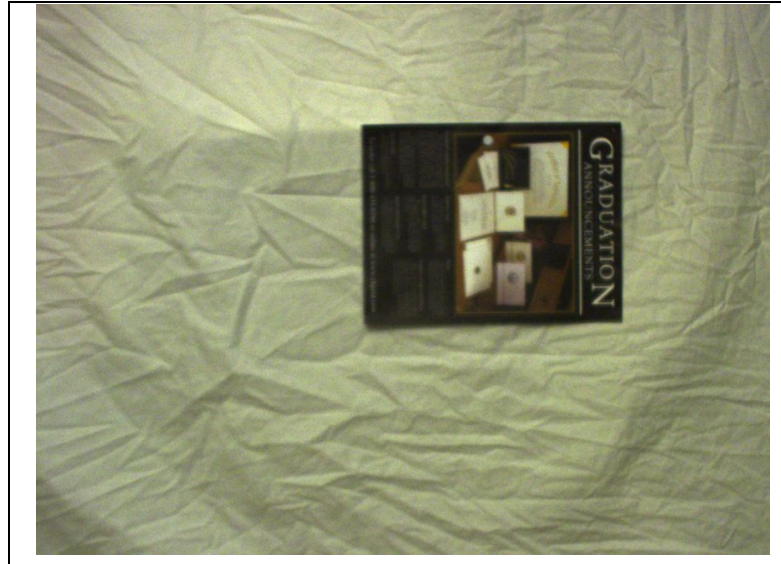

a) Indoor 1

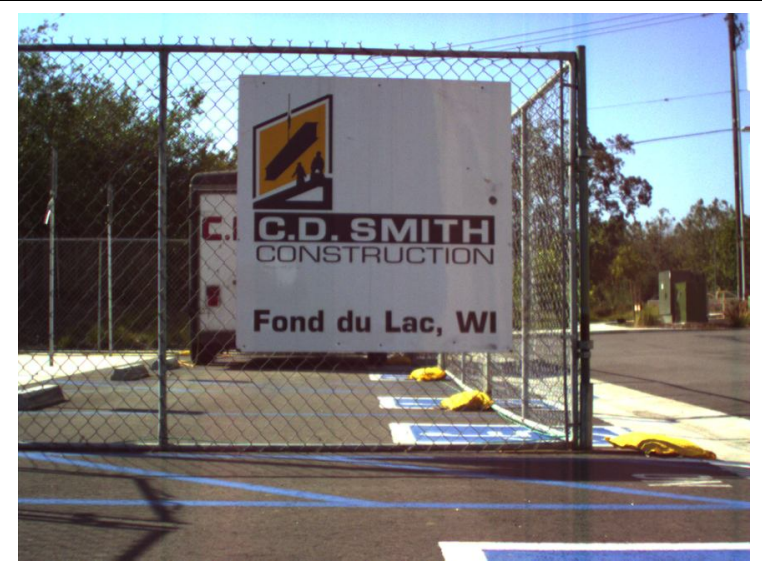

c) Outdoor 1

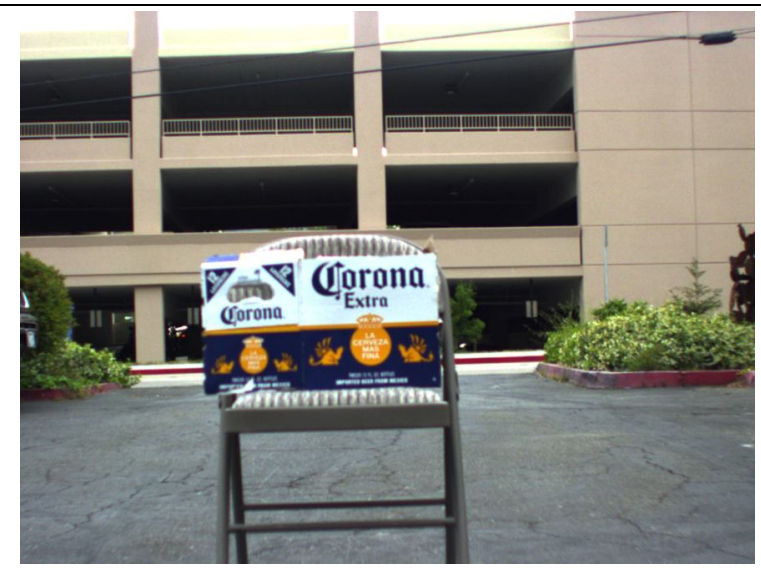

e) Outdoor 3

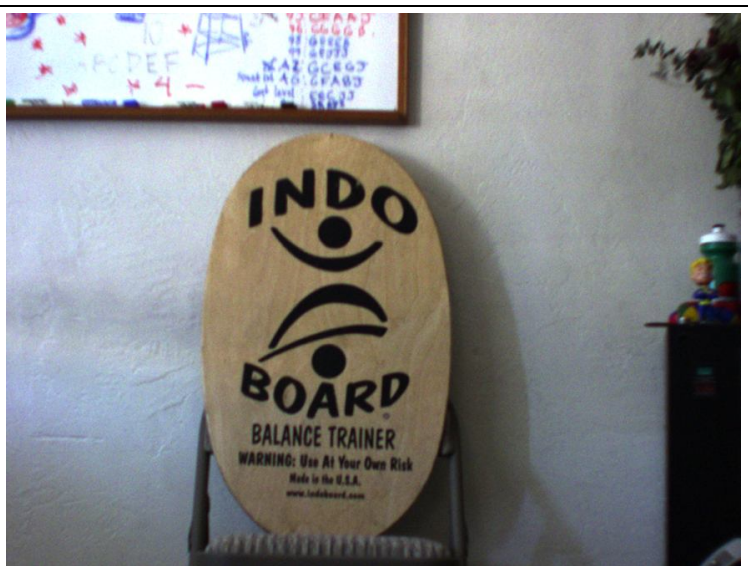

b) Indoor 2

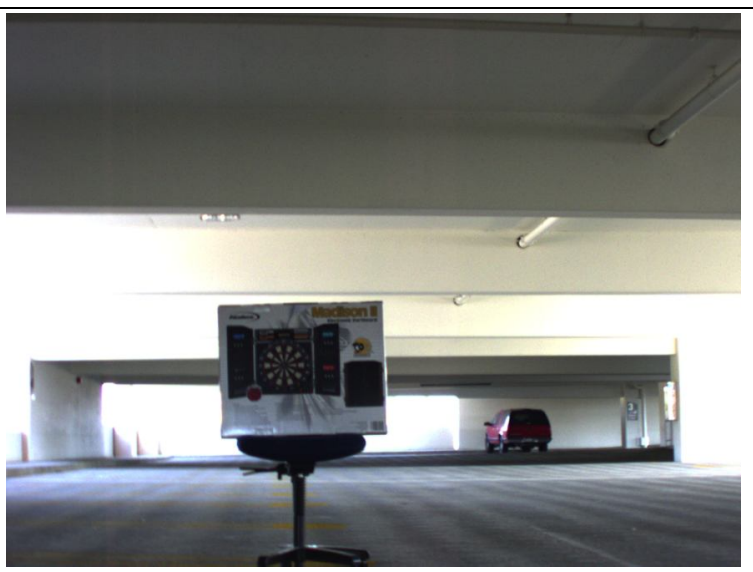

d) Outdoor 2

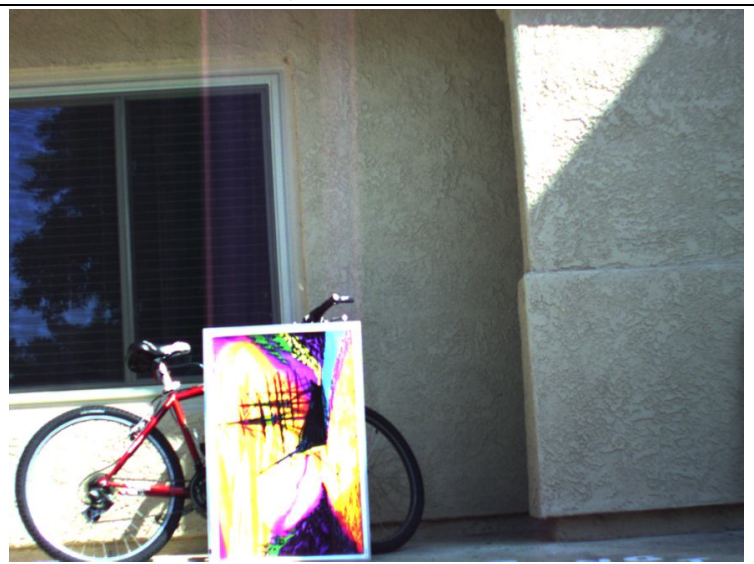

f) Outdoor 4

Figure 22: Images of Real World Scenes (6 total), a) Indoor 1, b) Indoor 2, c) Outdoor 1, d) Outdoor 2, e) Outdoor 3, f) Outdoor 4 


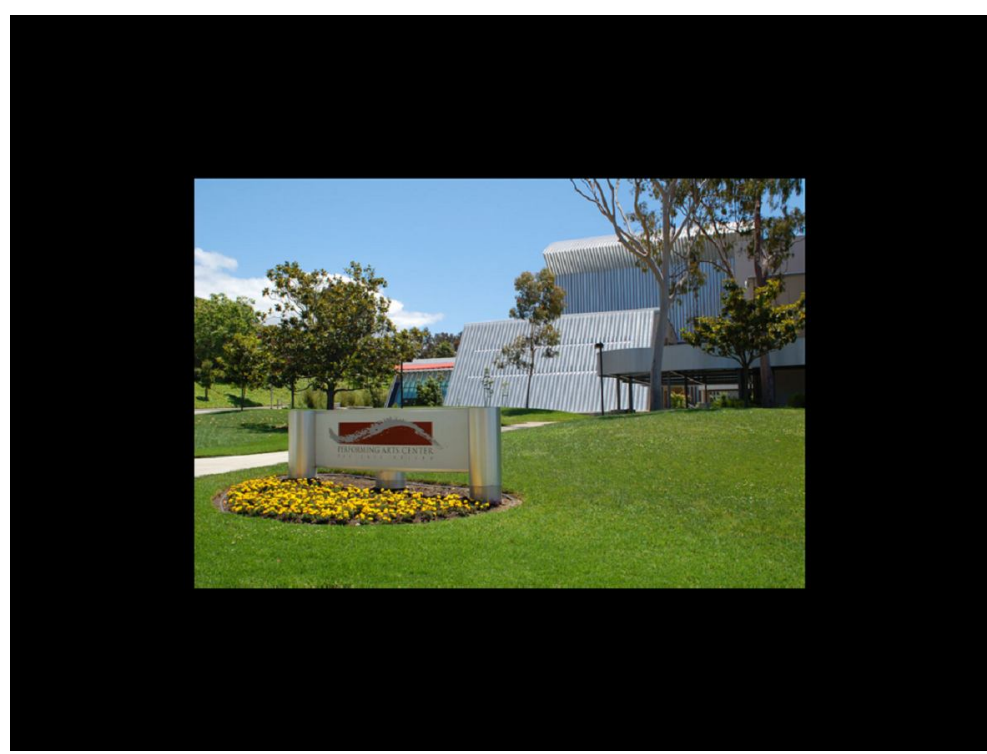

Figure 23: Image of Ideal Scene

Table 5: Testing Scenes

\begin{tabular}{|c|c|c|c|c|c|}
\hline Scene & $\begin{array}{l}\text { Related } \\
\text { Figure }\end{array}$ & Lighting & Distance & $\begin{array}{l}\text { Surface } \\
\text { Type }\end{array}$ & $\begin{array}{c}\text { Texture of } \\
\text { Surrounding } \\
\text { Environment }\end{array}$ \\
\hline Ideal & Figure 23 & Constant & $\mathrm{NA}$ & $\begin{array}{l}1024 \times 681 \\
\text { resolution }\end{array}$ & $\begin{array}{c}\text { Zero (all } \\
\text { black) }\end{array}$ \\
\hline Indoor 1 & Figure $22 \mathrm{a}$ & $\begin{array}{l}\text { Uniform, } \\
\text { low }\end{array}$ & Close (5ft) & $\begin{array}{c}8-1 / 2 \times 11 \\
\text { Sheet of } \\
\text { Paper }\end{array}$ & Low \\
\hline Indoor 2 & Figure $22 b$ & $\begin{array}{c}\text { Sparse, } \\
\text { Ambient } \\
\text { light }\end{array}$ & Close (9ft) & $\begin{array}{l}3 \mathrm{ft} \times 1.5 \mathrm{ft} \\
\text { Board }\end{array}$ & Low \\
\hline $\begin{array}{c}\text { Outdoor } \\
1\end{array}$ & Figure 22c & $\begin{array}{c}\text { Gradient } \\
\text { from left, } \\
\text { Direct } \\
\text { sunlight }\end{array}$ & $\begin{array}{c}\text { Midrange }(15 \mathrm{ft}) \\
- \text { Far }(25 \mathrm{ft})\end{array}$ & $\begin{array}{c}3 \mathrm{ft} \times 3 \mathrm{ft} \\
\text { Sign }\end{array}$ & High \\
\hline $\begin{array}{c}\text { Outdoor } \\
2\end{array}$ & Figure $22 \mathrm{~d}$ & $\begin{array}{c}\text { Uniform, } \\
\text { Direct } \\
\text { sunlight } \\
\end{array}$ & Far (> 50ft) & $\begin{array}{l}2 \mathrm{ft} \times 1.5 \mathrm{ft} \\
\text { Box }\end{array}$ & Low \\
\hline $\begin{array}{c}\text { Outdoor } \\
3\end{array}$ & Figure $22 \mathrm{e}$ & $\begin{array}{l}\text { Uniform, } \\
\text { Indirect } \\
\text { sunlight } \\
\end{array}$ & $\begin{array}{l}\text { Varied (10ft - } \\
50 \mathrm{ft})\end{array}$ & $\begin{array}{c}2 \mathrm{ft} \times 1 \mathrm{ft} \\
\text { Box }\end{array}$ & High \\
\hline $\begin{array}{c}\text { Outdoor } \\
4\end{array}$ & Figure 22f & & $\begin{array}{l}\text { Varied (10ft - } \\
50 \mathrm{ft})\end{array}$ & $\begin{array}{l}3 \mathrm{ft} \times 1.5 \mathrm{ft} \\
\text { Poster }\end{array}$ & High \\
\hline
\end{tabular}


Scene: Indoor 1 (Figure 22a)

Low light, low blur, low texture environment

The first scene is indoors, and the environment is controlled. The lighting is behind the camera, creating even illumination of the entire scene. The texture being augmented is set on top of a white sheet to remove all background texture. Even illumination and no background texture simplify the environment, and enhances the ability of the algorithm to perform well.

Scene: Indoor 2 (Figure 22b)

Low light, medium blur, low texture environment

The second scene is also indoors, but under less ideal conditions. The lighting is low, and from ambient outdoor light through the windows, and the environment is littered with many textured objects.

Scene: Outdoor 1 (Figure 22c)

Good light, low blur, high texture environment

This scene is well illuminated, with little blur, making for an almost ideal case for an outdoor scene. The region being augmented has a moderate amount of SIFT keypoint matches, which proves to be adequate for good augmentation.

Scene: Outdoor 2 (Figure 22d)

Inconsistent light, high blur, low texture environment 
This scene has many of the worst features present in any of the scenes, including poor and inconsistent lighting, blur, and a low number of SIFT keypoints on the surface being augmented.

Scene: Outdoor 3 (Figure 22e)

Saturated light, medium blur, high texture environment

This scene has lighting that appear very good at first glance, but is actually somewhat saturated, which affects the SIFT's ability to consistently detect the same keypoints.

Scene: Outdoor 4 (Figure 22f)

Saturated light, medium blur, high texture environment

This scene has very high illumination, and many regions high in SIFT keypoints surrounding the region of interest, causing some keypoint to be found outside of the augmented region. However, this does not significantly affect the ability of the method to augment the region. 


\section{Translation Setup}

To test the system's ability to handle translational changes the camera is moved in the $\mathrm{x}$-direction, relative to the region being augmented, $\mathrm{R}_{\mathrm{i}}$. The distance $\mathrm{D}$ is varied in each scene (distances noted in Figure 24), varying the translational distance Tx directly (Figure 24). The translational distance traveled during each test is equal to two times the center point distance listed in Table 6 , or the sum of the translational distances noted in Figure 24 (T_start + T_end). In short:

Translated distance $=2 *$ center point distance $=2 * \mathrm{~T} \_$start $=2 * \mathrm{~T} \_$end

Table 6: Translation Test Geometric Description

\begin{tabular}{|c|c|c|}
\hline Scene & Distance to Object (ft) & Center Point Distance (in) \\
\hline Indoor 1 & 5 & 7 \\
\hline Indoor 2 & 6 & 9 \\
\hline Outdoor 1 & 15 & 42 \\
\hline Outdoor 2 & 12 & 37 \\
\hline Outdoor 3 & 12 & 37 \\
\hline Outdoor 4 & 12 & 37 \\
\hline
\end{tabular}

Note: Ideal Scene not listed 

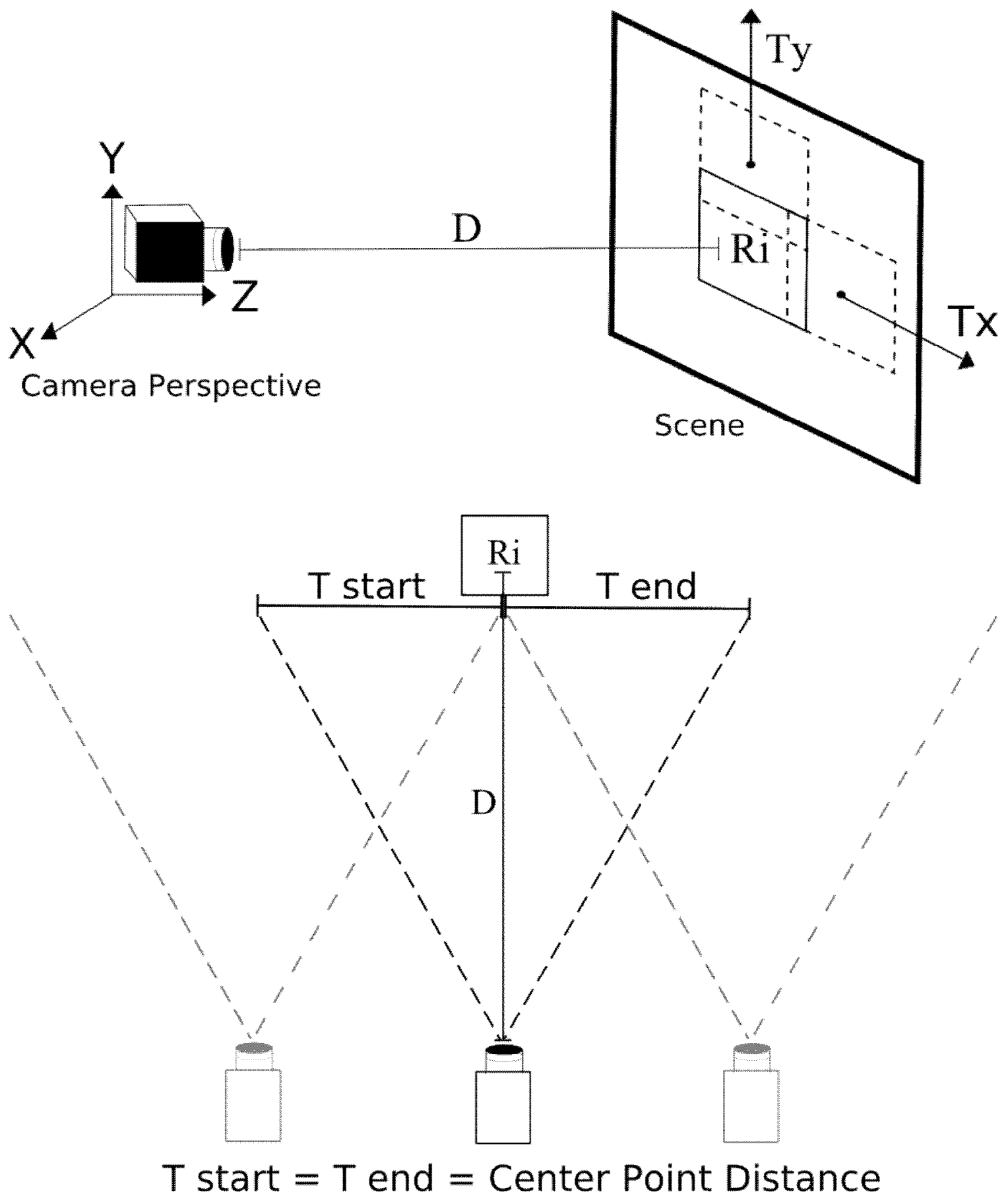

Figure 24: Translation Test Conceptual Diagram 


\section{Rotation Setup}

To test the systems invariance to rotational changes the camera was rotated about its z-axis. The camera sequences were taken so that the z-axis of the camera (centered inside the camera lens, was as close to aligned with the center point of the region as possible. It is evident in the sequences that there is some translation about the center point of the region due to misalignment of the camera and the region.

Table 7: Rotation Test Geometric Description

\begin{tabular}{|l|c|c|}
\hline Scene & Distance to Object $(\mathbf{f t})$ & Rotational Speed $\left({ }^{\circ}\right.$ per Frame $)$ \\
\hline Indoor 1 & 5 & 5.4 \\
\hline Indoor 2 & 6 & 4.2 \\
\hline Indoor 2 & 12 & 4 \\
\hline Outdoor 1 & 12 & 3.6 \\
\hline Outdoor 2 & 12 & 3.6 \\
\hline Outdoor 3 & 12 & 5.4 \\
\hline Outdoor 4 & 12 & 3.6 \\
\hline
\end{tabular}

Note: Ideal Scene not listed

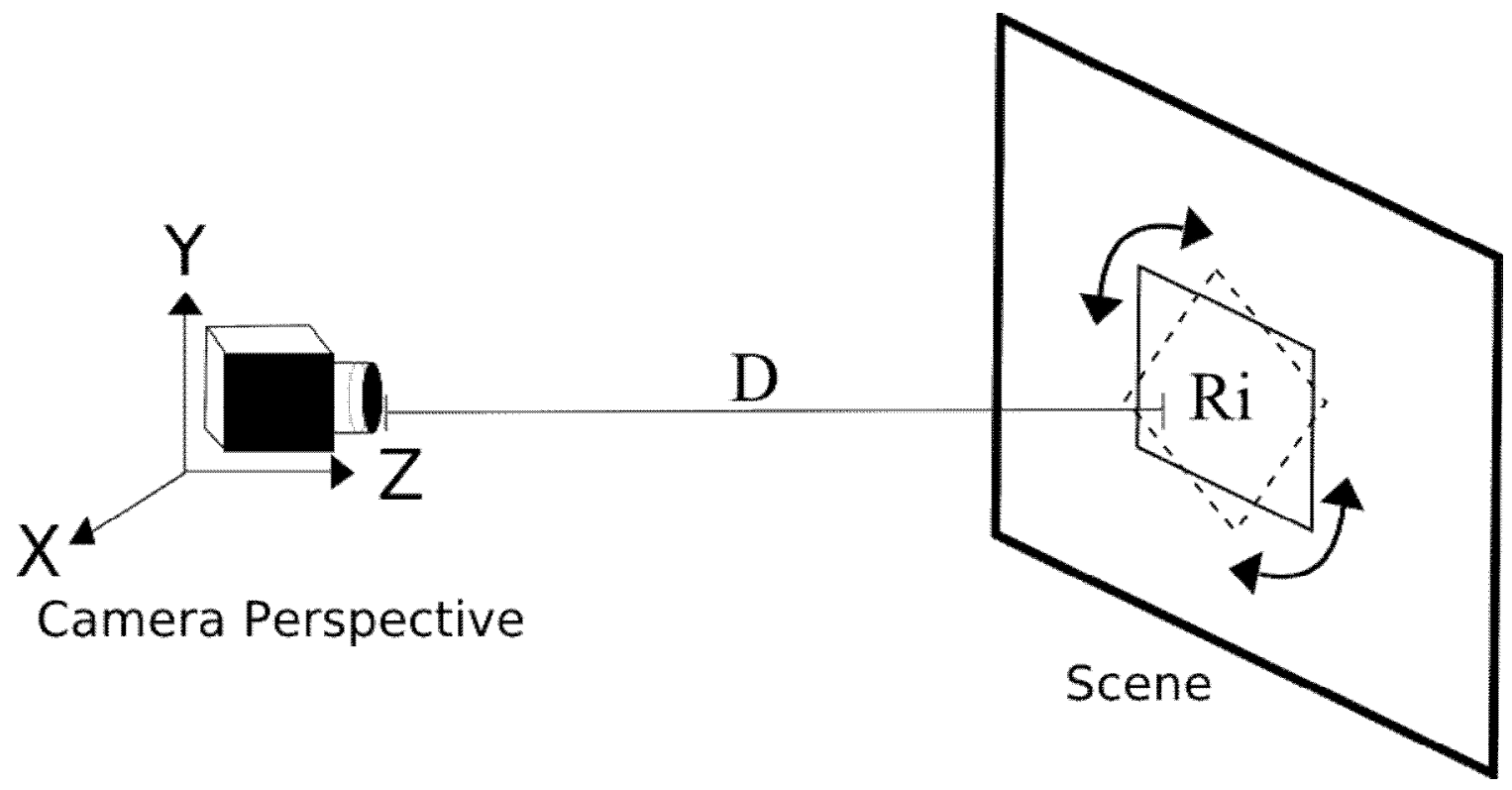

Figure 25: Rotation Test Conceptual Diagram 


\section{Scaling Setup}

To test the system's ability to handle changes in scale, the distance is varied between the camera and the region being augmented, $\mathrm{R}_{\mathrm{i}}$. The change in scale is characterized by a starting distance D_start, and an ending distance D_end, shown in Figure 26. D_start was chosen so that the region fills as much of the camera frame as possible without causing avoidable blur. In other words, the first or last frame of a sequence contains only the region to be augmented.

Table 8: Scaling Test Geometric Description

\begin{tabular}{|l|c|c|}
\hline Scene & Starting Distance (ft) & Ending Distance (ft) \\
\hline Indoor 1 & 2 & 6 \\
\hline Indoor 2 & 3 & 9 \\
\hline Outdoor 1 & 7 & 21 \\
\hline Outdoor 2 & 7 & 21 \\
\hline Outdoor 3 & 3 & 24 \\
\hline Outdoor 4 & 3 & 24 \\
\hline
\end{tabular}

Note: Ideal Scene not listed

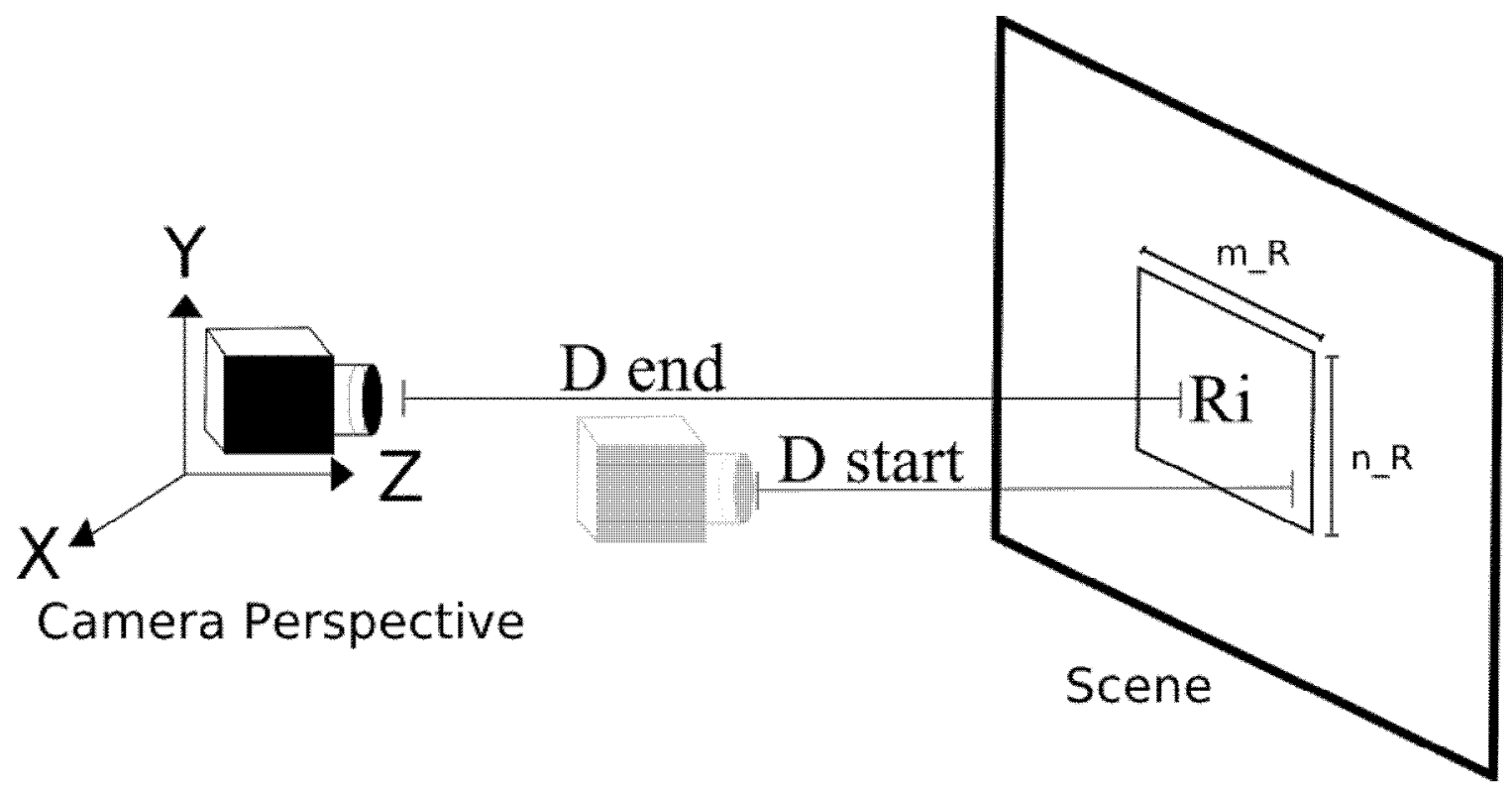

Figure 26: Scaling Test Conceptual Diagram 


\section{Viewpoint Angle Setup}

To test the system's ability to handle viewpoint angle change, a 2D planar surface was rotated out of the plane of the camera lens over a range of angles represented by $\theta$.

When the plane of the camera's lens is parallel to the plane of the region being augmented $\theta=90^{\circ}$, and when the planes are orthogonal $\theta=0^{\circ}$. In both of the tests presented the beginning of the sequence starts with $\theta=90^{\circ}$ and ends with $\theta=0^{\circ}$.

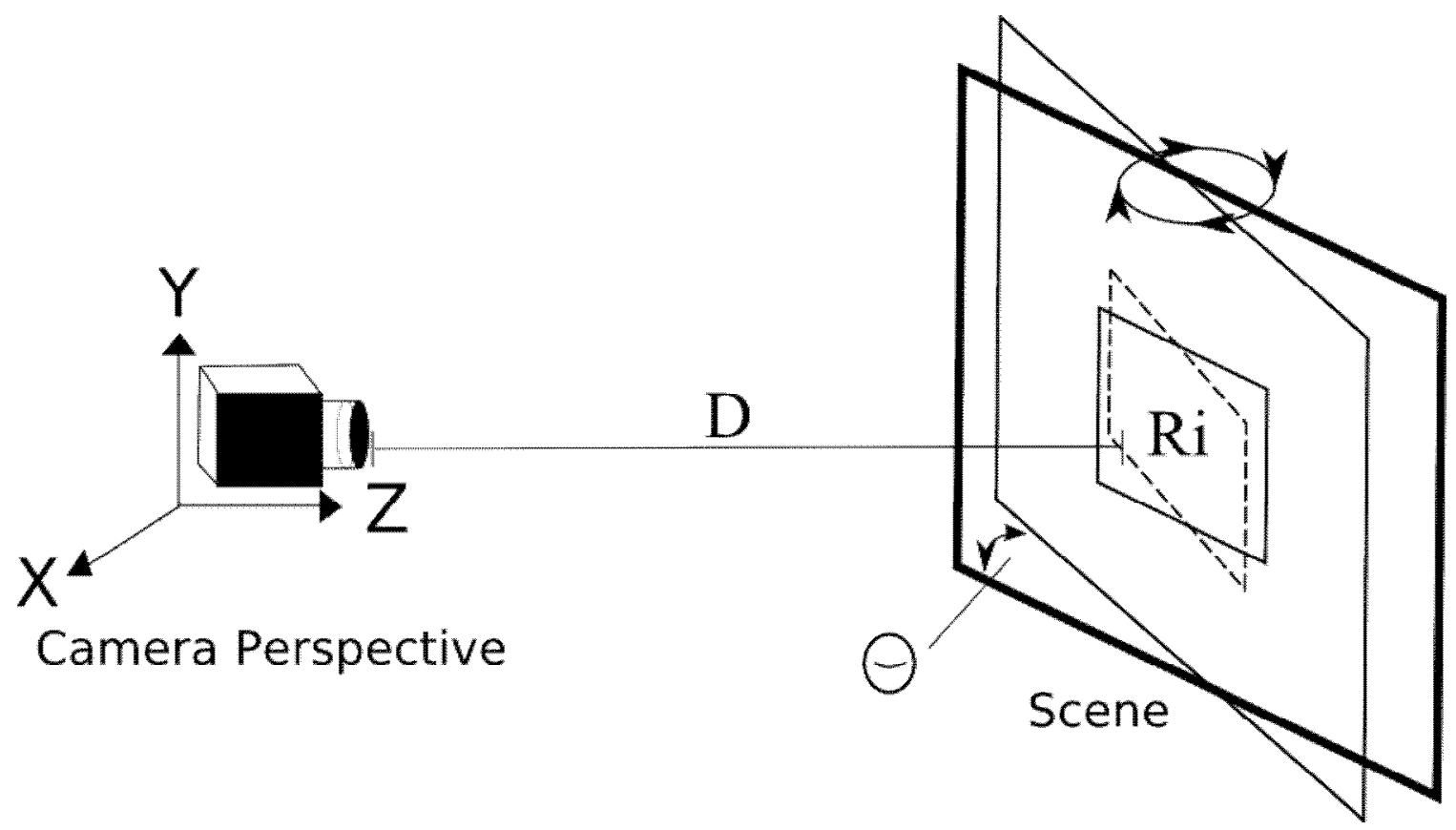

Figure 27: Viewpoint Angle Test Conceptual Diagram 


\section{Testing and Results}

The "Testing and Results" section is concerned with explaining scenes, presenting representative test cases, and analyzing the results. The section is organized into five main sections, based on what variable or issue is being test:

1. Translation Testing and Results

2. Rotation Testing and Results

3. Scale Testing and Results

4. Viewpoint Angle Testing and Results

5. Occlusion Testing and Results

For an explanation for the four testing criteria used for each test see the beginning of Chapter 4. Because each of these five tests has anywhere from one to six scenes included in it, there are a tremendous amount of images, data, and plots generated. To make the analysis of the data more manageable only one representative case is shown, followed by the mean of the data from all scenes included in that test. The images, data, and plots for all other test cases can be found in Appendix A. Each of the five sections follows a similar format:

1. A "Representative Test" is presented, based on the scene Outdoor 1 Tests

a. Images of 4 representative frames of the scene are shown

i. Model View: Model Ellipse and Model SIFT Keypoints

ii. Augmented Frame: Current Ellipse, Current SIFT Points, Augmented Graphic

iii. Search Space (binary image)

b. Plots of 4 different criteria for evaluating the representative scene are shown

i. SIFT Keypoint Matches

ii. Center Point Pixel Distances

iii. Cross Correlation Btwn Current and Previous Frame

iv. Cross Correlation Btwn Current and Model Frame

c. A table of the mean values of the data for the representative scene is shown

i. Mean SIFT Keypoint Matches

ii. Mean Center Point Pixel Distances

iii. Mean Cross Correlation Btwn Current and Previous Frame 
iv. Mean Cross Correlation Btwn Current and Model Frame

2. Plots of the mean values of the data for ALL scenes is shown

a. Mean SIFT Keypoint Matches

b. Mean Center Point Pixel Distances

c. Mean Cross Correlation Btwn Current and Previous Frame

d. Mean Cross Correlation Btwn Current and Model Frame

3. A table of the mean values of the data for ALL scenes is shown

a. Mean SIFT Keypoint Matches

b. Mean Center Point Pixel Distances

c. Mean Cross Correlation Btwn Current and Previous Frame

d. Mean Cross Correlation Btwn Current and Model Frame

4. There is a Discussion of the Results of the section

Notes about the data:

- A '0' value for Center Point Pixel Distances indicates no current ellipse found 
Translation Testing and Results

Representative Translation Test: Indoor $1, \mathrm{D}=\mathbf{5 f t}$

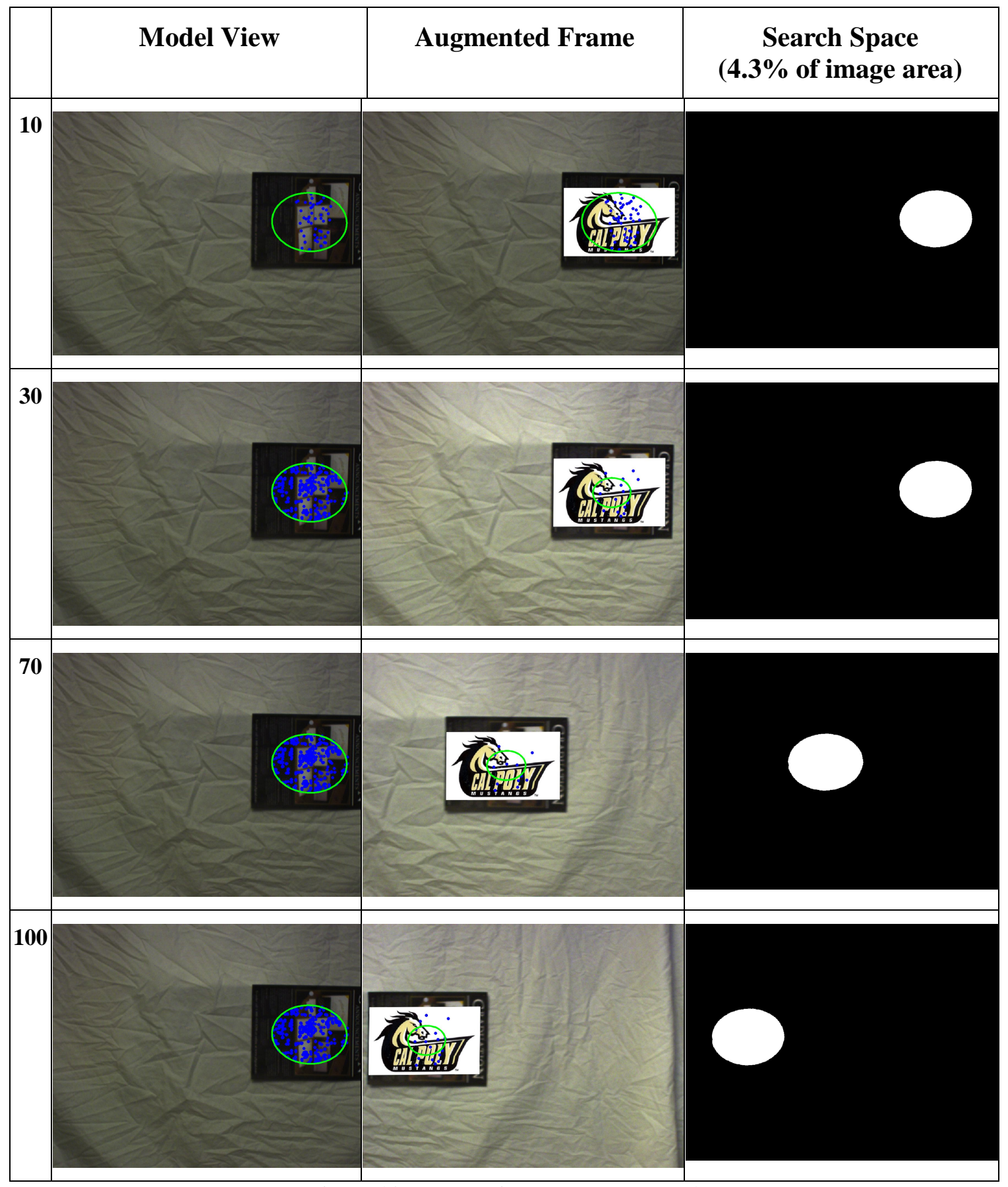

Figure 28: Translation Images, Indoor 1 


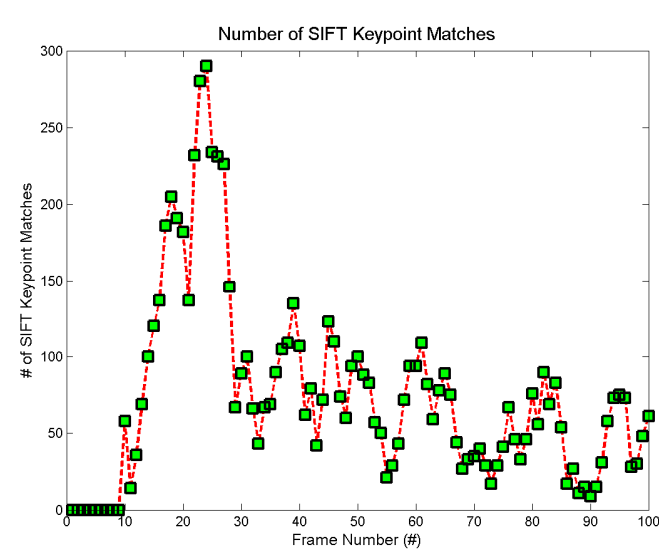

a)

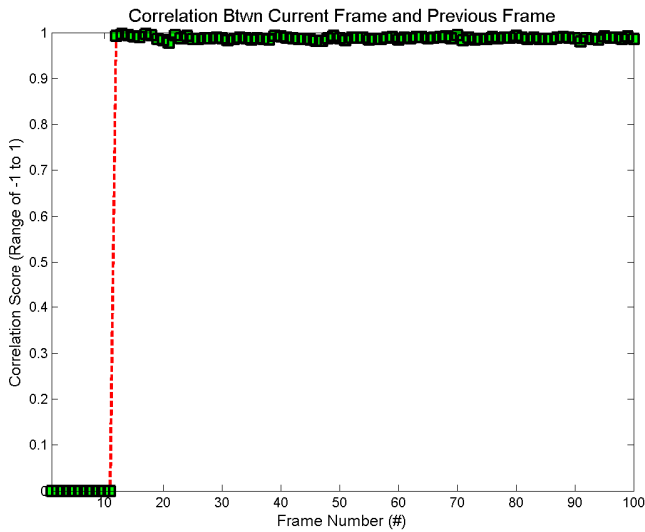

c)

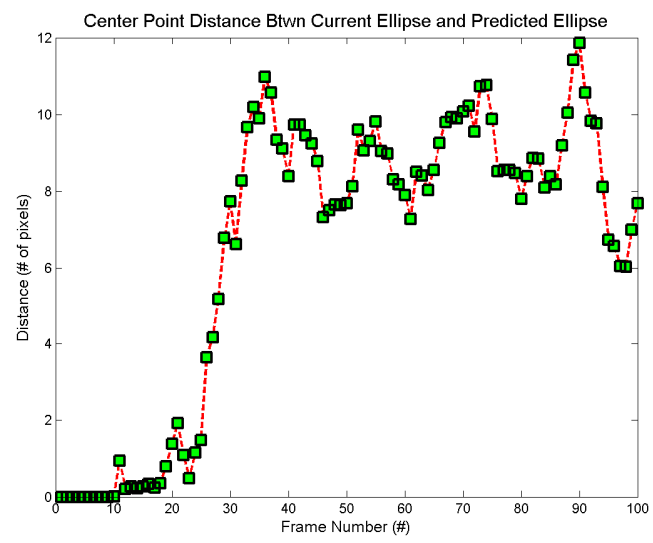

b)

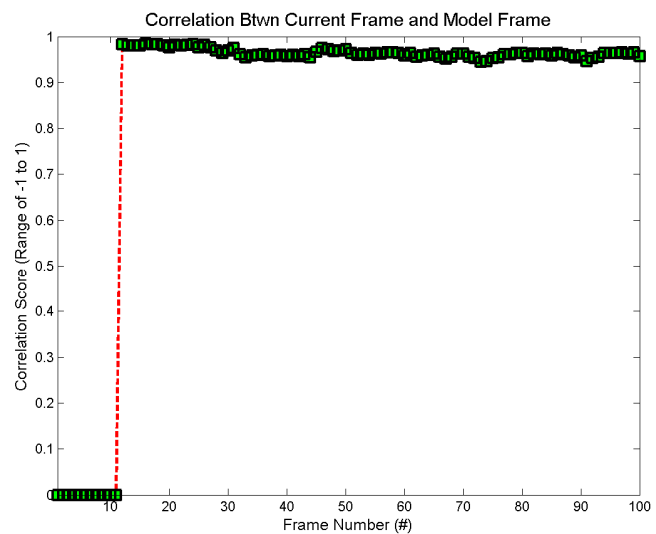

d)

Figure 29: Translation Plots Indoor 1, a) SIFT Keypoint Matches, b) Center Point Pixel Distances, c) Cross Correlation Btwn Current and Previous Frame, d) Cross Correlation Btwn Current and Model Frame

Table 9: Translation Data Indoor 1

\begin{tabular}{|r|r|r|r|}
\hline $\begin{array}{c}\text { Mean of SIFT } \\
\text { Keypoint Matches }\end{array}$ & $\begin{array}{c}\text { Mean of Center } \\
\text { Point Pixel Distances }\end{array}$ & $\begin{array}{c}\text { Mean of Cross } \\
\text { Correlation Btwn } \\
\text { Current and Previous } \\
\text { Frame }\end{array}$ & $\begin{array}{c}\text { Mean of Cross } \\
\text { Correlation Btwn } \\
\text { Current and Model } \\
\text { Frame }\end{array}$ \\
\hline 75.46 & 6.591857 & 0.879035 & 0.858097 \\
\hline
\end{tabular}




\section{Translational Tests for All Scenes}

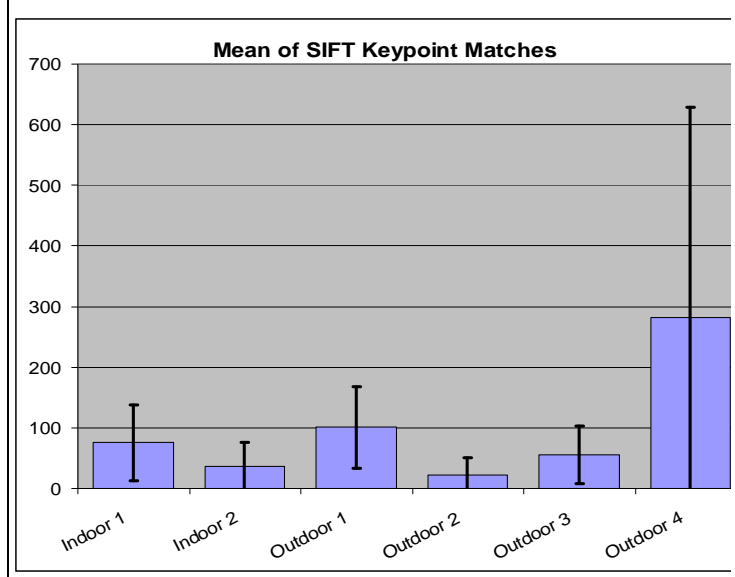

a)

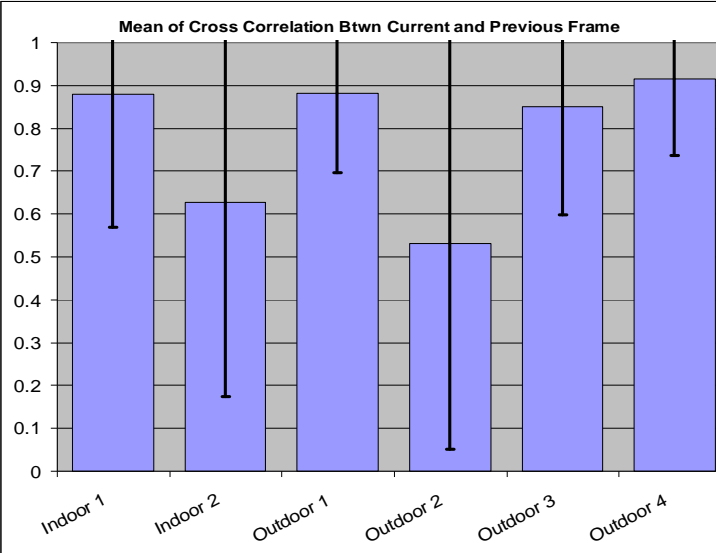

c)

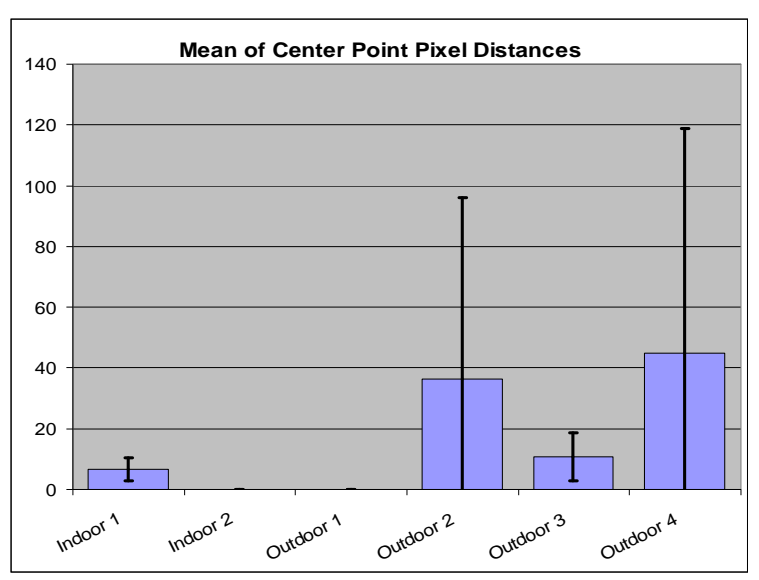

b)

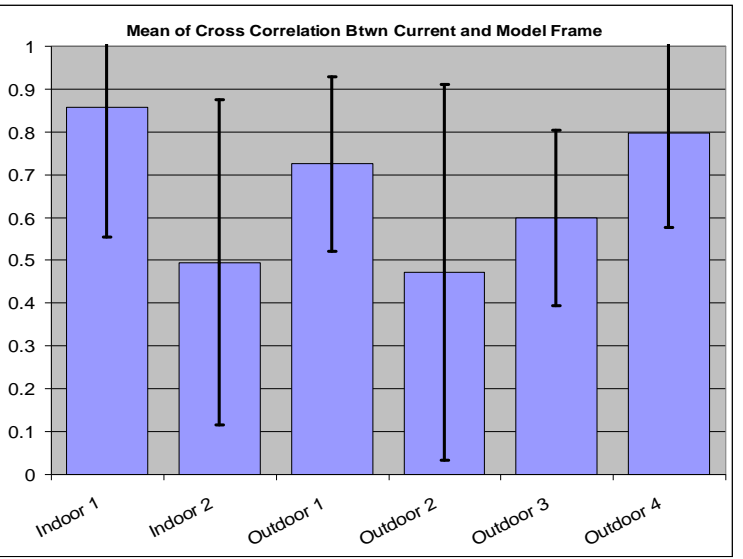

d)

Figure 30: Rotation Mean Plots, All Scenes, a) Mean of SIFT Keypoint Matches, b) Mean of Center Point Pixel Distances, c) Mean of Cross Correlation Btwn Current and Previous Frame, d) Mean of Cross Correlation Btwn Current and Model Frame 
Table 10: Translation Mean Data, All Scenes

\begin{tabular}{|c|c|c|c|c|c|}
\hline Scene & $\begin{array}{c}\text { Distance to } \\
\text { Object (ft) }\end{array}$ & $\begin{array}{c}\text { Mean of } \\
\text { SIFT } \\
\text { Keypoint } \\
\text { Matches }\end{array}$ & $\begin{array}{c}\text { Mean of } \\
\text { Center Point } \\
\text { Pixel } \\
\text { Distances }\end{array}$ & $\begin{array}{c}\text { Mean of Cross } \\
\text { Correlation } \\
\text { Btwn Current } \\
\text { and Previous } \\
\text { Frame }\end{array}$ & $\begin{array}{c}\text { Mean of Cross } \\
\text { Correlation } \\
\text { Btwn Current } \\
\text { and Model } \\
\text { Frame }\end{array}$ \\
\hline Ideal & - & 1185.85 & 0.006463 & 0.98 & 0.98 \\
\hline Indoor 1 & 5 & 75.46 & 6.591857 & 0.879035 & 0.858097 \\
\hline Indoor 2 & 6 & 36.04839 & 0 & 0.627798 & 0.495391 \\
\hline Outdoor 1 & 15 & 100.8696 & 0 & 0.881494 & 0.725747 \\
\hline Outdoor 2 & 12 & 21.51765 & 36.50076 & 0.531731 & 0.472762 \\
\hline Outdoor 3 & 12 & 55.5625 & 10.7158 & 0.851296 & 0.599248 \\
\hline Outdoor 4 & 12 & 281.4674 & 44.82857 & 0.915956 & 0.797089 \\
\hline & $\begin{array}{l}\text { Mean } \\
\text { Totals: }\end{array}$ & 95.15425 & 16.4395 & 0.781218 & 0.658056 \\
\hline
\end{tabular}

Note: Means do NOT include Ideal scene

\section{Translation Test Analysis}

The mean cross correlation score between the current frame and model frame for translation is 0.658 compared to a score of 0.608 for all tests. In three out of six real world tests the score between the current frame and model frame is greater than 0.7 for translation. This is the case for the scenes with the low blur, and consistent illumination: Indoor 1, Outdoor 1, and Outdoor 2. The cross-correlation score compared to the previous frame is about the same as across all the tests, at 0.78 . This indicates that the augmentation is consistent from frame to frame, but may be slowly drifting from the original model frame. This is not necessarily bad, because the original model frame has a low number of SIFT keypoints compared to the full set developed through the method.

The example case above (Indoor 1, Figure 29) has the highest cross correlation score compared to the model frame at 0.858 . It has low blur and good focus on the object being augmented, but the illumination does change noticeably from frame to frame as the camera adjusts to the low light conditions. This is a good indicator that changes in illumination are manageable in a low texture environment with low blur. 
Indoor 2 and Outdoor 2 have the lowest cross correlation scores compared to the model frame at 0.495 and 0.473 respectively. Indoor 2 has low light conditions and noticeable blur, while Outdoor 2 has inconsistent and saturated lighting with severe blurring. However, these correlations are still near 50\%, indicating that the augmentation is stable with a low chance of failure.

The number of SIFT keypoint matches varies greatly from scene to scene with a low mean of about 21 and a high mean of about 281 . This is affected by the size of the augmented region, as the texture of the surface being augmented. Low texture scenes such as Indoor 2 produce low mean SIFT keypoint matches, in this case around 36 across all augmented frames. Scenes with low keypoint matches also tend to have lower cross correlation scores, which is to be expected.

Overall the method performs well with changes in translation. 
Rotation Testing and Results

Representative Rotation Test: Indoor 1, D = 5ft

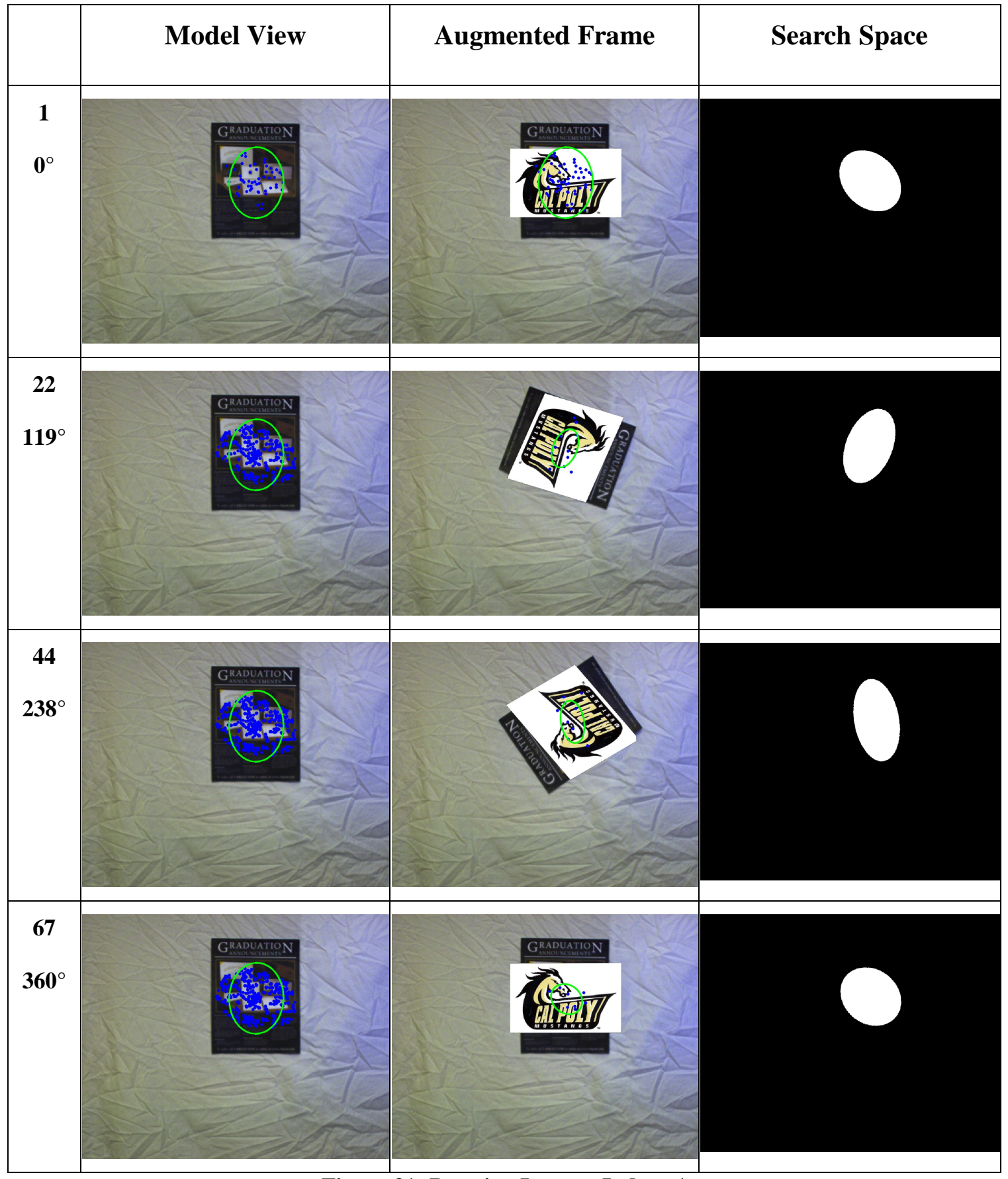

Figure 31: Rotation Images, Indoor 1 
Start Frame (1) $=0^{\circ}$, End Frame (67) $=360^{\circ}$, Delta $=5.4^{\circ}$ per Frame

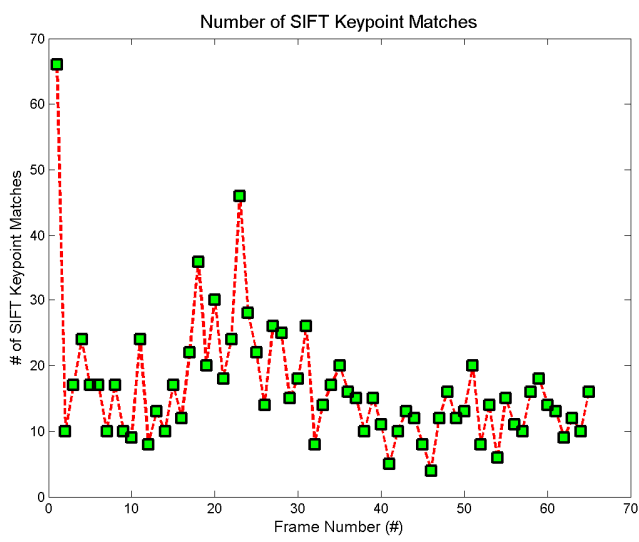

a)

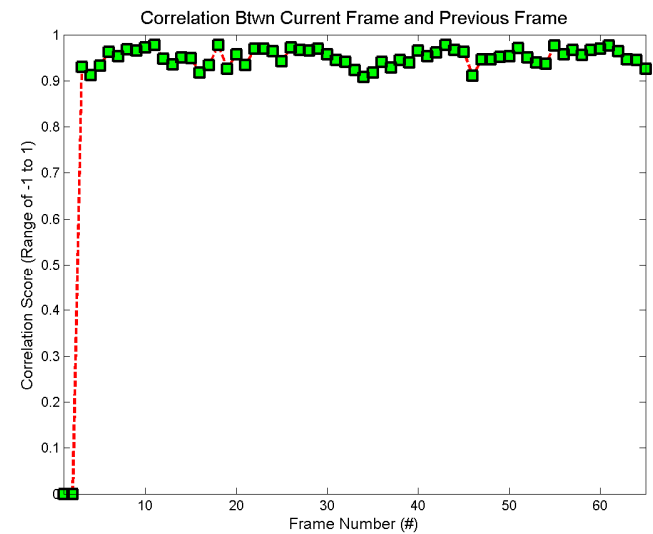

c)

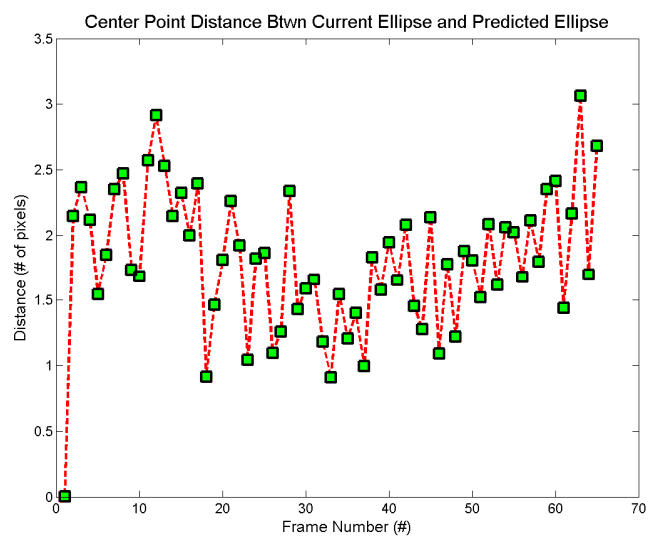

b)

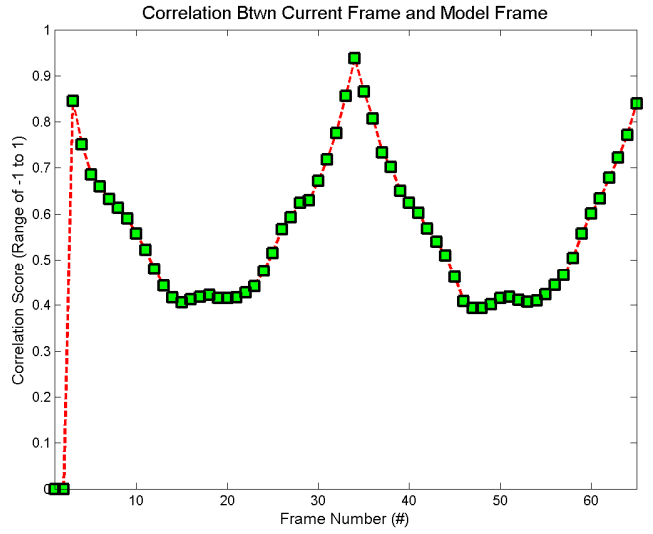

d)

Figure 32: Rotation Plots Outdoor 1, a) SIFT Keypoint Matches, b) Center Point Pixel Distances, c) Cross Correlation Btwn Current and Previous Frame, d) Cross Correlation Btwn Current and Model Frame

Table 11: Rotation Data Indoor 1

\begin{tabular}{|r|r|r|r|}
\hline $\begin{array}{c}\text { Mean of SIFT } \\
\text { Keypoint Matches }\end{array}$ & $\begin{array}{c}\text { Mean of Center } \\
\text { Point Pixel Distances }\end{array}$ & $\begin{array}{c}\text { Mean of Cross } \\
\text { Correlation Btwn } \\
\text { Current and Previous } \\
\text { Frame }\end{array}$ & $\begin{array}{c}\text { Mean of Cross } \\
\text { Correlation Btwn } \\
\text { Current and Model } \\
\text { Frame }\end{array}$ \\
\hline 16.52308 & 1.804508 & 0.921468 & 0.54925 \\
\hline
\end{tabular}




\section{Rotation Tests for All Scenes}

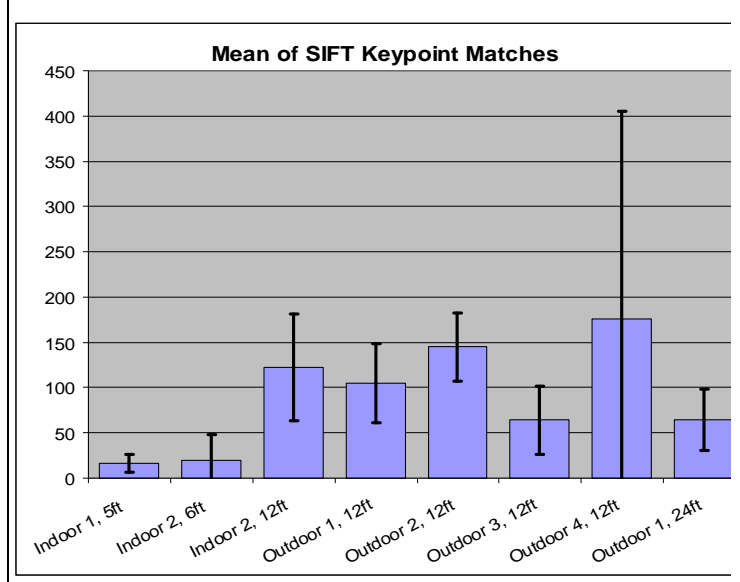

a)

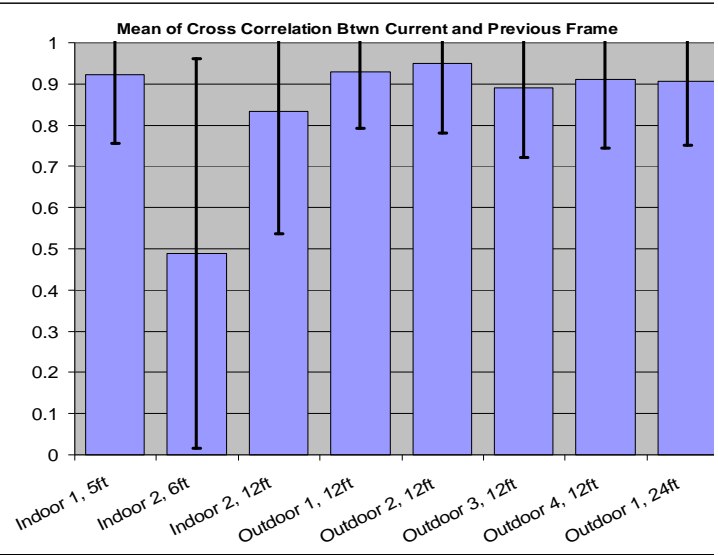

c)

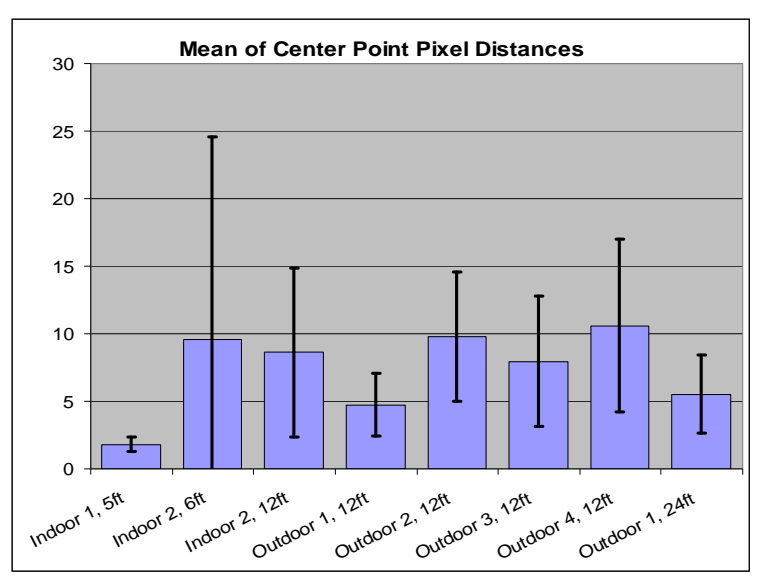

b)

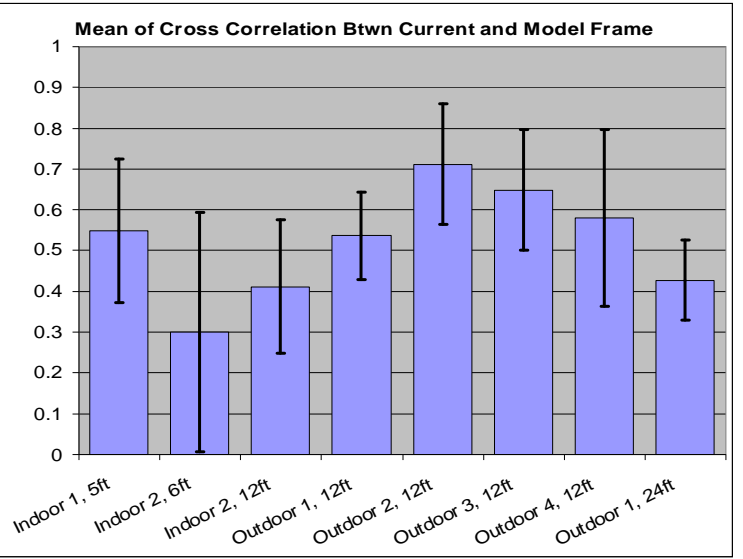

d)

Figure 33: Rotation Mean Plots, All Scenes, a) Mean of SIFT Keypoint Matches, b) Mean of Center Point Pixel Distances, c) Mean of Cross Correlation Btwn Current and Previous Frame, d) Mean of Cross Correlation Btwn Current and Model Frame 
Table 12: Rotation Mean Data, All Scenes

\begin{tabular}{|l|c|r|r|r|r|}
\hline Scene & $\begin{array}{c}\text { Distance to } \\
\text { Object (ft) }\end{array}$ & $\begin{array}{c}\text { Mean of } \\
\text { SIFT } \\
\text { Keypoint } \\
\text { Matches }\end{array}$ & $\begin{array}{c}\text { Mean of } \\
\text { Center Point } \\
\text { Pixel } \\
\text { Distances }\end{array}$ & $\begin{array}{c}\text { Mean of Cross } \\
\text { Correlation } \\
\text { Btwn Current } \\
\text { and Previous } \\
\text { Frame }\end{array}$ & $\begin{array}{c}\text { Mean of Cross } \\
\text { Correlation } \\
\text { Btwn Current } \\
\text { and Model } \\
\text { Frame }\end{array}$ \\
\hline Ideal & - & 333.6 & 0.130074 & 0.933787 & 0.837808 \\
\hline Indoor 1 & 5 & 16.52308 & 1.804508 & 0.921468 & 0.54925 \\
\hline Indoor 2 & 6 & 19.69 & 9.599946 & 0.488831 & 0.299184 \\
\hline Indoor 2 & 12 & 122.29 & 8.620545 & 0.834187 & 0.41181 \\
\hline Outdoor 1 & 12 & 104.49 & 4.739818 & 0.92876 & 0.536275 \\
\hline Outdoor 1 & 24 & 64.7 & 5.529365 & 0.905468 & 0.427466 \\
\hline Outdoor 2 & 12 & 144.93 & 9.778651 & 0.949811 & 0.711703 \\
\hline Outdoor 3 & 12 & 64.08696 & 7.962489 & 0.890499 & 0.648316 \\
\hline Outdoor 4 & 12 & 176.07 & 10.59885 & 0.911436 & 0.580254 \\
\hline & Mean & 89.0975 & 7.329272 & 0.853808 & 0.520532 \\
\hline
\end{tabular}

Note: Means do NOT include Ideal scene

\section{Rotation Test Analysis}

The mean of the SIFT keypoint matches for rotation is slightly higher than the overall tests at 89 matches. SIFT keypoint matches are above 60 for all real world tests, except for Indoor 1 and Indoor 2. In the case of Indoor 1, this is likely due to the small area, and therefore small number of model view SIFT keypoints. In the case of Indoor 2, Figure 59 shows that the model view frame has very low light conditions compared to the majority of subsequent frames. Despite the large number of SIFT keypoint matches in the model view frame, this severe change in illumination causes very few keypoint matches in later frames.

The mean center point pixel distance between the model frame ellipse and current ellipse is 7.33 compared to a mean of 15.92 for all tests. This indicates that in purely rotational testing the center point stays relatively stable, which is to be expected by the nature of the test. 
The method handles change is rotation well, with one visible exception. Skewing of the image being augmented into the scene is clearly visible. This issue is quantified by the difference in the cross-correlation score relative to the previous frame vs. the crosscorrelation score relative to the current frame. Compared to the mean across all the tests, rotation has a high cross-correlation score relative to the previous frame at 0.854 compared to 0.779 overall. However, it has a low cross-correlation score relative to the model frame at 0.52 compared to 0.608 overall. This corresponds to a minor skewing in the graphic being augmented into the scene. In the example case, Indoor 1, the skewing gets worse as the orientation approaches the $80^{\circ}$ to $130^{\circ}$ range, improves from $130^{\circ}$ to $180^{\circ}$, and repeats a symmetrical trend toward $360^{\circ}$, as indicated by Figure $62 \mathrm{~d}$. This is most likely due to localization and matching issues with the SIFT method. Closely localized, but unique SIFT keypoints, may have a false match with one another. If this occurs between two points simultaneously from one frame to the next, a full $180^{\circ}$ rotation may occur based off of just these two points. This issue is minimized due to the large number of correct SIFT keypoint matches, but is most noticeable in the case of rotation because of the potential for extreme angle changes based off of just two false matches. Although this does degrade the performance and accuracy of the affine transform under rotational changes, the overall skewing is somewhat negligible to the human eye. 
Scale Testing and Results

Representative Scale Test: Indoor 1

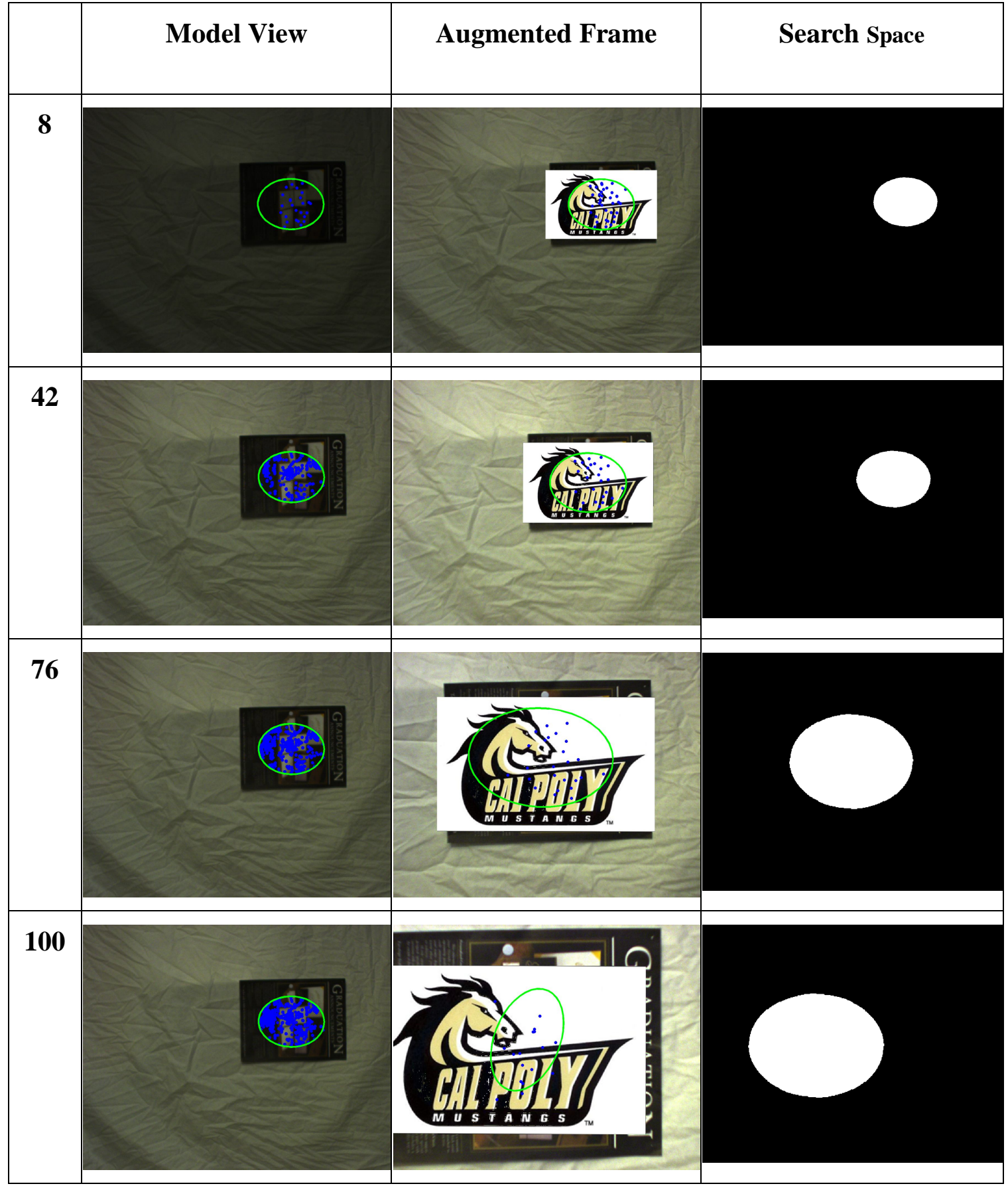

Figure 34: Scale Images, Indoor 1 


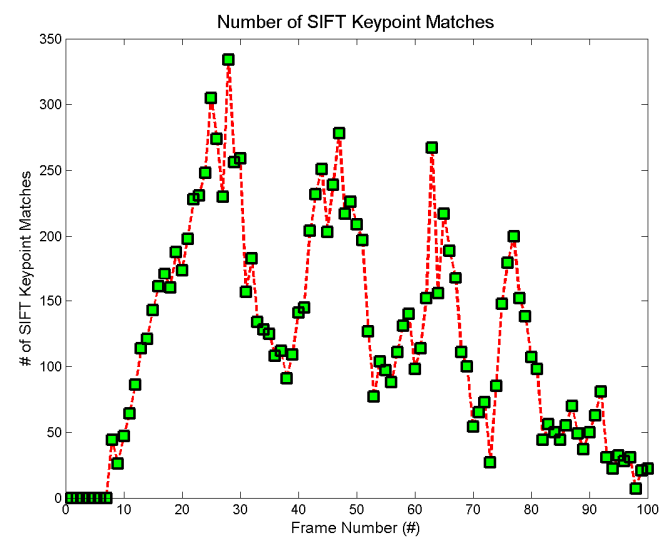

a)

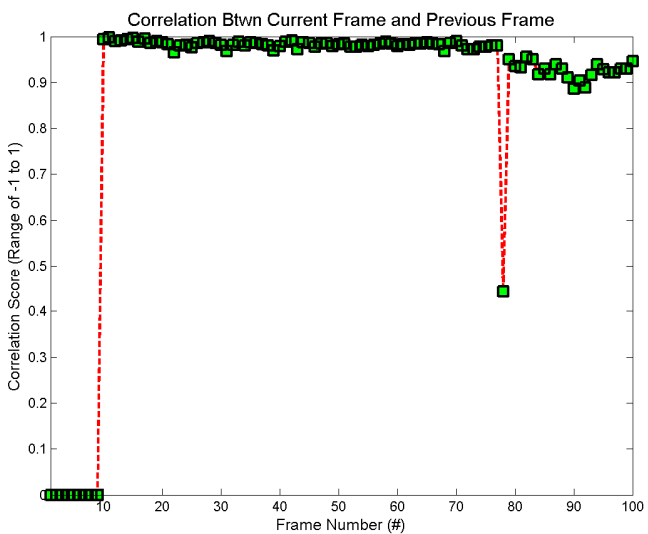

c)

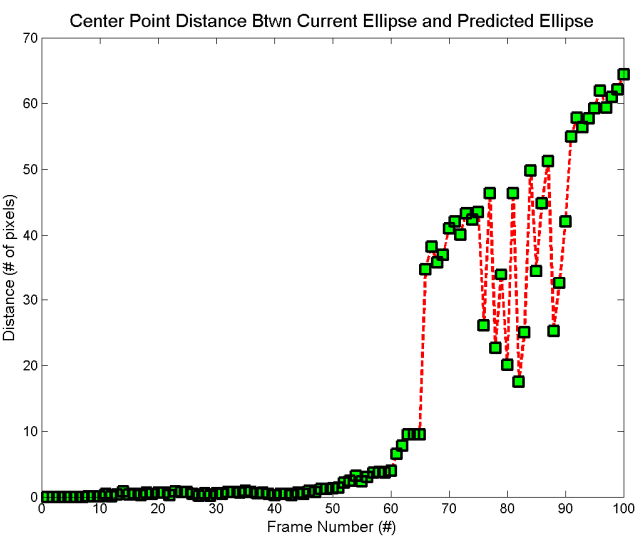

b)

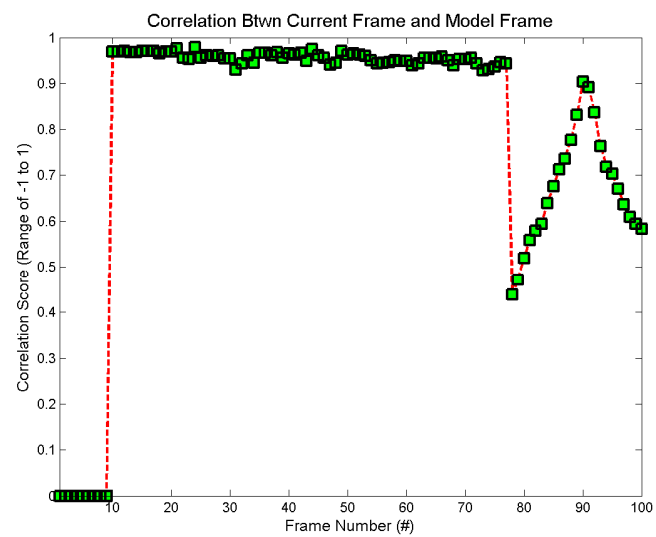

d)

Figure 35: Scale Plots Indoor 1, a) SIFT Keypoint Matches, b) Center Point Pixel Distances, c) Cross Correlation Btwn Current and Previous Frame, d) Cross Correlation Btwn Current and Model Frame

Table 13: Scale Data Indoor 1

\begin{tabular}{|r|r|r|r|}
\hline $\begin{array}{c}\text { Mean of SIFT } \\
\text { Keypoint Matches }\end{array}$ & $\begin{array}{c}\text { Mean of Center } \\
\text { Point Pixel Distances }\end{array}$ & $\begin{array}{c}\text { Mean of Cross } \\
\text { Correlation Btwn } \\
\text { Current and Previous } \\
\text { Frame }\end{array}$ & $\begin{array}{c}\text { Mean of Cross } \\
\text { Correlation Btwn } \\
\text { Current and Model } \\
\text { Frame }\end{array}$ \\
\hline 123.18 & 16.06933 & 0.876745 & 0.804756 \\
\hline
\end{tabular}




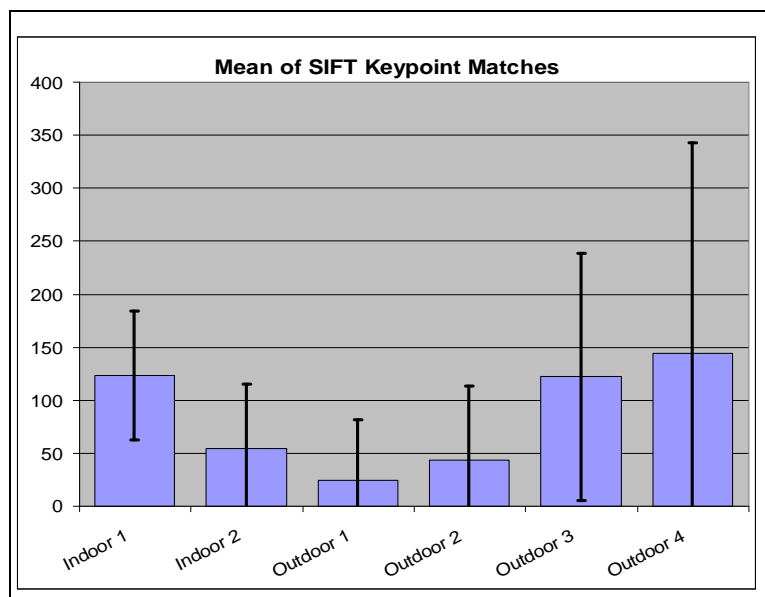

a)

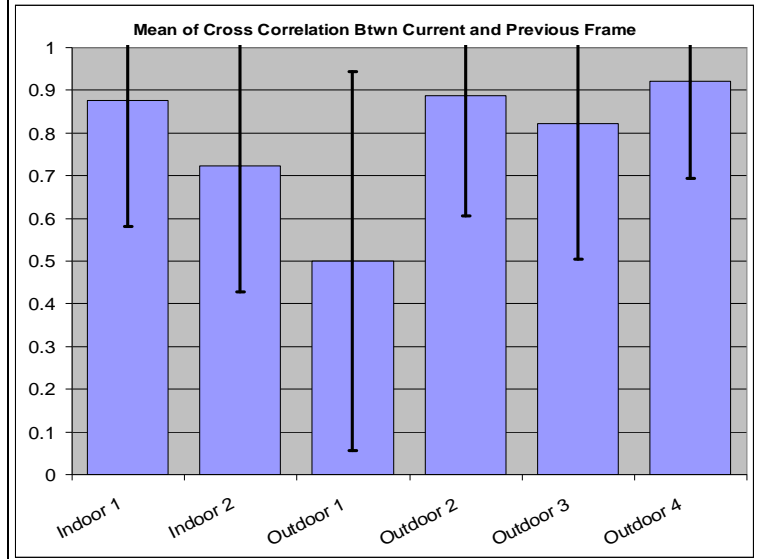

c)

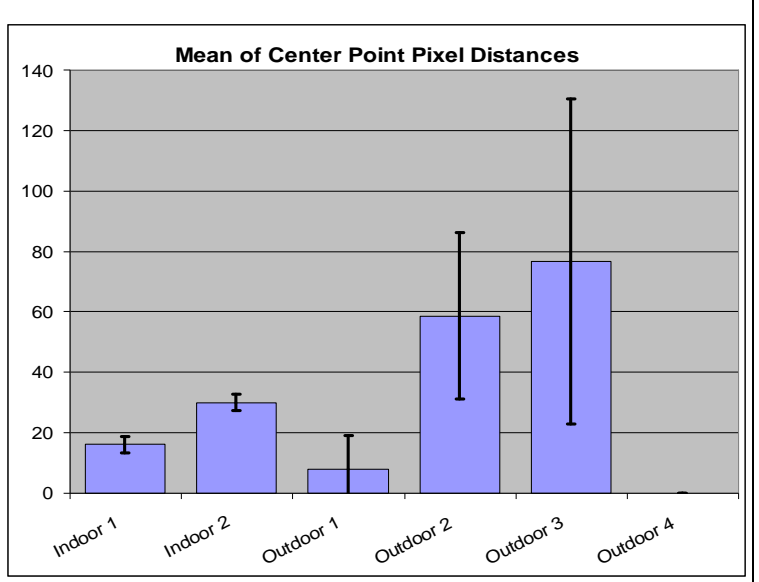

b)

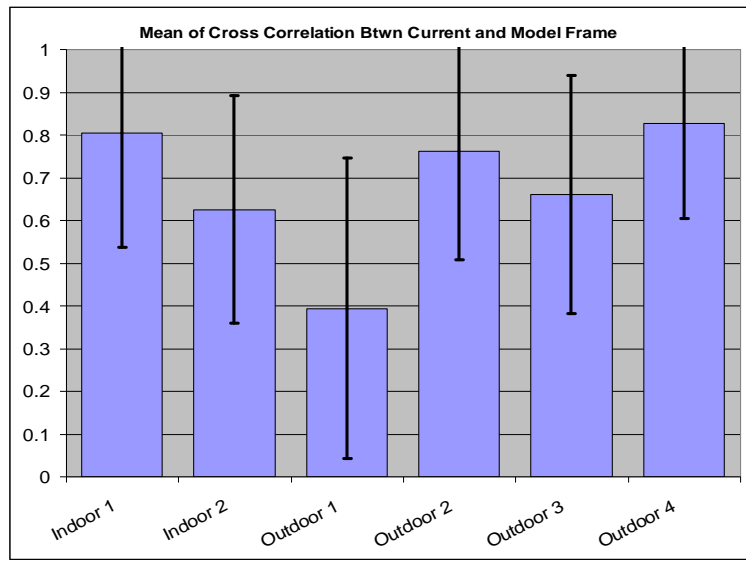

d)

Figure 36: Scale Mean Plots, All Scenes, a) Mean of SIFT Keypoint Matches, b) Mean of Center Point Pixel Distances, c) Mean of Cross Correlation Btwn Current and Previous Frame, d) Mean of Cross Correlation Btwn Current and Model Frame 
Table 14: Scale Mean Data, All Scenes

\begin{tabular}{|c|c|c|c|c|c|c|}
\hline Scene & $\begin{array}{c}\text { Starting } \\
\text { Distance } \\
\text { (ft) }\end{array}$ & $\begin{array}{c}\text { Ending } \\
\text { Distance } \\
\text { (ft) }\end{array}$ & $\begin{array}{l}\text { Mean of } \\
\text { SIFT } \\
\text { Keypoint } \\
\text { Matches }\end{array}$ & $\begin{array}{c}\text { Mean of } \\
\text { Center } \\
\text { Point Pixel } \\
\text { Distances }\end{array}$ & $\begin{array}{c}\text { Mean of } \\
\text { Cross } \\
\text { Correlation } \\
\text { Btwn } \\
\text { Current and } \\
\text { Previous } \\
\text { Frame } \\
\end{array}$ & $\begin{array}{c}\text { Mean of } \\
\text { Cross } \\
\text { Correlation } \\
\text { Btwn } \\
\text { Current and } \\
\text { Model } \\
\text { Frame } \\
\end{array}$ \\
\hline \multicolumn{7}{|l|}{ Ideal } \\
\hline Indoor 1 & 2 & 6 & 123.18 & 16.06933 & 0.876745 & 0.804756 \\
\hline Indoor 2 & 3 & 9 & 54.32941 & 30.04514 & 0.723223 & 0.625814 \\
\hline Outdoor 1 & 7 & 21 & 24.7619 & 7.995469 & 0.49941 & 0.39367 \\
\hline Outdoor 2 & 7 & 21 & 43.28 & 58.65419 & 0.887533 & 0.762544 \\
\hline Outdoor 3 & 3 & 24 & 122.3924 & 76.58836 & 0.822148 & 0.660416 \\
\hline Outdoor 4 & 3 & 24 & 143.8451 & 0 & 0.920767 & 0.82782 \\
\hline & & $\begin{array}{l}\text { Mean } \\
\text { Totals: }\end{array}$ & 66.69514 & 23.99144 & 0.701489 & 0.646696 \\
\hline
\end{tabular}

Note: Means do NOT include Ideal scene

\section{Scale Test Analysis}

The method performs better in scale testing than in any other tests when compared to the mean of all tests. This is evident in the above average cross-correlation score relative to the model frame of 0.647 compared to 0.608 overall. The crosscorrelation score relative to the previous frame is low at 0.701 compared to the overall score of 0.779 . This is likely due to the fact that scale change is the one case where the search space is varying in area significantly from frame to frame. This is important, because the search space is the area that is used to calculate the cross-correlation scores. Even if the area of the search space only increases by a few percent, the cross-correlation score is significantly affected. The fact that the cross-correlation score relative to the previous frame is the one that is lower than in the overall tests reveals that the issue is on a frame to frame basis.

The mean of SIFT keypoint matches at 66.7 is lower than the mean over all tests at 83.6. This is due to several factors. During scaling there is a loss in resolution of the 
image as the camera moves further from the region being augmented. Inevitably this loss in resolution will lead to some keypoints becoming virtually invisible, despite the scaleinvariant nature of the SIFT algorithm.

The center point pixel difference for the model ellipse compared to the current ellipse is higher at 24 pixels, compared to about 16 pixels for the overall mean pixel difference of all tests. This indicates that there is some drift in the SIFT algorithm as resolution decreases, but could also be due to the fact that the MSER algorithm becomes inaccurate with significant blurring. 


\section{Viewpoint Angle Testing and Results}

Viewpoint Angle Test 1

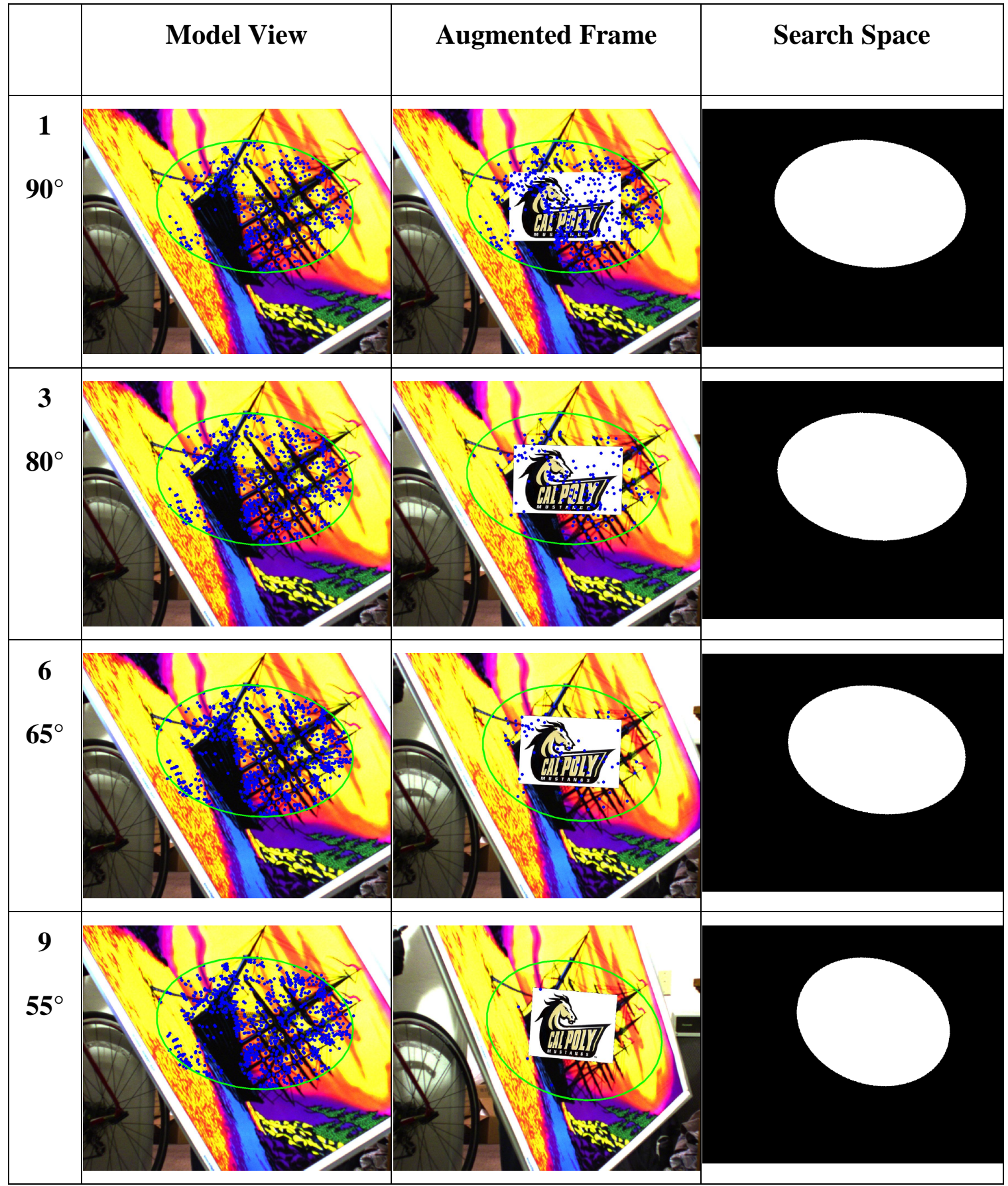

Figure 37: Images, Viewpoint Angle Test 1 
Start Frame $(1)=90^{\circ}$, End Frame $(9)=55^{\circ}$, Delta $=5^{\circ}$ per Frame

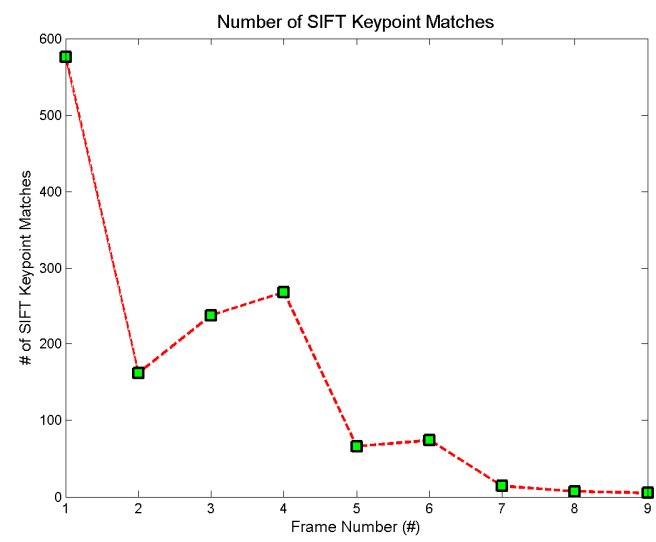

a)

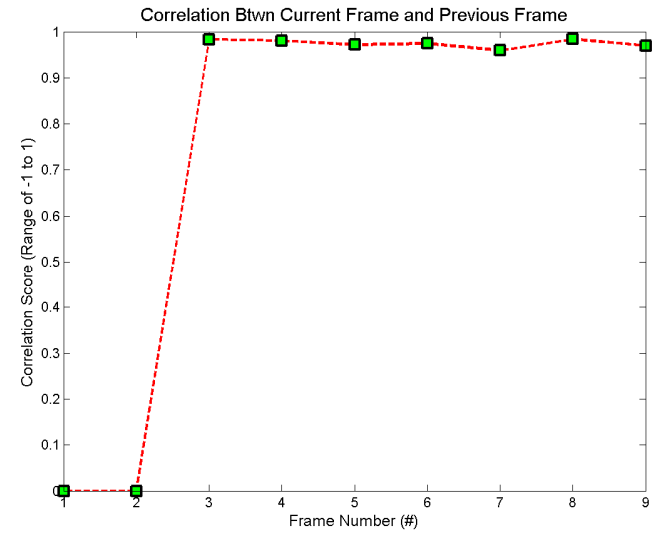

c)

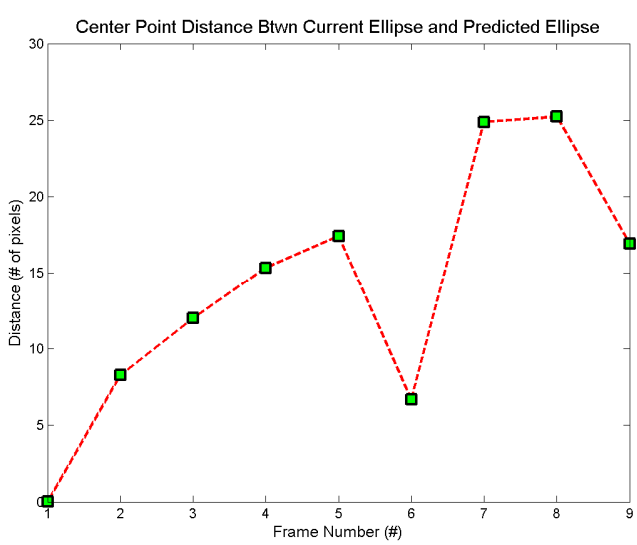

b)

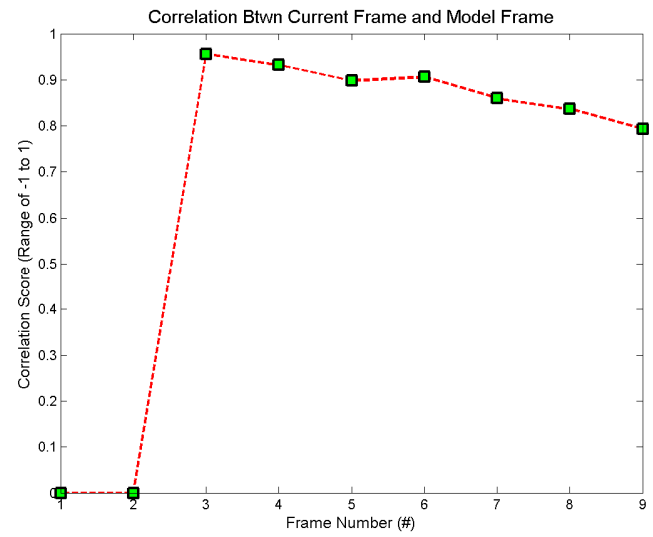

d)

Figure 38: Viewpoint Angle Test 1 Plots, a) SIFT Keypoint Matches, b) Center Point Pixel Distances, c) Cross Correlation Btwn Current and Previous Frame, d) Cross Correlation Btwn Current and Model Frame

Table 15: Viewpoint Angle Test 1 Data

\begin{tabular}{|r|r|r|r|}
\hline $\begin{array}{c}\text { Mean of SIFT } \\
\text { Keypoint Matches }\end{array}$ & $\begin{array}{c}\text { Mean of Center } \\
\text { Point Pixel Distances }\end{array}$ & $\begin{array}{c}\text { Mean of Cross } \\
\text { Correlation Btwn } \\
\text { Current and Previous } \\
\text { Frame }\end{array}$ & $\begin{array}{c}\text { Mean of Cross } \\
\text { Correlation Btwn } \\
\text { Current and Model } \\
\text { Frame }\end{array}$ \\
\hline 156.4444 & 14.08376 & 0.757995 & 0.68688 \\
\hline
\end{tabular}


Viewpoint Angle Test 2

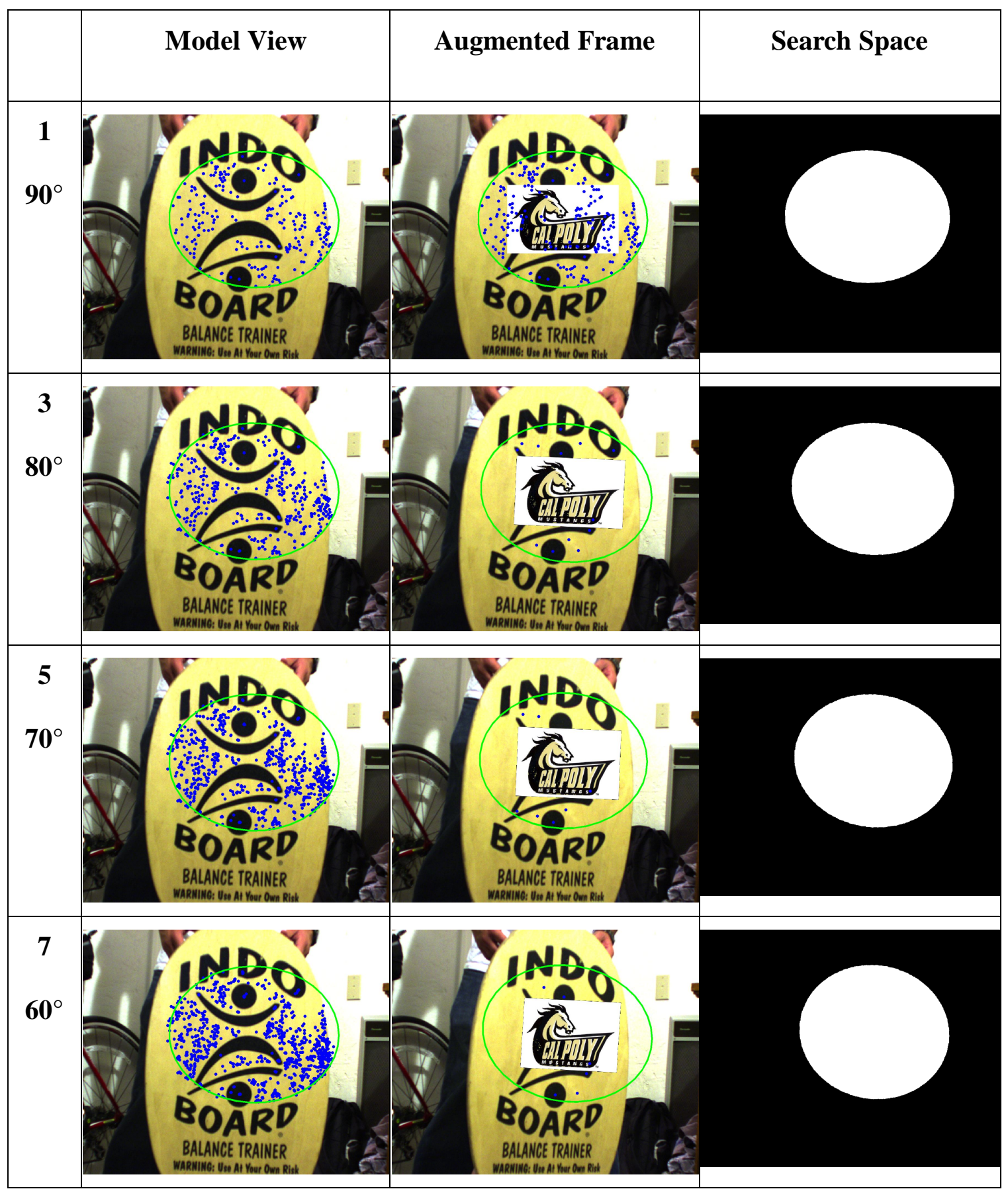

Figure 39: Images, Viewpoint Angle Test 2 
Start Frame $(1)=90^{\circ}$, End Frame $(7)=60^{\circ}$, Delta $=5^{\circ}$ per Frame

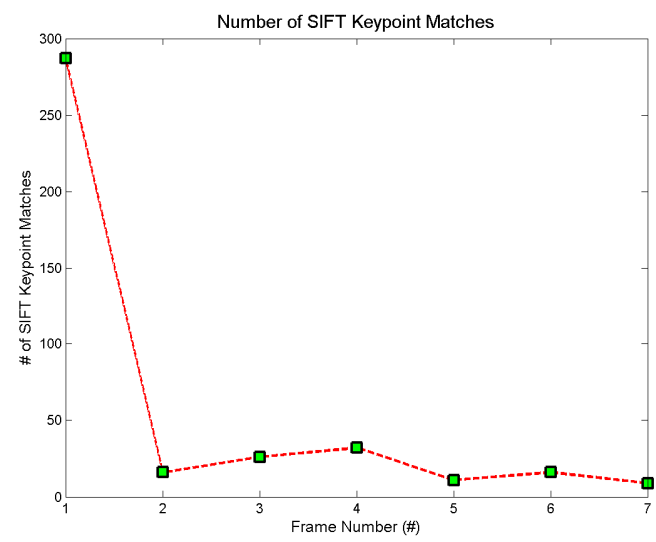

a)

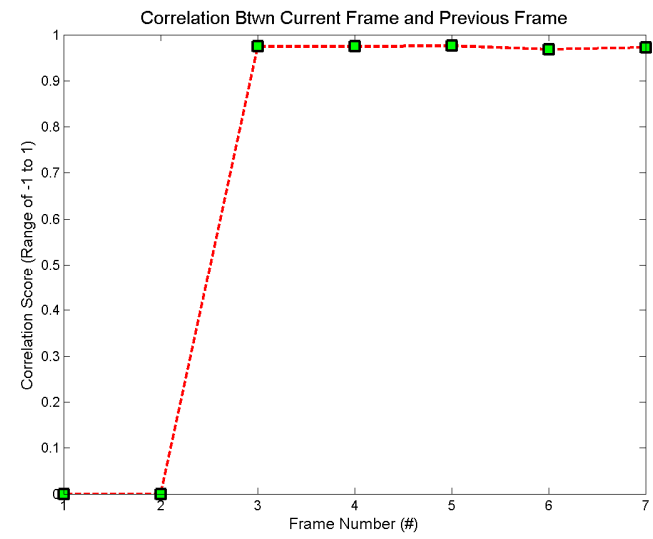

c)

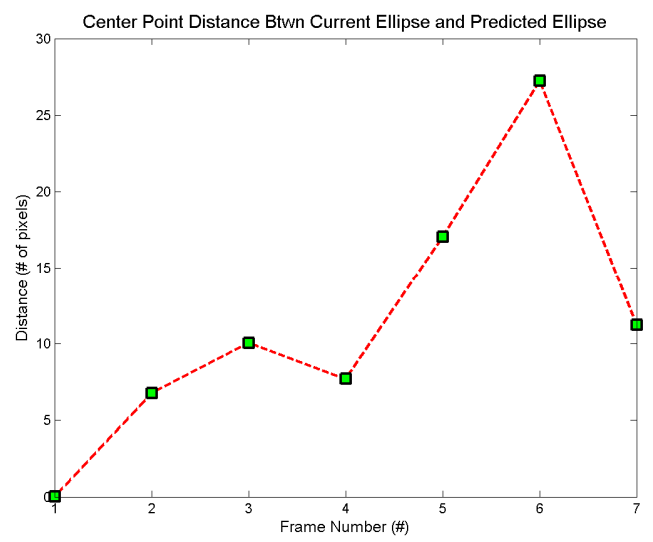

b)

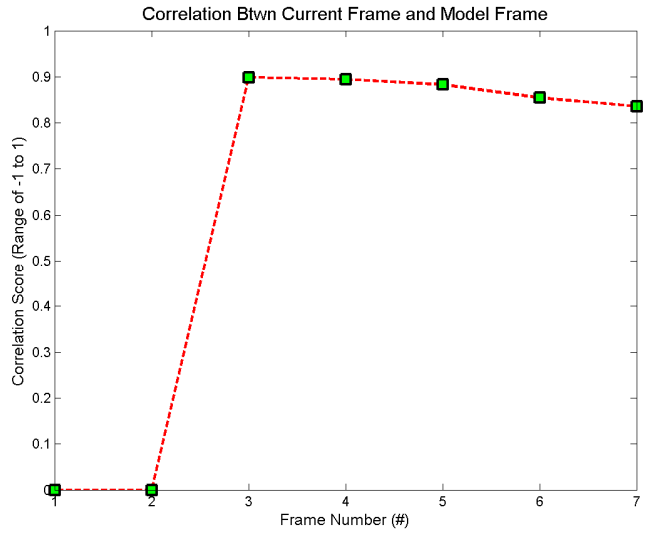

d)

Figure 40: Viewpoint Angle Test 2 Plots, a) SIFT Keypoint Matches, b) Center Point Pixel Distances, c) Cross Correlation Btwn Current and Previous Frame, d) Cross Correlation Btwn Current and Model Frame

Table 16: Viewpoint Angle Test 2 Data

\begin{tabular}{|r|r|r|r|}
\hline $\begin{array}{c}\text { Mean of SIFT } \\
\text { Keypoint Matches }\end{array}$ & $\begin{array}{c}\text { Mean of Center } \\
\text { Point Pixel Distances }\end{array}$ & $\begin{array}{c}\text { Mean of Cross } \\
\text { Correlation Btwn } \\
\text { Current and Previous } \\
\text { Frame }\end{array}$ & $\begin{array}{c}\text { Mean of Cross } \\
\text { Correlation Btwn } \\
\text { Current and Model } \\
\text { Frame }\end{array}$ \\
\hline 56.71429 & 11.43297 & 0.694952 & 0.623804 \\
\hline
\end{tabular}




\section{Viewpoint Angle Test Analysis}

The viewpoint angle tests provide an understanding of how the method handles changes in viewpoint of the camera relative to the region being augmented. Data for both tests shown in Figure 38a and Figure 40a indicate that viewpoint angle change results in significantly fewer SIFT keypoint matches each frame, even after only $5^{\circ}$ of rotation. Subplot d) for both Figures show that cross-correlation between current and model view frames drops of gradually, with no clear indication that the region is about to be lost. Although this makes viewpoint angle changes an issue for the method, as soon as the camera returns to a viewpoint angle within the methods ability, the current frame will again match the model frame and the augmentation will be immediately restored. The range of viewpoint angle change is from $90^{\circ}$ (camera looking straight on toward region being augmented) to $35^{\circ}$ or a delta of $45^{\circ}$. This essentially equates to a full $90^{\circ}$ of possible angles the method can operate under (taking symmetry into account).

The mean of both cross-correlation measures is very high at above 0.8 excluding the first point which should be neglected (Figure $40 \mathrm{c}$ and d). This indicates that the system is very robust at detecting matches with the model frame as long as the viewpoint angle is with the acceptable range of $45^{\circ}$, holding all other variables constant. 
Occlusion Testing and Results

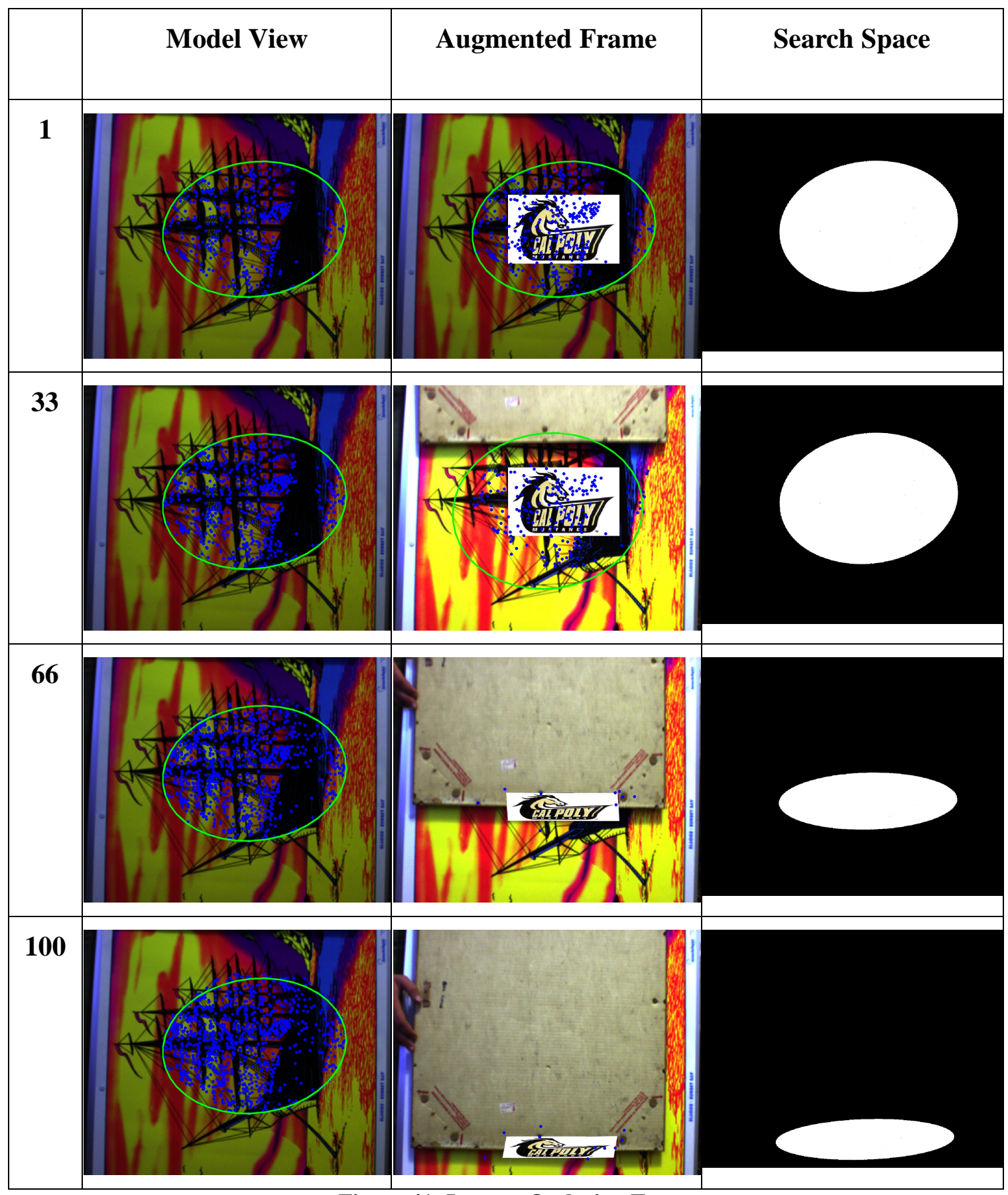

Figure 41: Images, Occlusion Test 


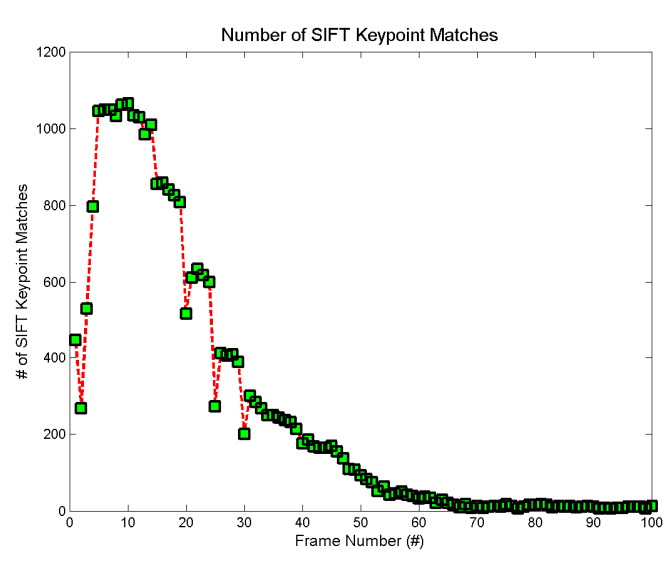

a)

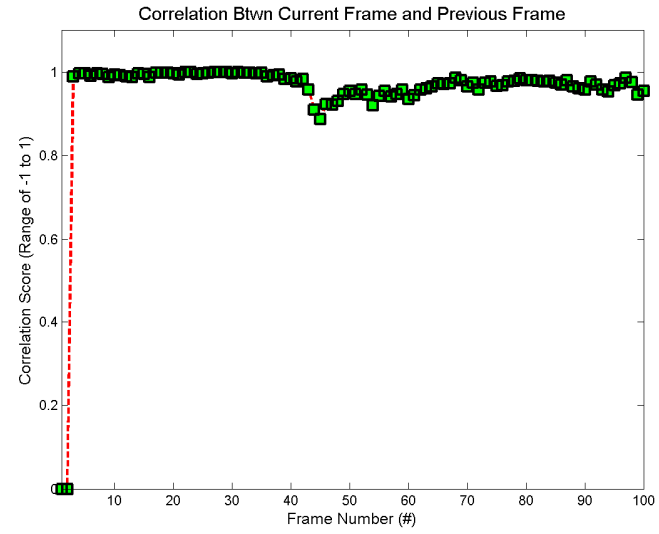

c)

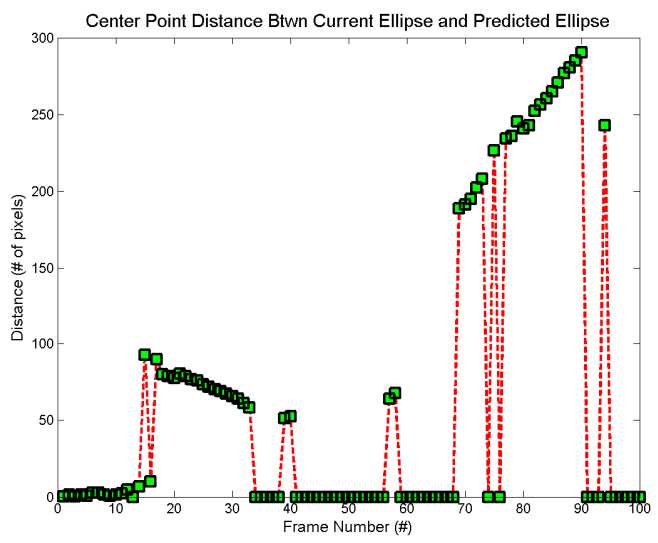

b)

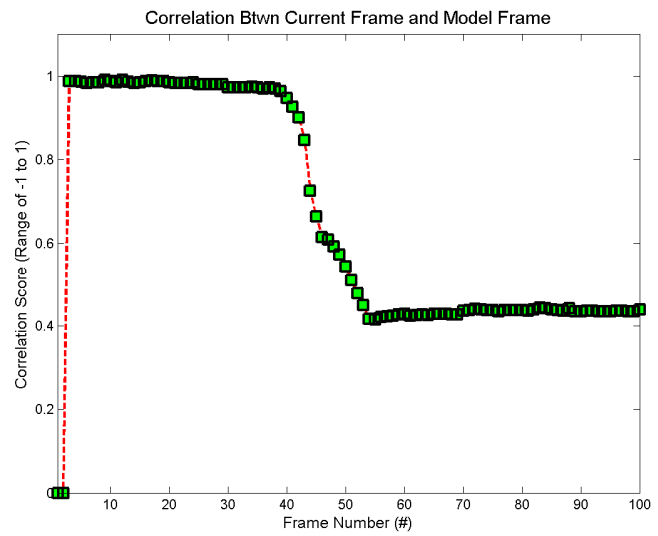

d)

Figure 42: Occlusion Test Plots, a) SIFT Keypoint Matches, b) Center Point Pixel Distances, c) Cross Correlation Btwn Current and Previous Frame, d) Cross Correlation Btwn Current and Model Frame

Table 17: Occlusion Test Data

\begin{tabular}{|r|r|r|r|}
\hline $\begin{array}{c}\text { Mean of SIFT } \\
\text { Keypoint Matches }\end{array}$ & $\begin{array}{c}\text { Mean of Center } \\
\text { Point Pixel Distances }\end{array}$ & $\begin{array}{c}\text { Mean of Cross } \\
\text { Correlation Btwn } \\
\text { Current and Previous } \\
\text { Frame }\end{array}$ & $\begin{array}{c}\text { Mean of Cross } \\
\text { Correlation Btwn } \\
\text { Current and Model } \\
\text { Frame }\end{array}$ \\
\hline 265.93 & 66.94664 & 0.954884 & 0.660545 \\
\hline
\end{tabular}




\section{Occlusion Test Analysis}

Individually the SIFT method and MSER detector find many regions and therefore are potentially very good at handling occlusion. However, in the case of this method there is a scenario that can arise that makes the system sensitive to occlusion. An example of this is outlined in Figure 41. As can be seen in Figure 41 frame 33, the occluding surface can enter the search region defined by the MSER ellipse. If SIFT keypoints are found on this occluding surface (as they are in this example) there is a possibility that they will be added to the model frame. If this occurs and the occluding surface than moves, the affine transform is changed accordingly, and the augmentation is negatively affected. The method could easily be made more robust to this issue if SIFT keypoints where more strictly matched, thus not allowing keypoints from an occluding surface to be added to the model view. 


\section{Conclusions}

The method presented successfully augments regions under many different variable scenarios including varying surface textures, environmental texture, illumination, translation, rotation, and scale. It is most sensitive to changes in illumination and blur, which often cause SIFT keypoints from one frame to the next to vary to greatly from one another for successful matching.

The method is able to augment many different surface types, requiring only about 30 model view SIFT keypoints in order to perform stable augmentation across many frames. Changes in scale are handled the best as quantified by the highest cross-correlation scores. In fact, due to large changes in search area from frame to frame in changes of scale, the cross-correlation measure actually underestimates the performance of the method in this case. Changes in translation are handled almost equally well, but are somewhat hampered by viewpoint angle changes. The assumption that perspective affects can be ignored is less applicable under changes in viewpoint angle, which is the main cause of lower performance in augmentation for translation. Changes in rotation are the weakest point of the method, with noticeable skew occurring due to false SIFT keypoint matches. Occlusion can be an issue if an occluding surface gradually enters the search space of the region being augmented. However, this issue can easily be resolved by matching SIFT keypoints more strictly.

Finally, a comparison of the three main tests across all scenes vs. the same tests in the ideal scene in Table 18 show that the method is very strong under real world conditions. 
Table 18: Mean Data, All Tests, All Scenes

\begin{tabular}{|c|c|c|c|c|}
\hline Test & $\begin{array}{c}\text { Mean of SIFT } \\
\text { Keypoint } \\
\text { Matches }\end{array}$ & $\begin{array}{c}\text { Mean of Center } \\
\text { Point Pixel } \\
\text { Distances }\end{array}$ & $\begin{array}{l}\text { Mean of Cross } \\
\text { Correlation } \\
\text { Btwn Current } \\
\text { and Previous } \\
\text { Frame }\end{array}$ & $\begin{array}{l}\text { Mean of Cross } \\
\text { Correlation } \\
\text { Btwn Current } \\
\text { and Model } \\
\text { Frame }\end{array}$ \\
\hline All tests, all scenes & 83.64896 & 15.92007 & 0.778838 & 0.608428 \\
\hline Translation, all scenes & 95.15425 & 16.4395 & 0.781218 & 0.658056 \\
\hline Rotation, all scenes & 89.0975 & 7.329272 & 0.853808 & 0.520532 \\
\hline Scale, all scenes & 66.69514 & 23.99144 & 0.701489 & 0.646696 \\
\hline Translation, Ideal & 1185.85 & 0.006463 & 0.98 & 0.98 \\
\hline Rotation, Ideal & 333.6 & 0.130074 & 0.933787 & 0.837808 \\
\hline Scale, Ideal & 24.7619 & 7.995469 & 0.49941 & 0.39367 \\
\hline
\end{tabular}




\section{Appendix: Images and Data Plots for Additional Tests}

\section{Translation Tests}

Translation Test: Ideal

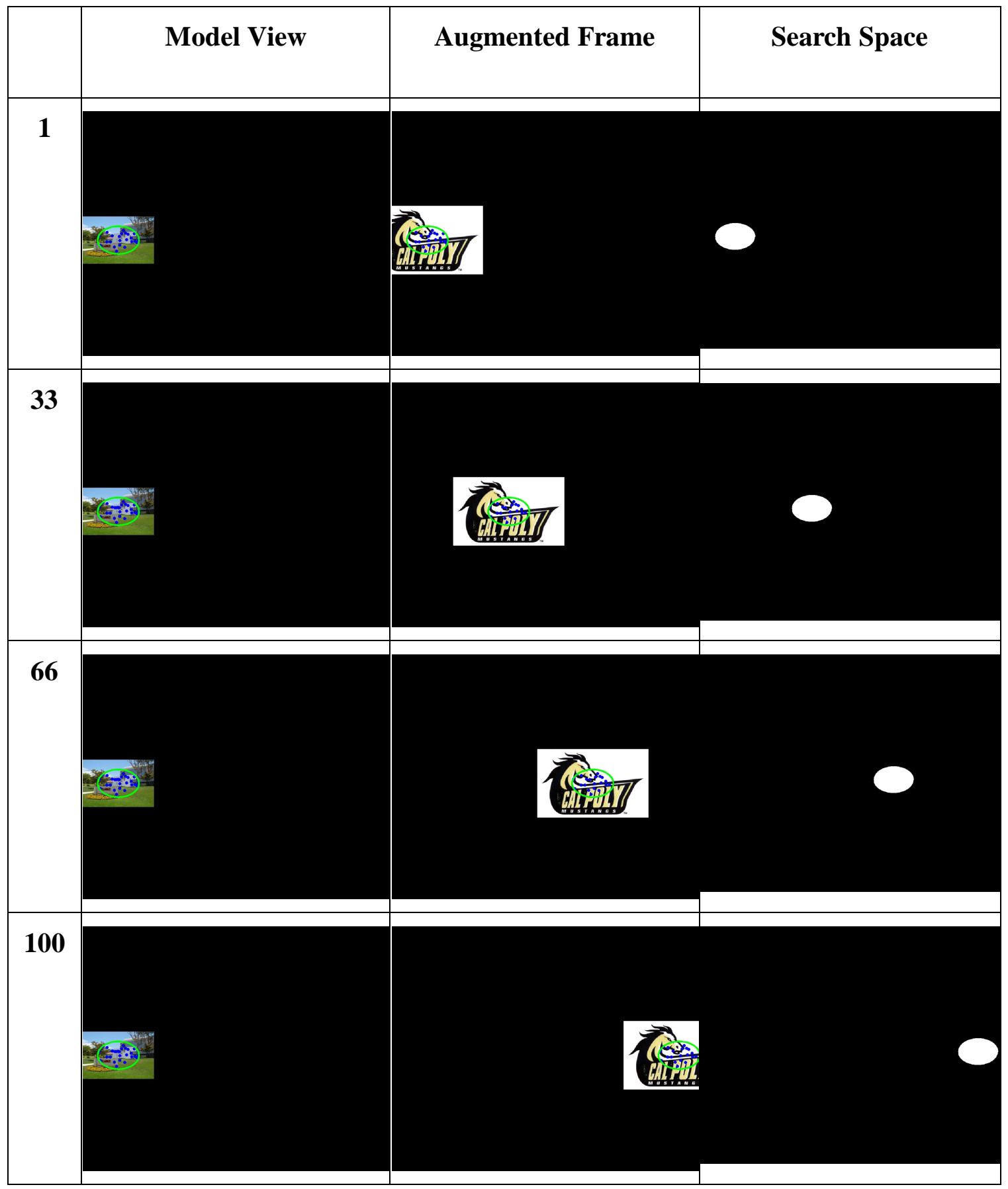

Figure 43: Translation Images, Ideal 


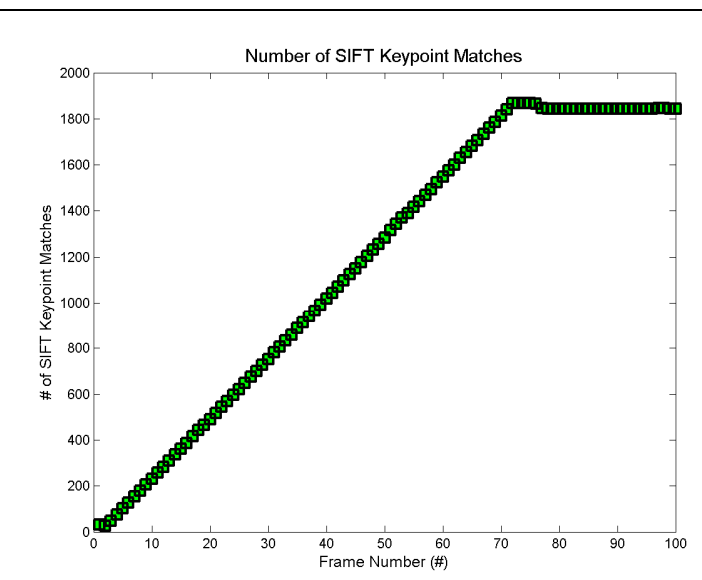

a)

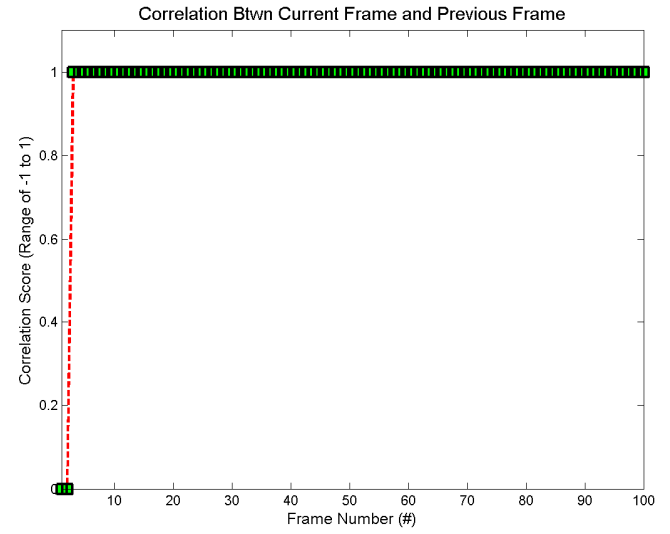

c)

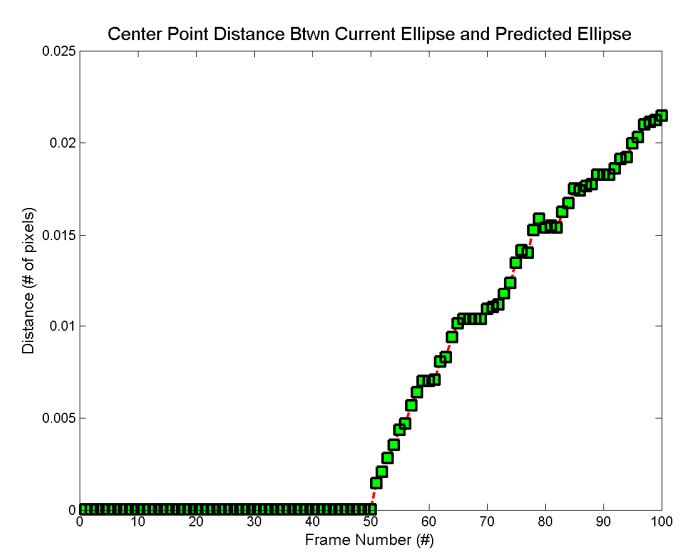

b)

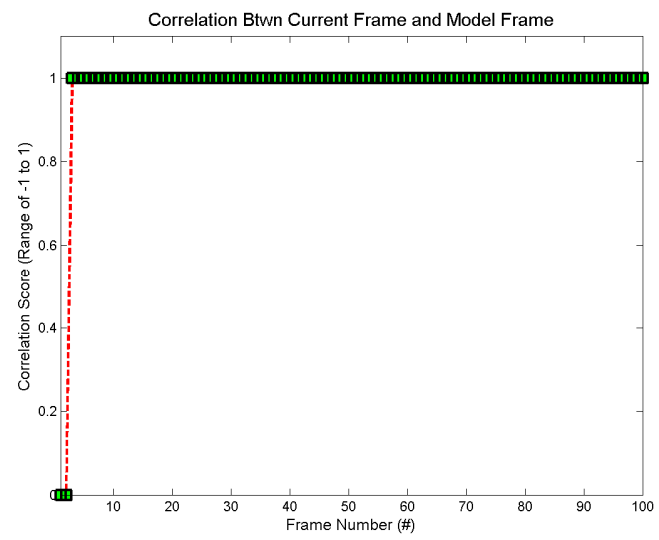

d)

Figure 44: Translation Plots Ideal, a) SIFT Keypoint Matches, b) Center Point Pixel Distances, c) Cross Correlation Btwn Current and Previous Frame, d) Cross Correlation Btwn Current and Model Frame

Table 19: Translation Data Ideal

\begin{tabular}{|r|r|r|r|}
\hline $\begin{array}{c}\text { Mean of SIFT } \\
\text { Keypoint Matches }\end{array}$ & $\begin{array}{c}\text { Mean of Center } \\
\text { Point Pixel Distances }\end{array}$ & $\begin{array}{c}\text { Mean of Cross } \\
\text { Correlation Btwn } \\
\text { Current and Previous } \\
\text { Frame }\end{array}$ & $\begin{array}{c}\text { Mean of Cross } \\
\text { Correlation Btwn } \\
\text { Current and Model } \\
\text { Frame }\end{array}$ \\
\hline 1185.85 & 0.006463 & 0.98 & 0.98 \\
\hline
\end{tabular}


Translation Test: Indoor 2, D= 6ft

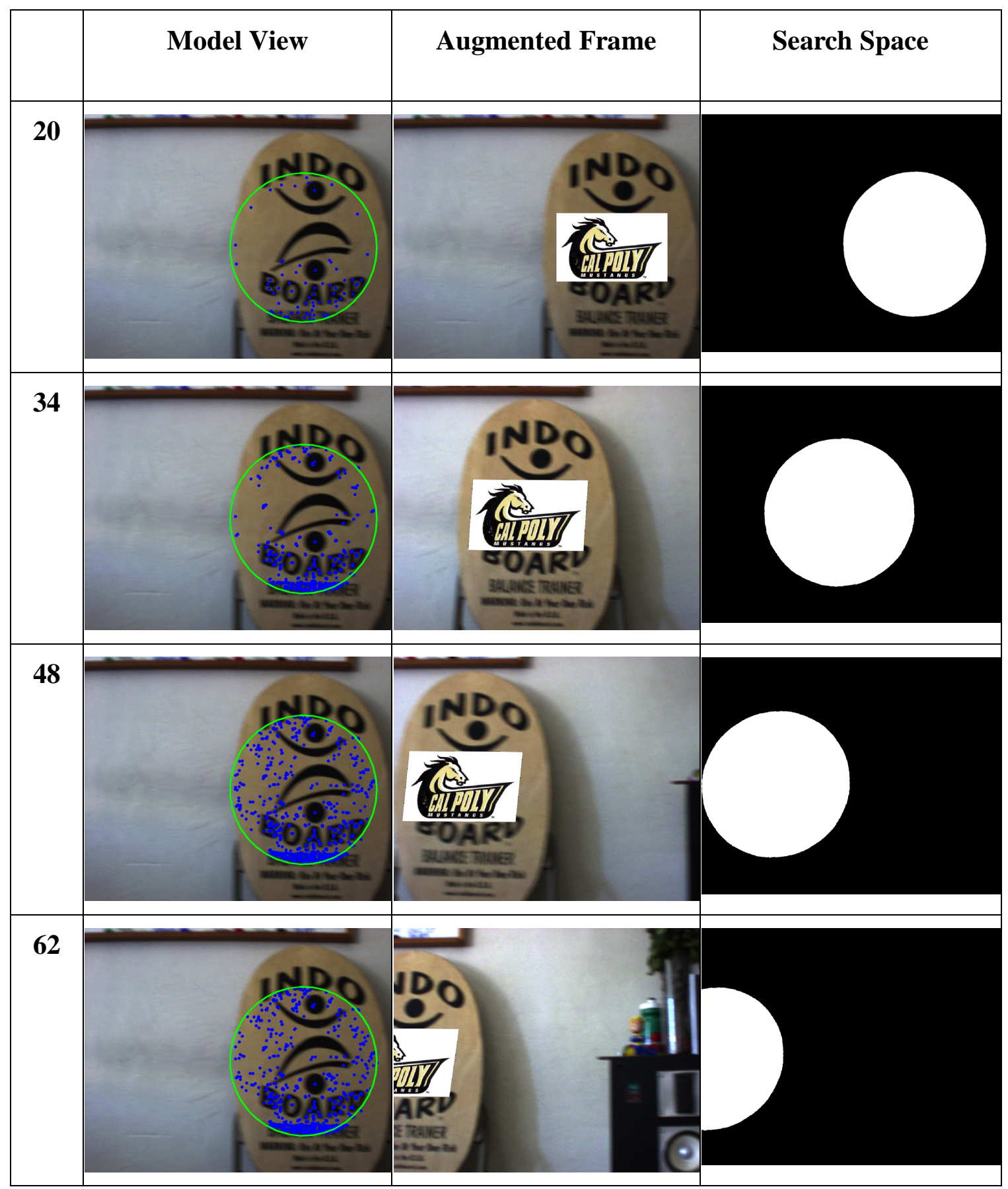

Figure 45: Translation Images, Indoor 2 


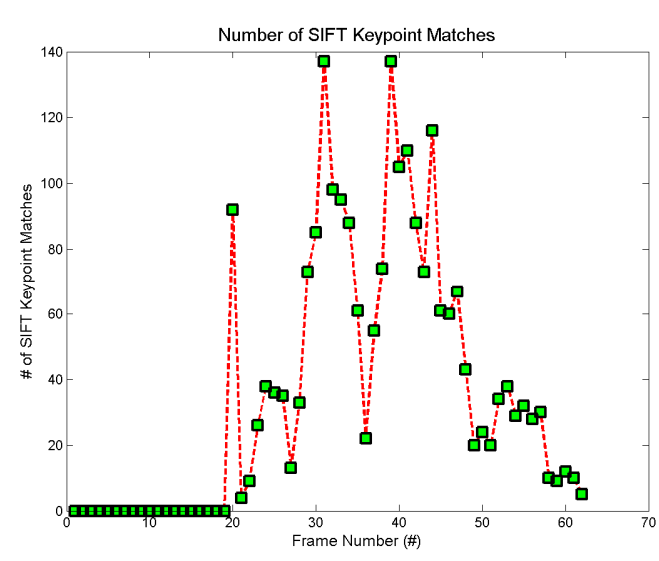

a) Frame $20=$

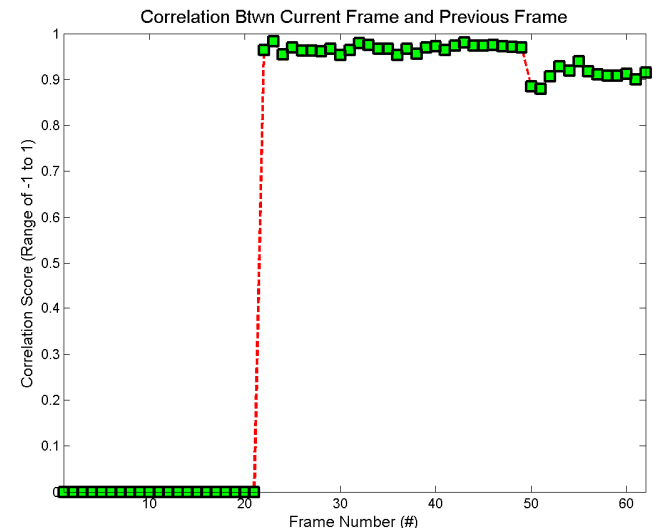

c)

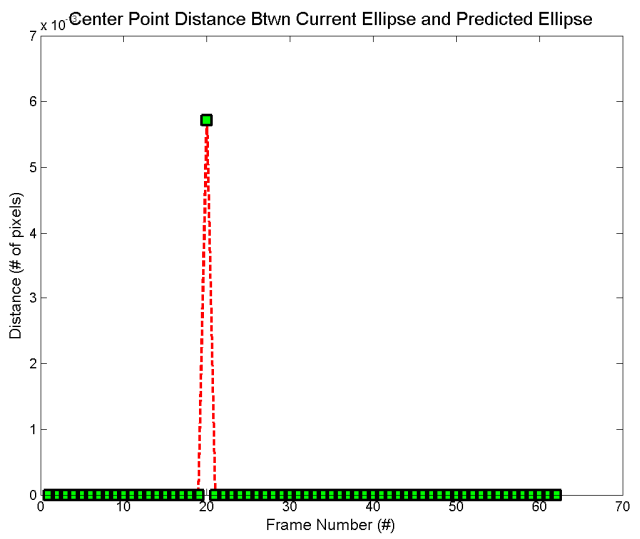

b)

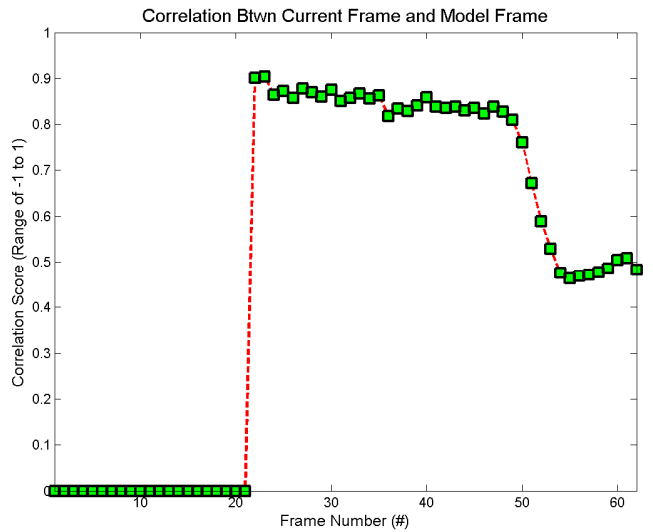

d)

Figure 46: Translation Plots Indoor 2, a) SIFT Keypoint Matches, b) Center Point Pixel Distances, c) Cross Correlation Btwn Current and Previous Frame, d) Cross Correlation Btwn Current and Model Frame

Table 20: Translation Data Indoor 2

\begin{tabular}{|r|r|r|r|}
\hline $\begin{array}{c}\text { Mean of SIFT } \\
\text { Keypoint Matches }\end{array}$ & $\begin{array}{c}\text { Mean of Center } \\
\text { Point Pixel Distances }\end{array}$ & $\begin{array}{c}\text { Mean of Cross } \\
\text { Correlation Btwn } \\
\text { Current and Previous } \\
\text { Frame }\end{array}$ & $\begin{array}{c}\text { Mean of Cross } \\
\text { Correlation Btwn } \\
\text { Current and Model } \\
\text { Frame }\end{array}$ \\
\hline 36.04839 & $9.21 \mathrm{E}-15$ & 0.627798 & 0.495391 \\
\hline
\end{tabular}


Translation Test: Outdoor $1, D=15 f t$

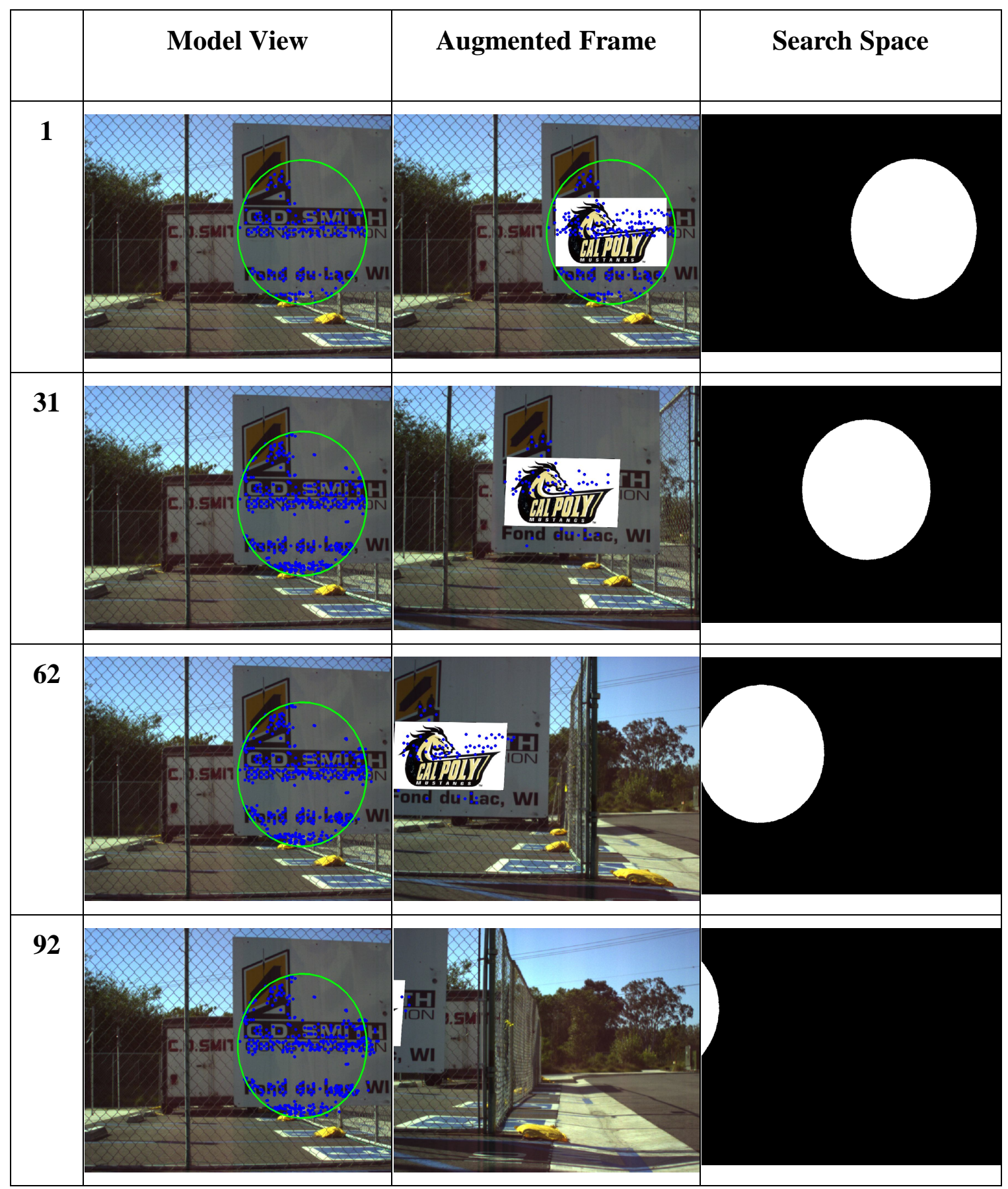

Figure 47: Translation Images, Outdoor 1 


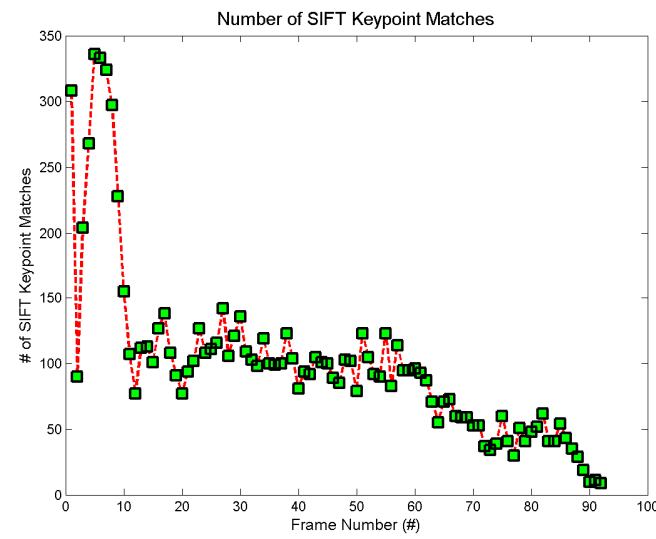

a)

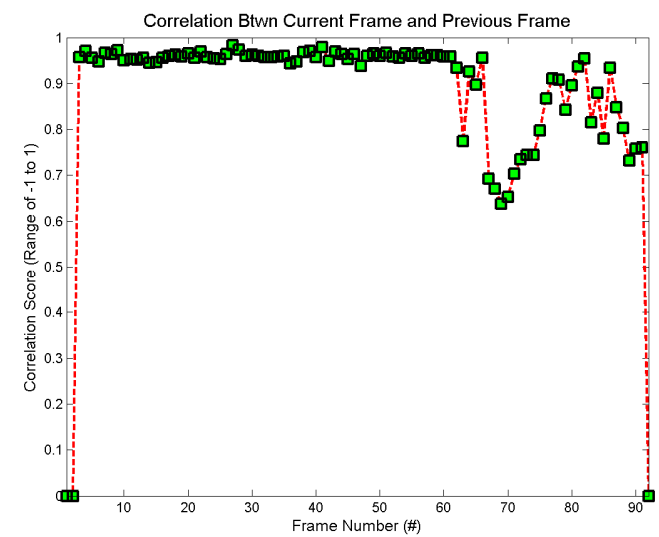

c)

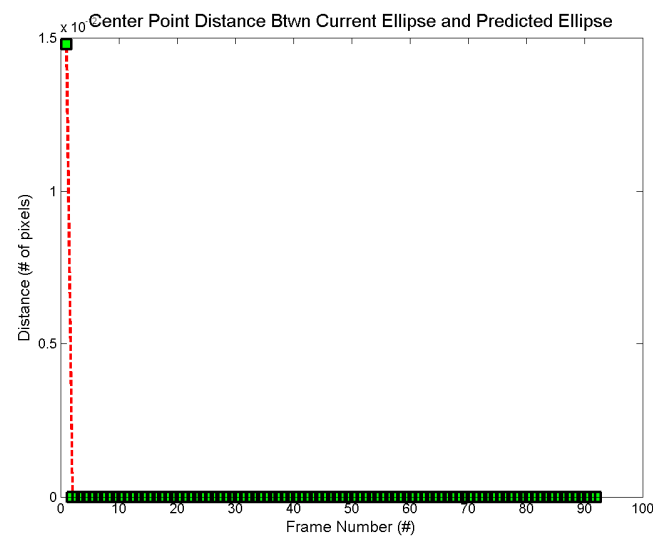

b)

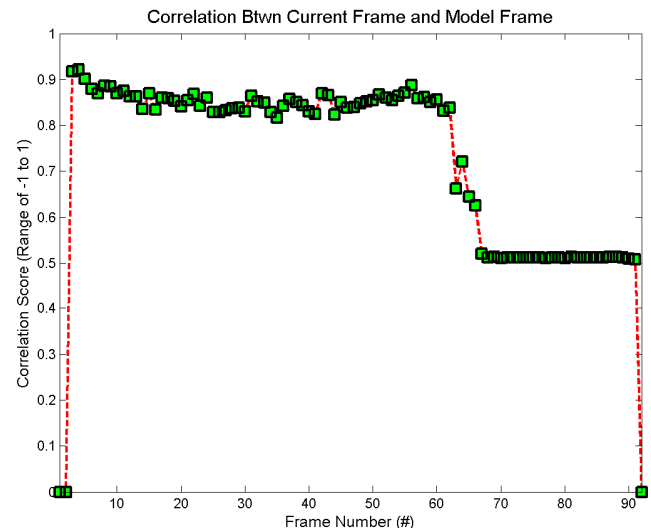

d)

Figure 48: Translation Plots Outdoor 1, a) SIFT Keypoint Matches, b) Center Point Pixel Distances, c) Cross Correlation Btwn Current and Previous Frame, d) Cross Correlation Btwn Current and Model Frame

Table 21: Translation Data Outdoor 1

\begin{tabular}{|r|r|r|r|}
\hline $\begin{array}{c}\text { Mean of SIFT } \\
\text { Keypoint Matches }\end{array}$ & $\begin{array}{c}\text { Mean of Center } \\
\text { Point Pixel Distances }\end{array}$ & $\begin{array}{c}\text { Mean of Cross } \\
\text { Correlation Btwn } \\
\text { Current and Previous } \\
\text { Frame }\end{array}$ & $\begin{array}{c}\text { Mean of Cross } \\
\text { Correlation Btwn } \\
\text { Current and Model } \\
\text { Frame }\end{array}$ \\
\hline 100.8696 & $1.61 \mathrm{E}-14$ & 0.881494 & 0.725747 \\
\hline
\end{tabular}


Translation Test: Outdoor 2, D = 12ft

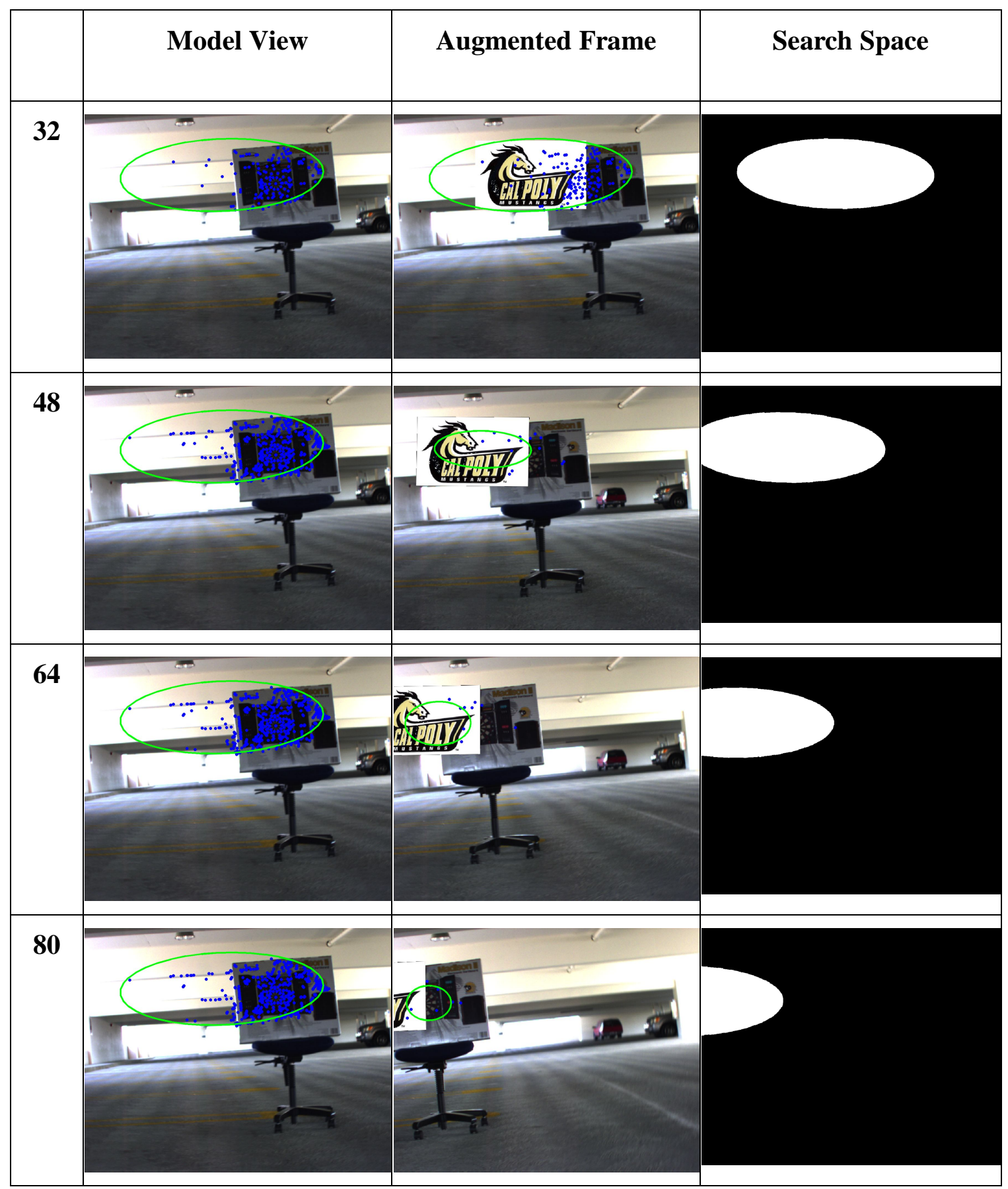

Figure 49: Translation Images, Outdoor 2 


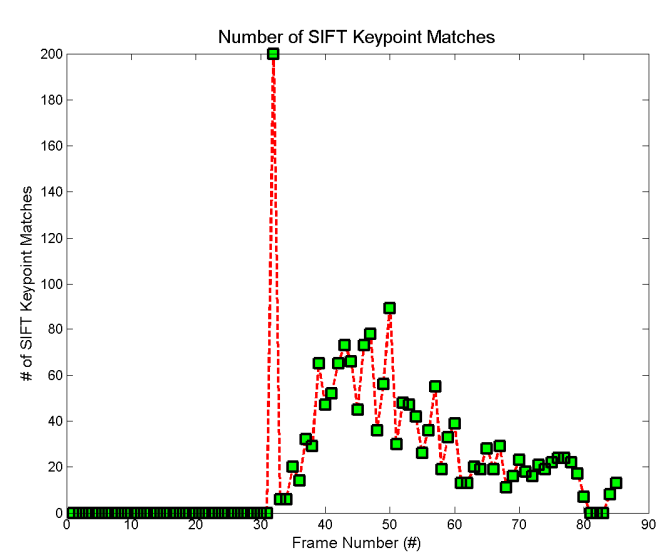

a)

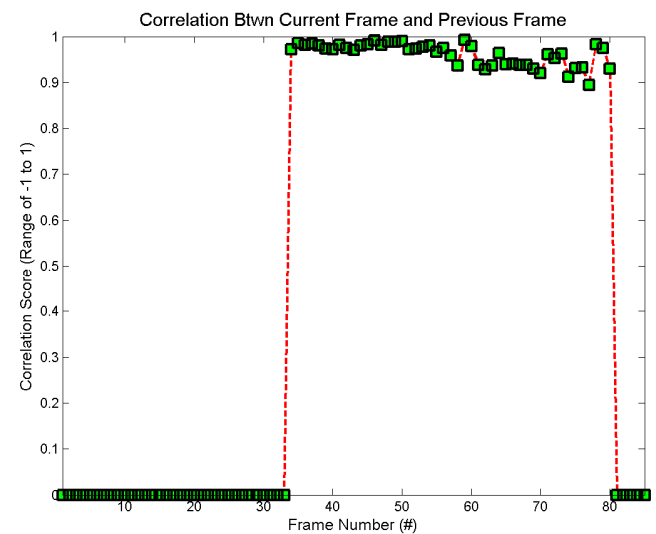

c)

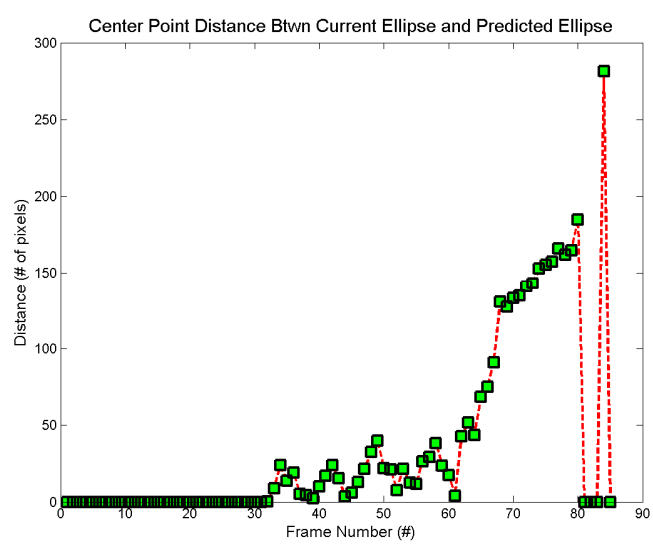

b)

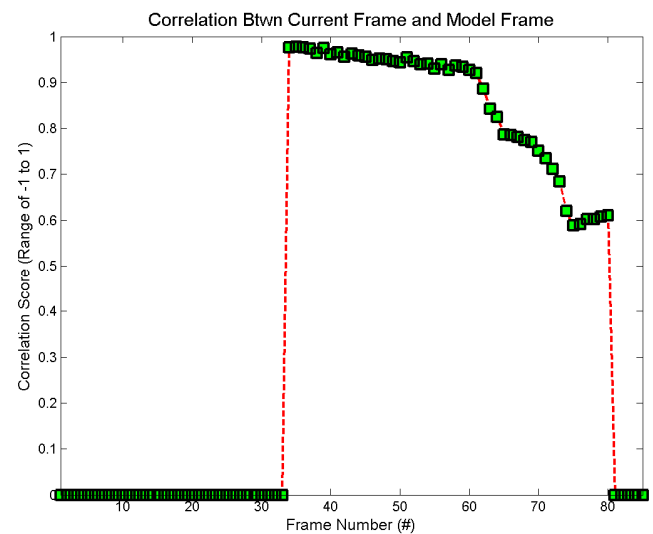

d)

Figure 50: Translation Plots Outdoor 2, a) SIFT Keypoint Matches, b) Center Point Pixel Distances, c) Cross Correlation Btwn Current and Previous Frame, d) Cross Correlation Btwn Current and Model Frame

Table 22: Translation Data Outdoor 2

\begin{tabular}{|r|r|r|r|}
\hline $\begin{array}{c}\text { Mean of SIFT } \\
\text { Keypoint Matches }\end{array}$ & $\begin{array}{c}\text { Mean of Center } \\
\text { Point Pixel Distances }\end{array}$ & $\begin{array}{c}\text { Mean of Cross } \\
\text { Correlation Btwn } \\
\text { Current and Previous } \\
\text { Frame }\end{array}$ & $\begin{array}{c}\text { Mean of Cross } \\
\text { Correlation Btwn } \\
\text { Current and Model } \\
\text { Frame }\end{array}$ \\
\hline 21.51765 & 36.50076 & 0.531731 & 0.472762 \\
\hline
\end{tabular}


Translation Test: Outdoor 3, D = 12ft

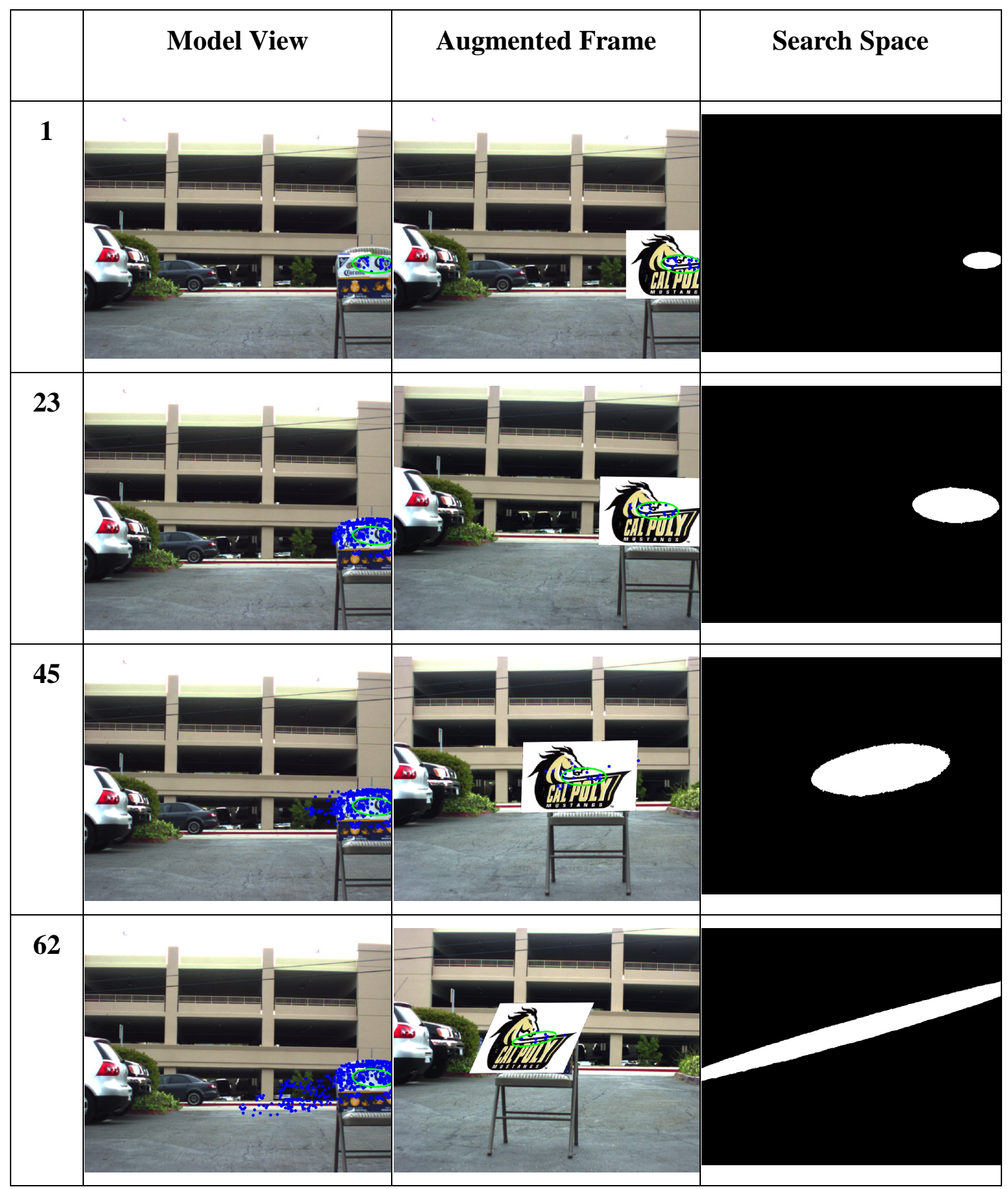

Figure 51: Translation Images, Outdoor 3 


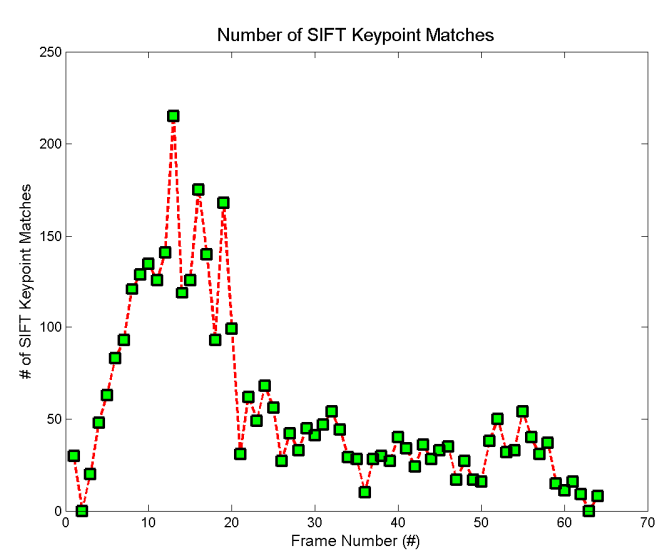

a)

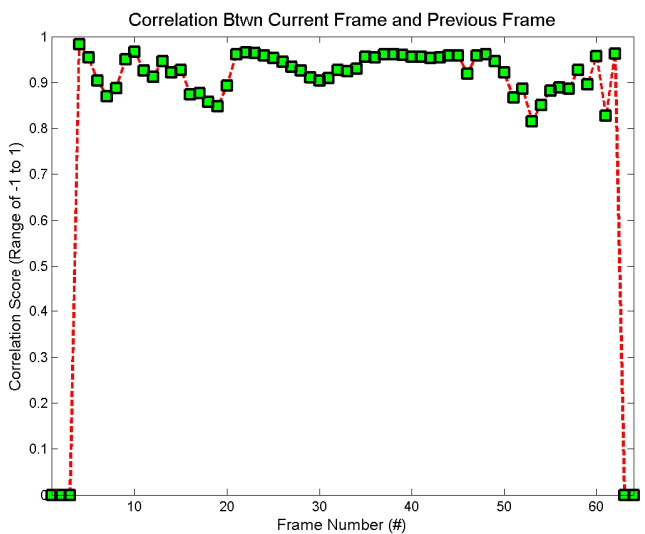

c)

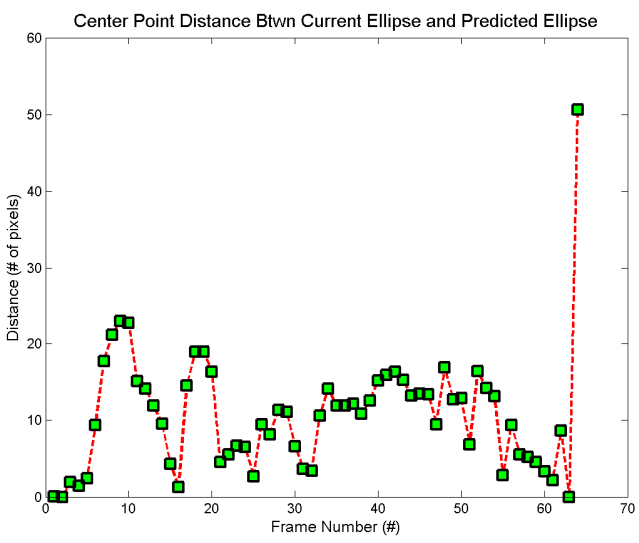

b)

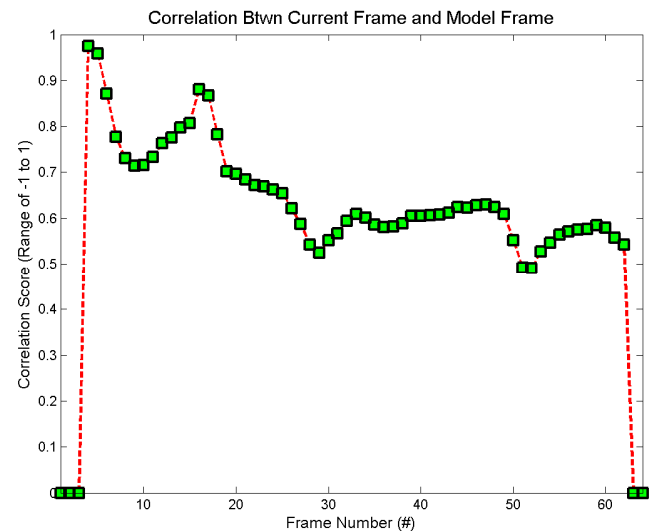

d)

Figure 52: Translation Plots Outdoor 3, a) SIFT Keypoint Matches, b) Center Point Pixel Distances, c) Cross Correlation Btwn Current and Previous Frame, d) Cross Correlation Btwn Current and Model Frame

Table 23: Translation Data Outdoor 3

\begin{tabular}{|r|r|r|r|}
\hline $\begin{array}{c}\text { Mean of SIFT } \\
\text { Keypoint Matches }\end{array}$ & $\begin{array}{c}\text { Mean of Center } \\
\text { Point Pixel Distances }\end{array}$ & $\begin{array}{c}\text { Mean of Cross } \\
\text { Correlation Btwn } \\
\text { Current and Previous } \\
\text { Frame }\end{array}$ & $\begin{array}{c}\text { Mean of Cross } \\
\text { Correlation Btwn } \\
\text { Current and Model } \\
\text { Frame }\end{array}$ \\
\hline 55.5625 & 10.7158 & 0.851296 & 0.599248 \\
\hline
\end{tabular}


Translation Test: Outdoor 4, D = 12ft

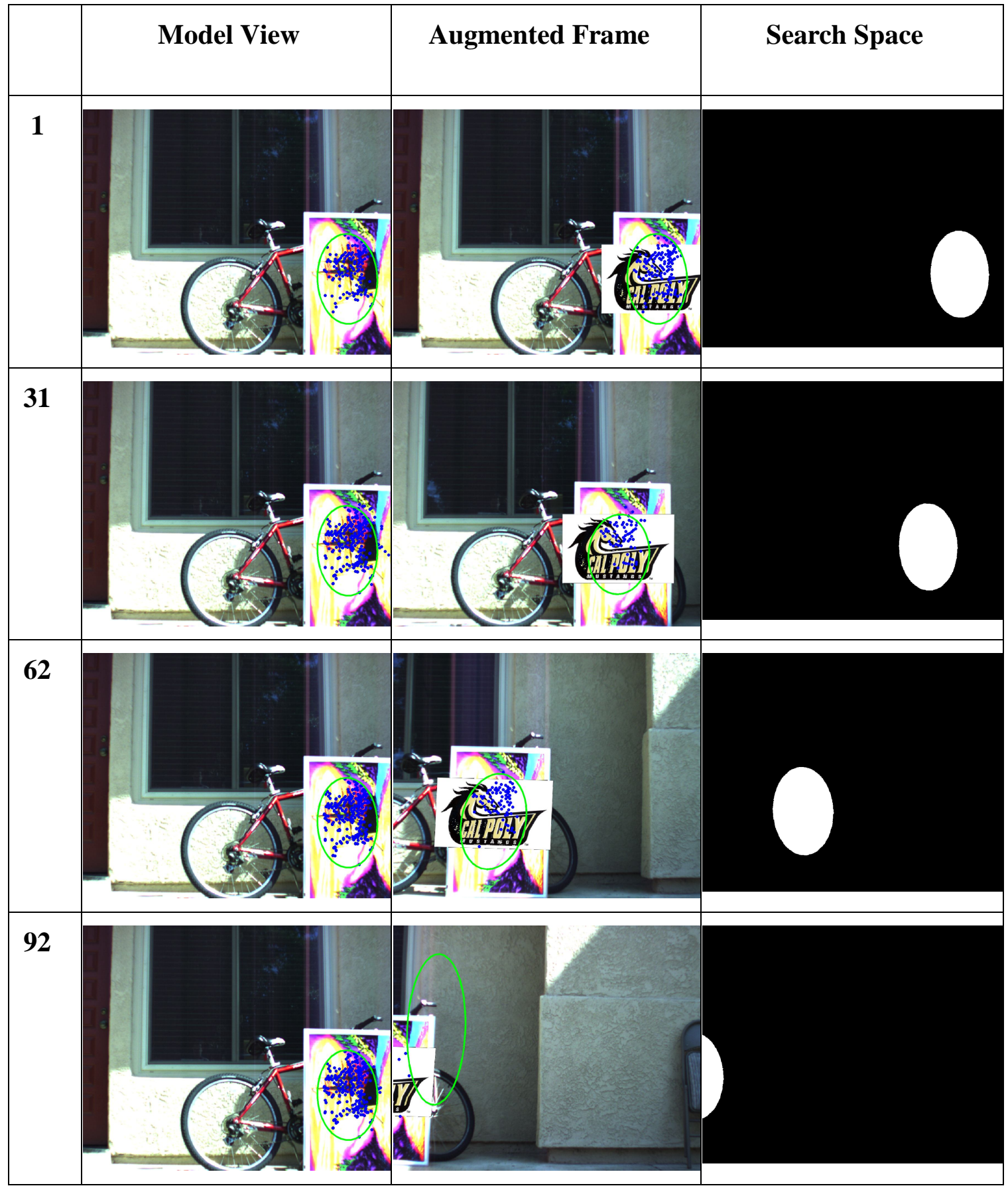

Figure 53: Translation Images, Outdoor 4 


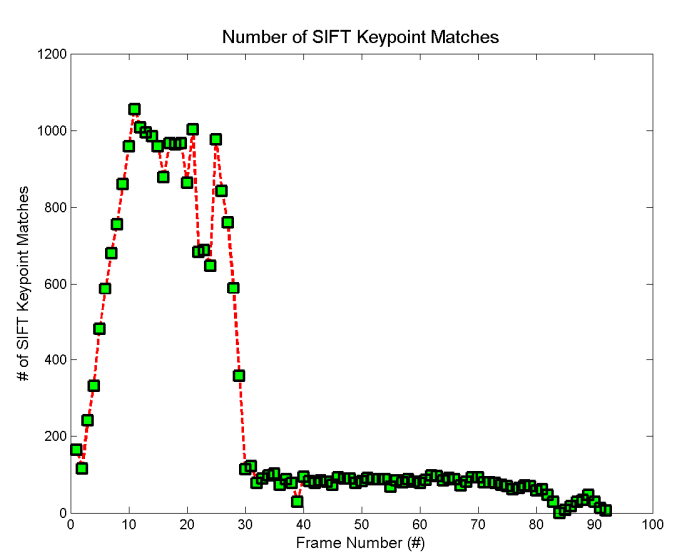

a)

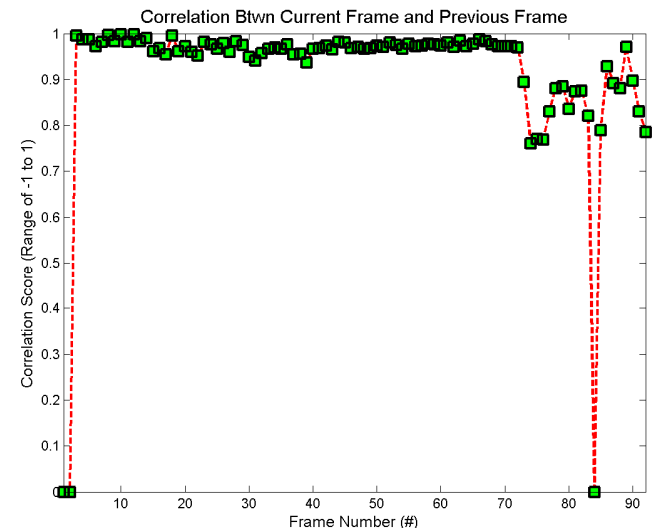

c)

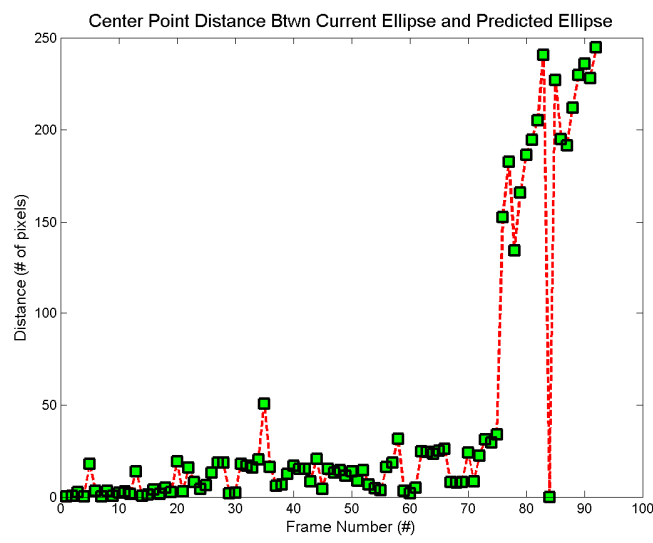

b)

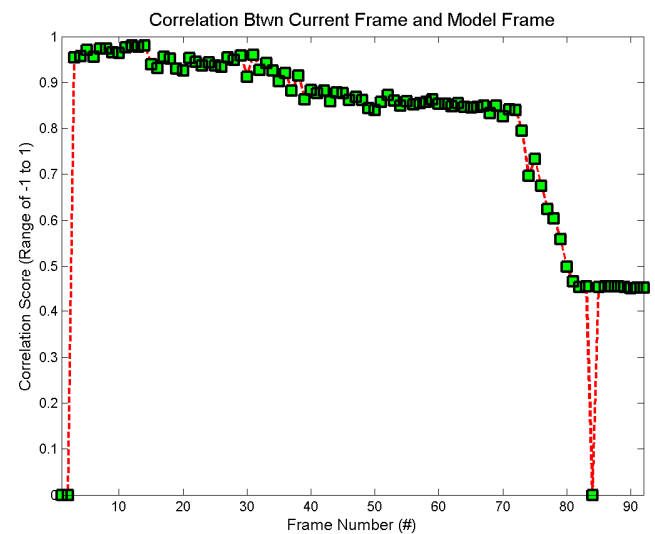

d)

Figure 54: Translation Plots Outdoor 4, a) SIFT Keypoint Matches, b) Center Point Pixel Distances, c) Cross Correlation Btwn Current and Previous Frame, d) Cross Correlation Btwn Current and Model Frame

Table 24: Translation Data Outdoor 4

\begin{tabular}{|r|r|r|r|}
\hline $\begin{array}{c}\text { Mean of SIFT } \\
\text { Keypoint Matches }\end{array}$ & $\begin{array}{c}\text { Mean of Center } \\
\text { Point Pixel Distances }\end{array}$ & $\begin{array}{c}\text { Mean of Cross } \\
\text { Correlation Btwn } \\
\text { Current and Previous } \\
\text { Frame }\end{array}$ & $\begin{array}{c}\text { Mean of Cross } \\
\text { Correlation Btwn } \\
\text { Current and Model } \\
\text { Frame }\end{array}$ \\
\hline 281.4674 & 44.82857 & 0.915956 & 0.797089 \\
\hline
\end{tabular}




\section{Rotation Tests}

Rotation Test: Ideal, D = 6ft

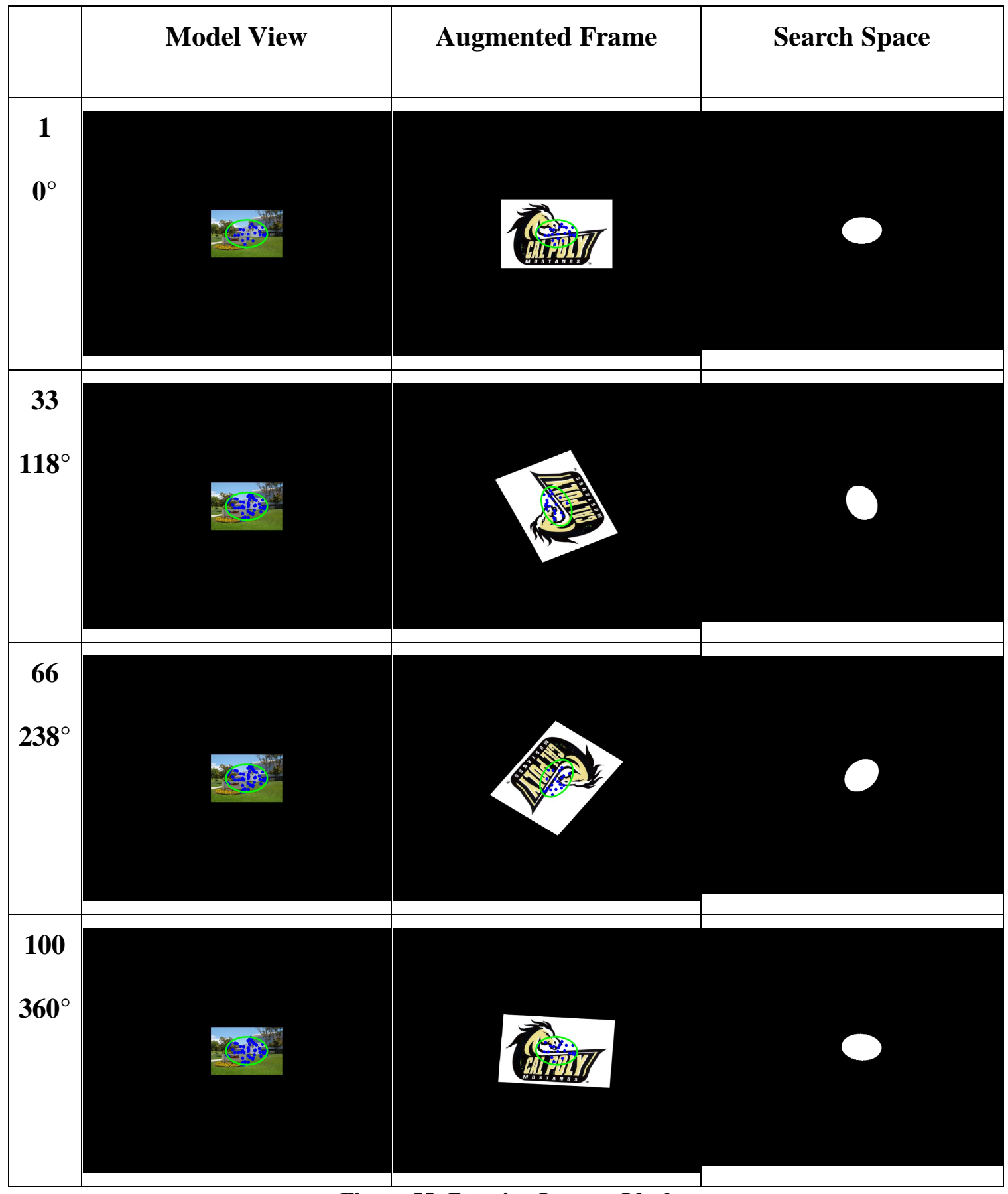

Figure 55: Rotation Images, Ideal 
Start Frame $(25)=0^{\circ}$, End Frame $(100)=360^{\circ}$, Delta $=3.6^{\circ}$ per Frame

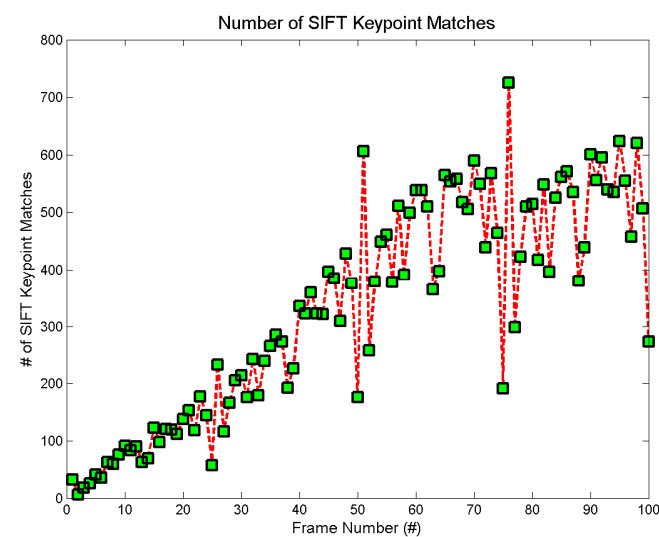

a)

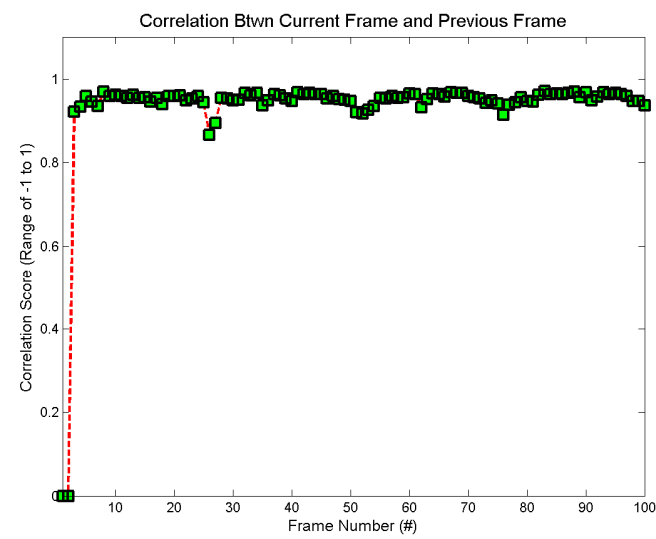

c)

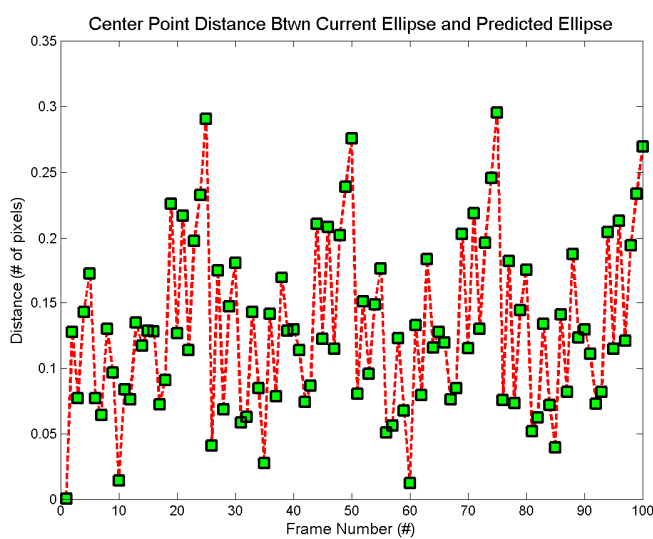

b)

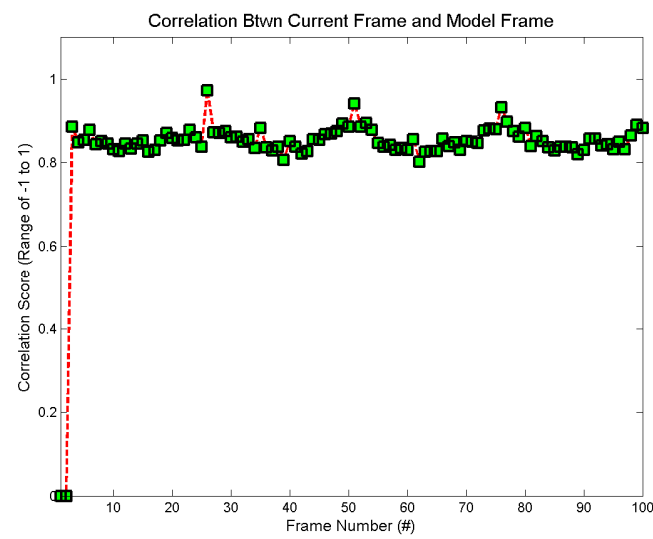

d)

Figure 56: Rotation Plots Ideal, a) SIFT Keypoint Matches, b) Center Point Pixel Distances, c) Cross Correlation Btwn Current and Previous Frame, d) Cross Correlation Btwn Current and Model

Frame

Table 25: Rotation Data Ideal

\begin{tabular}{|r|r|r|r|}
\hline $\begin{array}{c}\text { Mean of SIFT } \\
\text { Keypoint Matches }\end{array}$ & $\begin{array}{c}\text { Mean of Center } \\
\text { Point Pixel Distances }\end{array}$ & $\begin{array}{c}\text { Mean of Cross } \\
\text { Correlation Btwn } \\
\text { Current and Previous } \\
\text { Frame }\end{array}$ & $\begin{array}{c}\text { Mean of Cross } \\
\text { Correlation Btwn } \\
\text { Current and Model } \\
\text { Frame }\end{array}$ \\
\hline 333.6 & 0.130074 & 0.933787 & 0.837808 \\
\hline
\end{tabular}


Rotation Test: Indoor 2, D = 6ft

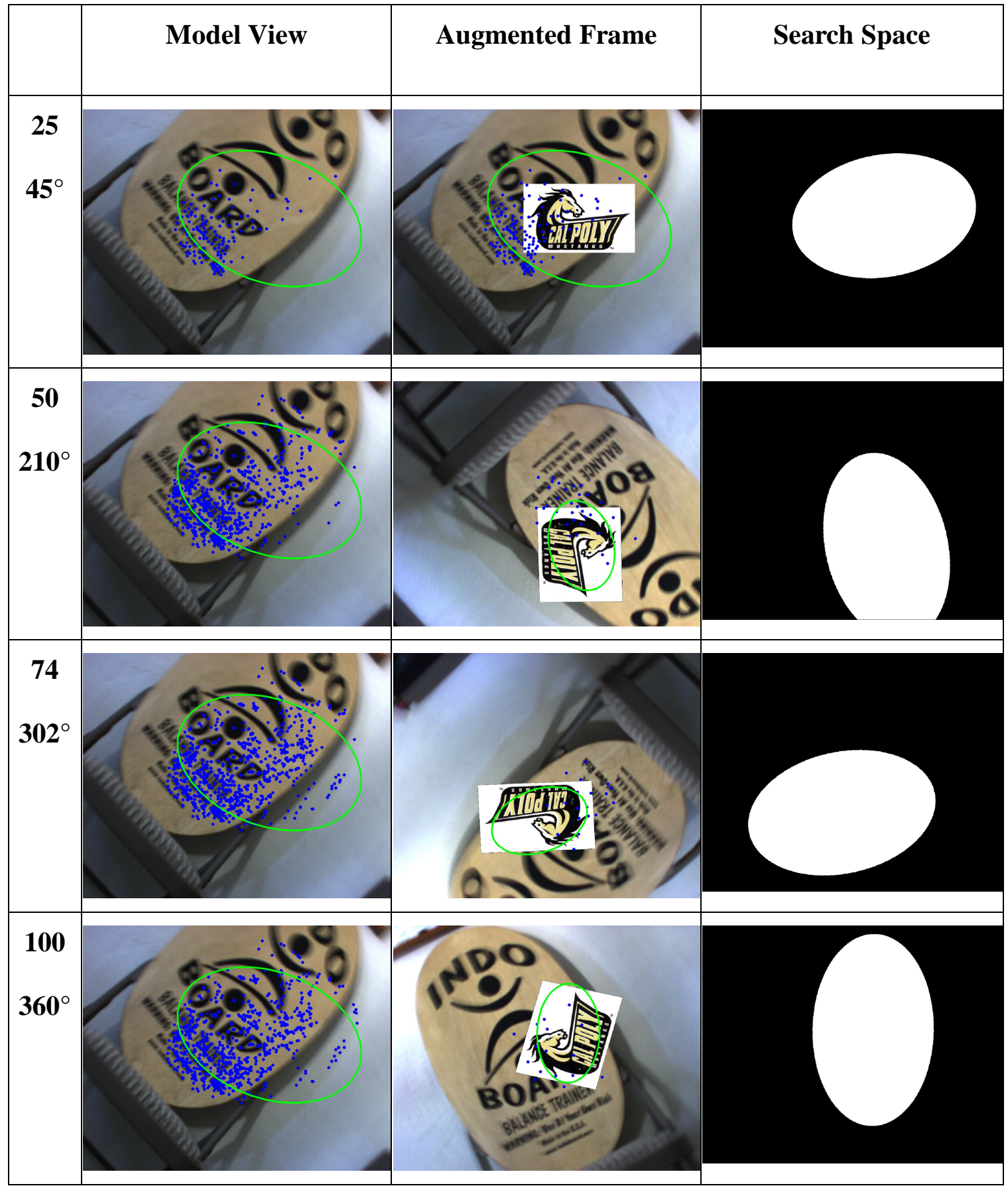

Figure 57: Rotation Images, Indoor 2 
Start Frame $(25)=45^{\circ}$, End Frame $(100)=360^{\circ}$, Delta $=4.2^{\circ}$ per Frame

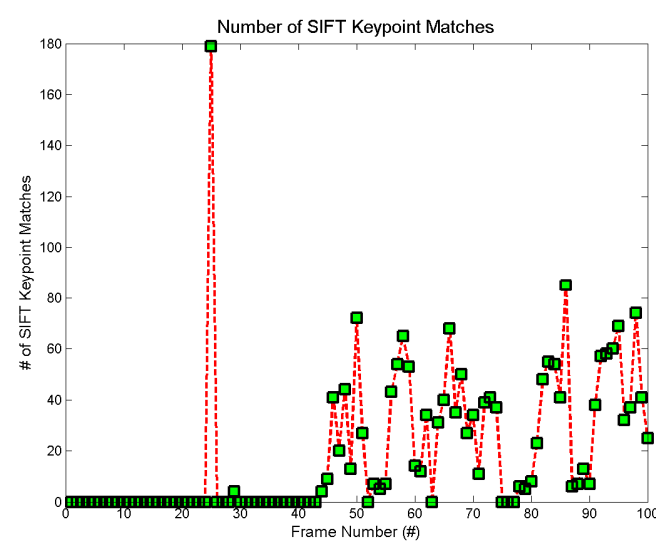

a)

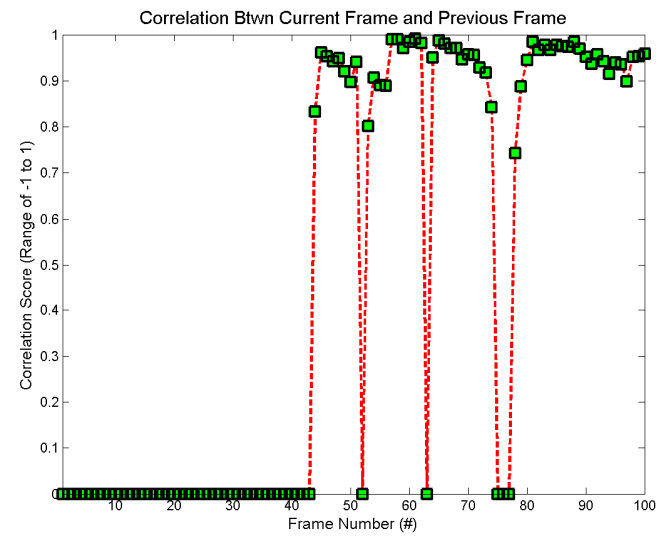

c)

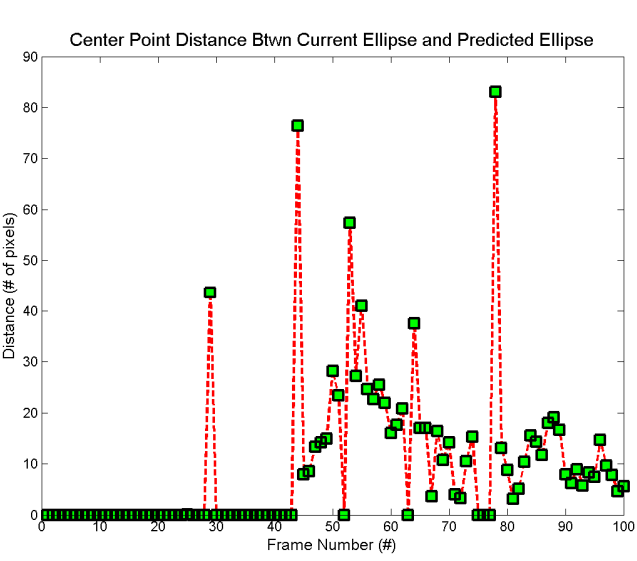

b)

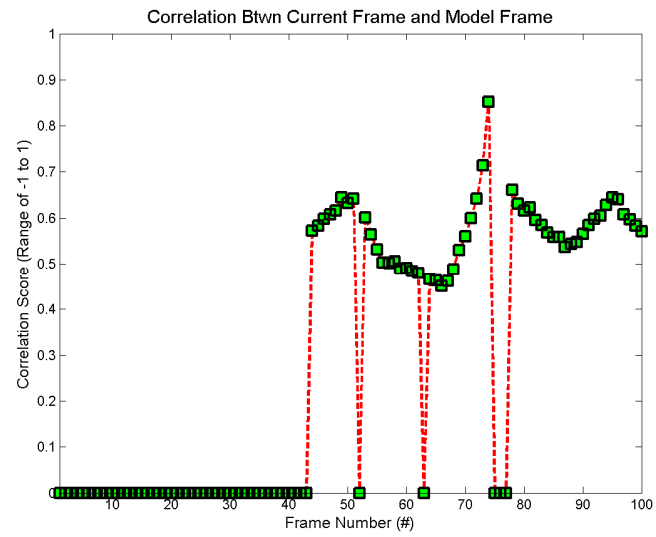

d)

Figure 58: Rotation Plots Indoor 2, a) SIFT Keypoint Matches, b) Center Point Pixel Distances, c) Cross Correlation Btwn Current and Previous Frame, d) Cross Correlation Btwn Current and Model Frame

Table 26: Rotation Data Indoor 2

\begin{tabular}{|c|c|c|c|}
\hline $\begin{array}{c}\text { Mean of SIFT } \\
\text { Keypoint Matches }\end{array}$ & $\begin{array}{c}\text { Mean of Center } \\
\text { Point Pixel Distances }\end{array}$ & $\begin{array}{c}\text { Mean of Cross } \\
\text { Correlation Btwn } \\
\text { Current and Previous } \\
\text { Frame }\end{array}$ & $\begin{array}{c}\text { Mean of Cross } \\
\text { Correlation Btwn } \\
\text { Current and Model } \\
\text { Frame }\end{array}$ \\
\hline 19.69 & 9.599946 & 0.488831 & 0.299184 \\
\hline
\end{tabular}


Rotation Test: Indoor 2, D = 12ft

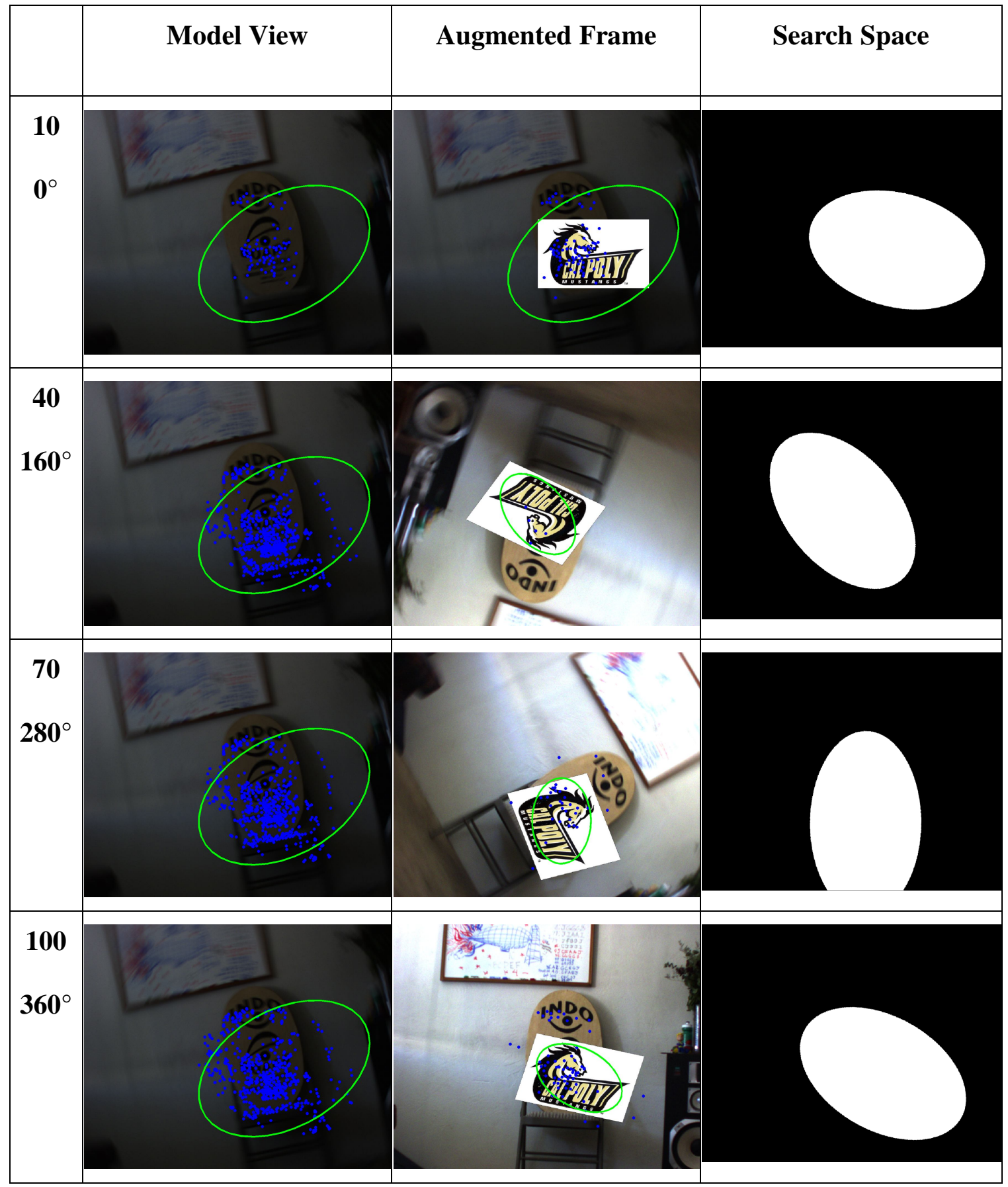

Figure 59: Rotation Images, Indoor 2 
Start Frame $(10)=0^{\circ}$, End Frame $(100)=360^{\circ}$, Delta $=4^{\circ}$ per Frame

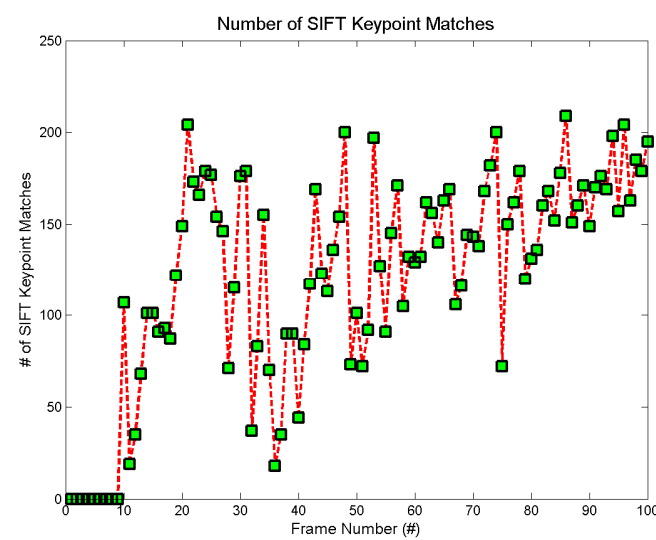

a)

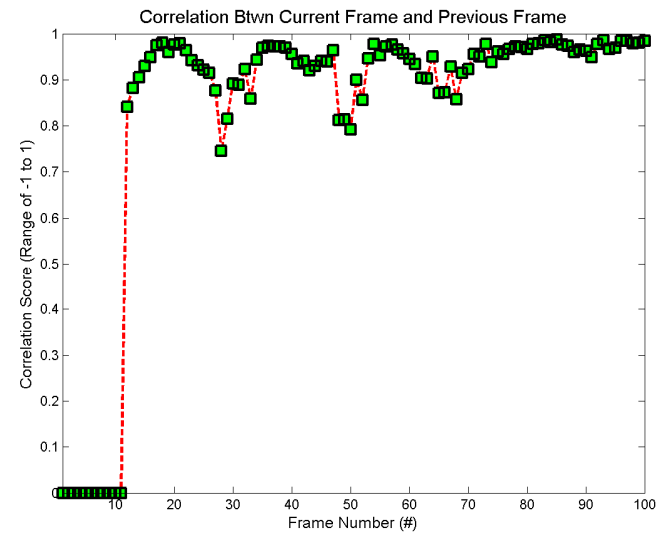

c)

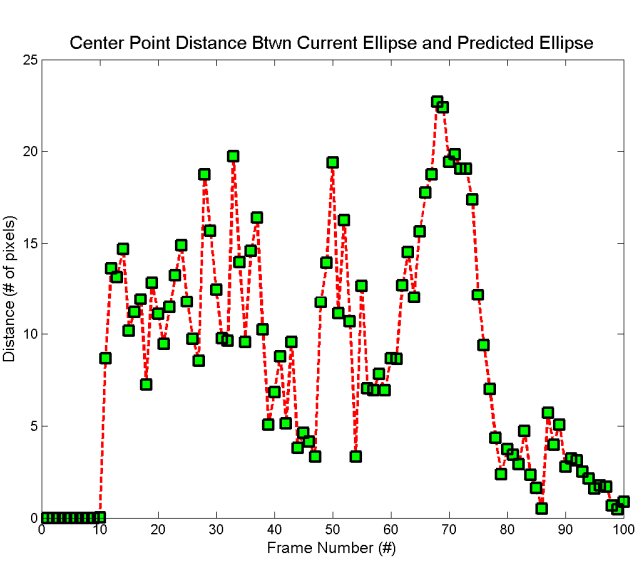

b)

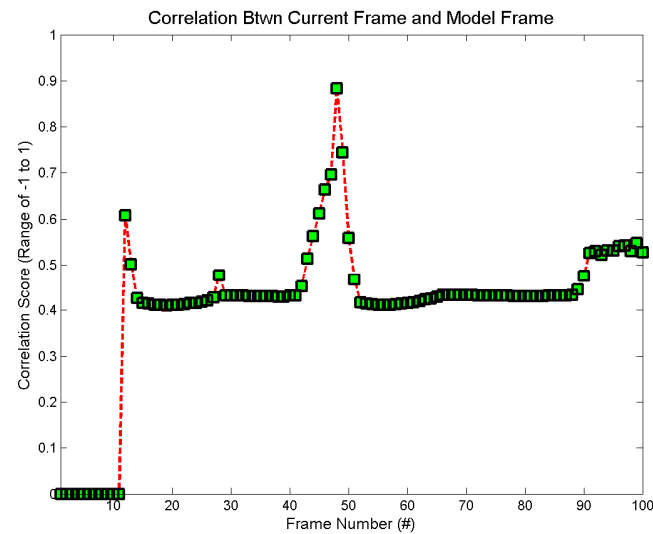

d)

Figure 60: Rotation Plots Indoor 2, a) SIFT Keypoint Matches, b) Center Point Pixel Distances, c) Cross Correlation Btwn Current and Previous Frame, d) Cross Correlation Btwn Current and Model Frame

Table 27: Rotation Data Indoor 2

\begin{tabular}{|c|c|c|c|}
\hline $\begin{array}{c}\text { Mean of SIFT } \\
\text { Keypoint Matches }\end{array}$ & $\begin{array}{c}\text { Mean of Center } \\
\text { Point Pixel Distances }\end{array}$ & $\begin{array}{c}\text { Mean of Cross } \\
\text { Correlation Btwn } \\
\text { Current and Previous } \\
\text { Frame }\end{array}$ & $\begin{array}{c}\text { Mean of Cross } \\
\text { Correlation Btwn } \\
\text { Current and Model } \\
\text { Frame }\end{array}$ \\
\hline 8.620545 & 122.29 & 0.834187 & 0.41181 \\
\hline
\end{tabular}


Rotation Test: Outdoor 1, D = 12ft

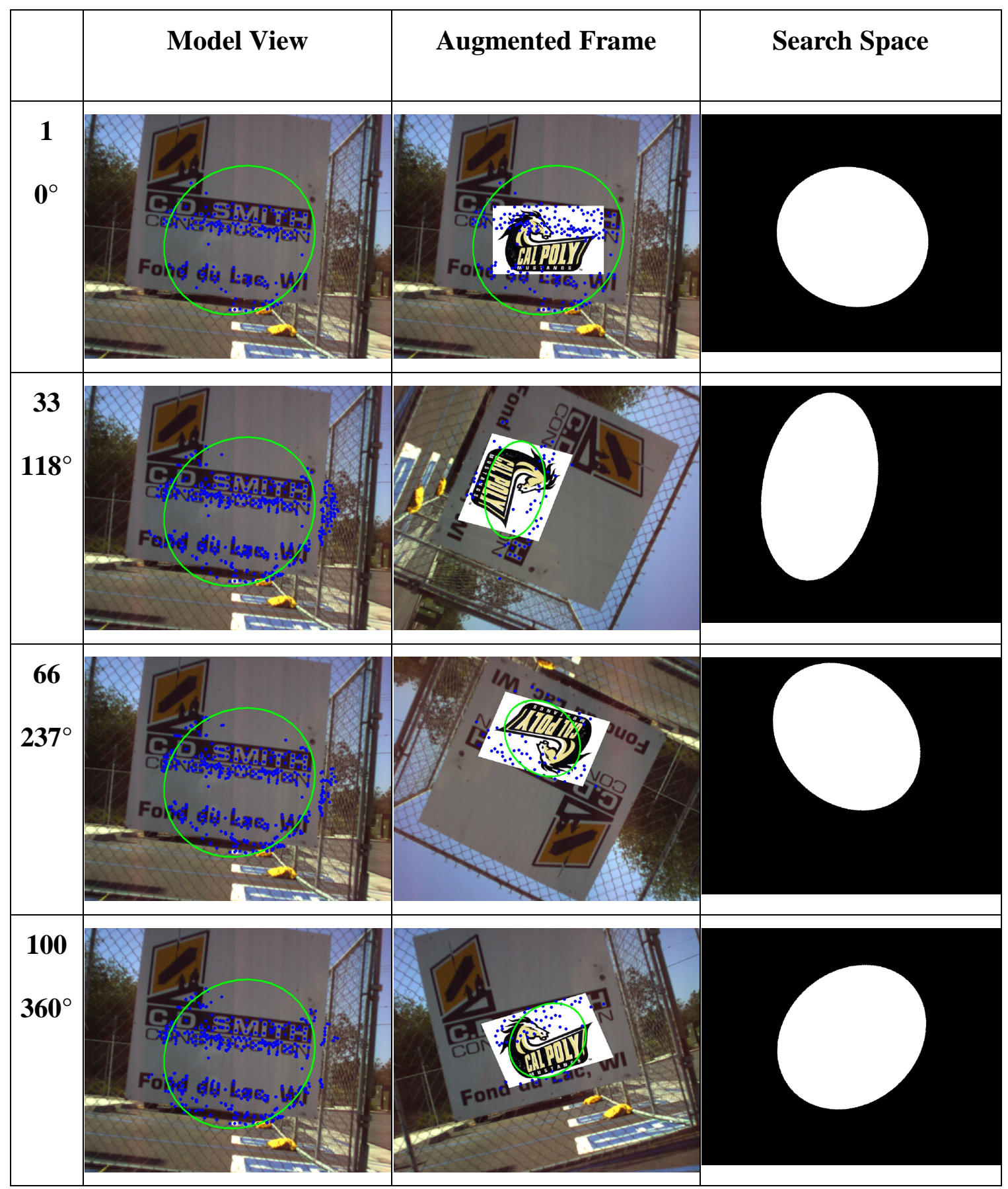

Figure 61: Rotation Images, Outdoor 1 
Start Frame $(1)=0^{\circ}$, End Frame $(100)=360^{\circ}$, Delta $=3.6^{\circ}$ per Frame

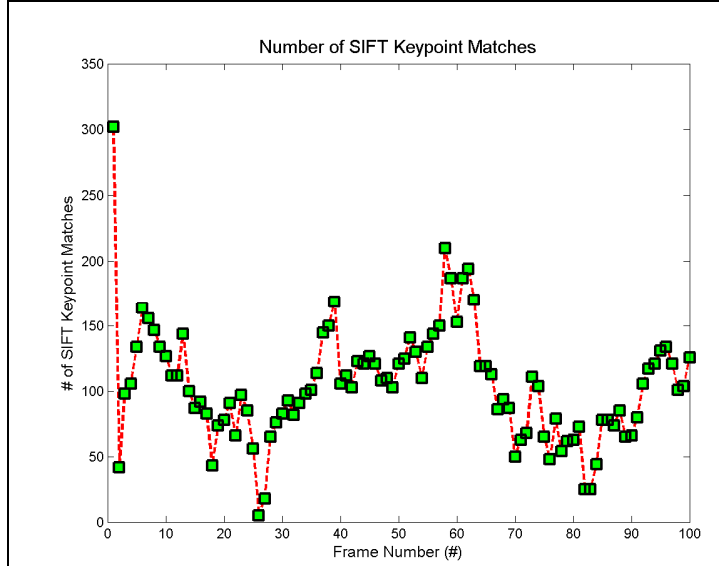

a)

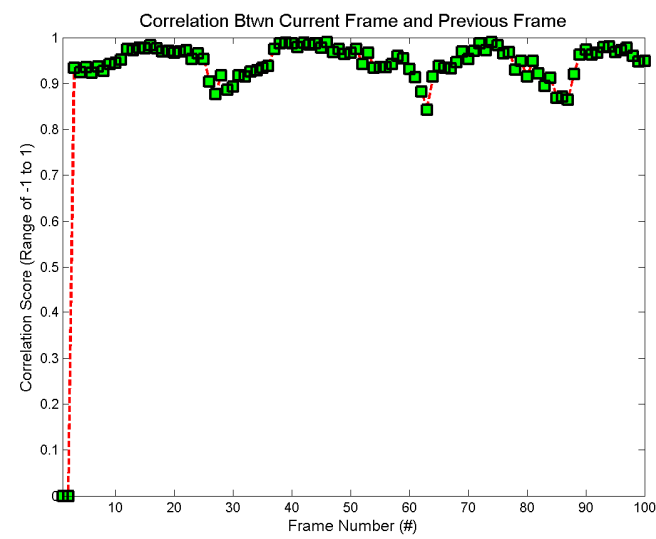

c)

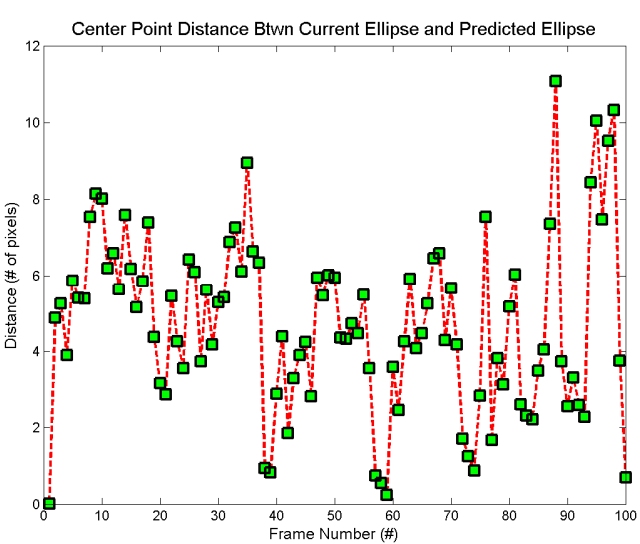

b)

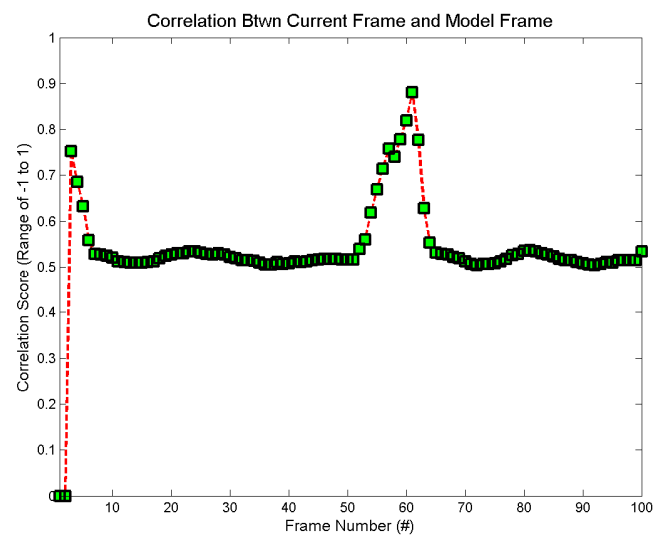

d)

Figure 62: Rotation Plots Outdoor 1, a) SIFT Keypoint Matches, b) Center Point Pixel Distances, c) Cross Correlation Btwn Current and Previous Frame, d) Cross Correlation Btwn Current and Model Frame

Table 28: Rotation Data Outdoor 1

\begin{tabular}{|r|r|r|r|}
\hline $\begin{array}{c}\text { Mean of SIFT } \\
\text { Keypoint Matches }\end{array}$ & $\begin{array}{c}\text { Mean of Center } \\
\text { Point Pixel Distances }\end{array}$ & $\begin{array}{c}\text { Mean of Cross } \\
\text { Correlation Btwn } \\
\text { Current and Previous } \\
\text { Frame }\end{array}$ & $\begin{array}{c}\text { Mean of Cross } \\
\text { Correlation Btwn } \\
\text { Current and Model } \\
\text { Frame }\end{array}$ \\
\hline 104.49 & 4.739818 & 0.92876 & 0.536275 \\
\hline
\end{tabular}


Rotation Test: Outdoor 2, D = 12ft

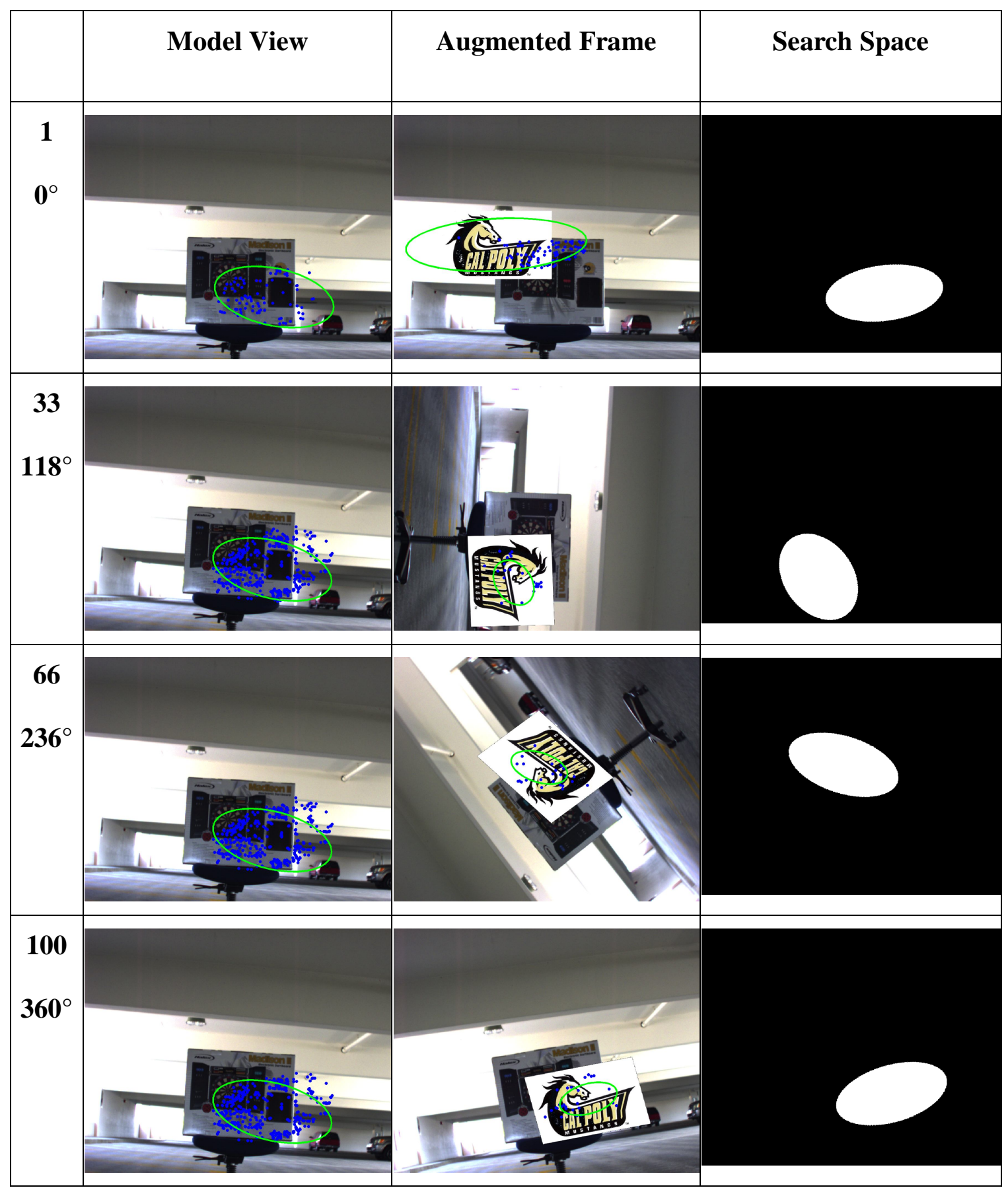

Figure 63: Rotation Images, Outdoor 2 
Start Frame $(1)=0^{\circ}$, End Frame $(100)=360^{\circ}$, Delta $=3.6^{\circ}$ per Frame

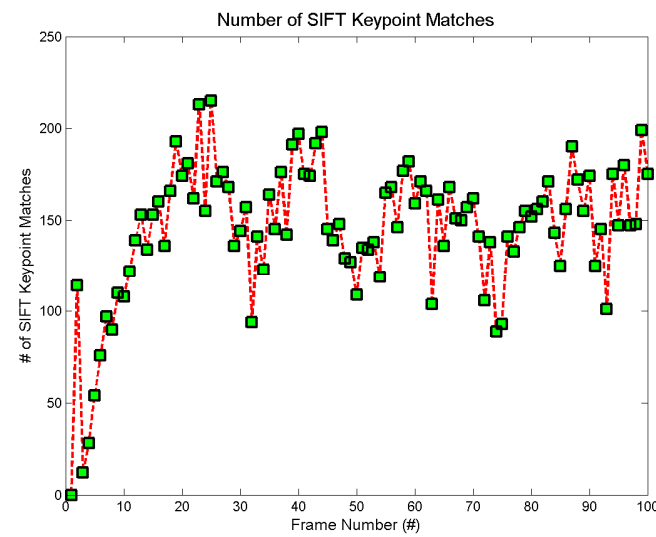

a)

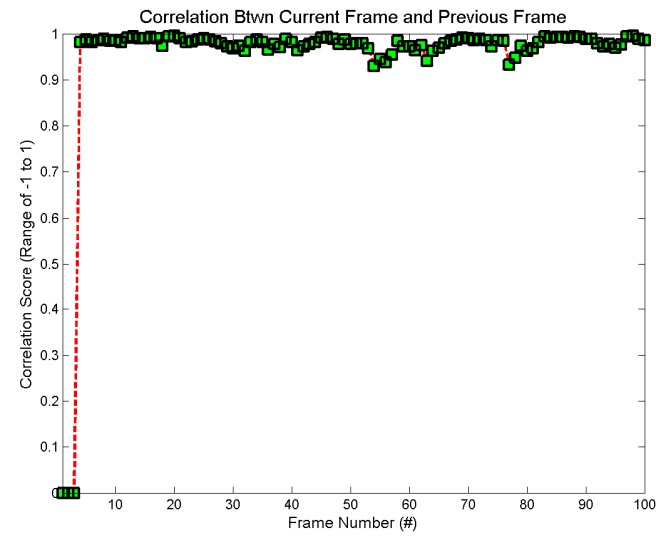

c)

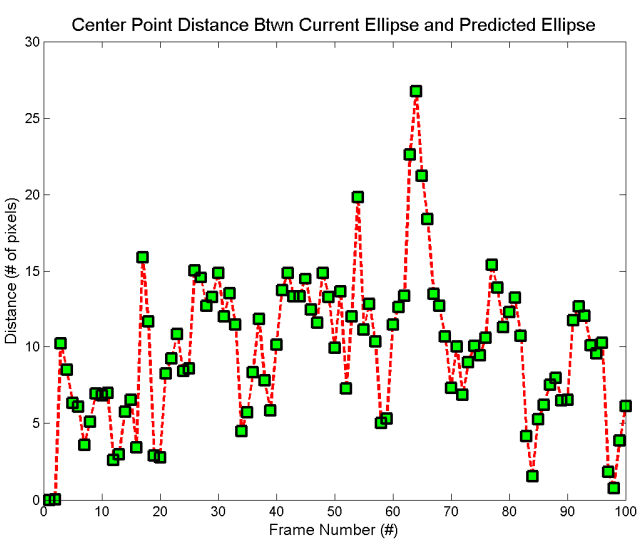

b)

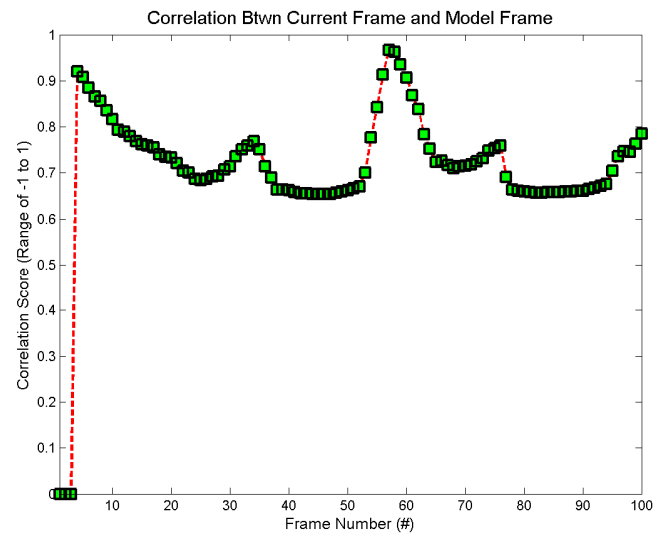

d)

Figure 64: Rotation Plots Outdoor 2, a) SIFT Keypoint Matches, b) Center Point Pixel Distances, c) Cross Correlation Btwn Current and Previous Frame, d) Cross Correlation Btwn Current and Model Frame

Table 29: Rotation Data Outdoor 2

\begin{tabular}{|c|c|c|c|}
\hline $\begin{array}{c}\text { Mean of SIFT } \\
\text { Keypoint Matches }\end{array}$ & $\begin{array}{c}\text { Mean of Center } \\
\text { Point Pixel Distances }\end{array}$ & $\begin{array}{c}\text { Mean of Cross } \\
\text { Correlation Btwn } \\
\text { Current and Previous } \\
\text { Frame }\end{array}$ & $\begin{array}{c}\text { Mean of Cross } \\
\text { Correlation Btwn } \\
\text { Current and Model } \\
\text { Frame }\end{array}$ \\
\hline 144.93 & 9.778651 & 0.949811 & 0.711703 \\
\hline
\end{tabular}


Rotation Test: Outdoor 3, D = 12ft

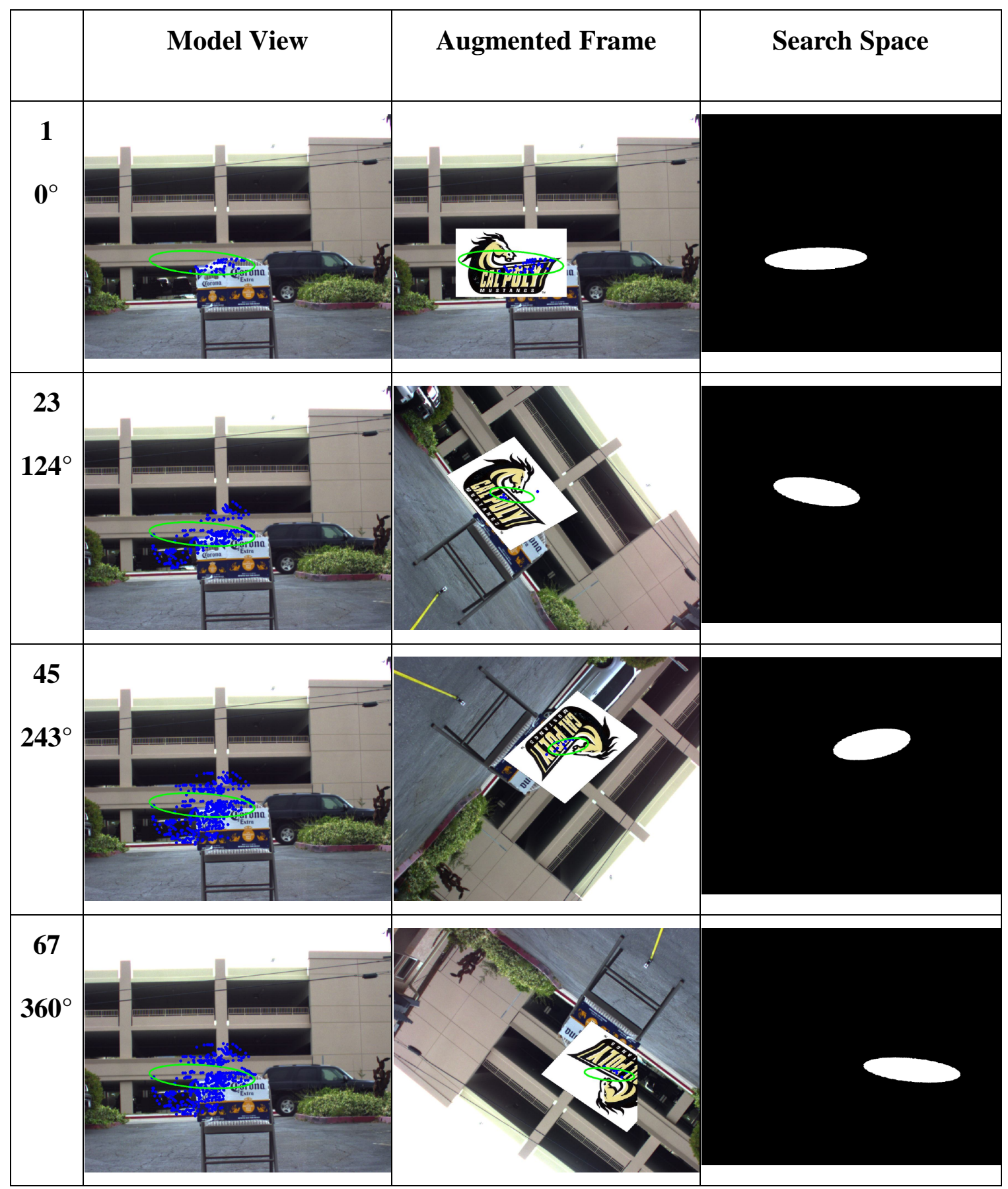

Figure 65: Rotation Images, Outdoor 3 
Start Frame $(1)=0^{\circ}$, End Frame $(67)=360^{\circ}$, Delta $=5.4^{\circ}$ per Frame

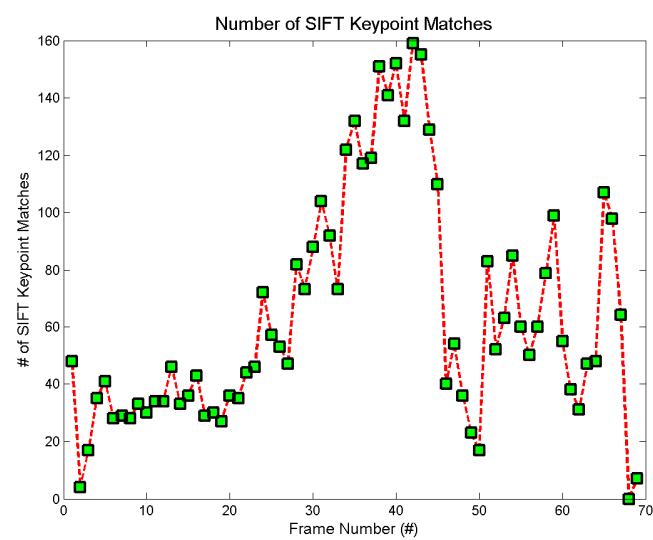

a)

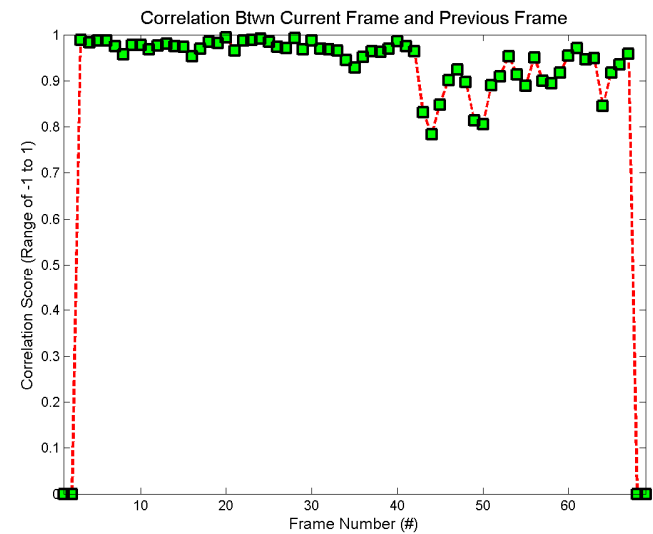

c)

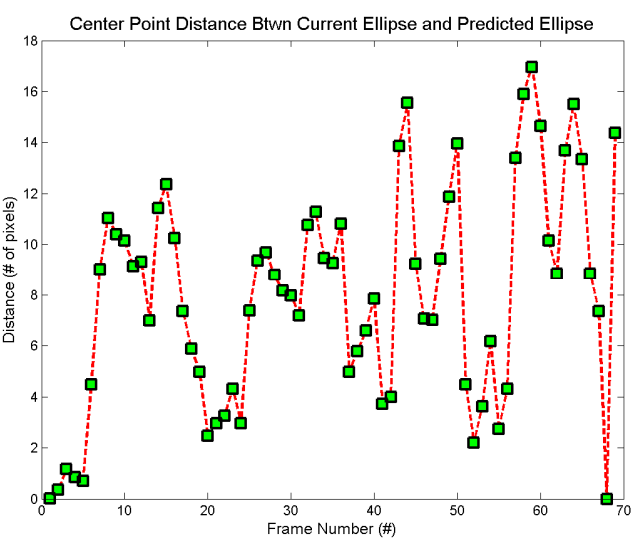

b)

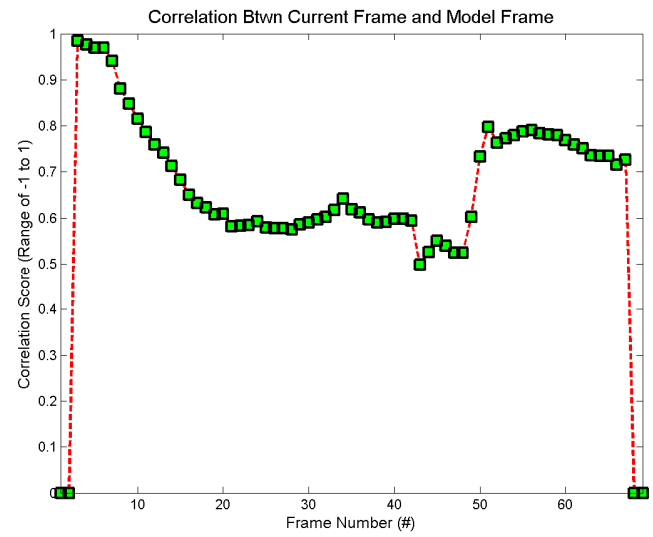

d)

Figure 66: Rotation Plots Outdoor 3, a) SIFT Keypoint Matches, b) Center Point Pixel Distances, c) Cross Correlation Btwn Current and Previous Frame, d) Cross Correlation Btwn Current and Model Frame

Table 30: Rotation Data Outdoor 3

\begin{tabular}{|c|c|c|c|}
\hline $\begin{array}{c}\text { Mean of SIFT } \\
\text { Keypoint Matches }\end{array}$ & $\begin{array}{c}\text { Mean of Center } \\
\text { Point Pixel Distances }\end{array}$ & $\begin{array}{c}\text { Mean of Cross } \\
\text { Correlation Btwn } \\
\text { Current and Previous } \\
\text { Frame }\end{array}$ & $\begin{array}{c}\text { Mean of Cross } \\
\text { Correlation Btwn } \\
\text { Current and Model } \\
\text { Frame }\end{array}$ \\
\hline 64.08696 & 7.962489 & 0.890499 & 0.648316 \\
\hline
\end{tabular}


Rotation Test: Outdoor 4, D = 12ft

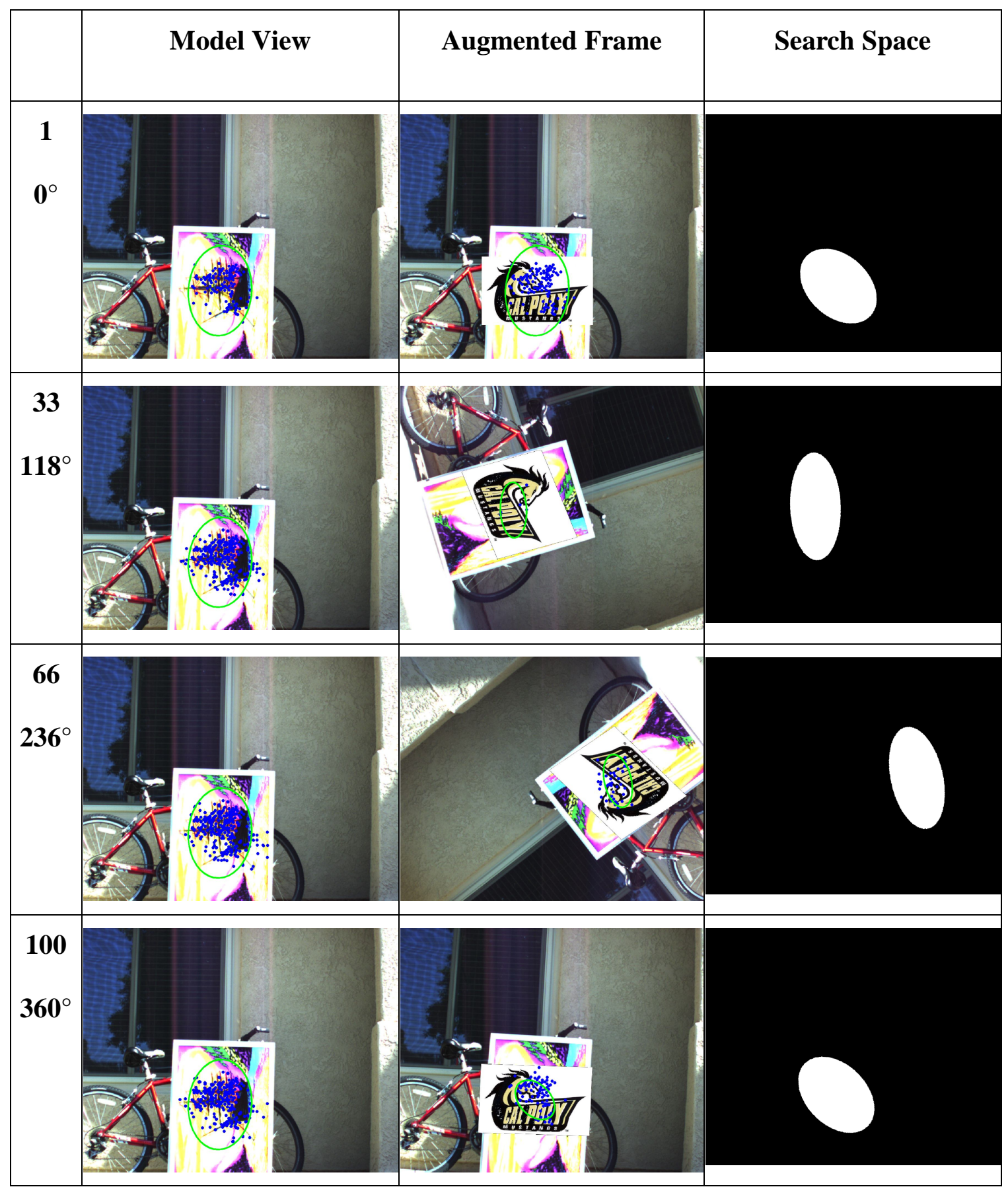

Figure 67: Rotation Images, Outdoor 4 
Start Frame $(1)=0^{\circ}$, End Frame $(100)=360^{\circ}$, Delta $=3.6^{\circ}$ per Frame

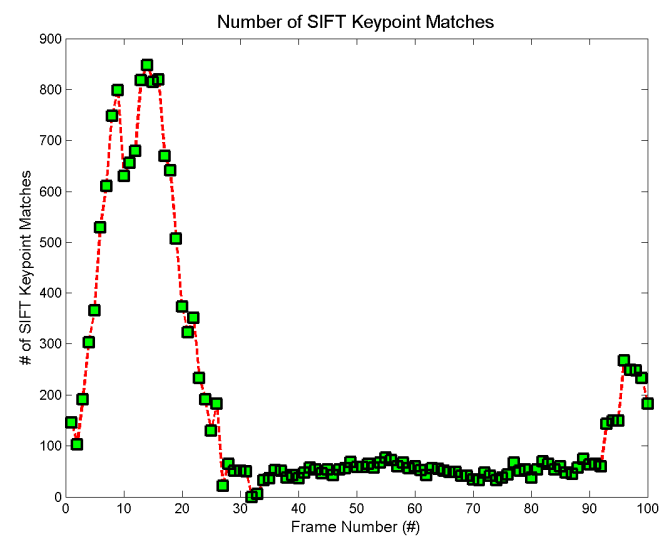

a)

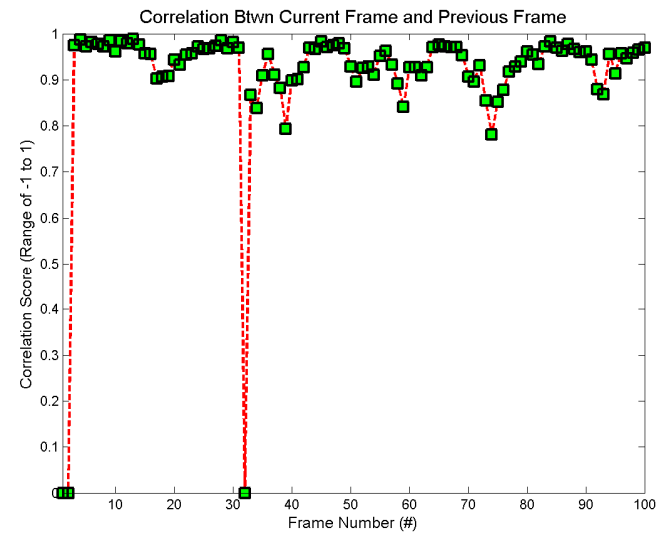

c)

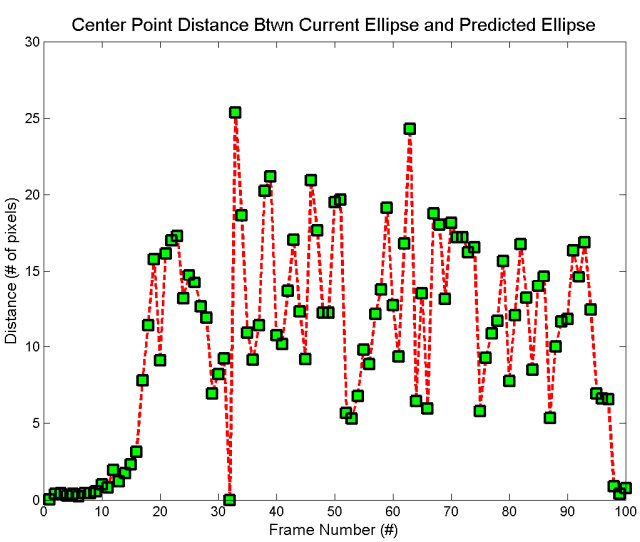

b)

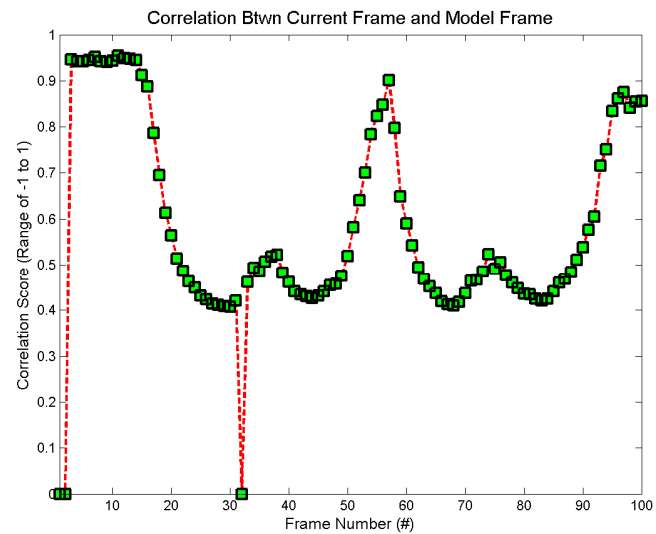

d)

Figure 68: Rotation Plots Outdoor 4, a) SIFT Keypoint Matches, b) Center Point Pixel Distances, c) Cross Correlation Btwn Current and Previous Frame, d) Cross Correlation Btwn Current and Model Frame

Table 31: Rotation Data Outdoor 4

\begin{tabular}{|r|r|r|r|}
\hline $\begin{array}{c}\text { Mean of SIFT } \\
\text { Keypoint Matches }\end{array}$ & $\begin{array}{c}\text { Mean of Center } \\
\text { Point Pixel Distances }\end{array}$ & $\begin{array}{c}\text { Mean of Cross } \\
\text { Correlation Btwn } \\
\text { Current and Previous } \\
\text { Frame }\end{array}$ & $\begin{array}{c}\text { Mean of Cross } \\
\text { Correlation Btwn } \\
\text { Current and Model } \\
\text { Frame }\end{array}$ \\
\hline 176.07 & 10.59885 & 0.911436 & 0.580254 \\
\hline
\end{tabular}




\section{Scale Tests}

Scale Test: Ideal

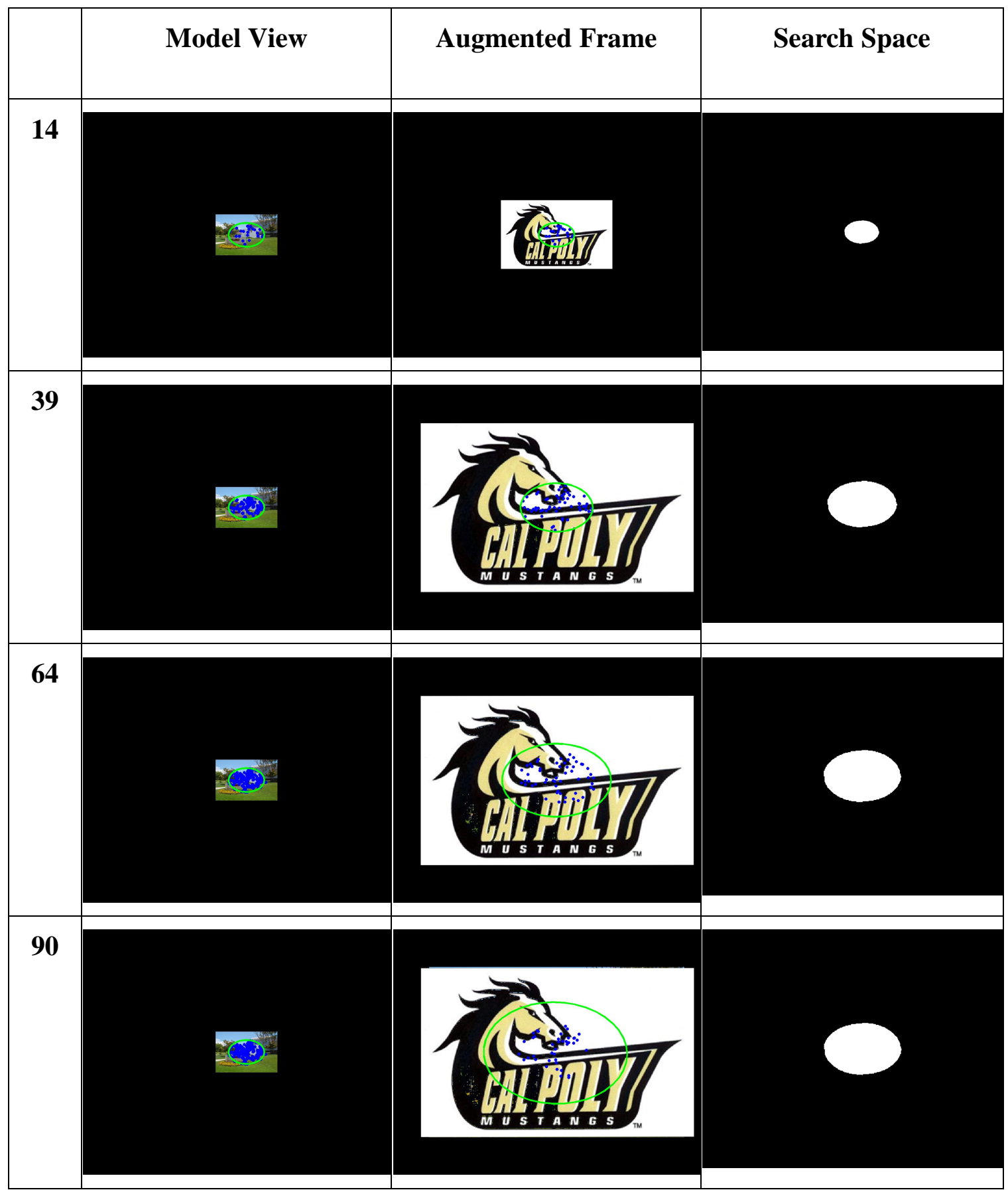

Figure 69: Scale Images, Ideal 


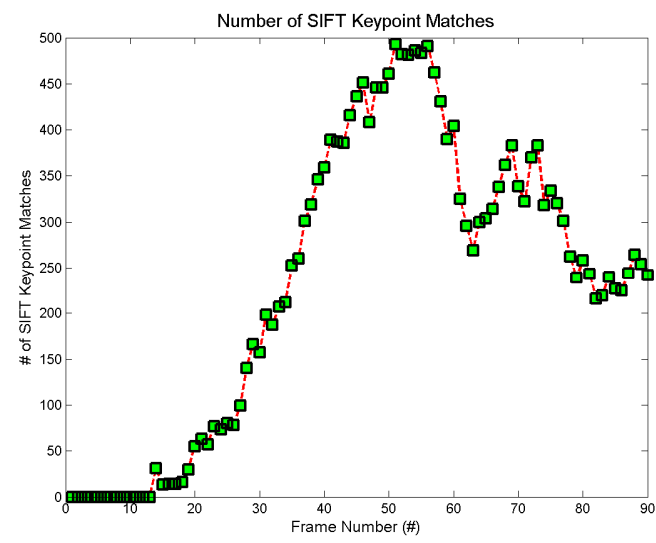

a)

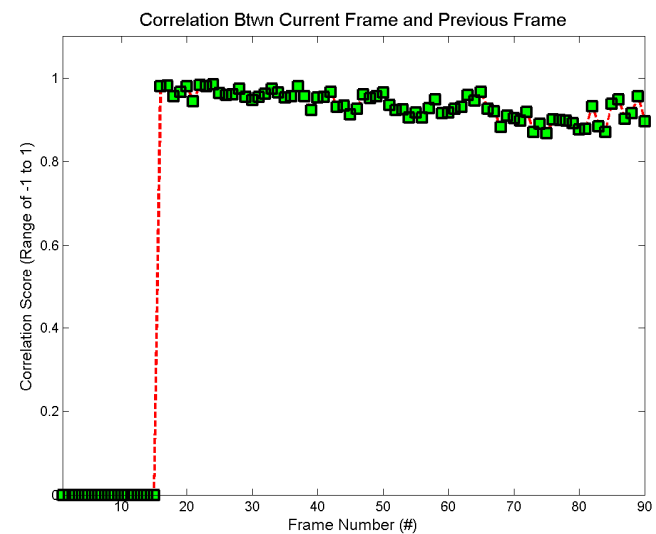

c)

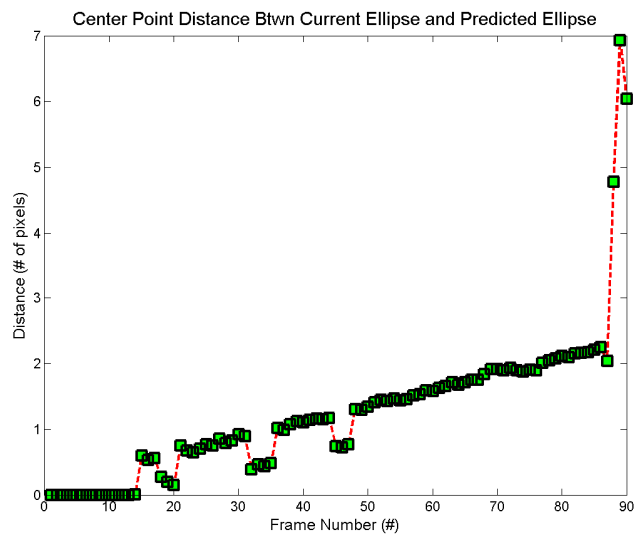

b)

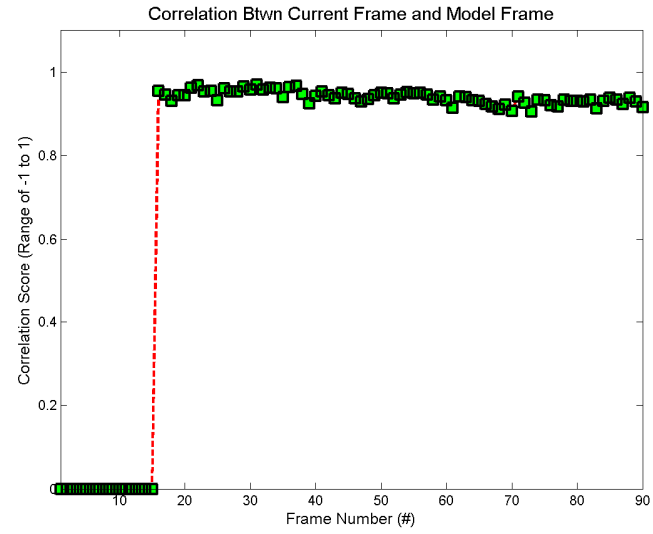

d)

Figure 70: Scale Plots Ideal, a) SIFT Keypoint Matches, b) Center Point Pixel Distances, c) Cross Correlation Btwn Current and Previous Frame, d) Cross Correlation Btwn Current and Model Frame

Table 32: Scale Data Ideal

\begin{tabular}{|r|c|c|c|}
\hline $\begin{array}{c}\text { Mean of SIFT } \\
\text { Keypoint Matches }\end{array}$ & $\begin{array}{c}\text { Mean of Center } \\
\text { Point Pixel } \\
\text { Distances }\end{array}$ & $\begin{array}{c}\text { Mean of Cross } \\
\text { Correlation Btwn } \\
\text { Current and Previous } \\
\text { Frame }\end{array}$ & $\begin{array}{c}\text { Mean of Cross } \\
\text { Correlation Btwn } \\
\text { Current and Model } \\
\text { Frame }\end{array}$ \\
\hline 237.1667 & 1.262379 & 0.779065 & 0.783411 \\
\hline
\end{tabular}


Scale Test: Indoor 2

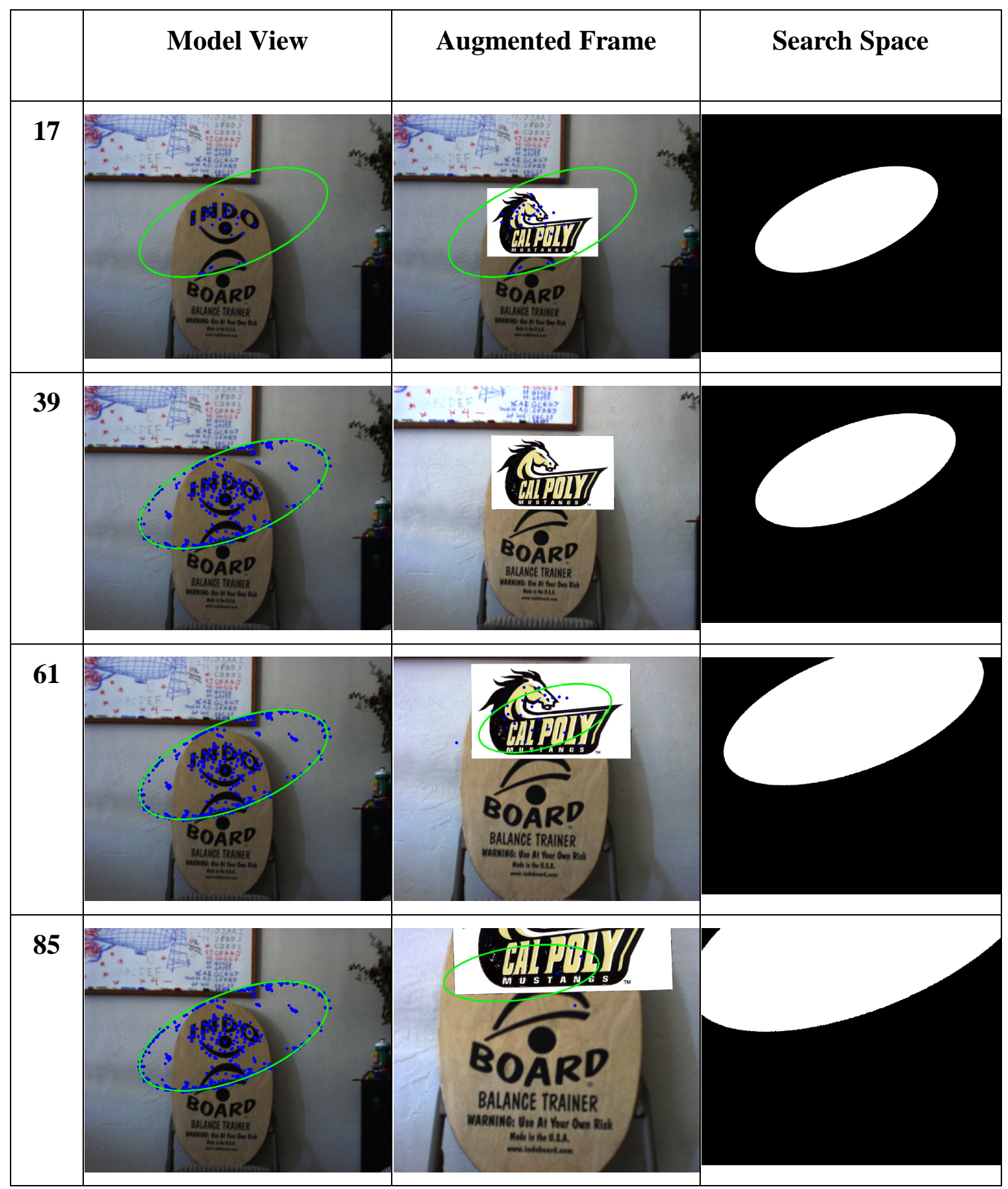

Figure 71: Scale Images, Indoor 2 


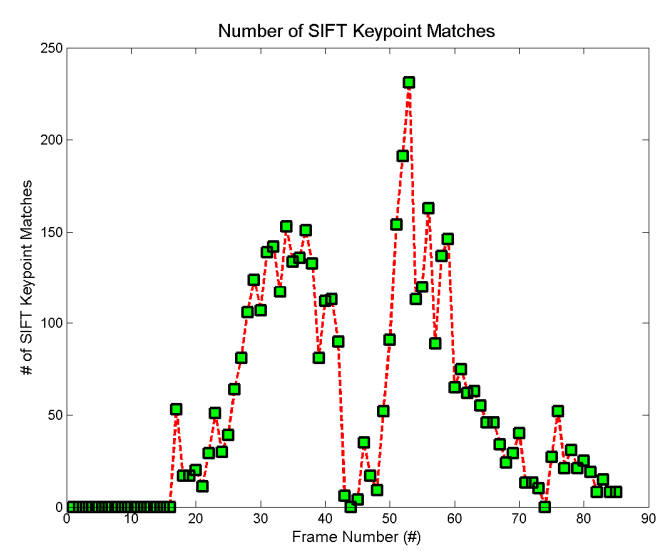

a)

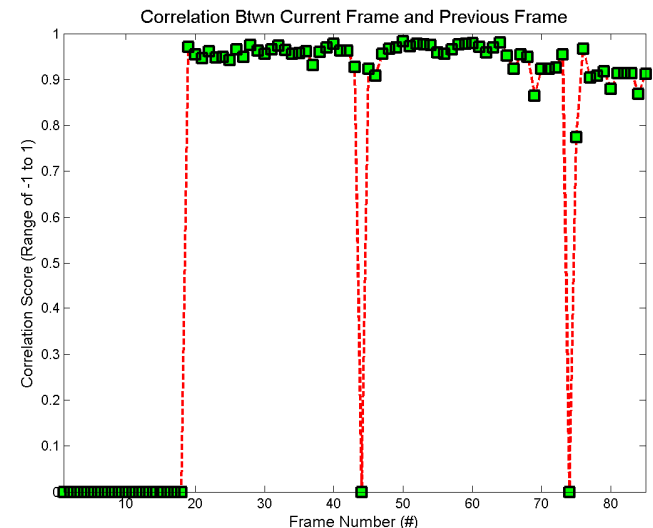

c)

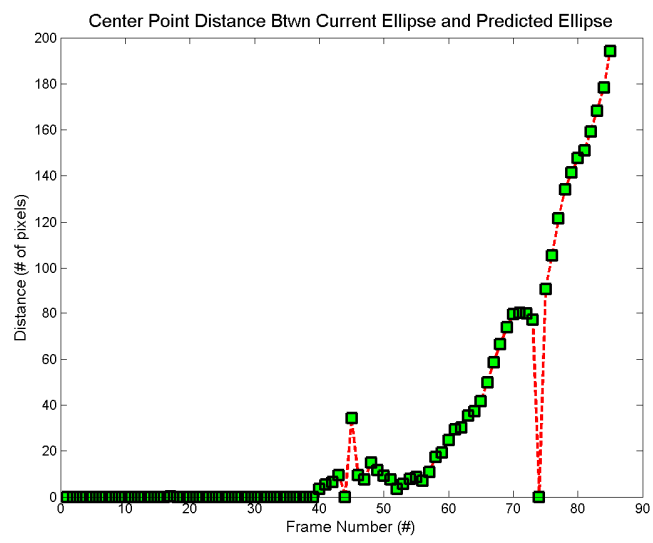

b)

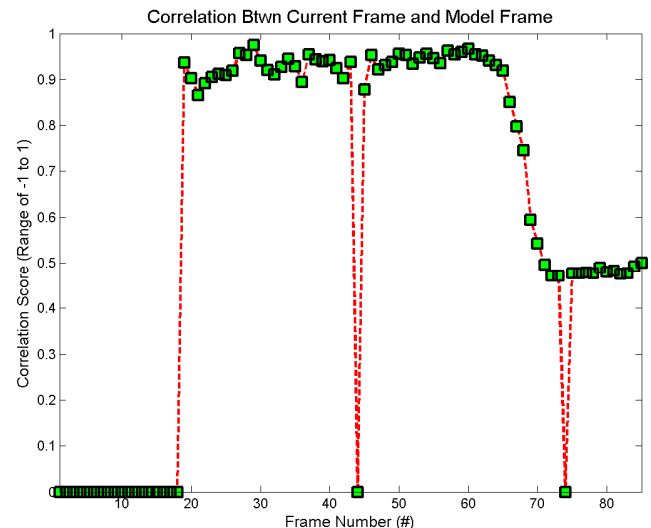

d)

Figure 72: Scale Plots Indoor 2, a) SIFT Keypoint Matches, b) Center Point Pixel Distances, c) Cross Correlation Btwn Current and Previous Frame, d) Cross Correlation Btwn Current and Model Frame

Table 33: Scale Data Indoor 2

\begin{tabular}{|r|r|r|r|}
\hline $\begin{array}{c}\text { Mean of SIFT } \\
\text { Keypoint Matches }\end{array}$ & $\begin{array}{c}\text { Mean of Center } \\
\text { Point Pixel } \\
\text { Distances }\end{array}$ & $\begin{array}{c}\text { Mean of Cross } \\
\text { Correlation Btwn } \\
\text { Current and Previous } \\
\text { Frame }\end{array}$ & $\begin{array}{c}\text { Mean of Cross } \\
\text { Correlation Btwn } \\
\text { Current and Model } \\
\text { Frame }\end{array}$ \\
\hline 54.32941 & 30.04514 & 0.723223 & 0.625814 \\
\hline
\end{tabular}




\section{Scale Test: Outdoor 1}

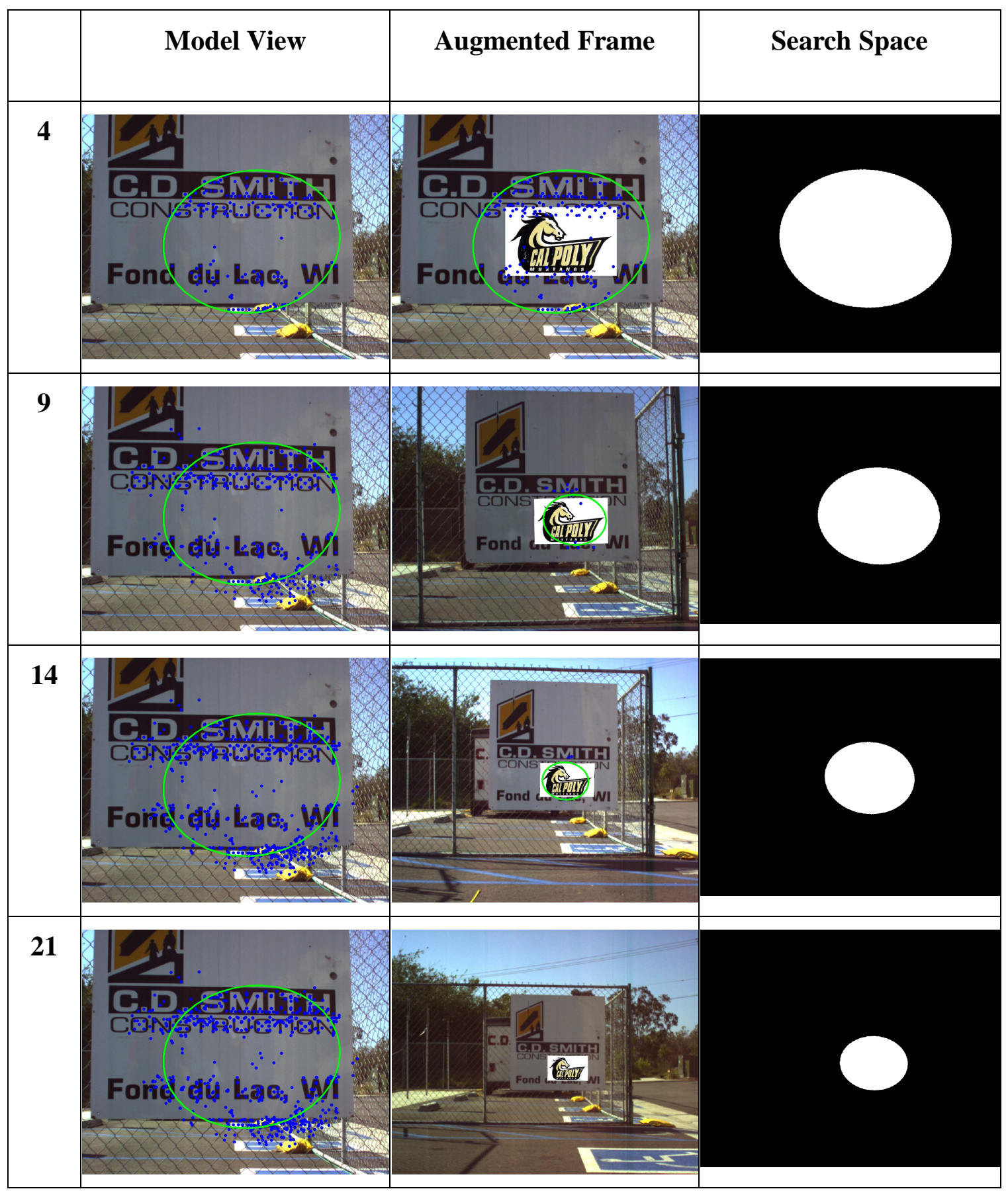

Figure 73: Scale Images, Outdoor 1 


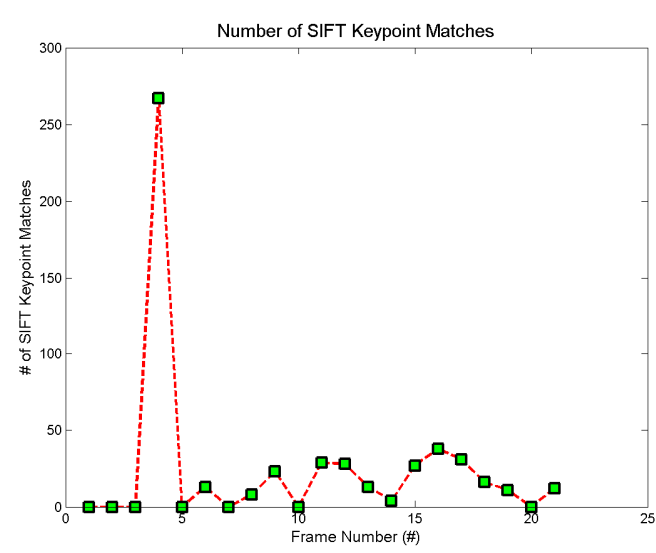

a)

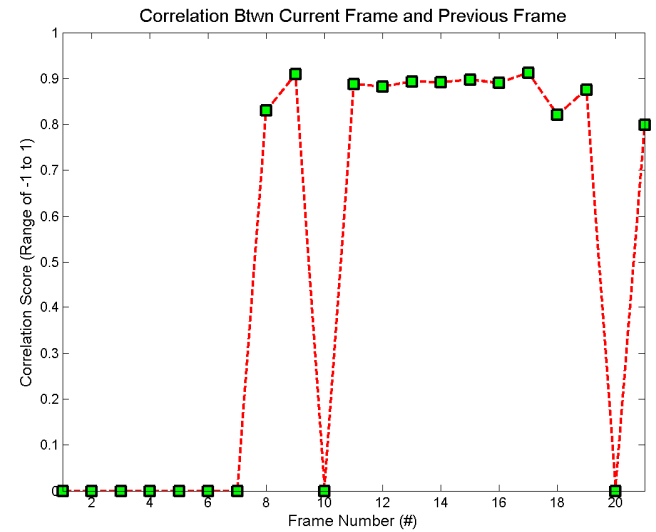

c)

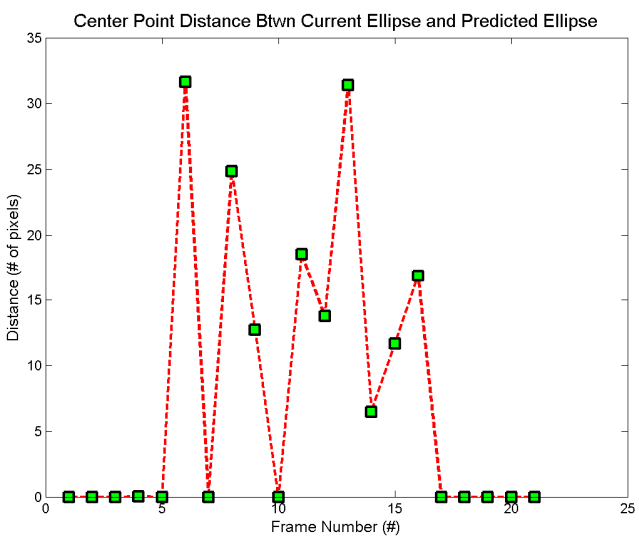

b)

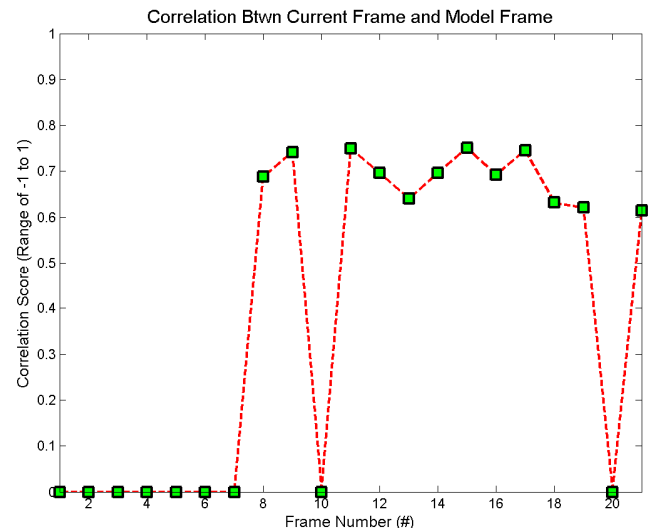

d)

Figure 74: Scale Plots Outdoor 1, a) SIFT Keypoint Matches, b) Center Point Pixel Distances, c) Cross Correlation Btwn Current and Previous Frame, d) Cross Correlation Btwn Current and Model Frame

Table 34: Scale Data Outdoor 1

\begin{tabular}{|r|c|c|c|}
\hline $\begin{array}{c}\text { Mean of SIFT } \\
\text { Keypoint Matches }\end{array}$ & $\begin{array}{c}\text { Mean of Center } \\
\text { Point Pixel } \\
\text { Distances }\end{array}$ & $\begin{array}{c}\text { Mean of Cross } \\
\text { Correlation Btwn } \\
\text { Current and Previous } \\
\text { Frame }\end{array}$ & $\begin{array}{c}\text { Mean of Cross } \\
\text { Correlation Btwn } \\
\text { Current and Model } \\
\text { Frame }\end{array}$ \\
\hline 24.7619 & 7.995469 & 0.49941 & 0.39367 \\
\hline
\end{tabular}




\section{Scale Test: Outdoor 2}

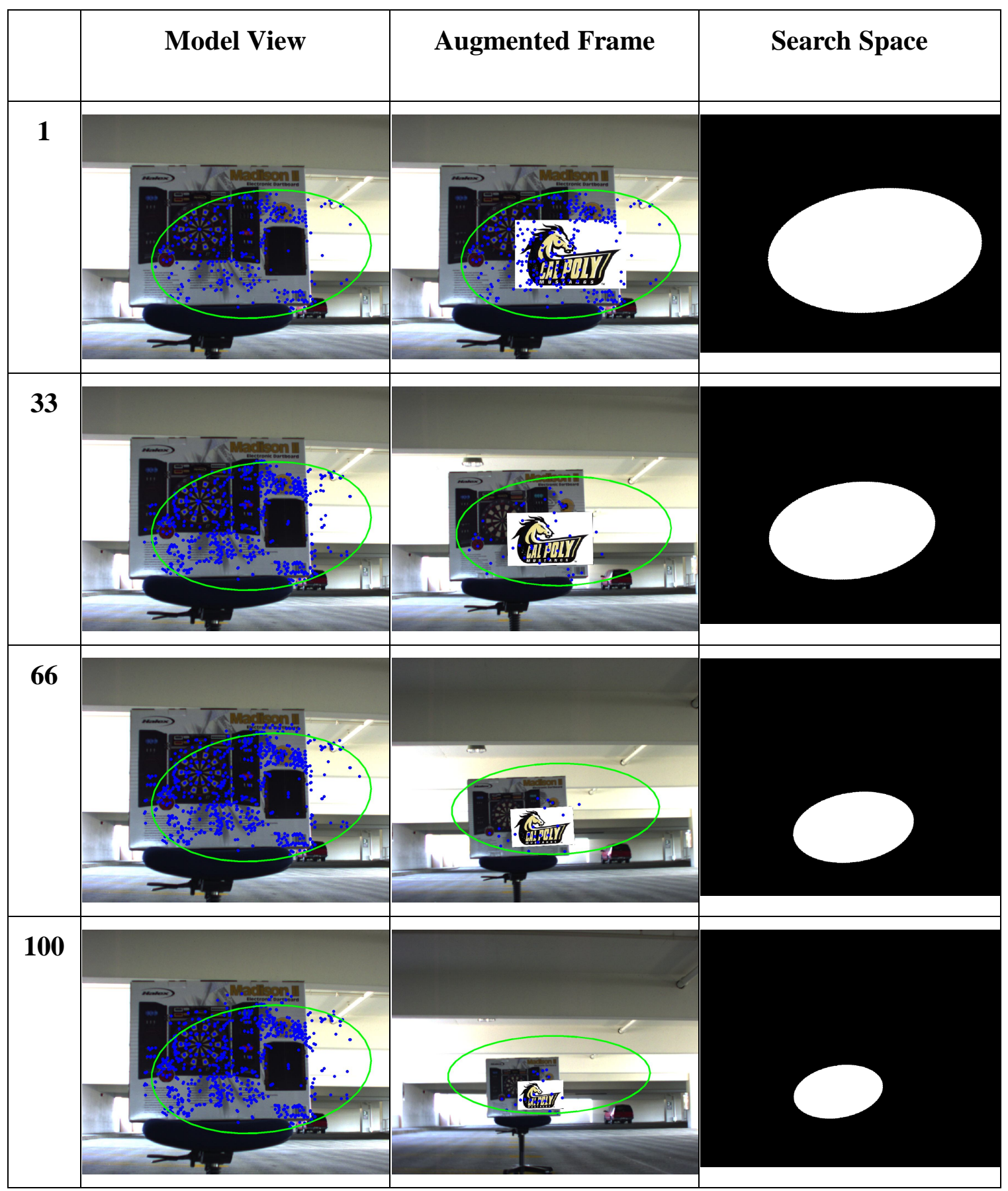

Figure 75: Scale Images, Outdoor 2 


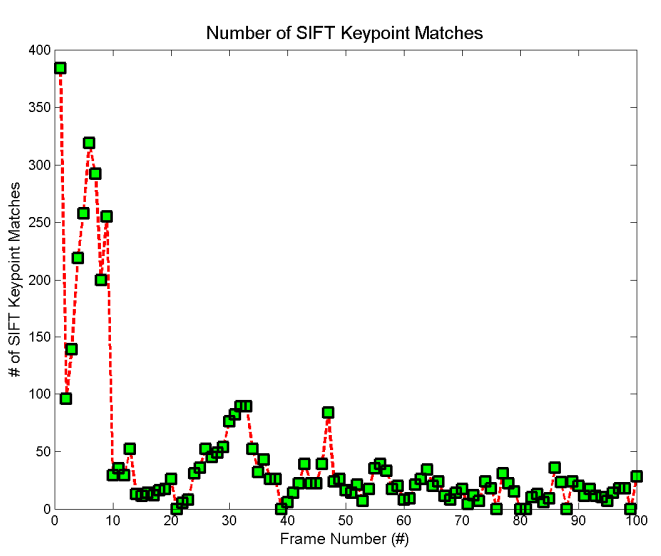

a)

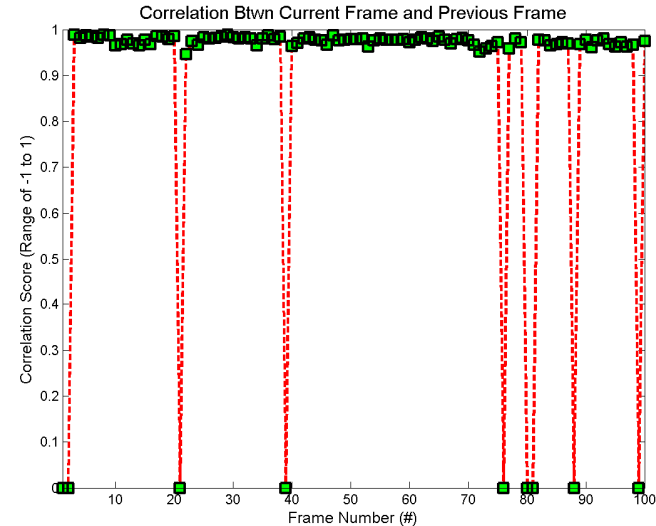

c)

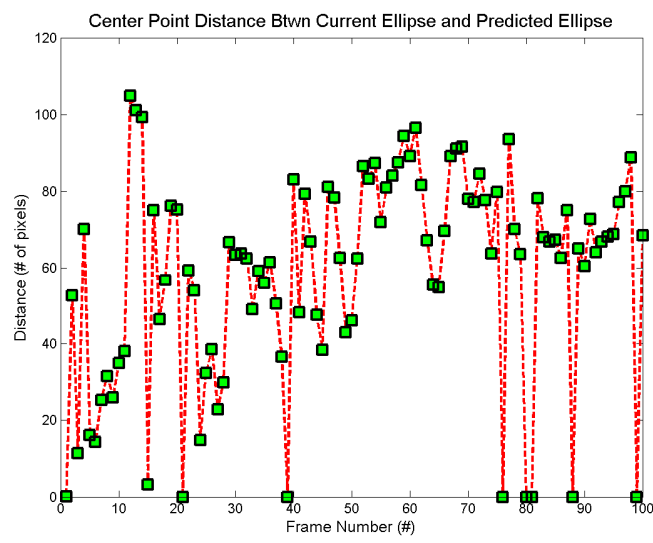

b)

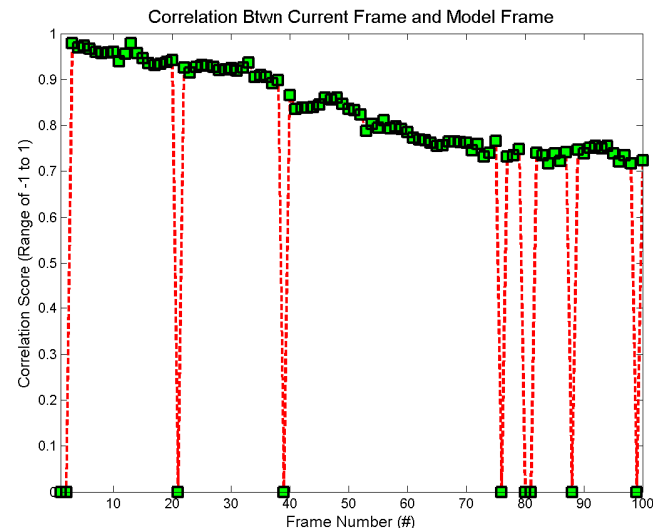

d)

Figure 76: Scale Plots Outdoor 2, a) SIFT Keypoint Matches, b) Center Point Pixel Distances, c) Cross Correlation Btwn Current and Previous Frame, d) Cross Correlation Btwn Current and Model Frame

Table 35: Scale Data Outdoor 2

\begin{tabular}{|r|c|c|c|}
\hline $\begin{array}{c}\text { Mean of SIFT } \\
\text { Keypoint Matches }\end{array}$ & $\begin{array}{c}\text { Mean of Center } \\
\text { Point Pixel } \\
\text { Distances }\end{array}$ & $\begin{array}{c}\text { Mean of Cross } \\
\text { Correlation Btwn } \\
\text { Current and Previous } \\
\text { Frame }\end{array}$ & $\begin{array}{c}\text { Mean of Cross } \\
\text { Correlation Btwn } \\
\text { Current and Model } \\
\text { Frame }\end{array}$ \\
\hline 43.28 & 58.65419 & 0.887533 & 0.762544 \\
\hline
\end{tabular}




\section{Scale Test: Outdoor 3}

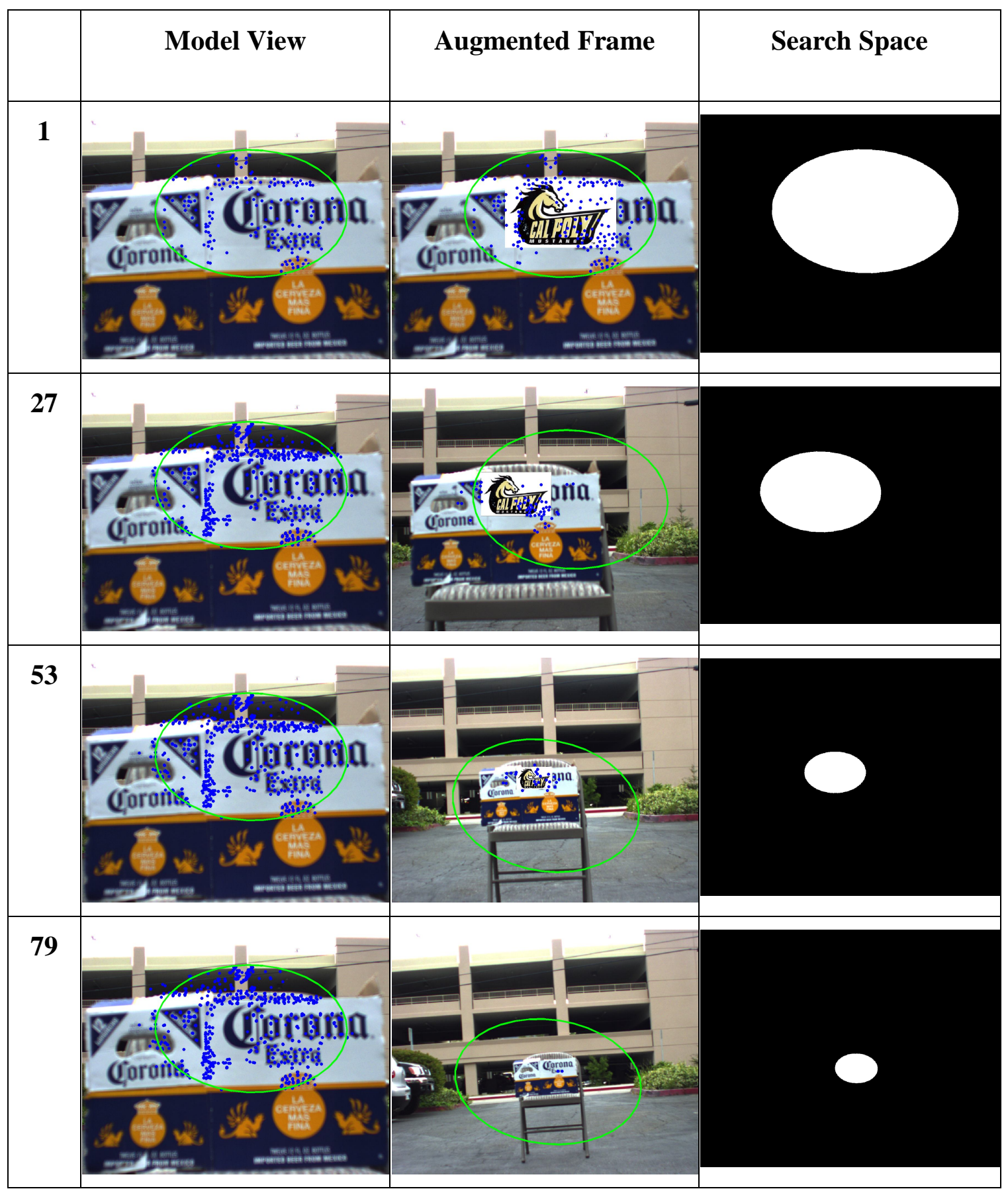

Figure 77: Scale Images, Outdoor 3 


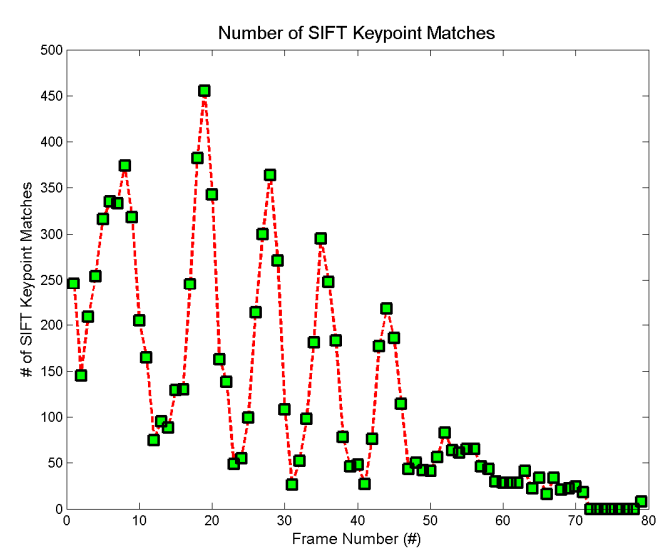

a)

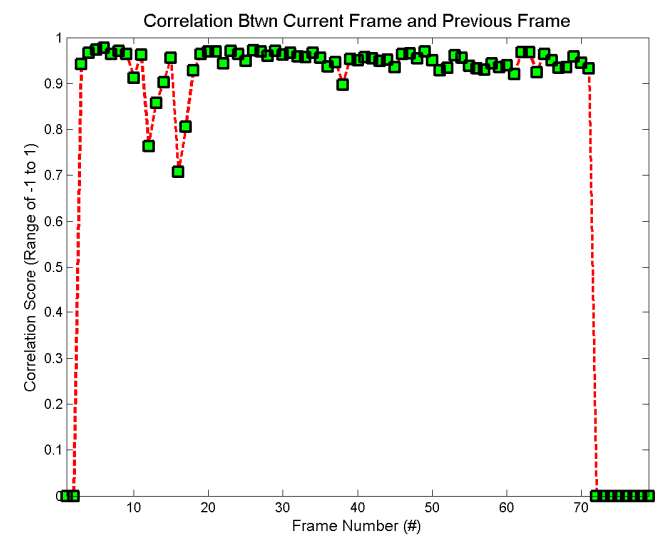

c)

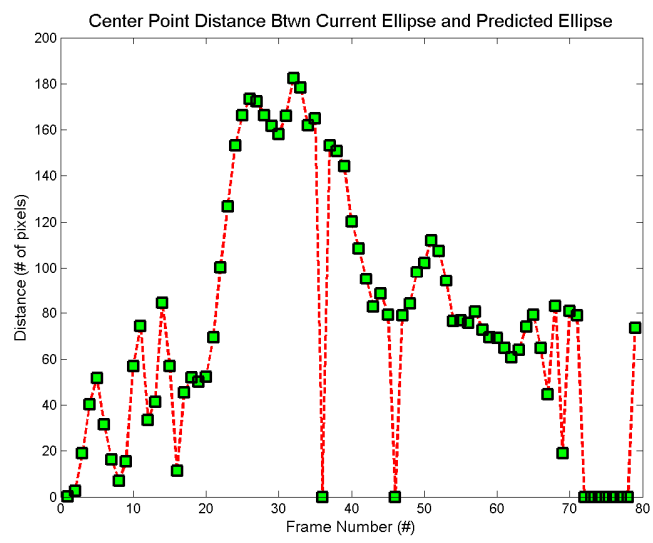

b)

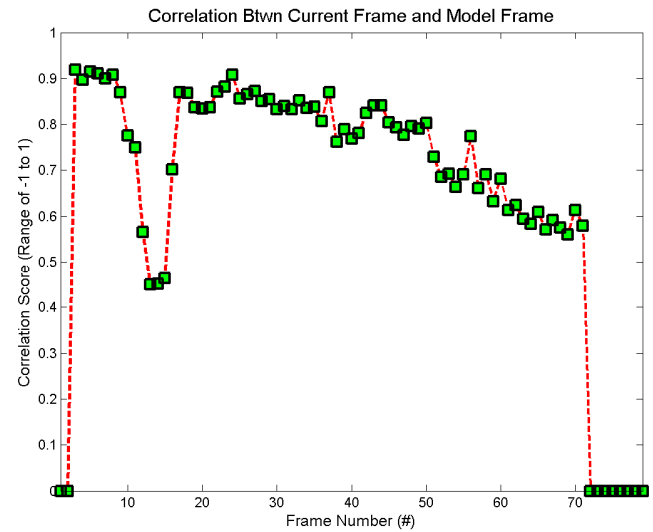

d)

Figure 78: Scale Plots Outdoor 3, a) SIFT Keypoint Matches, b) Center Point Pixel Distances, c) Cross Correlation Btwn Current and Previous Frame, d) Cross Correlation Btwn Current and Model Frame

Table 36: Scale Data Outdoor 3

\begin{tabular}{|r|c|c|c|}
\hline $\begin{array}{c}\text { Mean of SIFT } \\
\text { Keypoint Matches }\end{array}$ & $\begin{array}{c}\text { Mean of Center } \\
\text { Point Pixel } \\
\text { Distances }\end{array}$ & $\begin{array}{c}\text { Mean of Cross } \\
\text { Correlation Btwn } \\
\text { Current and Previous } \\
\text { Frame }\end{array}$ & $\begin{array}{c}\text { Mean of Cross } \\
\text { Correlation Btwn } \\
\text { Current and Model } \\
\text { Frame }\end{array}$ \\
\hline 122.3924 & 76.58836 & 0.822148 & 0.660416 \\
\hline
\end{tabular}


Scale Test: Outdoor 4

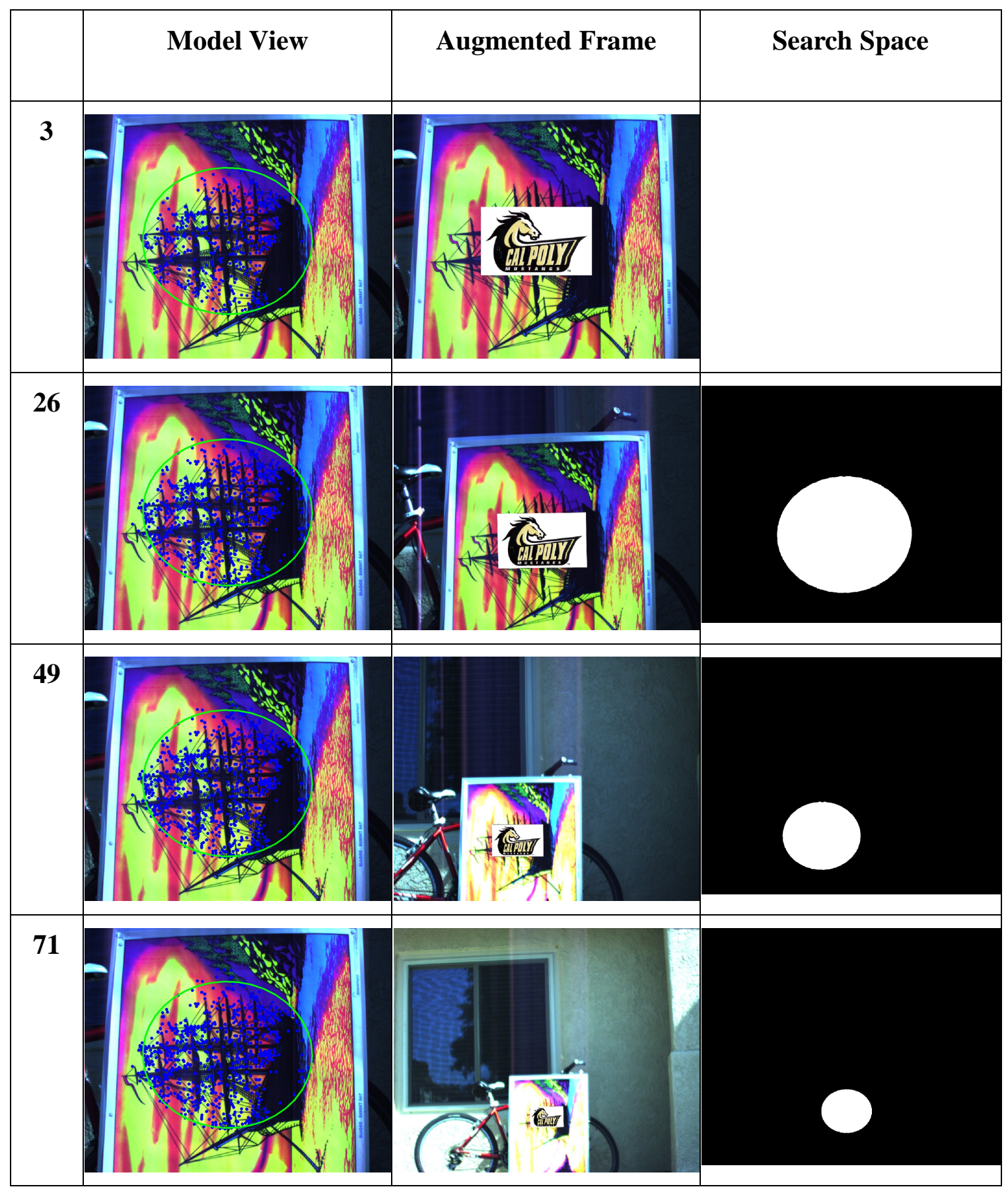

Figure 79: Scale Images, Outdoor 4 


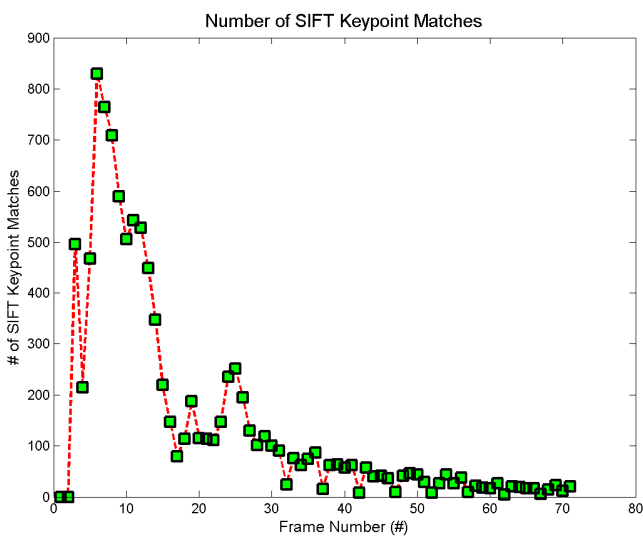

a)

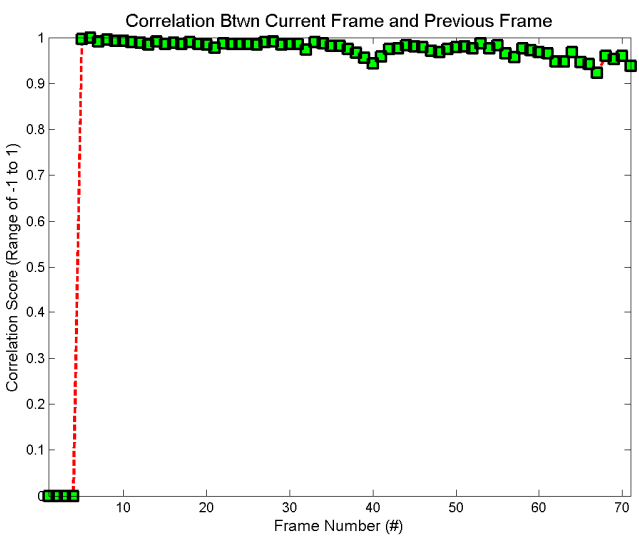

c)

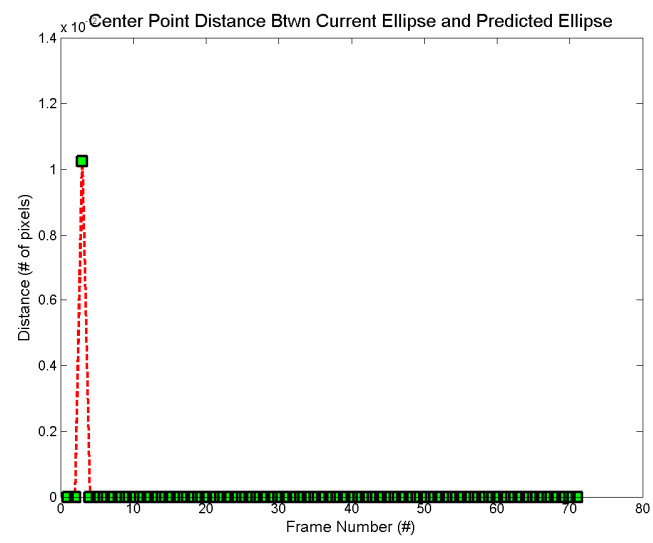

b)

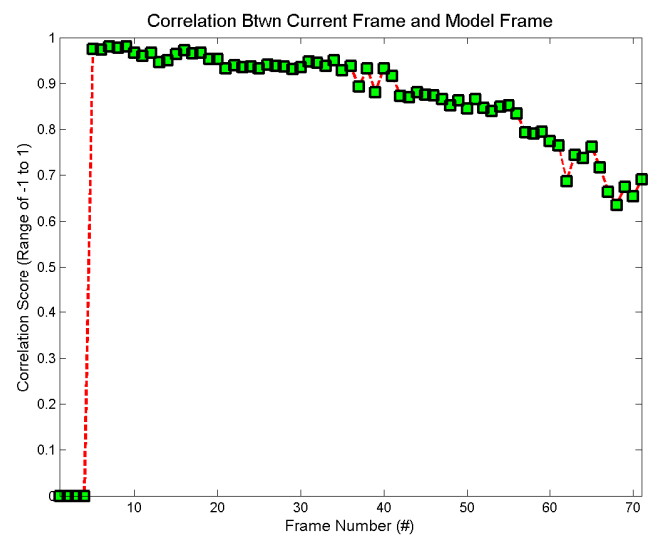

d)

Figure 80: Scale Plots Outdoor 4, a) SIFT Keypoint Matches, b) Center Point Pixel Distances, c) Cross Correlation Btwn Current and Previous Frame, d) Cross Correlation Btwn Current and Model Frame

Table 37: Scale Data Outdoor 4

\begin{tabular}{|r|r|r|r|}
\hline $\begin{array}{c}\text { Mean of SIFT } \\
\text { Keypoint Matches }\end{array}$ & $\begin{array}{c}\text { Mean of Center Point } \\
\text { Pixel Distances }\end{array}$ & $\begin{array}{c}\text { Mean of Cross } \\
\text { Correlation Btwn } \\
\text { Current and Previous } \\
\text { Frame }\end{array}$ & $\begin{array}{c}\text { Mean of Cross } \\
\text { Correlation Btwn } \\
\text { Current and Model } \\
\text { Frame }\end{array}$ \\
\hline 143.8451 & 0 & 0.920767 & 0.82782 \\
\hline
\end{tabular}




\section{List of References}

[1] Azuma, R. T. "A Survey of Augmented Reality," Presence: Teleoperators and Virtual Environments, Vol. 6, No. 4, Aug. 1997, pp. 355-385.

[2] Shahzad, M., Roth, G., McDonald, C., "Robust Corner Tracking for Real-time Augmented Reality," Proceedings of Vision Interface, 2002.

[3] Kanbara, M., Takemuraa, H., Yokoya, N., and Iwasa, H., "A Stereo Vision Based Augmented Reality System with a Wide Range of Registration." IEEE International Conference on Pattern Recognition, Sept. 2000.

[4] Lee, S., Jang, D., Kim, E., Hong, S., and Han, J., "Stereo Vision Based Realtime Workspace Modeling for Robotic Manipulation," IEEE/RSJ International Conference on Intelligent Robots and Systems, 2006.

[5] Moradi, H., Choi, J., Kim, E., Lee, S., "A Real-Time Wall Detection Method for Indoor Environments," IEEE/RSJ International Conference on Intelligent Robots and Systems, Oct. 2006, pp. 4551-4557.

[6] Mikolajczyk, K., Tuytelaars, T., Schmid, C., Zisserman, A., Matas, J., Schaffalitzky, F., Kadir, T., and Gool, L. V., "A Comparison of Affine Region Detectors." International Journal of Computer Vision, Vol. 65, 2005, pp. 43-72.

[7] Tuytelaars, T., and Van Gool, L., "Content-based image retrieval based on local affinely invariant regions." International Conf. on Visual Information Systems, 1999, pp. 493-500.

[8] Tuytelaars, T., and Van Gool, L., "Wide Baseline Stereo Matching Based on Local, Affinely Invariant Regions." Proceedings of the 11th British Machine Vision Conference, 2000, pp. 412-425.

[9] Mikolajczyk, K. and Schmid, C., "An Affine Invariant Interest Point Detector," Proceedings of the 7th European Conference on Computer Vision, 2002.

[10] Harris, C. and Stephens, M., "A Combined Corner and Edge Detector." Alvey Vision Conference, 1988, pp. 147-151.

[11] Matas, J., Chum, O., Urban, M., and Pajdla, T., "Robust Wide-Baseline Stereo from Maximally Stable Extremal Regions," Proceedings of the British Machine Vision Conference, 2002, pp. 384-393.

[12] Mikolajczyk, K., and Schmid, C., "A Performance Evaluation of Local Descriptors," IEEE Computer Society Conference on Computer Vision and Pattern Recognition, 2003.

[13] Lowe., D. G., "Object Recognition from Local Scale-Invariant Features," IEEE International Conference on Computer Vision, 1999, pp. 1150-1157.

[14] Skrypnyk, I., Lowe, D.G., "Scene Modeling, Recognition and Tracking with Invariant Image Features," Proceedings of International Symposium on Mixed and Augmented Reality, 2004, pp. 110-119.

[15] Mikolajczyk, K., "Detection of Local Features Invariant to Affine Transformations," PhD thesis, Institut National Polytechnique de Grenoble, 2002.

[16] Brown, M., and Lowe, D., "Invariant Features from Interest Point Groups," Proceedings of the 13th British Machine Vision Conference, 2002, pp. 253-262. 